\title{
Enantioselective Metal-Free Hydrogenations of Disubstituted Quinolines
}

Zhenhua Zhang and Haifeng Du*

Beijing National Laboratory for Molecular Sciences, CAS Key Laboratory of Molecular Recognition and Function, Institute of Chemistry, Chinese Academy of Sciences, Beijing 100190, China

Supporting Information 
General consideration: All air-sensitive compounds were handled under an atmosphere of argon or in a nitrogen-filled glovebox. ${ }^{1} \mathrm{H}$ NMR, ${ }^{19} \mathrm{~F}$ NMR and ${ }^{13} \mathrm{C}$ NMR spectra were recorded on Bruker AV 400 or 500 at ambient temperature with $\mathrm{CDCl}_{3}$ as solvent and TMS as internal standard. Chemical shifts $(\delta)$ were given in ppm, referenced to the residual proton resonance of TMS (0), to the carbon resonance of the $\mathrm{CDCl}_{3}$ (77.23). Coupling constants $(\mathrm{J})$ were given in $\mathrm{Hertz}(\mathrm{Hz})$. IR spectrums were recorded on Perkin-Elmer-983 spectrometer. Optical rotations were measured with PerkinElmer 341 polarimeter. Flash column chromatography was performed on silica gel (200-300 mesh). All solvents were purified by conventional methods, distilled before use. Commercially available reagents were used without further purification. $\mathrm{H}\left(\mathrm{C}_{6} \mathrm{~F}_{5}\right)_{2}$ was prepared according to reported methods (Parks, D. J.; Piers, W. E. von H.; Yap, G. P. A. Organometallics 1998, 17, 5492). Chiral diene 3b was prepared according to the method previously reported in the literature by our group (Cao, Z.; Du, H. Org. Lett. 2010, 12, 2602).

General Procedure for the Synthesis of 2,4-Disubstituted Quinolines: A mixture of compound 2-aminobenzophenone $(10 \mathrm{mmol})$, acetophenone $(12 \mathrm{mmol})$, and $\mathrm{InCl}_{3}(2 \mathrm{mmol})$ under solvent-free conditions was heated at $100{ }^{\circ} \mathrm{C}$ in a $25 \mathrm{~mL}$ round-bottomed flask. After completion of the reaction (monitored by TLC), water $(15 \mathrm{~mL})$ was added and the reaction mixture was extracted with EtOAc $(3 \times$ $10 \mathrm{~mL}$ ). The combined organic extract was washed with brine and dried over anhydrous $\mathrm{Na}_{2} \mathrm{SO}_{4}$. The solvent was evaporated under reduced pressure and the residue was purified by column chromatography on silica gel (petroleum ether/ethyl acetate $=20 / 1$ ) to afford 2,4-disubstituted quinolines.

Chanda, T.; Verma, R. K.; Singh, M. S. Chem. Asian J. 2012, 7, 778.

General Procedure for the Synthesis of 2,3-Disubstituted Quinolines: To a solution of 2-aminobenzyl alcohol $(10 \mathrm{mmol})$ in 1,4-dioxane $(30 \mathrm{~mL})$ were added the propiophenone $(10 \mathrm{mmol})$, benzophenone $(10 \mathrm{mmol})$, and potassium tertbutoxide $(10 \mathrm{mmol})$ under an argon atmosphere. The resulting mixture was heated at $90{ }^{\circ} \mathrm{C}$ with stirring for $30 \mathrm{~min}$. Then, the reaction was filtered through Celite and cooled to room temperature. The resulting solution was added to a saturated solution of $\mathrm{NH}_{4} \mathrm{Cl}$ $(50 \mathrm{~mL})$ and extracted with acetate $(3 \times 10 \mathrm{~mL})$. The combined organic layers were dried over anhydrous 
$\mathrm{Na}_{2} \mathrm{SO}_{4}$, filtered, and evaporated to give a residue, which was purified either by column chromatography on silica gel to afford 2,3-disubstituted quinolines.

Martínez, R.; Ramón, J. D.; Yus, M. J. Org. Chem. 2008, 73, 9778.

Representative procedure for the metal-free catalytic asymmetric hydrogenation of 2,4-disubstituted quinolines (5a) (Table 2, entry 1): To a glass test tube (10 mL) was added $\mathrm{HB}\left(\mathrm{C}_{6} \mathrm{~F}_{5}\right)_{2}$ $(0.0138 \mathrm{~g}, 0.04 \mathrm{mmol})$, chiral diene $3 \mathbf{b}(0.0126 \mathrm{~g}, 0.02 \mathrm{mmol})$ and dry toluene $(0.6 \mathrm{~mL})$ in a nitrogen atmosphere glovebox. The resulting mixture was stirred for $10 \mathrm{~min}$ at room temperature followed by addition of 2,4-disubstituted quinoline $4 \mathbf{a}(0.1124 \mathrm{~g}, 0.4 \mathrm{mmol})$ and dry toluene $(0.2 \mathrm{~mL})$. The tube was then moved to a stainless-steel autoclave. After being sealed, the autoclave was purged three times with $\mathrm{H}_{2}$ and the final pressure of hydrogen was adjusted to 20 bar. The reaction mixture was stirred at $15^{\circ} \mathrm{C}$ for $24 \mathrm{~h}$, and the solvent was removed under reduced pressure. The crude residue was purified by flash chromatography on silica gel (petroleum ether/ethyl acetate $=20 / 1$ ) to give the desired chiral cis-5a as a white solid $(0.1037 \mathrm{~g}, 91 \%$ yield, $91 \%$ ee).

Representative procedure for the metal-free catalytic asymmetric hydrogenation of 2,3-disubstituted quinolines (7a) (Table 3, entry 1): To a glass test tube $(10 \mathrm{~mL})$ was added $\mathrm{HB}\left(\mathrm{C}_{6} \mathrm{~F}_{5}\right)_{2}$ $(0.0138 \mathrm{~g}, 0.04 \mathrm{mmol})$, chiral diene $3 \mathbf{b}(0.0126 \mathrm{~g}, 0.02 \mathrm{mmol})$ and dry toluene $(3.0 \mathrm{~mL})$ in a nitrogen atmosphere glovebox. The resulting mixture was stirred for $10 \mathrm{~min}$ at room temperature followed by addition of 2,3-disubstituted quinoline $6 \mathbf{a}(0.0876 \mathrm{~g}, 0.4 \mathrm{mmol})$ and dry toluene $(1.0 \mathrm{~mL})$. The tube was then moved to a stainless-steel autoclave. After being sealed, the autoclave was purged three times with $\mathrm{H}_{2}$ and the final pressure of hydrogen was adjusted to 20 bar. The reaction mixture was stirred at $0{ }^{\circ} \mathrm{C}$ for $24 \mathrm{~h}$, and the solvent was removed under reduced pressure. The crude residue was purified by flash chromatography on silica gel (petroleum ether/ethyl acetate $=20 / 1$ ) to give compound cis-7a as a yellow oil (0.0812 g, $91 \%$ yield, $69 \%$ ee). 
<smiles>c1ccc(-c2cc(-c3ccccc3)c3ccccc3n2)cc1</smiles>

2,4-diphenylquinoline (4a): $2.28 \mathrm{~g}\left(81 \%\right.$ yield), ${ }^{1} \mathrm{H} \mathrm{NMR}\left(400 \mathrm{MHz}, \mathrm{CDCl}_{3}, \mathrm{ppm}\right) \delta 8.24(\mathrm{~d}, J=8.4 \mathrm{~Hz}$, $1 \mathrm{H}), 8.19(\mathrm{~d}, J=7.6 \mathrm{~Hz}, 2 \mathrm{H}), 7.89(\mathrm{~d}, J=8.4 \mathrm{~Hz}, 1 \mathrm{H}), 7.80(\mathrm{~s}, 1 \mathrm{H}), 7.71(\mathrm{dd}, J=7.6,7.6 \mathrm{~Hz}, 1 \mathrm{H})$, 7.55-7.49 (m, 7H), 7.47-7.43 (m, 2H); ${ }^{13} \mathrm{C}$ NMR (100 MHz, $\left.\mathrm{CDCl}_{3}, \mathrm{ppm}\right) \delta 157.1,149.3,149.0,139.8$, $138.6,130.3,129.74,129.70,129.5,129.0,128.8,128.6,127.8,126.5,125.9,125.8,119.5$.

Chanda, T.; Verma, R. K.; Singh, M. S. Chem. Asian J. 2012, 7, 778<smiles>Clc1ccccc1-c1cc(-c2ccccc2)c2ccccc2n1</smiles>

2-(2-chlorophenyl)-4-phenylquinoline (4b): 1.23 g (39\% yield), White solid, m.p. 91-93 ${ }^{\circ} \mathrm{C}$; IR (film): 1589, 1548, 1491, $1044 \mathrm{~cm}^{-1} ;{ }^{1} \mathrm{H}$ NMR $\left(400 \mathrm{MHz}, \mathrm{CDCl}_{3}, \mathrm{ppm}\right) \delta 8.25(\mathrm{~d}, J=8.4 \mathrm{~Hz}, 1 \mathrm{H}), 7.97(\mathrm{~d}, J=$ 8.4 Hz, 1H), 7.76-7.72 (m, 2H), $7.70(\mathrm{~s}, 1 \mathrm{H}), 7.57-7.54(\mathrm{~m}, 3 \mathrm{H})$, 7.52-7.46 (m, 4H), 7.42-7.34 (m, 2H); ${ }^{13} \mathrm{C} \mathrm{NMR}\left(100 \mathrm{MHz}, \mathrm{CDCl}_{3}, \mathrm{ppm}\right) \delta 157.1,148.8,148.2,139.8,138.2,132.6,131.9,130.3,130.1,129.8$, 129.7, 128.8, 128.6, 127.4, 127.0, 125.9, 125.8, 123.2; HRMS (ESI) calcd for $\mathrm{C}_{21} \mathrm{H}_{15} \mathrm{NCl}(\mathrm{M}+\mathrm{H})$ : 316.0888, Found: 316.0885 .<smiles>Brc1ccccc1-c1cc(-c2ccccc2)c2ccccc2n1</smiles>

2-(2-bromophenyl)-4-phenylquinoline (4c): $1.10 \mathrm{~g}$ (32\% yield), ${ }^{1} \mathrm{H} \mathrm{NMR}\left(400 \mathrm{MHz}, \mathrm{CDCl}_{3}, \mathrm{ppm}\right) \delta$ $8.25(\mathrm{~d}, J=8.4 \mathrm{~Hz}, 1 \mathrm{H}), 7.98(\mathrm{~d}, J=8.4 \mathrm{~Hz}, 1 \mathrm{H}), 7.74(\mathrm{ddd}, J=7.6,7.6,1.2 \mathrm{~Hz}, 1 \mathrm{H}), 7.70(\mathrm{~d}, J=8.0 \mathrm{~Hz}$, 2H), $7.67(\mathrm{~s}, 1 \mathrm{H}), 7.59-7.54(\mathrm{~m}, 3 \mathrm{H}), 7.53-7.48(\mathrm{~m}, 3 \mathrm{H}), 7.45(\mathrm{ddd}, J=7.6,7.6,0.8 \mathrm{~Hz}, 1 \mathrm{H}), 7.29$ (ddd, $J$ $=7.6,7.6,1.6 \mathrm{~Hz}, 1 \mathrm{H}) ;{ }^{13} \mathrm{C} \mathrm{NMR}\left(100 \mathrm{MHz}, \mathrm{CDCl}_{3}, \mathrm{ppm}\right) \delta 158.4,148.7,148.1,141.8,138.2,133.5$, $131.8,130.3,130.2,129.8,129.7,128.8,128.6,127.9,127.0,125.9,125.8,123.2,122.1$. 
Kulkarni, A.; Török, B. Green Chem. 2010, 12, 875.<smiles>Clc1cccc(-c2cc(-c3ccccc3)c3ccccc3n2)c1</smiles>

2-(3-chlorophenyl)-4-phenylquinoline (4d): 1.83 g (58\% yield), White solid, m.p. $94-97{ }^{\circ} \mathrm{C}$; IR (film): 1589, 1574, 1545, $1508 \mathrm{~cm}^{-1} ;{ }^{1} \mathrm{H}$ NMR (400 MHz, $\left.\mathrm{CDCl}_{3}, \mathrm{ppm}\right) \delta$ 8.23-8.21 (m, 2H), 8.05-8.03 (m, 1H), $7.89(\mathrm{~d}, J=8.4 \mathrm{~Hz}, 1 \mathrm{H}), 7.75(\mathrm{~s}, 1 \mathrm{H}), 7.74-7.70(\mathrm{~m}, 1 \mathrm{H}), 7.73-7.44(\mathrm{~m}, 6 \mathrm{H}), 7.41-7.39(\mathrm{~m}, 2 \mathrm{H}) ;{ }^{13} \mathrm{C} \mathrm{NMR}$ $\left(100 \mathrm{MHz}, \mathrm{CDCl}_{3}, \mathrm{ppm}\right) \delta 155.4,149.6,148.9,141.6,138.4,135.1,130.4,130.2,129.9,129.7,129.5$, 128.8, 128.7, 127.9, 126.9, 126.1, 125.9, 125.8, 119.2; HRMS (ESI) calcd for $\mathrm{C}_{21} \mathrm{H}_{15} \mathrm{NCl}(\mathrm{M}+\mathrm{H})$ : 316.0888, Found: 316.0884.<smiles>Brc1cccc(-c2cc(-c3ccccc3)c3ccccc3n2)c1</smiles>

2-(3-bromophenyl)-4-phenylquinoline (4e): $2.62 \mathrm{~g}$ (73\% yield), ${ }^{1} \mathrm{H} \mathrm{NMR}\left(400 \mathrm{MHz}, \mathrm{CDCl}_{3}, \mathrm{ppm}\right) \delta$ $8.37(\mathrm{~s}, 1 \mathrm{H}), 8.22(\mathrm{~d}, J=8.4 \mathrm{~Hz}, 1 \mathrm{H}), 8.08(\mathrm{~d}, J=8.0 \mathrm{~Hz}, 1 \mathrm{H}), 7.89(\mathrm{~d}, J=8.4 \mathrm{~Hz}, 1 \mathrm{H}), 7.74(\mathrm{~s}, 1 \mathrm{H})$, 7.74-7.70 (m, 1H), 7.57-7.44 (m, 7H), $7.35(\mathrm{dd}, J=7.6,7.6 \mathrm{~Hz}, 1 \mathrm{H}) ;{ }^{13} \mathrm{C} \mathrm{NMR}\left(100 \mathrm{MHz}, \mathrm{CDCl}_{3}, \mathrm{ppm}\right)$ $\delta 155.3,149.6,148.9,141.8,138.3,132.4,130.8,130.5,130.3,129.9,129.7,128.8,128.7,126.9,126.2$, $126.1,125.8,123.3,119.1$.

Xiao, F.; Chen, Y.; Liu, Yu.; Wang, J. Tetrahedron 2008, 64, 2755.<smiles>Cc1cccc(-c2cc(-c3ccccc3)c3ccccc3n2)c1</smiles>

4-phenyl-2-(m-tolyl)quinoline (4f): 2.32 g (78\% yield), White solid, m.p. $62-64{ }^{\circ} \mathrm{C}$; IR (film): 1591, 1574, 1548, $1491 \mathrm{~cm}^{-1} ;{ }^{1} \mathrm{H}$ NMR (400 MHz, $\left.\mathrm{CDCl}_{3}, \mathrm{ppm}\right) \delta 8.25(\mathrm{~d}, J=8.4 \mathrm{~Hz}, 1 \mathrm{H}), 8.04(\mathrm{~s}, 1 \mathrm{H}), 7.96(\mathrm{~d}$, 
$J=7.6 \mathrm{~Hz}, 1 \mathrm{H}), 7.90(\mathrm{~d}, J=8.4 \mathrm{~Hz}, 1 \mathrm{H}), 7.81(\mathrm{~s}, 1 \mathrm{H}), 7.73(\mathrm{dd}, J=7.6,7.6 \mathrm{~Hz}, 1 \mathrm{H}), 7.56-7.45(\mathrm{~m}, 6 \mathrm{H})$, $7.41(\mathrm{dd}, J=7.6,7.6 \mathrm{~Hz}, 1 \mathrm{H}), 7.28(\mathrm{~d}, J=7.6 \mathrm{~Hz}, 1 \mathrm{H}), 2.48(\mathrm{~s}, 3 \mathrm{H}) ;{ }^{13} \mathrm{C} \mathrm{NMR}\left(100 \mathrm{MHz}, \mathrm{CDCl}_{3}, \mathrm{ppm}\right) \delta$ $157.3,149.3,149.0,139.8,138.71,138.66,130.34,130.31,129.8,129.7,128.9,128.8,128.6,128.5$, 126.5, 126.0, 125.8, 124.9, 119.7, 21.8; HRMS (ESI) calcd for $\mathrm{C}_{22} \mathrm{H}_{18} \mathrm{~N}(\mathrm{M}+\mathrm{H})$ : 296.1434, Found: 296.1431.<smiles>COc1cccc(-c2cc(-c3ccccc3)c3ccccc3n2)c1</smiles>

2-(3-methoxyphenyl)-4-phenylquinoline (4g): $2.54 \mathrm{~g}$ (81\% yield), ${ }^{1} \mathrm{H} \mathrm{NMR}\left(400 \mathrm{MHz}, \mathrm{CDCl}_{3}, \mathrm{ppm}\right) \delta$ $8.25(\mathrm{~d}, J=8.4 \mathrm{~Hz}, 1 \mathrm{H}), 7.90(\mathrm{~d}, J=8.4 \mathrm{~Hz}, 1 \mathrm{H}), 7.80(\mathrm{~s}, 1 \mathrm{H}), 7.80(\mathrm{~s}, 1 \mathrm{H}), 7.74-7.71(\mathrm{~m}, 2 \mathrm{H}), 7.55-7.40$ $(\mathrm{m}, 7 \mathrm{H}), 7.01(\mathrm{~d}, J=6.8 \mathrm{~Hz}, 1 \mathrm{H}), 3.92(\mathrm{~s}, 3 \mathrm{H}) ;{ }^{13} \mathrm{C} \mathrm{NMR}\left(100 \mathrm{MHz}, \mathrm{CDCl}_{3}, \mathrm{ppm}\right) \delta 160.3,156.9,149.3$, $149.0,141.3,138.6,130.3,130.0,129.8,129.7,128.8,128.6,126.6,126.1,125.8,120.2,119.6,115.7$, 112.9, 55.6.

Xiao, F.; Chen, Y.; Liu, Yu.; Wang, J. Tetrahedron 2008, 64, 2755.<smiles>Clc1ccc(-c2cc(-c3ccccc3)c3ccccc3n2)cc1</smiles>

2-(4-chlorophenyl)-4-phenylquinoline (4h): $2.29 \mathrm{~g}$ (73\% yield), ${ }^{1} \mathrm{H} \mathrm{NMR}\left(400 \mathrm{MHz}, \mathrm{CDCl}_{3}, \mathrm{ppm}\right) \delta$ $8.21(\mathrm{~d}, J=8.4 \mathrm{~Hz}, 1 \mathrm{H}), 8.13(\mathrm{~d}, J=8.4 \mathrm{~Hz}, 2 \mathrm{H}), 7.88(\mathrm{~d}, J=8.4 \mathrm{~Hz}, 1 \mathrm{H}), 7.75(\mathrm{~s}, 1 \mathrm{H}), 7.71(\mathrm{dd}, J=7.6$, $7.6 \mathrm{~Hz}, 1 \mathrm{H}), 7.52-7.49(\mathrm{~m}, 5 \mathrm{H}), 7.47-7.44(\mathrm{~m}, 3 \mathrm{H}) ;{ }^{13} \mathrm{C} \mathrm{NMR}\left(100 \mathrm{MHz}, \mathrm{CDCl}_{3}, \mathrm{ppm}\right) \delta 155.7,149.6$, $148.9,138.4,138.2,135.7,130.3,129.9,129.7,129.2,129.0,128.8,128.7,126.7,126.0,125.9,119.1$.

Xiao, F.; Chen, Y.; Liu, Yu.; Wang, J. Tetrahedron 2008, 64, 2755. 
<smiles>Brc1ccc(-c2cc(-c3ccccc3)c3ccccc3n2)cc1</smiles>

2-(4-bromophenyl)-4-phenylquinoline (4i): $2.95 \mathrm{~g}$ (82\% yield), ${ }^{1} \mathrm{H} \mathrm{NMR}\left(400 \mathrm{MHz}, \mathrm{CDCl}_{3}, \mathrm{ppm}\right) \delta$ $8.21(\mathrm{~d}, J=8.4 \mathrm{~Hz}, 1 \mathrm{H}), 8.06(\mathrm{~d}, J=8.4 \mathrm{~Hz}, 2 \mathrm{H}), 7.88(\mathrm{~d}, J=8.4 \mathrm{~Hz}, 1 \mathrm{H}), 7.75(\mathrm{~s}, 1 \mathrm{H}), 7.71(\mathrm{dd}, J=7.6$, $7.6 \mathrm{~Hz}, 1 \mathrm{H}), 7.62(\mathrm{~d}, J=8.4 \mathrm{~Hz}, 2 \mathrm{H}), 7.52-7.49(\mathrm{~m}, 5 \mathrm{H}), 7.46(\mathrm{dd}, J=7.6,7.6 \mathrm{~Hz}, 1 \mathrm{H}) ;{ }^{13} \mathrm{C} \mathrm{NMR}(100$ $\left.\mathrm{MHz}, \mathrm{CDCl}_{3}, \mathrm{ppm}\right) \delta 155.7,149.6,148.9,138.6,138.4,132.1,130.3,129.9,129.7,129.3,128.8,128.7$, 126.7, 126.0, 125.9, 124.1, 119.0.

Xiao, F.; Chen, Y.; Liu, Yu.; Wang, J. Tetrahedron 2008, 64, 2755.<smiles>FC(F)(F)c1ccc(-c2cc(-c3ccccc3)c3ccccc3n2)cc1</smiles>

4-phenyl-2-(4-(trifluoromethyl)phenyl)quinoline (4j): $2.40 \mathrm{~g}\left(69 \%\right.$ yield), ${ }^{1} \mathrm{H} \mathrm{NMR}\left(400 \mathrm{MHz}, \mathrm{CDCl}_{3}\right.$, ppm) $\delta 8.31(\mathrm{~d}, J=8.0 \mathrm{~Hz}, 2 \mathrm{H}), 8.24(\mathrm{~d}, J=8.4 \mathrm{~Hz}, 1 \mathrm{H}), 7.92(\mathrm{~d}, J=8.4 \mathrm{~Hz}, 1 \mathrm{H}), 7.81(\mathrm{~s}, 1 \mathrm{H}), 7.78-7.73$ (m, 3H), 7.56-7.50 (m, 6H); ${ }^{13} \mathrm{C}$ NMR (100 MHz, $\left.\mathrm{CDCl}_{3}, \mathrm{ppm}\right) \delta 155.4,149.8,149.0,143.1,138.3,131.3$ $\left(\mathrm{q}, J_{\mathrm{C}-\mathrm{F}}=32.3 \mathrm{~Hz}\right), 130.5,130.0,129.7,128.9,128.8,128.1,127.1,126.2,125.9\left(\mathrm{q}, J_{\mathrm{C}-\mathrm{F}}=3.2 \mathrm{~Hz}\right), 124.4$ $\left(\mathrm{q}, J_{\mathrm{C}-\mathrm{F}}=270.6 \mathrm{~Hz}\right), 119.3$.

Martínez, R.; Ramón, J. D.; Yus, M. Eur. J. Org. Chem. 2007, 10, 1599.<smiles>Cc1ccc(-c2cc(-c3ccccc3)c3ccccc3n2)cc1</smiles>

4-phenyl-2-(p-tolyl)quinoline (4k): $2.41 \mathrm{~g}$ (83\% yield), ${ }^{1} \mathrm{H} \mathrm{NMR}\left(400 \mathrm{MHz}, \mathrm{CDCl}_{3}, \mathrm{ppm}\right) \delta 8.22(\mathrm{~d}, J=$ $8.4 \mathrm{~Hz}, 1 \mathrm{H}), 8.09$ (d, $J=7.6 \mathrm{~Hz}, 2 \mathrm{H}), 7.88(\mathrm{~d}, J=8.4 \mathrm{~Hz}, 1 \mathrm{H}), 7.79(\mathrm{~s}, 1 \mathrm{H}), 7.70(\mathrm{dd}, J=7.6,7.6 \mathrm{~Hz}, 1 \mathrm{H})$, 7.53-7.47 (m, 5H), $7.43(\mathrm{dd}, J=7.6,7.6 \mathrm{~Hz}, 1 \mathrm{H}), 7.31(\mathrm{~d}, J=7.6 \mathrm{~Hz}, 2 \mathrm{H}), 2.41(\mathrm{~s}, 3 \mathrm{H}) ;{ }^{13} \mathrm{C}$ NMR $(100$ 
$\left.\mathrm{MHz}, \mathrm{CDCl}_{3}, \mathrm{ppm}\right) \delta 157.0,149.2,149.0,139.6,138.7,137.0,130.2,129.7,129.6,128.7,128.5,127.6$, $126.3,125.9,125.8,119.4,21.5$.

Chanda, T.; Verma, R. K.; Singh, M. S. Chem. Asian J. 2012, 7, 778.<smiles>c1ccc(-c2ccc(-c3cc(-c4ccccc4)c4ccccc4n3)cc2)cc1</smiles>

2-([1,1'-biphenyl]-4-yl)-4-phenylquinoline (4l): $2.32 \mathrm{~g}$ (65\% yield), ${ }^{1} \mathrm{H} \mathrm{NMR}\left(400 \mathrm{MHz}, \mathrm{CDCl}_{3}, \mathrm{ppm}\right) \delta$ 8.30-8.25 (m, 3H), $7.91(\mathrm{~d}, J=8.4 \mathrm{~Hz}, 1 \mathrm{H}), 7.87(\mathrm{~s}, 1 \mathrm{H}), 7.77-7.72(\mathrm{~m}, 3 \mathrm{H}), 7.68(\mathrm{~d}, J=8.0 \mathrm{~Hz}, 2 \mathrm{H})$, 7.59-7.52 (m, 5H), 7.50-7.46 (m, 3H), 7.39-7.36 (m, 1H); $\left.{ }^{13} \mathrm{C} \mathrm{NMR} \mathrm{(100} \mathrm{MHz,} \mathrm{CDCl}_{3}, \mathrm{ppm}\right) \delta 156.6$, $149.3,149.1,142.3,140.7,138.7,138.6,130.3,129.8,129.7,129.0,128.8,128.6,128.1,127.8,127.7$, $127.3,126.5,126.0,125.8,119.4$.

Xiao, F.; Chen, Y.; Liu, Yu.; Wang, J. Tetrahedron 2008, 64, 2755.<smiles>Cc1ccc(-c2cc(-c3ccccc3)c3ccccc3n2)cc1C</smiles>

2-(3,4-dimethylphenyl)-4-phenylquinoline (4m): 2.09 g (67\% yield), White solid, m.p. 113-116 ${ }^{\circ} \mathrm{C}$; IR (film): 1591, 1575, 1544, $1416 \mathrm{~cm}^{-1} ;{ }^{1} \mathrm{H} \mathrm{NMR}\left(400 \mathrm{MHz}, \mathrm{CDCl}_{3}, \mathrm{ppm}\right) \delta 8.23(\mathrm{~d}, J=8.4 \mathrm{~Hz}, 1 \mathrm{H}), 8.00$ (s, 1H), 7.89 (dd, $J=7.6,7.6 \mathrm{~Hz}, 2 \mathrm{H}), 7.80(\mathrm{~s}, 1 \mathrm{H}), 7.71(\mathrm{dd}, J=7.6,7.6 \mathrm{~Hz}, 1 \mathrm{H}), 7.55-7.49(\mathrm{~m}, 5 \mathrm{H}), 7.44$ $(\mathrm{dd}, J=7.6,7.6 \mathrm{~Hz}, 1 \mathrm{H}), 7.27(\mathrm{~d}, J=8.0 \mathrm{~Hz}, 1 \mathrm{H}), 2.38(\mathrm{~s}, 3 \mathrm{H}), 2.33(\mathrm{~s}, 3 \mathrm{H}) ;{ }^{13} \mathrm{C} \mathrm{NMR}\left(100 \mathrm{MHz}, \mathrm{CDCl}_{3}\right.$, ppm) $\delta 157.2,149.1,149.0,138.7,138.3,137.4,137.3,130.3,130.2,129.8,129.6,128.9,128.8,128.5$, 126.3, 125.9, 125.8, 125.2, 119.5, 20.2, 19.9; HRMS (ESI) calcd for $\mathrm{C}_{23} \mathrm{H}_{20} \mathrm{~N}(\mathrm{M}+\mathrm{H})$ : 310.1590 , Found: 310.1587 . 
<smiles>c1ccc(-c2cccc(-c3ccc4ccccc4c3)c2)cc1</smiles>

2-(naphthalen-2-yl)-4-phenylquinoline (4n): $1.85 \mathrm{~g}$ (56\% yield), ${ }^{1} \mathrm{H} \mathrm{NMR}\left(400 \mathrm{MHz}, \mathrm{CDCl}_{3}, \mathrm{ppm}\right) \delta$ $8.64(\mathrm{~s}, 1 \mathrm{H}), 8.41(\mathrm{~d}, J=8.4 \mathrm{~Hz}, 1 \mathrm{H}), 8.29(\mathrm{~d}, J=8.4 \mathrm{~Hz}, 1 \mathrm{H}), 8.00-7.98(\mathrm{~m}, 3 \mathrm{H}), 7.93-7.88(\mathrm{~m}, 2 \mathrm{H})$, $7.75(\mathrm{dd}, J=7.6,7.6 \mathrm{~Hz}, 1 \mathrm{H}), 7.61-7.47(\mathrm{~m}, 8 \mathrm{H}) ;{ }^{13} \mathrm{C} \mathrm{NMR}\left(100 \mathrm{MHz}, \mathrm{CDCl}_{3}, \mathrm{ppm}\right) \delta 156.9,149.4$, $149.1,138.7,137.1,134.1,133.7,130.3,129.8,129.0,128.9,128.84,128.79,128.6,127.9,127.4,126.9$, $126.6,126.5,126.0,125.9,125.3,119.7$.

Kulkarni, A.; Török, B. Green Chem. 2010, 12, 875.<smiles>c1ccc(-c2cc(-c3ccco3)c3ccccc3n2)cc1</smiles>

2-(furan-2-yl)-4-phenylquinoline (4o): $1.77 \mathrm{~g}\left(65 \%\right.$ yield), ${ }^{1} \mathrm{H} \mathrm{NMR}\left(400 \mathrm{MHz}, \mathrm{CDCl}_{3}, \mathrm{ppm}\right) \delta 8.19$ (d, $J=8.4 \mathrm{~Hz}, 1 \mathrm{H}), 7.84(\mathrm{~d}, J=8.4 \mathrm{~Hz}, 1 \mathrm{H}), 7.75(\mathrm{~s}, 1 \mathrm{H}), 7.69(\mathrm{ddd}, J=7.6,7.6,1.2 \mathrm{~Hz}, 1 \mathrm{H}), 7.60(\mathrm{~d}, J=$ $0.8 \mathrm{~Hz}, 1 \mathrm{H}), 7.53-7.47(\mathrm{~m}, 5 \mathrm{H}), 7.42(\mathrm{ddd}, J=7.6,7.6,0.8 \mathrm{~Hz}, 1 \mathrm{H}), 7.22(\mathrm{~d}, J=3.2 \mathrm{~Hz}, 1 \mathrm{H}), 6.57(\mathrm{dd}, J$ $=3.2,1.2 \mathrm{~Hz}, 1 \mathrm{H}) ;{ }^{13} \mathrm{C} \mathrm{NMR}\left(100 \mathrm{MHz}, \mathrm{CDCl}_{3}, \mathrm{ppm}\right) \delta 153.9,149.2,148.8,148.7,144.3,138.3,129.9$, $129.8,129.7,128.7,128.6,126.4,126.0,125.9,117.9,112.4,110.3$.

Chanda, T.; Verma, R. K.; Singh, M. S. Chem. Asian J. 2012, 7, 778.<smiles>c1ccc(-c2cc(-c3cccs3)nc3ccccc23)cc1</smiles>

4-phenyl-2-(thiophen-2-yl)quinoline (4p): $2.21 \mathrm{~g}$ (77\% yield), ${ }^{1} \mathrm{H} \mathrm{NMR}\left(400 \mathrm{MHz}, \mathrm{CDCl}_{3}, \mathrm{ppm}\right) \delta 8.14$ $(\mathrm{d}, J=8.4 \mathrm{~Hz}, 1 \mathrm{H}), 7.82(\mathrm{~d}, J=8.4 \mathrm{~Hz}, 1 \mathrm{H}), 7.71-7.70(\mathrm{~m}, 2 \mathrm{H}), 7.67(\mathrm{ddd}, J=7.6,7.6,1.2 \mathrm{~Hz}, 1 \mathrm{H})$, 7.53-7.48 (m, 5H), $7.44(\mathrm{~d}, J=4.8 \mathrm{~Hz}, 1 \mathrm{H}), 7.40(\mathrm{dd}, J=7.2,7.2 \mathrm{~Hz}, 1 \mathrm{H}), 7.12(\mathrm{dd}, J=4.8,4.0 \mathrm{~Hz}, 1 \mathrm{H})$; 
${ }^{13} \mathrm{C} \mathrm{NMR}\left(100 \mathrm{MHz}, \mathrm{CDCl}_{3}, \mathrm{ppm}\right) \delta 152.0,149.2,148.8,145.6,138.3,129.8,129.7,128.8,128.7,128.6$, $128.3,126.3,126.1,126.0,125.8,118.1$.

Giridhar, T.; Han, T.-H.; Cho, W.; Saravanan, C.; Lee, T.-W.; Jin, S.-H. Chem. Eur. J. 2014, 20, 8260.<smiles>c1ccc(-c2cc(-c3ccsc3)nc3ccccc23)cc1</smiles>

4-phenyl-2-(thiophen-3-yl)quinoline (4q): $2.04 \mathrm{~g}$ (71\% yield), ${ }^{1} \mathrm{H} \mathrm{NMR}\left(400 \mathrm{MHz}, \mathrm{CDCl}_{3}, \mathrm{ppm}\right) \delta 8.17$ $(\mathrm{d}, J=8.4 \mathrm{~Hz}, 1 \mathrm{H}), 8.04(\mathrm{dd}, J=2.8,1.2 \mathrm{~Hz}, 1 \mathrm{H}), 7.88(\mathrm{dd}, J=5.2,0.8 \mathrm{~Hz}, 1 \mathrm{H}), 7.85(\mathrm{~d}, J=8.0 \mathrm{~Hz}, 1 \mathrm{H})$, 7.71-7.67 (m, 2H), 7.54-7.48 (m, 5H), 7.45-7.41 (m, 2H); $\left.{ }^{13} \mathrm{C} \mathrm{NMR} \mathrm{(100} \mathrm{MHz,} \mathrm{CDCl}_{3}, \mathrm{ppm}\right) \delta 153.0$, $149.2,149.0,142.9,138.5,130.1,129.7,128.8,128.6,127.0,126.5,126.3,125.9,125.8,124.9,119.5$.

Cao, K.; Zhang, F.-M.; Tu, Y.-Q.; Zhuo, X.-T.; Fan, C.-A. Chem. Eur. J. 2009, 15, 6332.<smiles>Fc1ccc(-c2cc(-c3ccccc3)nc3ccccc23)cc1</smiles>

4-(4-fluorophenyl)-2-phenylquinoline (4r): $1.76 \mathrm{~g}$ (59\% yield), ${ }^{1} \mathrm{H} \mathrm{NMR}\left(400 \mathrm{MHz}, \mathrm{CDCl}_{3}, \mathrm{ppm}\right) \delta$ $8.24(\mathrm{~d}, J=8.4 \mathrm{~Hz}, 1 \mathrm{H}), 8.17(\mathrm{~d}, J=7.6 \mathrm{~Hz}, 2 \mathrm{H}), 7.83(\mathrm{~d}, J=8.4 \mathrm{~Hz}, 1 \mathrm{H}), 7.76(\mathrm{~s}, 1 \mathrm{H}), 7.71(\mathrm{dd}, J=7.2$, 7.2 Hz, 1H), 7.52-7.42 (m, 6H), 7.23-7.19 (m, 2H); ${ }^{13} \mathrm{C} \mathrm{NMR}\left(100 \mathrm{MHz}, \mathrm{CDCl}_{3}, \mathrm{ppm}\right) \delta 163.1\left(\mathrm{~d}, J_{\mathrm{C}-\mathrm{F}}=\right.$ $246.5 \mathrm{~Hz}), 157.0,149.0,148.2,139.7,134.5\left(\mathrm{~d}, J_{\mathrm{C}-\mathrm{F}}=3.4 \mathrm{~Hz}\right), 131.4\left(\mathrm{~d}, J_{\mathrm{C}-\mathrm{F}}=8.0 \mathrm{~Hz}\right), 130.4,129.8$, $129.6,129.0,127.7,126.6,125.9,125.5,119.5,115.8\left(\mathrm{~d}, J_{\mathrm{C}-\mathrm{F}}=21.4 \mathrm{~Hz}\right)$.

Kulkarni, A.; Török, B. Green Chem. 2010, 12, 875. 
<smiles>Clc1ccc(-c2cc(-c3ccccc3)nc3ccccc23)cc1</smiles>

4-(4-chlorophenyl)-2-phenylquinoline (4s): $1.95 \mathrm{~g}$ (62\% yield), ${ }^{1} \mathrm{H} \mathrm{NMR}\left(400 \mathrm{MHz}, \mathrm{CDCl}_{3}, \mathrm{ppm}\right) \delta$ $8.23(\mathrm{~d}, J=8.4 \mathrm{~Hz}, 1 \mathrm{H}), 8.17(\mathrm{~d}, J=7.2 \mathrm{~Hz}, 2 \mathrm{H}), 7.82(\mathrm{~d}, J=8.4 \mathrm{~Hz}, 1 \mathrm{H}), 7.76(\mathrm{~s}, 1 \mathrm{H}), 7.71(\mathrm{dd}, J=7.6$, $7.6 \mathrm{~Hz}, 1 \mathrm{H}), 7.53-7.45(\mathrm{~m}, 8 \mathrm{H}) ;{ }^{13} \mathrm{C} \mathrm{NMR}\left(100 \mathrm{MHz}, \mathrm{CDCl}_{3}, \mathrm{ppm}\right) \delta 157.0,149.0,148.0,139.7,137.0$, $134.8,131.1,130.5,129.9,129.6,129.1,127.7,126.7,125.7,125.4,119.4$.

Cao, K.; Zhang, F.-M.; Tu, Y.-Q.; Zhuo, X.-T.; Fan, C.-A. Chem. Eur. J. 2009, 15, 6332.<smiles>Brc1ccc(-c2cc(-c3ccccc3)nc3ccccc23)cc1</smiles>

4-(4-bromophenyl)-2-phenylquinoline (4t): $2.50 \mathrm{~g}$ (68\% yield), ${ }^{1} \mathrm{H} \mathrm{NMR}\left(400 \mathrm{MHz}, \mathrm{CDCl}_{3}, \mathrm{ppm}\right) \delta$ $8.24(\mathrm{~d}, J=8.4 \mathrm{~Hz}, 1 \mathrm{H}), 8.18(\mathrm{~d}, J=7.6 \mathrm{~Hz}, 2 \mathrm{H}), 7.83(\mathrm{~d}, J=8.4 \mathrm{~Hz}, 1 \mathrm{H}), 7.77(\mathrm{~s}, 1 \mathrm{H}), 7.73(\mathrm{dd}, J=7.6$, $7.6 \mathrm{~Hz}, 1 \mathrm{H}), 7.67(\mathrm{~d}, J=8.0 \mathrm{~Hz}, 2 \mathrm{H}), 7.54-7.46(\mathrm{~m}, 4 \mathrm{H}), 7.42(\mathrm{~d}, J=8.0 \mathrm{~Hz}, 2 \mathrm{H}) ;{ }^{13} \mathrm{C} \mathrm{NMR}(100 \mathrm{MHz}$, $\left.\mathrm{CDCl}_{3}, \mathrm{ppm}\right) \delta 157.1,149.0,148.1,139.7,137.5,132.0,131.4,130.5,129.9,129.7,129.1,127.8,126.8$, $125.6,125.4,123.0,119.4$.

Zhu, X.; Wang, Y.-F.; Zhang, F.-L.; Chiba, S. Chem. Asian J. 2014, 9, 2458.<smiles>Cc1ccc(-c2cc(-c3ccccc3)nc3ccccc23)cc1</smiles>

2-phenyl-4-(p-tolyl)quinoline (4u): $2.36 \mathrm{~g}\left(80 \%\right.$ yield), ${ }^{1} \mathrm{H} \mathrm{NMR}\left(300 \mathrm{MHz}, \mathrm{CDCl}_{3}, \mathrm{ppm}\right) \delta 8.23$ (d, $J=$ $8.4 \mathrm{~Hz}, 1 \mathrm{H}), 8.18(\mathrm{~d}, J=7.6 \mathrm{~Hz}, 2 \mathrm{H}), 7.92(\mathrm{~d}, J=8.4 \mathrm{~Hz}, 1 \mathrm{H}), 7.79(\mathrm{~s}, 1 \mathrm{H}), 7.70$ (ddd, $J=7.6,7.6,1.2$ 
$\mathrm{Hz}, 1 \mathrm{H}), 7.52-7.49(\mathrm{~m}, 2 \mathrm{H}), 7.45-7.43(\mathrm{~m}, 4 \mathrm{H}), 7.34-7.33(\mathrm{~m}, 2 \mathrm{H}), 2.46(\mathrm{~s}, 3 \mathrm{H}) ;{ }^{13} \mathrm{C} \mathrm{NMR}(75 \mathrm{MHz}$, $\left.\mathrm{CDCl}_{3}, \mathrm{ppm}\right) \delta 157.1,149.4,149.1,139.9,138.5,135.7,130.3,129.7,129.6,129.5,129.0,127.8,126.4$, $126.1,125.9,119.5,21.5$.

Zhu, X.; Wang, Y.-F.; Zhang, F.-L.; Chiba, S. Chem. Asian J. 2014, 9, 2458.<smiles>COc1ccc(-c2cc(-c3ccccc3)nc3ccccc23)cc1</smiles>

4-(4-methoxyphenyl)-2-phenylquinoline (4v): $2.34 \mathrm{~g}$ (75\% yield), ${ }^{1} \mathrm{H} \mathrm{NMR}\left(300 \mathrm{MHz}, \mathrm{CDCl}_{3}, \mathrm{ppm}\right) \delta$ $8.23(\mathrm{~d}, J=8.4 \mathrm{~Hz}, 1 \mathrm{H}), 8.18(\mathrm{~d}, J=7.2 \mathrm{~Hz}, 2 \mathrm{H}), 7.93(\mathrm{~d}, J=8.4 \mathrm{~Hz}, 1 \mathrm{H}), 7.79(\mathrm{~s}, 1 \mathrm{H}), 7.71(\mathrm{dd}, J=7.6$, 7.6 Hz, 1H), 7.53-7.45 (m, 6H), 7.07 (d, $J=8.4 \mathrm{~Hz}, 2 \mathrm{H}), 3.89(\mathrm{~s}, 3 \mathrm{H}) ;{ }^{13} \mathrm{C} \mathrm{NMR}\left(75 \mathrm{MHz}, \mathrm{CDCl}_{3}, \mathrm{ppm}\right)$ $\delta 160.1,157.1,149.10,149.06,140.0,131.0,130.9,130.3,129.6,129.5,129.0,127.8,126.4,126.2,125.9$, $119.5,114.3,55.6$.

Cao, K.; Zhang, F.-M.; Tu, Y.-Q.; Zhuo, X.-T.; Fan, C.-A. Chem. Eur. J. 2009, 15, 6332.<smiles>C1=C(c2cc(-c3ccccc3)nc3ccccc23)CCCC1</smiles>

4-(cyclohex-1-en-1-yl)-2-phenylquinoline (4w): $1.08 \mathrm{~g}$ (38\% yield), ${ }^{1} \mathrm{H} \mathrm{NMR}\left(300 \mathrm{MHz}, \mathrm{CDCl}_{3}, \mathrm{ppm}\right)$ $\delta 8.18-8.15(\mathrm{~m}, 3 \mathrm{H}), 8.00(\mathrm{~d}, J=8.0 \mathrm{~Hz}, 1 \mathrm{H}), 7.69-7.65(\mathrm{~m}, 2 \mathrm{H}), 7.52-7.41(\mathrm{~m}, 4 \mathrm{H}), 5.89-5.87(\mathrm{~m}, 1 \mathrm{H})$,

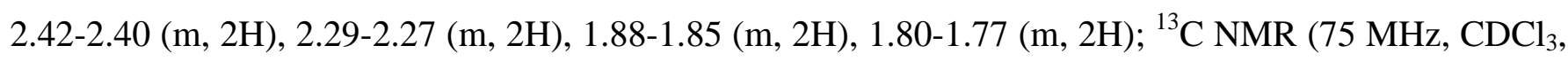
ppm) $\delta 157.2,152.2,148.9,140.1,136.0,130.3,129.4,129.3,129.0,128.9,127.7,126.0,125.9,125.6$, $117.7,30.5,25.6,23.1,22.3$.

Cao, K.; Zhang, F.-M.; Tu, Y.-Q.; Zhuo, X.-T.; Fan, C.-A. Chem. Eur. J. 2009, 15, 6332. 
<smiles>Cc1ccc2nc(-c3ccccc3)cc(-c3ccccc3)c2c1</smiles>

6-methyl-2,4-diphenylquinoline (4x): $2.45 \mathrm{~g}$ (83\% yield), ${ }^{1} \mathrm{H} \mathrm{NMR}\left(400 \mathrm{MHz}, \mathrm{CDCl}_{3}, \mathrm{ppm}\right) \delta 8.17-8.12$ $(\mathrm{m}, 3 \mathrm{H}), 7.77(\mathrm{~s}, 1 \mathrm{H}), 7.64(\mathrm{~s}, 1 \mathrm{H}), 7.57-7.49(\mathrm{~m}, 8 \mathrm{H}), 7.46-7.42(\mathrm{~m}, 1 \mathrm{H}), 2.47(\mathrm{~s}, 3 \mathrm{H}) ;{ }^{13} \mathrm{C}$ NMR $(125$ $\left.\mathrm{MHz}, \mathrm{CDCl}_{3}, \mathrm{ppm}\right) \delta 156.2,148.6,147.6,140.0,138.9,136.5,132.0,130.1,129.8,129.4,129.0,128.8$, $128.5,127.7,125.9,124.6,119.6,22.0$.

Cao, K.; Zhang, F.-M.; Tu, Y.-Q.; Zhuo, X.-T.; Fan, C.-A. Chem. Eur. J. 2009, 15, 6332.<smiles>Clc1ccc2nc(-c3ccccc3)cc(-c3ccccc3)c2c1</smiles>

6-chloro-2,4-diphenylquinoline (4y): $1.92 \mathrm{~g}$ (61\% yield), ${ }^{1} \mathrm{H} \mathrm{NMR}\left(400 \mathrm{MHz}, \mathrm{CDCl}_{3}, \mathrm{ppm}\right) \delta 8.17-8.13$ $(\mathrm{m}, 3 \mathrm{H}), 7.84(\mathrm{~s}, 1 \mathrm{H}), 7.79(\mathrm{~s}, 1 \mathrm{H}), 7.61(\mathrm{dd}, J=8.8,1.6 \mathrm{~Hz}, 1 \mathrm{H}), 7.54-7.42(\mathrm{~m}, 8 \mathrm{H}) ;{ }^{13} \mathrm{C} \mathrm{NMR}(100$ $\left.\mathrm{MHz}, \mathrm{CDCl}_{3}, \mathrm{ppm}\right) \delta 157.1,148.5,147.4,139.3,137.9,132.3,131.9,130.6,129.7,129.6,129.03,128.96$, $128.8,127.7,126.6,124.6,120.1$.

Chanda, T.; Verma, R. K.; Singh, M. S. Chem. Asian J. 2012, 7, 778.<smiles>c1ccc(C2CC(c3ccccc3)c3ccccc3N2)cc1</smiles>

(-)-cis-2,4-diphenyl-1,2,3,4-tetrahydroquinoline (5a): $0.1037 \mathrm{~g}(91 \%$ yield $),[\alpha]_{\mathrm{D}}{ }^{20}=-109.9(c 0.72$, $\left.\mathrm{CH}_{2} \mathrm{Cl}_{2}\right)(91 \%$ ee $)\left[\right.$ lit: $[\alpha]_{\mathrm{D}}{ }^{25}=-111.2\left(c 0.52, \mathrm{CHCl}_{3}\right)(>95: 5$ d.r, 98\% ee $\left.)\right] ;{ }^{1} \mathrm{H} \mathrm{NMR}\left(400 \mathrm{MHz}, \mathrm{CDCl}_{3}\right.$, ppm) $\delta 7.43(\mathrm{~d}, J=7.2 \mathrm{~Hz}, 2 \mathrm{H}), 7.35-7.26(\mathrm{~m}, 5 \mathrm{H}), 7.24-7.19(\mathrm{~m}, 3 \mathrm{H}), 6.99(\mathrm{dd}, J=7.6,7.6 \mathrm{~Hz}, 1 \mathrm{H})$, $6.63(\mathrm{~d}, J=8.0 \mathrm{~Hz}, 1 \mathrm{H}), 6.55(\mathrm{dd}, J=7.6,6.8 \mathrm{~Hz}, 2 \mathrm{H}), 4.58(\mathrm{dd}, J=10.8,2.8 \mathrm{~Hz}, 1 \mathrm{H}), 4.28(\mathrm{dd}, J=12.0$, $5.6 \mathrm{~Hz}, 1 \mathrm{H}), 4.02(\mathrm{~s}, 1 \mathrm{H}), 2.29-2.15(\mathrm{~m}, 2 \mathrm{H}) ;{ }^{13} \mathrm{C} \mathrm{NMR}\left(100 \mathrm{MHz}, \mathrm{CDCl}_{3}, \mathrm{ppm}\right) \delta 145.53,145.50,144.1$, $129.9,128.9,128.8,128.7,127.9,127.4,126.8,126.7,124.9,117.8,114.5,57.5,45.1,42.3$. 
Li, H. Y.; Horn, J.; Campbell, A.; House, D.; Nelson, A.; Marsden, S. P. Chem. Commun. 2014, 50, 10222.<smiles>Clc1ccccc1C1CC(c2ccccc2)c2ccccc2N1</smiles>

(-)-cis-2-(2-chlorophenyl)-4-phenyl-1,2,3,4-tetrahydroquinoline (5b): 0.1244 g (98\% yield), White solid, m.p. $69-72{ }^{\circ} \mathrm{C} ;[\alpha]_{\mathrm{D}}{ }^{20}=-163.6\left(c 0.89, \mathrm{CH}_{2} \mathrm{Cl}_{2}\right)\left(97 \%\right.$ ee); IR (film): $3368,1604,1586,1477 \mathrm{~cm}^{-1}$; ${ }^{1} \mathrm{H}$ NMR $\left(400 \mathrm{MHz}, \mathrm{CDCl}_{3}, \mathrm{ppm}\right) \delta 7.69(\mathrm{dd}, J=7.6,1.2 \mathrm{~Hz}, 1 \mathrm{H}), 7.36-7.17(\mathrm{~m}, 8 \mathrm{H}), 7.01(\mathrm{dd}, J=7.6$, $7.6 \mathrm{~Hz}, 1 \mathrm{H}), 6.65(\mathrm{~d}, J=7.6 \mathrm{~Hz}, 1 \mathrm{H}), 6.61-6.56(\mathrm{~m}, 2 \mathrm{H}), 5.08(\mathrm{dd}, J=11.2,2.0 \mathrm{~Hz}, 1 \mathrm{H}), 4.33(\mathrm{dd}, J=$ 12.4, $5.2 \mathrm{~Hz}, 1 \mathrm{H}), 3.97(\mathrm{~s}, 1 \mathrm{H}), 2.43-2.39(\mathrm{~m}, 1 \mathrm{H}), 2.06(\mathrm{~m}, \mathrm{~Hz}, 1 \mathrm{H}) ;{ }^{13} \mathrm{C} \mathrm{NMR}\left(100 \mathrm{MHz}, \mathrm{CDCl}_{3}, \mathrm{ppm}\right)$ $\delta 145.6,145.4,141.1,133.0,130.0,129.8,128.9,128.8,128.7,127.6,127.4,126.7,125.2,118.1,114.9$, 53.2, 44.9, 40.1; HRMS (ESI) calcd for $\mathrm{C}_{21} \mathrm{H}_{19} \mathrm{NCl}(\mathrm{M}+\mathrm{H})$ : 320.1201 , Found: 320.1197.<smiles>Brc1ccccc1C1CC(c2ccccc2)c2ccccc2N1</smiles>

(-)-cis-2-(2-bromophenyl)-4-phenyl-1,2,3,4-tetrahydroquinoline (5c): $0.1247 \mathrm{~g}$ (86\% yield), White solid, m.p. $73-76{ }^{\circ} \mathrm{C} ;[\alpha]_{\mathrm{D}}{ }^{20}=-219.7\left(c 0.70, \mathrm{CH}_{2} \mathrm{Cl}_{2}\right)\left(97 \%\right.$ ee); IR (film): $3375,1603,1585,1480 \mathrm{~cm}^{-1}$; ${ }^{1} \mathrm{H}$ NMR (400 MHz, $\left.\mathrm{CDCl}_{3}, \mathrm{ppm}\right) \delta 7.66(\mathrm{~d}, J=7.6 \mathrm{~Hz}, 1 \mathrm{H}), 7.52(\mathrm{~d}, J=8.0 \mathrm{~Hz}, 1 \mathrm{H}), 7.33-7.27(\mathrm{~m}, 3 \mathrm{H})$, 7.24-7.18 (m, 3H), 7.10 (ddd, $J=7.6,7.6,1.2 \mathrm{~Hz}, 1 \mathrm{H}), 7.00(\mathrm{dd}, J=7.6,7.6 \mathrm{~Hz}, 1 \mathrm{H}), 6.65(\mathrm{~d}, J=7.6 \mathrm{~Hz}$, $1 \mathrm{H}), 6.57(\mathrm{~d}, J=8.0,8.0 \mathrm{~Hz}, 2 \mathrm{H}), 5.01(\mathrm{dd}, J=10.8,2.0 \mathrm{~Hz}, 1 \mathrm{H}), 4.31(\mathrm{dd}, J=12.4,5.2 \mathrm{~Hz}, 1 \mathrm{H}), 3.95(\mathrm{~s}$, 1H), 2.43-2.19 (m, 1H), $2.02(\mathrm{~m}, \mathrm{~Hz}, 1 \mathrm{H}) ;{ }^{13} \mathrm{C} \mathrm{NMR}\left(100 \mathrm{MHz}, \mathrm{CDCl}_{3}, \mathrm{ppm}\right) \delta 145.5,145.4,142.6$, $133.1,129.9,129.1,128.9,128.7,128.2,127.8,127.4,126.7,125.2,123.3,118.1,114.9,55.8,44.9,40.2$; HRMS (ESI) calcd for $\mathrm{C}_{21} \mathrm{H}_{19} \mathrm{NBr}(\mathrm{M}+\mathrm{H}): 364.0695$, Found: 364.0691. 
<smiles>Clc1cccc(C2CC(c3ccccc3)c3ccccc3N2)c1</smiles>

(-)-cis-2-(3-chlorophenyl)-4-phenyl-1,2,3,4-tetrahydroquinoline (5d): $0.1175 \mathrm{~g}$ (92\% yield), White solid, m.p. $61-64{ }^{\circ} \mathrm{C} ;[\alpha]_{\mathrm{D}}{ }^{20}=-79.7\left(c 0.65, \mathrm{CH}_{2} \mathrm{Cl}_{2}\right)\left(90 \%\right.$ ee); IR (film): $3386,1599,1586,1477 \mathrm{~cm}^{-1} ;{ }^{1} \mathrm{H}$ NMR (400 MHz, $\left.\mathrm{CDCl}_{3}, \mathrm{ppm}\right) \delta 7.46(\mathrm{~s}, 1 \mathrm{H}), 7.32-7.28(\mathrm{~m}, 3 \mathrm{H}), 7.26-7.20(\mathrm{~m}, 5 \mathrm{H}), 7.01(\mathrm{dd}, J=7.6$, $7.6 \mathrm{~Hz}, 1 \mathrm{H}), 6.64(\mathrm{~d}, J=8.0 \mathrm{~Hz}, 1 \mathrm{H}), 6.57(\mathrm{dd}, J=8.0,6.4 \mathrm{~Hz}, 2 \mathrm{H}), 4.57(\mathrm{dd}, J=11.2,2.8 \mathrm{~Hz}, 1 \mathrm{H}), 4.28$ $(\mathrm{dd}, J=12.0,5.6 \mathrm{~Hz}, 1 \mathrm{H}), 4.02(\mathrm{~s}, 1 \mathrm{H}), 2.29-2.25(\mathrm{~m}, 1 \mathrm{H}), 2.17(\mathrm{~m}, 1 \mathrm{H}) ;{ }^{13} \mathrm{C} \mathrm{NMR}\left(100 \mathrm{MHz}, \mathrm{CDCl}_{3}\right.$, ppm) $\delta 146.3,145.3,145.2,134.7,130.1,129.9,128.9,128.8,128.1,127.5,127.0,126.8,125.0,124.9$, 118.1, 114.6, 57.0, 45.0, 42.3. HRMS (ESI) calcd for $\mathrm{C}_{21} \mathrm{H}_{19} \mathrm{NCl}(\mathrm{M}+\mathrm{H})$ : 320.1201, Found: 320.1197.<smiles>Brc1cccc(C2CC(c3ccccc3)c3ccccc3N2)c1</smiles>

(-)-cis-2-(3-bromophenyl)-4-phenyl-1,2,3,4-tetrahydroquinoline (5e): $0.1301 \mathrm{~g}$ (89\% yield), White solid, m.p. $83-86^{\circ} \mathrm{C} ;[\alpha]_{\mathrm{D}}{ }^{20}=-67.0\left(c 0.67, \mathrm{CH}_{2} \mathrm{Cl}_{2}\right)\left(91 \%\right.$ ee); IR (film): $3390,1605,1586,1479 \mathrm{~cm}^{-1} ;{ }^{1} \mathrm{H}$ NMR (400 MHz, $\left.\mathrm{CDCl}_{3}, \mathrm{ppm}\right) \delta$ 7.62-7.61 (m, 1H), $7.39(\mathrm{~d}, J=8.0 \mathrm{~Hz}, 1 \mathrm{H}), 7.34(\mathrm{~d}, J=7.6 \mathrm{~Hz}, 1 \mathrm{H})$, 7.31-7.28 (m, 2H), 7.23-7.19 (m, 4H), 7.00 (dd, $J=7.6,7.6 \mathrm{~Hz}, 1 \mathrm{H}), 6.63(\mathrm{~d}, J=7.6 \mathrm{~Hz}, 1 \mathrm{H}), 6.58-6.55$ $(\mathrm{m}, 2 \mathrm{H}), 4.54(\mathrm{dd}, J=11.2,2.8 \mathrm{~Hz}, 1 \mathrm{H}), 4.27(\mathrm{dd}, J=12.0,5.6 \mathrm{~Hz}, 1 \mathrm{H}), 4.00(\mathrm{~s}, 1 \mathrm{H}), 2.28-2.24(\mathrm{~m}, 1 \mathrm{H})$, $2.15(\mathrm{~m}, 1 \mathrm{H}) ;{ }^{13} \mathrm{C} \mathrm{NMR}\left(100 \mathrm{MHz}, \mathrm{CDCl}_{3}, \mathrm{ppm}\right) \delta 146.5,145.23,145.17,131.0,130.4,129.9,128.9$, 128.8, 127.5, 126.8, 125.5, 124.9, 122.9, 118.1, 114.6, 57.0, 45.0, 42.3; HRMS (ESI) calcd for $\mathrm{C}_{21} \mathrm{H}_{19} \mathrm{NBr}$ (M+H): 364.0695, Found: 364.0690 .<smiles>Cc1cccc(C2CC(c3ccccc3)c3ccccc3N2)c1</smiles> 
(-)-cis-4-phenyl-2-(m-tolyl)-1,2,3,4-tetrahydroquinoline (5f): 0.1043 g (87\% yield), Colorless oil; $[\alpha]_{\mathrm{D}}^{20}=-110.9\left(c 0.71, \mathrm{CH}_{2} \mathrm{Cl}_{2}\right)(91 \%$ ee $)$; IR (film): 3382, 1605, 1479, $1453 \mathrm{~cm}^{-1} ;{ }^{1} \mathrm{H}$ NMR (400 MHz, $\left.\mathrm{CDCl}_{3}, \mathrm{ppm}\right) \delta$ 7.31-7.26 (m, 3H), 7.24-7.17 (m, 5H), 7.09-7.07 (m, 1H), $6.99(\mathrm{dd}, J=7.6,7.6 \mathrm{~Hz}, 1 \mathrm{H})$, $6.63(\mathrm{~d}, J=7.6 \mathrm{~Hz}, 1 \mathrm{H}), 6.56-6.53(\mathrm{~m}, 2 \mathrm{H}), 4.55(\mathrm{dd}, J=10.8,3.2 \mathrm{~Hz}, 1 \mathrm{H}), 4.28(\mathrm{dd}, J=12.0,6.0 \mathrm{~Hz}$, 1H), 4.01 (s, 1H), 2.34 (s, 3H), 2.30-2.15 (m, 2H); $\left.{ }^{13} \mathrm{C} \mathrm{NMR} \mathrm{(100} \mathrm{MHz,} \mathrm{CDCl}_{3}, \mathrm{ppm}\right) \delta 145.56,145.55$, $144.0,138.5,129.9,128.9,128.73,128.70,128.6,127.44,127.40,126.7,124.9,123.9,117.7,114.4,57.4$, 45.2, 42.2, 21.6; HRMS (ESI) calcd for $\mathrm{C}_{22} \mathrm{H}_{22} \mathrm{~N}(\mathrm{M}+\mathrm{H})$ : 300.1747, Found: 300.1744 .<smiles>COc1cccc(C2CC(c3ccccc3)c3ccccc3N2)c1</smiles>

(-)-cis-2-(3-methoxyphenyl)-4-phenyl-1,2,3,4-tetrahydroquinoline $\quad(5 \mathrm{~g}): 0.1172$ g $\quad$ (93\% yield), Colorless oil; $[\alpha]_{\mathrm{D}}{ }^{20}=-91.9\left(c 0.66, \mathrm{CH}_{2} \mathrm{Cl}_{2}\right)\left(94 \%\right.$ ee); IR (film): $3377,1603,1586,1483 \mathrm{~cm}^{-1} ;{ }^{1} \mathrm{H}$ NMR (400 MHz, $\left.\mathrm{CDCl}_{3}, \mathrm{ppm}\right) \delta$ 7.33-7.28 (m, 2H), 7.26-7.21 (m, 4H), 7.03-6.99 (m, 3H), 6.84-6.81 (m, 1H), $6.64(\mathrm{~d}, J=7.6 \mathrm{~Hz}, 1 \mathrm{H}), 6.59-6.54(\mathrm{~m}, 2 \mathrm{H}), 4.59(\mathrm{dd}, J=11.2,2.8 \mathrm{~Hz}, 1 \mathrm{H}), 4.30(\mathrm{dd}, J=12.0,5.6 \mathrm{~Hz}$, $1 \mathrm{H}), 4.07(\mathrm{~s}, 1 \mathrm{H}), 3.81(\mathrm{~s}, 3 \mathrm{H}), 2.31-2.28(\mathrm{~m}, 1 \mathrm{H}), 2.21(\mathrm{~m}, 1 \mathrm{H}) ;{ }^{13} \mathrm{C} \mathrm{NMR}\left(100 \mathrm{MHz}, \mathrm{CDCl}_{3}, \mathrm{ppm}\right) \delta$ $160.2,145.8,145.50,145.48,129.9,128.9,128.7,127.5,126.7,124.9,119.2,117.8,114.5,113.5,112.2$, 57.5, 55.5, 45.1, 42.3; HRMS (ESI) calcd for $\mathrm{C}_{22} \mathrm{H}_{22} \mathrm{NO}(\mathrm{M}+\mathrm{H}): 316.1696$, Found: 316.1693.<smiles>Clc1ccc(C2CC(c3ccccc3)c3ccccc3N2)cc1</smiles>

(-)-cis-2-(4-chlorophenyl)-4-phenyl-1,2,3,4-tetrahydroquinoline (5h): 0.1159 g (91\% yield), White solid, m.p. $149-151{ }^{\circ} \mathrm{C} ;[\alpha]_{\mathrm{D}}{ }^{20}=-41.6\left(c 0.64, \mathrm{CH}_{2} \mathrm{Cl}_{2}\right)\left(91 \%\right.$ ee); IR (film): $3391,1645,1605,1480 \mathrm{~cm}^{-1}$; ${ }^{1} \mathrm{H}$ NMR (400 MHz, $\left.\mathrm{CDCl}_{3}, \mathrm{ppm}\right) \delta 7.37(\mathrm{~d}, J=8.4 \mathrm{~Hz}, 2 \mathrm{H}), 7.31-7.28(\mathrm{~m}, 4 \mathrm{H}), 7.23-7.20(\mathrm{~m}, 3 \mathrm{H}), 7.00$ $(\mathrm{dd}, J=7.6,7.6 \mathrm{~Hz}, 1 \mathrm{H}), 6.63(\mathrm{~d}, J=7.6 \mathrm{~Hz}, 1 \mathrm{H}), 6.57(\mathrm{dd}, J=7.6,6.0 \mathrm{~Hz}, 2 \mathrm{H}), 4.56(\mathrm{dd}, J=11.2,2.8$ $\mathrm{Hz}, 1 \mathrm{H}), 4.28(\mathrm{dd}, J=12.0,5.6 \mathrm{~Hz}, 1 \mathrm{H}), 3.99(\mathrm{~s}, 1 \mathrm{H}), 2.27-2.22(\mathrm{~m}, 1 \mathrm{H}), 2.15(\mathrm{~m}, 1 \mathrm{H}) ;{ }^{13} \mathrm{C} \mathrm{NMR}(100$ 
$\left.\mathrm{MHz}, \mathrm{CDCl}_{3}, \mathrm{ppm}\right) \delta 145.31,145.29,142.6,133.5,129.9,129.0,128.9,128.8,128.2,127.5,126.8,124.9$, 118.1, 114.6, 56.9, 45.0, 42.3; HRMS (ESI) calcd for $\mathrm{C}_{21} \mathrm{H}_{19} \mathrm{NCl}(\mathrm{M}+\mathrm{H})$ : 320.1201, Found: 320.1198 .<smiles>Brc1ccc(C2CC(c3ccccc3)c3ccccc3N2)cc1</smiles>

(-)-cis-2-(4-bromophenyl)-4-phenyl-1,2,3,4-tetrahydroquinoline (5i): 0.1252 g (86\% yield), White solid, m.p. $161-163{ }^{\circ} \mathrm{C} ;[\alpha]_{\mathrm{D}}{ }^{20}=-114.1\left(c 0.73, \mathrm{CH}_{2} \mathrm{Cl}_{2}\right)\left(96 \%\right.$ ee); IR (film): $3379,1604,1583,1479 \mathrm{~cm}^{-1}$; ${ }^{1} \mathrm{H}$ NMR (400 MHz, $\left.\mathrm{CDCl}_{3}, \mathrm{ppm}\right) \delta 7.47(\mathrm{~d}, J=8.4 \mathrm{~Hz}, 2 \mathrm{H}), 7.34-7.29(\mathrm{~m}, 4 \mathrm{H}), 7.25-7.21(\mathrm{~m}, 3 \mathrm{H}), 7.01$ $(\mathrm{dd}, J=7.6,7.6 \mathrm{~Hz}, 1 \mathrm{H}), 6.64(\mathrm{~d}, J=8.0 \mathrm{~Hz}, 1 \mathrm{H}), 6.59-6.56(\mathrm{~m}, 2 \mathrm{H}), 4.57(\mathrm{dd}, J=11.2,2.8 \mathrm{~Hz}, 1 \mathrm{H})$, $4.29(\mathrm{dd}, J=12.0,5.6 \mathrm{~Hz}, 1 \mathrm{H}), 4.01(\mathrm{~s}, 1 \mathrm{H}), 2.28-2.23(\mathrm{~m}, 1 \mathrm{H}), 2.16(\mathrm{~m}, 1 \mathrm{H}) ;{ }^{13} \mathrm{C} \mathrm{NMR}(100 \mathrm{MHz}$, $\left.\mathrm{CDCl}_{3}, \mathrm{ppm}\right) \delta 145.31,145.29,143.2,129.9,128.9,128.8,128.6,127.5,126.8,124.9,121.6,118.1,114.6$ 57.0, 45.1, 42.3; HRMS (ESI) calcd for $\mathrm{C}_{21} \mathrm{H}_{19} \mathrm{NBr}(\mathrm{M}+\mathrm{H})$ : 364.0695, Found: 364.0690.<smiles>FC(F)(F)c1ccc(C2CC(c3ccccc3)c3ccccc3N2)cc1</smiles>

(-)-cis-4-phenyl-2-(4-(trifluoromethyl)phenyl)-1,2,3,4-tetrahydroquinoline (5j): $0.1057 \mathrm{~g}$ (75\% yield), White solid, m.p. $120-123{ }^{\circ} \mathrm{C} ;[\alpha]_{\mathrm{D}}{ }^{20}=-80.3\left(c 0.69, \mathrm{CH}_{2} \mathrm{Cl}_{2}\right)(87 \%$ ee); IR (film): $3390,1634,1607,1481$ $\mathrm{cm}^{-1} ;{ }^{1} \mathrm{H} \mathrm{NMR}\left(400 \mathrm{MHz}, \mathrm{CDCl}_{3}, \mathrm{ppm}\right) \delta 7.60(\mathrm{~d}, J=8.8 \mathrm{~Hz}, 2 \mathrm{H}), 7.56(\mathrm{~d}, J=8.8 \mathrm{~Hz}, 2 \mathrm{H}), 7.32-7.28(\mathrm{~m}$, 2H), 7.24-7.21 (m, 3H), $7.02(\mathrm{dd}, J=7.6,7.6 \mathrm{~Hz}, 1 \mathrm{H}), 6.65(\mathrm{~d}, J=7.6 \mathrm{~Hz}, 1 \mathrm{H}), 6.60-6.57(\mathrm{~m}, 2 \mathrm{H}), 4.66$ $(\mathrm{dd}, J=11.2,2.8 \mathrm{~Hz}, 1 \mathrm{H}), 4.31(\mathrm{dd}, J=12.0,5.6 \mathrm{~Hz}, 1 \mathrm{H}), 4.04(\mathrm{~s}, 1 \mathrm{H}), 2.31-2.26(\mathrm{~m}, 1 \mathrm{H}), 2.19(\mathrm{~m}, 1 \mathrm{H})$; ${ }^{13} \mathrm{C} \mathrm{NMR}\left(100 \mathrm{MHz}, \mathrm{CDCl}_{3}, \mathrm{ppm}\right) \delta 148.2,145.2,145.1,130.2\left(\mathrm{q}, J_{\mathrm{C}-\mathrm{F}}=32.2 \mathrm{~Hz}\right), 129.9,128.9,128.8$, 127.6, 127.2, 126.8, $125.8\left(\mathrm{q}, J_{\mathrm{C}-\mathrm{F}}=3.7 \mathrm{~Hz}\right), 124.9,124.3\left(\mathrm{q}, J_{\mathrm{C}-\mathrm{F}}=270.4 \mathrm{~Hz}\right), 118.3,114.7,57.2,45.0$, 42.3; ${ }^{19} \mathrm{~F}$ NMR (471 MHz, $\mathrm{CDCl}_{3}$, ppm) $\delta$-62.5; HRMS (ESI) calcd for $\mathrm{C}_{22} \mathrm{H}_{19} \mathrm{NF}_{3}(\mathrm{M}+\mathrm{H}): 354.1464$, Found: 354.1461. 
<smiles>Cc1ccc(C2CC(c3ccccc3)c3ccccc3N2)cc1</smiles>

(-)-cis-4-phenyl-2-(p-tolyl)-1,2,3,4-tetrahydroquinoline (5k): 0.1132 g (95\% yield), White solid, m.p. 93-95 ${ }^{\circ} \mathrm{C} ;[\alpha]_{\mathrm{D}}{ }^{20}=-76.8\left(c 0.63, \mathrm{CH}_{2} \mathrm{Cl}_{2}\right)\left(95 \%\right.$ ee); IR (film): $3383,1605,1514,1480 \mathrm{~cm}^{-1} ;{ }^{1} \mathrm{H}$ NMR (400 $\left.\mathrm{MHz}, \mathrm{CDCl}_{3}, \mathrm{ppm}\right) \delta 7.32(\mathrm{~d}, J=8.0 \mathrm{~Hz}, 2 \mathrm{H}), 7.28(\mathrm{~d}, J=8.0 \mathrm{~Hz}, 2 \mathrm{H}), 7.23-7.18(\mathrm{~m}, 3 \mathrm{H}), 7.16-7.13(\mathrm{~m}$, 2H), $6.98(\mathrm{dd}, J=7.6,7.6 \mathrm{~Hz}, 1 \mathrm{H}), 6.62(\mathrm{~d}, J=7.6 \mathrm{~Hz}, 1 \mathrm{H}), 6.53(\mathrm{dd}, J=8.0,8.0 \mathrm{~Hz}, 2 \mathrm{H}), 4.54(\mathrm{dd}, J=$ 10.8, 3.2 Hz, 1H), 4.27 (dd, $J=11.6,6.0 \mathrm{~Hz}, 1 \mathrm{H}), 3.98(\mathrm{~s}, 1 \mathrm{H}), 2.32(\mathrm{~s}, 3 \mathrm{H}), 2.28-2.14(\mathrm{~m}, 2 \mathrm{H}) ;{ }^{13} \mathrm{C}$ NMR $\left(100 \mathrm{MHz}, \mathrm{CDCl}_{3}, \mathrm{ppm}\right) \delta 145.59,145.56,141.1,137.6,129.8,129.5,128.9,128.7,127.4,126.7,126.6$, 124.9, 117.7, 114.4, 57.2, 45.2, 42.2, 21.3; HRMS (ESI) calcd for $\mathrm{C}_{22} \mathrm{H}_{22} \mathrm{~N}(\mathrm{M}+\mathrm{H})$ : 300.1747, Found: 300.1744 .<smiles>c1ccc(-c2ccc(C3CC(c4ccccc4)c4ccccc4N3)cc2)cc1</smiles>

(-)-cis-2-([1,1'-biphenyl]-4-yl)-4-phenyl-1,2,3,4-tetrahydroquinoline (5l): 0.1359 g (94\% yield), White solid, m.p. $141-144{ }^{\circ} \mathrm{C} ;[\alpha]_{\mathrm{D}}{ }^{20}=-56.4\left(c 0.69, \mathrm{CH}_{2} \mathrm{Cl}_{2}\right)\left(94 \%\right.$ ee); IR (film): 3389, 1603, 1585, $1481 \mathrm{~cm}^{-1}$; ${ }^{1} \mathrm{H}$ NMR $\left(400 \mathrm{MHz}, \mathrm{CDCl}_{3}, \mathrm{ppm}\right) \delta 7.56(\mathrm{~d}, J=8.0 \mathrm{~Hz}, 4 \mathrm{H}), 7.49(\mathrm{~d}, J=8.4 \mathrm{~Hz}, 2 \mathrm{H}), 7.41(\mathrm{dd}, J=7.6$, $7.6 \mathrm{~Hz}, 2 \mathrm{H}), 7.33-7.27(\mathrm{~m}, 3 \mathrm{H}), 7.24-7.19(\mathrm{~m}, 3 \mathrm{H}), 7.00(\mathrm{dd}, J=7.6,7.6 \mathrm{~Hz}, 1 \mathrm{H}), 6.64(\mathrm{~d}, J=7.6 \mathrm{~Hz}$, $1 \mathrm{H}), 6.58-6.54(\mathrm{~m}, 2 \mathrm{H}), 4.61(\mathrm{dd}, J=10.8,2.8 \mathrm{~Hz}, 1 \mathrm{H}), 4.30(\mathrm{dd}, J=12.0,5.6 \mathrm{~Hz}, 1 \mathrm{H}), 4.04(\mathrm{~s}, 1 \mathrm{H})$, 2.33-2.19 (m, 2H); ${ }^{13} \mathrm{C}$ NMR (100 MHz, $\left.\mathrm{CDCl}_{3}, \mathrm{ppm}\right) \delta 145.49,145.46,143.2,141.0,140.9,129.9$, $129.0,128.9,128.7,127.6,127.5,127.4,127.3,127.2,126.7,124.9,117.8,114.5,57.1,45.1,42.3$; HRMS (ESI) calcd for $\mathrm{C}_{27} \mathrm{H}_{24} \mathrm{~N}(\mathrm{M}+\mathrm{H}): 362.1903$, Found: 362.1899. 
<smiles>Cc1ccc(C2CC(c3ccccc3)c3ccccc3N2)cc1C</smiles>

(-)-cis-2-(3-methoxyphenyl)-4-phenyl-1,2,3,4-tetrahydroquinoline (5m): $0.1113 \mathrm{~g}$ (89\% yield), White solid, m.p. $78-81^{\circ} \mathrm{C} ;[\alpha]_{\mathrm{D}}{ }^{20}=-61.4\left(c 0.59, \mathrm{CH}_{2} \mathrm{Cl}_{2}\right)\left(94 \%\right.$ ee); IR (film): $3378,1606,1585,1479 \mathrm{~cm}^{-1} ;{ }^{1} \mathrm{H}$ NMR (400 MHz, $\left.\mathrm{CDCl}_{3}, \mathrm{ppm}\right) \delta$ 7.31-7.28 (m, 2H), 7.24-7.19 (m, 4H), 7.17-7.15 (m, 1H), $7.10(\mathrm{~d}, J=$ $8.0 \mathrm{~Hz}, 1 \mathrm{H}), 6.99(\mathrm{dd}, J=7.6,7.6 \mathrm{~Hz}, 1 \mathrm{H}), 6.62(\mathrm{~d}, J=8.0 \mathrm{~Hz}, 1 \mathrm{H}), 6.56-6.52(\mathrm{~m}, 2 \mathrm{H}), 4.53(\mathrm{dd}, J=10.4$, $3.2 \mathrm{~Hz}, 1 \mathrm{H}), 4.28(\mathrm{dd}, J=11.6,6.0 \mathrm{~Hz}, 1 \mathrm{H}), 4.00(\mathrm{~s}, 1 \mathrm{H}), 2.26(\mathrm{~s}, 3 \mathrm{H}), 2.24(\mathrm{~s}, 3 \mathrm{H}), 2.26-2.19(\mathrm{~m}, 2 \mathrm{H})$; ${ }^{13} \mathrm{C}$ NMR $\left(100 \mathrm{MHz}, \mathrm{CDCl}_{3}, \mathrm{ppm}\right) \delta 145.64,145.61,141.6,137.1,136.2,130.0,129.9,128.9,128.7$, 128.0, 127.4, 126.6, 124.9, 124.2, 117.7, 114.4, 57.2, 45.2, 42.2, 20.0, 19.6; HRMS (ESI) calcd for $\mathrm{C}_{23} \mathrm{H}_{24} \mathrm{~N}(\mathrm{M}+\mathrm{H}):$ 314.1903, Found: 314.1901 .<smiles>c1ccc(C2CC(c3ccc4ccccc4c3)Nc3ccccc32)cc1</smiles>

(-)-cis-2-(naphthalen-2-yl)-4-phenyl-1,2,3,4-tetrahydroquinoline (5n): 0.1246 g (90\% yield), White solid, m.p. $86-89^{\circ} \mathrm{C} ;[\alpha]_{\mathrm{D}}{ }^{20}=-83.2\left(c 0.72, \mathrm{CH}_{2} \mathrm{Cl}_{2}\right)\left(96 \%\right.$ ee); IR (film): $3381,1604,1586,1479 \mathrm{~cm}^{-1} ;{ }^{1} \mathrm{H}$ NMR $\left(400 \mathrm{MHz}, \mathrm{CDCl}_{3}, \mathrm{ppm}\right) \delta 7.88(\mathrm{~s}, 1 \mathrm{H}), 7.82-7.80(\mathrm{~m}, 3 \mathrm{H}), 7.55(\mathrm{dd}, J=8.4,1.6 \mathrm{~Hz}, 1 \mathrm{H})$, 7.48-7.42 (m, 2H), 7.32-7.28 (m, 2H), 7.26-7.19 (m, 3H), 7.02 (dd, J = 7.6, 7.6 Hz, 1H), $6.66(\mathrm{~d}, J=7.6$ $\mathrm{Hz}, 1 \mathrm{H}), 6.58(\mathrm{dd}, J=7.6,7.6 \mathrm{~Hz}, 2 \mathrm{H}), 4.74(\mathrm{dd}, J=10.4,3.2 \mathrm{~Hz}, 1 \mathrm{H}), 4.33(\mathrm{dd}, J=11.6,6.0 \mathrm{~Hz}, 1 \mathrm{H})$, $4.12(\mathrm{~s}, 1 \mathrm{H}), 2.36-2.24(\mathrm{~m}, 2 \mathrm{H}) ;{ }^{13} \mathrm{C} \mathrm{NMR}\left(100 \mathrm{MHz}, \mathrm{CDCl}_{3}, \mathrm{ppm}\right) \delta 145.50,145.47,141.5,133.7,133.3$, $129.9,128.9,128.8,128.6,128.0,127.9,127.5,126.7,126.4,126.0,125.4,125.04,125.01,117.9,114.5$, 57.6, 45.2, 42.2; HRMS (ESI) calcd for $\mathrm{C}_{25} \mathrm{H}_{22} \mathrm{~N}(\mathrm{M}+\mathrm{H})$ : 336.1747, Found: 336.1742 .<smiles>c1ccc(C2CC(c3ccco3)Nc3ccccc32)cc1</smiles> 
(-)-cis-2-(furan-2-yl)-4-phenyl-1,2,3,4-tetrahydroquinoline (5o): 0.0881 g (80\% yield), White solid, m.p. $125-128^{\circ} \mathrm{C} ;[\alpha]_{\mathrm{D}}{ }^{20}=-52.5\left(c 0.60, \mathrm{CH}_{2} \mathrm{Cl}_{2}\right)\left(87 \%\right.$ ee); IR (film): 3391, 1606, 1585, $1480 \mathrm{~cm}^{-1} ;{ }^{1} \mathrm{H}$ NMR (400 MHz, $\left.\mathrm{CDCl}_{3}, \mathrm{ppm}\right) \delta 7.36(\mathrm{~d}, J=1.2 \mathrm{~Hz}, 1 \mathrm{H}), 7.33-7.29(\mathrm{~m}, 2 \mathrm{H}), 7.25-7.22(\mathrm{~m}, 3 \mathrm{H}), 6.99$ (dd, $J=7.6,7.6 \mathrm{~Hz}, 1 \mathrm{H}), 6.63(\mathrm{~d}, J=7.2 \mathrm{~Hz}, 1 \mathrm{H}), 6.57(\mathrm{~d}, J=8.0,7.6 \mathrm{~Hz}, 2 \mathrm{H}), 6.33(\mathrm{dd}, J=3.2,2.0 \mathrm{~Hz}, 1 \mathrm{H})$, $6.25(\mathrm{~d}, J=3.2 \mathrm{~Hz}, 1 \mathrm{H}), 4.69(\mathrm{dd}, J=11.2,2.4 \mathrm{~Hz}, 1 \mathrm{H}), 4.25(\mathrm{dd}, J=12.4,5.6 \mathrm{~Hz}, 1 \mathrm{H}), 4.16(\mathrm{~s}, 1 \mathrm{H})$, 2.46-2.41 (m, 1H), $2.31(\mathrm{~m}, 1 \mathrm{H}) ;{ }^{13} \mathrm{C} \mathrm{NMR}\left(100 \mathrm{MHz}, \mathrm{CDCl}_{3}, \mathrm{ppm}\right) \delta 156.3,145.4,144.7,142.0,129.9$, $128.9,128.8,127.5,126.8,125.0,118.2,114.9,110.4,105.3,50.6,44.4,38.1$; HRMS (ESI) calcd for $\mathrm{C}_{19} \mathrm{H}_{18} \mathrm{NO}(\mathrm{M}+\mathrm{H}): 276.1383$, Found: 276.1382 .

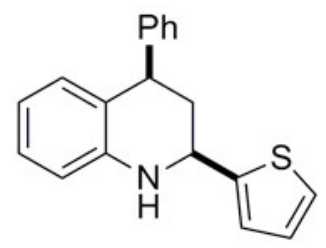

(-)-cis-4-phenyl-2-(thiophen-2-yl)-1,2,3,4-tetrahydroquinoline (5p): $0.1071 \mathrm{~g}$ (93\% yield), White solid, m.p. $157-158{ }^{\circ} \mathrm{C} ;[\alpha]_{\mathrm{D}}{ }^{20}=-41.6\left(c 0.63, \mathrm{CH}_{2} \mathrm{Cl}_{2}\right)\left(89 \%\right.$ ee); IR (film): $3369,1603,1476,1312 \mathrm{~cm}^{-1} ;{ }^{1} \mathrm{H}$ NMR (400 MHz, $\left.\mathrm{CDCl}_{3}, \mathrm{ppm}\right)$ 8 7.33-7.29 (m, 2H), 7.25-7.21 (m, 4H), $7.06(\mathrm{~d}, J=3.2 \mathrm{~Hz}, 1 \mathrm{H}), 7.00$ (dd, $J=7.6,7.6 \mathrm{~Hz}, 1 \mathrm{H}), 6.97-6.95(\mathrm{~m}, 1 \mathrm{H}), 6.64(\mathrm{~d}, J=7.6 \mathrm{~Hz}, 1 \mathrm{H}), 6.59-6.56(\mathrm{~m}, 2 \mathrm{H}), 4.92(\mathrm{dd}, J=11.2$, $2.4 \mathrm{~Hz}, 1 \mathrm{H}), 4.30(\mathrm{dd}, J=12.4,5.6 \mathrm{~Hz}, 1 \mathrm{H}), 4.18(\mathrm{~s}, 1 \mathrm{H}), 2.42-2.38(\mathrm{~m}, 1 \mathrm{H}), 2.29(\mathrm{~m}, 1 \mathrm{H}) ;{ }^{13} \mathrm{C} \mathrm{NMR}$ (100 MHz, $\left.\mathrm{CDCl}_{3}, \mathrm{ppm}\right) \delta 148.0,145.3,145.0,129.9,128.9,128.8,127.5,126.80,126.78,125.0,124.4$, 124.0, 118.3, 114.7, 53.1, 45.0, 43.2; HRMS (ESI) calcd for $\mathrm{C}_{19} \mathrm{H}_{18} \mathrm{NS}(\mathrm{M}+\mathrm{H})$ : 292.1155, Found: 292.1151.<smiles>c1ccc(C2CC(c3ccsc3)Nc3ccccc32)cc1</smiles>

(-)-cis-4-phenyl-2-(thiophen-3-yl)-1,2,3,4-tetrahydroquinoline (5q): $0.1025 \mathrm{~g}$ (88\% yield), White solid, m.p. $126-129^{\circ} \mathrm{C} ;[\alpha]_{\mathrm{D}}{ }^{20}=-79.2\left(c 0.70, \mathrm{CH}_{2} \mathrm{Cl}_{2}\right)\left(90 \%\right.$ ee); IR (film): $3385,1605,1583,1488 \mathrm{~cm}^{-1} ;{ }^{1} \mathrm{H}$ NMR (400 MHz, $\left.\mathrm{CDCl}_{3}, \mathrm{ppm}\right) \delta$ 7.32-7.27 (m, 3H), 7.25-7.20 (m, 4H), 7.14-7.12 (m, 1H), $6.99(\mathrm{dd}, J=$ 
7.6, 7.6 Hz, 1H), $6.63(\mathrm{~d}, J=8.0 \mathrm{~Hz}, 1 \mathrm{H}), 6.56(\mathrm{~d}, J=7.6,6.8 \mathrm{~Hz}, 2 \mathrm{H}), 4.72(\mathrm{dd}, J=10.8,2.4 \mathrm{~Hz}, 1 \mathrm{H})$, $4.27(\mathrm{dd}, J=12.0,5.6 \mathrm{~Hz}, 1 \mathrm{H}), 4.06(\mathrm{~s}, 1 \mathrm{H}), 2.35-2.29(\mathrm{~m}, 1 \mathrm{H}), 2.21(\mathrm{~m}, 1 \mathrm{H}) ;{ }^{13} \mathrm{C} \mathrm{NMR}(100 \mathrm{MHz}$, $\left.\mathrm{CDCl}_{3}, \mathrm{ppm}\right) \delta 145.5,145.32,145.26,129.9,128.9,128.7,127.4,126.7,126.4,126.3,125.0,121.0,118.0$ 114.5, 53.1, 44.9, 41.6; HRMS (ESI) calcd for $\mathrm{C}_{19} \mathrm{H}_{18} \mathrm{NS}(\mathrm{M}+\mathrm{H})$ : 292.1155, Found: 292.1151.<smiles>Fc1ccc(C2CC(c3ccccc3)Nc3ccccc32)cc1</smiles>

(-)-cis-6-chloro-2,4-diphenyl-1,2,3,4-tetrahydroquinoline (5r): 0.1081 g (89\% yield), White solid, m.p. $109-118^{\circ} \mathrm{C} ;[\alpha]_{\mathrm{D}}{ }^{20}=-106.1\left(c 0.74, \mathrm{CH}_{2} \mathrm{Cl}_{2}\right)\left(96 \%\right.$ ee); IR (film): $3391,1606,1508,1480 \mathrm{~cm}^{-1} ;{ }^{1} \mathrm{H}$ NMR $\left(400 \mathrm{MHz}, \mathrm{CDCl}_{3}, \mathrm{ppm}\right) \delta 7.43(\mathrm{~d}, J=7.6 \mathrm{~Hz}, 2 \mathrm{H}), 7.34(\mathrm{dd}, J=7.6,7.2 \mathrm{~Hz}, 2 \mathrm{H}), 7.29-7.27(\mathrm{~m}, 1 \mathrm{H})$, 7.20-7.16 (m, 2H), 7.02-6.95 (m, 3H), 6.62-6.59 (m, 1H), 6.58-6.54 (m, 2H), $4.58(\mathrm{dd}, J=11.2,2.4 \mathrm{~Hz}$, $1 \mathrm{H}), 4.29$ (dd, $J=12.0,5.6 \mathrm{~Hz}, 1 \mathrm{H}), 4.03(\mathrm{~s}, 1 \mathrm{H}), 2.27-2.23(\mathrm{~m}, 1 \mathrm{H}), 2.15(\mathrm{~m}, 1 \mathrm{H}) ;{ }^{13} \mathrm{C} \mathrm{NMR}(100 \mathrm{MHz}$, $\left.\mathrm{CDCl}_{3}, \mathrm{ppm}\right) \delta 161.8\left(\mathrm{~d}, J_{\mathrm{C}-\mathrm{F}}=242.7 \mathrm{~Hz}\right), 145.5,144.0,141.2\left(\mathrm{~d}, J_{\mathrm{C}-\mathrm{F}}=3.3 \mathrm{~Hz}\right), 130.2\left(\mathrm{~d}, J_{\mathrm{C}-\mathrm{F}}=7.8 \mathrm{~Hz}\right)$, 129.7, 128.9, 18.0, 127.6, 126.8, 124.7, 117.8, $115.5\left(\mathrm{~d}, J_{\mathrm{C}-\mathrm{F}}=21.0 \mathrm{~Hz}\right) .114 .5,57.4,44.4,42.4 ;{ }^{19} \mathrm{~F}$ NMR (471 MHz, $\left.\mathrm{CDCl}_{3}, \mathrm{ppm}\right) \delta$-116.7; HRMS (ESI) calcd for $\mathrm{C}_{21} \mathrm{H}_{19} \mathrm{NF}(\mathrm{M}+\mathrm{H})$ : 304.1496, Found: 304.1493.<smiles>Clc1ccc(C2CC(c3ccccc3)Nc3ccccc32)cc1</smiles>

(-)-cis-4-(4-chlorophenyl)-2-phenyl-1,2,3,4-tetrahydroquinoline (5s): 0.1240 g (97\% yield), White solid, m.p. $170-173{ }^{\circ} \mathrm{C} ;[\alpha]_{\mathrm{D}}{ }^{20}=-108.2\left(c 0.91, \mathrm{CH}_{2} \mathrm{Cl}_{2}\right)\left(95 \%\right.$ ee); IR (film): $3398,1606,1586,1480 \mathrm{~cm}^{-1}$; ${ }^{1} \mathrm{H}$ NMR $\left(400 \mathrm{MHz}, \mathrm{CDCl}_{3}, \mathrm{ppm}\right) \delta 7.43(\mathrm{~d}, J=7.2 \mathrm{~Hz}, 2 \mathrm{H}), 7.35(\mathrm{dd}, J=7.6,7.2 \mathrm{~Hz}, 2 \mathrm{H}), 7.30-7.25(\mathrm{~m}$, $3 \mathrm{H}), 7.16(\mathrm{~d}, J=8.4 \mathrm{~Hz}, 2 \mathrm{H}), 7.01(\mathrm{dd}, J=7.6,7.6 \mathrm{~Hz}, 1 \mathrm{H}), 6.60-6.55(\mathrm{~m}, 3 \mathrm{H}), 4.57(\mathrm{dd}, J=11.2,2.4 \mathrm{~Hz}$, 
$1 \mathrm{H}), 4.28(\mathrm{dd}, J=12.0,5.6 \mathrm{~Hz}, 1 \mathrm{H}), 4.05(\mathrm{~s}, 1 \mathrm{H}), 2.27-2.23(\mathrm{~m}, 1 \mathrm{H}), 2.15(\mathrm{~m}, 1 \mathrm{H}) ;{ }^{13} \mathrm{C}$ NMR $(100 \mathrm{MHz}$, $\left.\mathrm{CDCl}_{3}, \mathrm{ppm}\right) \delta 145.5,144.1,143.9,132.3,130.2,129.7,128.90,128.88,128.0,127.6,126.8,124.3,117.9$, 114.6, 57.4, 44.5, 42.3; HRMS (ESI) calcd for $\mathrm{C}_{21} \mathrm{H}_{19} \mathrm{NCl}(\mathrm{M}+\mathrm{H})$ : 320.1201, Found: 320.1197 .<smiles>Brc1ccc(C2CC(c3ccccc3)Nc3ccccc32)cc1</smiles>

(-)-cis-4-(4-bromophenyl)-2-phenyl-1,2,3,4-tetrahydroquinoline (5t): $0.1248 \mathrm{~g}$ (86\% yield), White solid, m.p. $159-162{ }^{\circ} \mathrm{C} ;[\alpha]_{\mathrm{D}}{ }^{20}=-167.5\left(c 0.92, \mathrm{CH}_{2} \mathrm{Cl}_{2}\right)\left(94 \%\right.$ ee); IR (film): $3368,1605,1482,1456 \mathrm{~cm}^{-1}$; ${ }^{1} \mathrm{H}$ NMR $\left(400 \mathrm{MHz}, \mathrm{CDCl}_{3}, \mathrm{ppm}\right) \delta$ 7.44-7.40 (m, 4H), $7.34(\mathrm{dd}, J=7.6,7.2 \mathrm{~Hz}, 2 \mathrm{H}), 7.30-7.26(\mathrm{~m}, 1 \mathrm{H})$, $7.11(\mathrm{~d}, J=8.4 \mathrm{~Hz}, 2 \mathrm{H}), 7.00(\mathrm{ddd}, J=7.6,7.6,1.2 \mathrm{~Hz}, 1 \mathrm{H}), 6.60-6.54(\mathrm{~m}, 3 \mathrm{H}), 4.57(\mathrm{dd}, J=11.2,2.4$ $\mathrm{Hz}, 1 \mathrm{H}), 4.27(\mathrm{dd}, J=12.0,5.6 \mathrm{~Hz}, 1 \mathrm{H}), 4.04(\mathrm{~s}, 1 \mathrm{H}), 2.27-2.22(\mathrm{~m}, 1 \mathrm{H}), 2.14(\mathrm{~m}, 1 \mathrm{H}) ;{ }^{13} \mathrm{C} \mathrm{NMR}(100$ $\left.\mathrm{MHz}, \mathrm{CDCl}_{3}, \mathrm{ppm}\right) \delta 145.5,144.6,143.9,131.8,130.6,129.7,128.9,128.0,127.7,126.8,124.2,120.4$, 117.9, 114.6, 57.3, 44.6, 42.2; HRMS (ESI) calcd for $\mathrm{C}_{21} \mathrm{H}_{19} \mathrm{NBr}(\mathrm{M}+\mathrm{H})$ : 364.0695, Found: 364.0690 .<smiles>Cc1ccc(C2CC(c3ccccc3)Nc3ccccc32)cc1</smiles>

(-)-cis-2-phenyl-4-(p-tolyl)-1,2,3,4-tetrahydroquinoline (5u): 0.1076 g, (90\% yield) White solid, m.p. $131-133{ }^{\circ} \mathrm{C} ;[\alpha]_{\mathrm{D}}{ }^{20}=-63.4\left(c 0.91, \mathrm{CH}_{2} \mathrm{Cl}_{2}\right)\left(90 \%\right.$ ee); IR (film): $3379,1605,1513,1479 \mathrm{~cm}^{-1} ;{ }^{1} \mathrm{H}$ NMR $\left(400 \mathrm{MHz}, \mathrm{CDCl}_{3}, \mathrm{ppm}\right) \delta 7.42(\mathrm{~d}, J=7.2 \mathrm{~Hz}, 2 \mathrm{H}), 7.33(\mathrm{dd}, J=7.6,6.8 \mathrm{~Hz}, 2 \mathrm{H}), 7.28-7.24(\mathrm{~m}, 1 \mathrm{H})$, 7.13-7.09 (m, 4H), $6.98(\mathrm{dd}, J=7.6,7.6 \mathrm{~Hz}, 1 \mathrm{H}), 6.64(\mathrm{~d}, J=7.6 \mathrm{~Hz}, 1 \mathrm{H}), 6.54(\mathrm{dd}, J=8.0,7.6 \mathrm{~Hz}, 2 \mathrm{H})$, $4.57(\mathrm{dd}, J=10.8,3.2 \mathrm{~Hz}, 1 \mathrm{H}), 4.25(\mathrm{dd}, J=12.0,6.0 \mathrm{~Hz}, 1 \mathrm{H}), 4.00(\mathrm{~s}, 1 \mathrm{H}), 2.31(\mathrm{~s}, 3 \mathrm{H}), 2.28-2.23(\mathrm{~m}$, 1H), $2.19(\mathrm{~m}, 1 \mathrm{H}) ;{ }^{13} \mathrm{C}$ NMR (100 MHz, $\left.\mathrm{CDCl}_{3}, \mathrm{ppm}\right) \delta 145.5,144.2,142.4,136.1,129.8,129.4,128.8$, 
128.7, 127.9, 127.4, 126.8, 125.1, 117.7, 114.4, 57.5, 44.7, 42.3, 21.3; HRMS (ESI) calcd for $\mathrm{C}_{22} \mathrm{H}_{22} \mathrm{~N}$ $(\mathrm{M}+\mathrm{H}): 300.1747$, Found: 300.1744.<smiles>COc1ccc(C2CC(c3ccccc3)Nc3ccccc32)cc1</smiles>

(-)-cis-4-(4-methoxyphenyl)-2-phenyl-1,2,3,4-tetrahydroquinoline (5v): $0.1161 \mathrm{~g}$ (92\% yield), White solid, m.p. $103-106{ }^{\circ} \mathrm{C} ;[\alpha]_{\mathrm{D}}{ }^{20}=-59.3\left(c 0.72, \mathrm{CH}_{2} \mathrm{Cl}_{2}\right)\left(91 \%\right.$ ee); IR (film): 3377, 1608, 1511, $1479 \mathrm{~cm}^{-1}$; ${ }^{1} \mathrm{H}$ NMR (400 MHz, $\left.\mathrm{CDCl}_{3}, \mathrm{ppm}\right) \delta 7.44(\mathrm{~d}, J=7.2 \mathrm{~Hz}, 2 \mathrm{H}), 7.34(\mathrm{dd}, J=7.6,7.2 \mathrm{~Hz}, 2 \mathrm{H})$, 7.29-7.25 (m, $1 \mathrm{H}), 7.15(\mathrm{~d}, J=8.8 \mathrm{~Hz}, 2 \mathrm{H}), 6.99(\mathrm{dd}, J=7.6,7.6 \mathrm{~Hz}, 1 \mathrm{H}), 6.84(\mathrm{~d}, J=8.8 \mathrm{~Hz}, 2 \mathrm{H}), 6.65(\mathrm{~d}, J=8.0 \mathrm{~Hz}$, $1 \mathrm{H}), 6.56(\mathrm{dd}, J=8.0,6.4 \mathrm{~Hz}, 2 \mathrm{H}), 4.59(\mathrm{dd}, J=10.8,2.8 \mathrm{~Hz}, 1 \mathrm{H}), 4.25(\mathrm{dd}, J=12.0,5.6 \mathrm{~Hz}, 1 \mathrm{H}), 4.04$ (s, 1H), $3.78(\mathrm{~s}, 3 \mathrm{H}), 2.28-2.13(\mathrm{~m}, 2 \mathrm{H}) ;{ }^{13} \mathrm{C} \mathrm{NMR}\left(100 \mathrm{MHz}, \mathrm{CDCl}_{3}, \mathrm{ppm}\right) \delta 158.4,145.5,144.2,137.5$, 129.8, 128.9, 127.9, 127.4, 126.8, 125.3, 117.7, 114.4, 114.1, 57.5, 55.4, 44.3, 42.3; HRMS (ESI) calcd for $\mathrm{C}_{22} \mathrm{H}_{22} \mathrm{ON}(\mathrm{M}+\mathrm{H}): 316.1696$, Found: 316.1692 .<smiles>C1=C(C2CC(c3ccccc3)Nc3ccccc32)CCCC1</smiles>

(-)-cis-4-(cyclohex-1-en-1-yl)-2-phenyl-1,2,3,4-tetrahydroquinoline (5w): $0.1069 \mathrm{~g}$ (93\% yield), White solid, m.p. $70-72{ }^{\circ} \mathrm{C} ;[\alpha]_{\mathrm{D}}{ }^{20}=-75.0\left(c 0.82, \mathrm{CH}_{2} \mathrm{Cl}_{2}\right)\left(86 \%\right.$ ee); IR (film): 3379, 1604, 1582, $1478 \mathrm{~cm}^{-1} ;{ }^{1} \mathrm{H}$ NMR (400 MHz, $\left.\mathrm{CDCl}_{3}, \mathrm{ppm}\right) \delta$ 7.43-7.41 (m, 2H), 7.35 (dd, $\left.J=7.6,7.2 \mathrm{~Hz}, 2 \mathrm{H}\right), 7.30-7.26(\mathrm{~m}, 1 \mathrm{H})$, 7.03-6.97 (m, 2H), $6.66(\mathrm{dd}, J=7.6,7.6 \mathrm{~Hz}, 1 \mathrm{H}), 6.51(\mathrm{~d}, J=8.0 \mathrm{~Hz}, 1 \mathrm{H}), 5.68-5.68(\mathrm{~m}, 1 \mathrm{H}), 4.47(\mathrm{dd}, J$ $=10.8,2.8 \mathrm{~Hz}, 1 \mathrm{H}), 3.93(\mathrm{~s}, 1 \mathrm{H}), 3.71(\mathrm{dd}, J=12.0,6.0 \mathrm{~Hz}, 1 \mathrm{H}), 2.16-1.96(\mathrm{~m}, 4 \mathrm{H}), 1.82-1.75(\mathrm{~m}, 2 \mathrm{H})$, 1.67-1.58 (m, 2H), 1.55-1.50 (m, 2H); ${ }^{13} \mathrm{C} \mathrm{NMR}\left(100 \mathrm{MHz}, \mathrm{CDCl}_{3}, \mathrm{ppm}\right) \delta 145.8,144.6,139.3,128.8$, 
$128.5,127.8,127.2,126.8,125.4,123.4,117.8,114.5,57.2,47.2,38.0,25.7,24.4,23.2,22.9$; HRMS (ESI) calcd for $\mathrm{C}_{21} \mathrm{H}_{24} \mathrm{~N}(\mathrm{M}+\mathrm{H})$ : 290.1903, Found: 290.1901.<smiles>Cc1ccc2c(c1)C(c1ccccc1)CC(c1ccccc1)N2</smiles>

(-)-cis-6-methyl-2,4-diphenyl-1,2,3,4-tetrahydroquinoline (5x): 0.1119 g (94\% yield), White solid, m.p. $130-133{ }^{\circ} \mathrm{C} ;[\alpha]_{\mathrm{D}}{ }^{20}=-53.6\left(c 0.76, \mathrm{CH}_{2} \mathrm{Cl}_{2}\right)\left(98 \%\right.$ ee); IR (film): $3375,1615,1506,1454 \mathrm{~cm}^{-1} ;{ }^{1} \mathrm{H}$ NMR $\left(400 \mathrm{MHz}, \mathrm{CDCl}_{3}, \mathrm{ppm}\right) \delta 7.44(\mathrm{~d}, J=7.2 \mathrm{~Hz}, 2 \mathrm{H}), 7.36-7.27(\mathrm{~m}, 5 \mathrm{H}), 7.25-7.21(\mathrm{~m}, 3 \mathrm{H}), 6.82(\mathrm{~d}, J=$ $8.0 \mathrm{~Hz}, 1 \mathrm{H}), 6.50(\mathrm{~d}, J=8.0 \mathrm{~Hz}, 1 \mathrm{H}), 6.47(\mathrm{~s}, 1 \mathrm{H}), 4.55(\mathrm{dd}, J=11.2,2.4 \mathrm{~Hz}, 1 \mathrm{H}), 4.28(\mathrm{dd}, J=12.0,5.6$ $\mathrm{Hz}, 1 \mathrm{H}), 3.94(\mathrm{~s}, 1 \mathrm{H}), 2.30-2.24(\mathrm{~m}, 1 \mathrm{H}), 2.19(\mathrm{~m}, 1 \mathrm{H}), 2.09(\mathrm{~s}, 3 \mathrm{H}) ;{ }^{13} \mathrm{C} \mathrm{NMR}\left(100 \mathrm{MHz}, \mathrm{CDCl}_{3}, \mathrm{ppm}\right) \delta$ $145.8,144.3,143.3,130.3,128.9,128.8,128.7,128.1,127.9,127.0,126.8,126.6,124.9,114.7,57.6,45.2$, 42.7, 20.7; HRMS (ESI) calcd for $\mathrm{C}_{22} \mathrm{H}_{22} \mathrm{~N}(\mathrm{M}+\mathrm{H})$ : 300.1747, Found: 300.1744 .<smiles>Clc1ccc2c(c1)C(c1ccccc1)CC(c1ccccc1)N2</smiles>

(-)-cis-6-chloro-2,4-diphenyl-1,2,3,4-tetrahydroquinoline (5y): 0.1065 g (84\% yield), White solid, m.p. $106-108^{\circ} \mathrm{C} ;[\alpha]_{\mathrm{D}}{ }^{20}=-40.0\left(c 0.53, \mathrm{CH}_{2} \mathrm{Cl}_{2}\right)(94 \%$ ee $)$; IR (film): $3370,1505,1488,1470 \mathrm{~cm}^{-1} ;{ }^{1} \mathrm{H}$ NMR $\left(400 \mathrm{MHz}, \mathrm{CDCl}_{3}, \mathrm{ppm}\right) \delta 7.42(\mathrm{~d}, J=7.6 \mathrm{~Hz}, 2 \mathrm{H}), 7.36-7.25(\mathrm{~m}, 5 \mathrm{H}), 7.23-7.20(\mathrm{~m}, 3 \mathrm{H}), 6.94(\mathrm{dd}, J=$ 8.4, $2.4 \mathrm{~Hz}, 1 \mathrm{H}), 6.59(\mathrm{~s}, 1 \mathrm{H}), 6.47(\mathrm{~d}, J=8.4 \mathrm{~Hz}, 1 \mathrm{H}), 4.54(\mathrm{dd}, J=11.2,2.8 \mathrm{~Hz}, 1 \mathrm{H}), 4.23(\mathrm{dd}, J=12.0$, $5.6 \mathrm{~Hz}, 1 \mathrm{H}), 4.04(\mathrm{~s}, 1 \mathrm{H}), 2.28-2.24(\mathrm{~m}, 1 \mathrm{H}), 2.16(\mathrm{~m}, 1 \mathrm{H}) ;{ }^{13} \mathrm{C} \mathrm{NMR}\left(100 \mathrm{MHz}, \mathrm{CDCl}_{3}, \mathrm{ppm}\right) \delta$ 144.6, $144.1,143.7,129.4,128.9,128.8,128.1,127.3,127.0,126.8,126.5,122.2,115.5,57.4,45.1,41.8$; HRMS (ESI) calcd for $\mathrm{C}_{21} \mathrm{H}_{19} \mathrm{NCl}(\mathrm{M}+\mathrm{H})$ : 320.1201, Found: 320.1198 .<smiles>Cc1cc2ccccc2nc1-c1ccccc1</smiles> 
3-methyl-2-phenylquinoline (6a): $1.62 \mathrm{~g}\left(74 \%\right.$ yield), ${ }^{1} \mathrm{H} \mathrm{NMR}\left(400 \mathrm{MHz}, \mathrm{CDCl}_{3}, \mathrm{ppm}\right) \delta 8.13(\mathrm{~d}, J=$ $8.4 \mathrm{~Hz}, 1 \mathrm{H}), 7.97(\mathrm{~s}, 1 \mathrm{H}), 7.74(\mathrm{~d}, J=8.0 \mathrm{~Hz}, 1 \mathrm{H}), 7.64(\mathrm{dd}, J=7.6,7.6 \mathrm{~Hz}, 1 \mathrm{H}), 7.59-7.57$ (m, 2H), 7.50-7.40 (m, 4H), $2.43(\mathrm{~s}, 3 \mathrm{H}) ;{ }^{13} \mathrm{C} \mathrm{NMR}\left(100 \mathrm{MHz}, \mathrm{CDCl}_{3}, \mathrm{ppm}\right) \delta 160.7,146.8,141.0,136.8,129.5$, $129.3,129.0,128.9,128.4,128.3,127.7,126.8,126.5,20.8$.

Martínez, R.; Ramón, J. D.; Yus, M. J. Org. Chem. 2008, 73, 9778.<smiles>Cc1cc2ccccc2nc1-c1cccc(Cl)c1</smiles>

2-(3-chlorophenyl)-3-methylquinoline (6b): 1.70 g (67\% yield), White solid, m.p. 81-83 ${ }^{\circ} \mathrm{C}$; IR (film): $1620,1594,1557,1492 \mathrm{~cm}^{-1} ;{ }^{1} \mathrm{H}$ NMR $\left(400 \mathrm{MHz}, \mathrm{CDCl}_{3}, \mathrm{ppm}\right) \delta 8.11(\mathrm{~d}, J=8.4 \mathrm{~Hz}, 1 \mathrm{H}), 8.01(\mathrm{~s}, 1 \mathrm{H})$, $7.77(\mathrm{~d}, J=8.0 \mathrm{~Hz}, 1 \mathrm{H}), 7.67(\mathrm{ddd}, J=7.6,7.6,0.8 \mathrm{~Hz}, 1 \mathrm{H}), 7.60(\mathrm{~d}, J=0.8 \mathrm{~Hz}, 1 \mathrm{H}), 7.52(\mathrm{dd}, J=8.0$ $\mathrm{Hz}, 1 \mathrm{H}), 7.48-7.44(\mathrm{~m}, 1 \mathrm{H}), 7.43-7.41(\mathrm{~m}, 2 \mathrm{H}), 2.45(\mathrm{~s}, 3 \mathrm{H}) ;{ }^{13} \mathrm{C} \mathrm{NMR}\left(100 \mathrm{MHz}, \mathrm{CDCl}_{3}, \mathrm{ppm}\right) \delta 159.1$, $146.8,142.8,137.2,134.5,129.8,129.5,129.3,129.2,129.1,128.5,127.9,127.3,126.94,126.91,20.7$; HRMS (ESI) calcd for $\mathrm{C}_{16} \mathrm{H}_{13} \mathrm{NCl}(\mathrm{M}+\mathrm{H})$ : 254.0731, Found: 254.0729.<smiles>Cc1cc2ccccc2nc1-c1cccc(Br)c1</smiles>

2-(3-bromophenyl)-3-methylquinoline (6c): 1.73 g (58\% yield), White solid, m.p. $115-117{ }^{\circ} \mathrm{C}$; IR (film): 1618, 1593, 1560, $1492 \mathrm{~cm}^{-1} ;{ }^{1} \mathrm{H}$ NMR (400 MHz, $\left.\mathrm{CDCl}_{3}, \mathrm{ppm}\right) \delta 8.11(\mathrm{~d}, J=8.4 \mathrm{~Hz}, 1 \mathrm{H}), 8.03$ (s, 1H), $7.79(\mathrm{~d}, J=8.0 \mathrm{~Hz}, 1 \mathrm{H}), 7.76(\mathrm{dd}, J=1.6,1.6 \mathrm{~Hz}, 1 \mathrm{H}), 7.67(\mathrm{ddd}, J=8.4,8.4,1.2 \mathrm{~Hz}, 1 \mathrm{H})$, 7.59-7.56 (m, 1H), 7.56-7.51 (m, 2H), $7.36(\mathrm{dd}, J=8.0,8.0 \mathrm{~Hz}, 1 \mathrm{H}), 2.46(\mathrm{~s}, 3 \mathrm{H}) ;{ }^{13} \mathrm{C} \mathrm{NMR}(100 \mathrm{MHz}$, $\left.\mathrm{CDCl}_{3}, \mathrm{ppm}\right) \delta 159.0,146.8,143.1,137.2,132.2,131.5,130.0,129.5,129.2,129.1,127.9,127.7,127.0$ 126.9, 122.7, 20.7; HRMS (ESI) calcd for $\mathrm{C}_{16} \mathrm{H}_{13} \mathrm{NBr}(\mathrm{M}+\mathrm{H})$ : 298.0226, Found: 298.0224.<smiles>Cc1cc2ccccc2nc1-c1cccc(C(F)(F)F)c1</smiles> 
3-methyl-2-(3-(trifluoromethyl)phenyl)quinoline (6d): $1.61 \mathrm{~g}\left(56 \%\right.$ yield), ${ }^{1} \mathrm{H} \mathrm{NMR}\left(400 \mathrm{MHz}, \mathrm{CDCl}_{3}\right.$, ppm) $\delta 8.11(\mathrm{~d}, J=8.4 \mathrm{~Hz}, 1 \mathrm{H}), 7.99(\mathrm{~s}, 1 \mathrm{H}), 7.95(\mathrm{~s}, 1 \mathrm{H}), 7.76(\mathrm{dd}, J=8.0,8.0 \mathrm{~Hz}, 1 \mathrm{H}), 7.66(\mathrm{dd}, J=$ 7.6, 1.2 Hz, 1H), $7.68(\mathrm{~d}, J=8.4 \mathrm{~Hz}, 1 \mathrm{H}), 7.65(\mathrm{~d}, J=7.6 \mathrm{~Hz}, 1 \mathrm{H}), 7.58(\mathrm{dd}, J=7.6,7.6 \mathrm{~Hz}, 1 \mathrm{H}), 7.50$ $(\mathrm{dd}, J=7.6,7.6 \mathrm{~Hz}, 1 \mathrm{H}), 2.42(\mathrm{~s}, 3 \mathrm{H}) ;{ }^{13} \mathrm{C} \mathrm{NMR}\left(100 \mathrm{MHz}, \mathrm{CDCl}_{3}, \mathrm{ppm}\right) \delta 158.9,146.8,141.7,137.3$, $132.4,130.9\left(\mathrm{q}, J_{\mathrm{C}-\mathrm{F}}=32.1 \mathrm{~Hz}\right), 129.4,129.2,129.0,128.9,127.9,127.0,126.9,126.0\left(\mathrm{q}, J_{\mathrm{C}-\mathrm{F}}=3.7 \mathrm{~Hz}\right)$, $125.1\left(\mathrm{q}, J_{\mathrm{C}-\mathrm{F}}=3.7 \mathrm{~Hz}\right), 124.3\left(\mathrm{q}, J_{\mathrm{C}-\mathrm{F}}=270.7 \mathrm{~Hz}\right), 20.5$.

Chen, S.-J.; Lu, G.-P.; Cai, C. Synthesis 2015, 47, 976.<smiles>Cc1cccc(-c2nc3ccccc3cc2C)c1</smiles>

3-methyl-2-(m-tolyl)quinoline (6e): 1.65 g (71\% yield), White solid, m.p. $41-43{ }^{\circ} \mathrm{C}$; IR (film): 1621 , 1597, 1557, $1489 \mathrm{~cm}^{-1} ;{ }^{1} \mathrm{H}$ NMR (400 MHz, $\left.\mathrm{CDCl}_{3}, \mathrm{ppm}\right) \delta 8.14(\mathrm{~d}, J=8.0 \mathrm{~Hz}, 1 \mathrm{H}), 7.97(\mathrm{~s}, 1 \mathrm{H}), 7.75(\mathrm{~d}$, $J=8.0 \mathrm{~Hz}, 1 \mathrm{H}), 7.64(\mathrm{dd}, J=7.6,7.6 \mathrm{~Hz}, 1 \mathrm{H}), 7.49(\mathrm{dd}, J=7.6,7.6 \mathrm{~Hz}, 1 \mathrm{H}), 7.40(\mathrm{~s}, 1 \mathrm{H}), 7.36-7.35(\mathrm{~m}$, 2H), $7.24(\mathrm{~m}, 1 \mathrm{H}), 2.44(\mathrm{~s}, 3 \mathrm{H}), 2.43(\mathrm{~s}, 3 \mathrm{H}) ;{ }^{13} \mathrm{C} \mathrm{NMR}\left(100 \mathrm{MHz}, \mathrm{CDCl}_{3}, \mathrm{ppm}\right) \delta 161.0,146.8,141.0$, $138.2,136.8,129.7,129.5,129.4,129.1,128.9,128.3,127.8,126.9,126.5,126.0,21.7,20.8$; HRMS (ESI) calcd for $\mathrm{C}_{17} \mathrm{H}_{16} \mathrm{~N}(\mathrm{M}+\mathrm{H}): 234.1277$, Found: 234.1274 .<smiles>Cc1cc2ccccc2nc1-c1ccc(Cl)cc1</smiles>

2-(4-chlorophenyl)-3-methylquinoline (6f): $1.89 \mathrm{~g}$ (75\% yield), ${ }^{1} \mathrm{H} \mathrm{NMR}\left(400 \mathrm{MHz}, \mathrm{CDCl}_{3}, \mathrm{ppm}\right) \delta$ $8.10(\mathrm{~d}, J=8.4 \mathrm{~Hz}, 1 \mathrm{H}), 7.99(\mathrm{~s}, 1 \mathrm{H}), 7.76(\mathrm{~d}, J=8.0 \mathrm{~Hz}, 1 \mathrm{H}), 7.65$ (ddd, $J=7.6,7.6,1.2 \mathrm{~Hz}, 1 \mathrm{H})$, 7.55-7.49 (m, 3H), 7.46-7.44 (m, 2H), $2.44(\mathrm{~s}, 3 \mathrm{H}) ;{ }^{13} \mathrm{C}$ NMR (100 MHz, $\left.\mathrm{CDCl}_{3}, \mathrm{ppm}\right) \delta 159.4,146.8$, $139.4,137.1,134.5,130.5,129.4,129.1,128.7,127.8,126.9,126.8,20.7$.

Chen, S.-J.; Lu, G.-P.; Cai, C. Synthesis 2015, 47, 976. 
<smiles>Cc1cc2ccccc2nc1-c1ccc(Br)cc1</smiles>

2-(4-bromophenyl)-3-methylquinoline (6g): 1.97 g (66\% yield), White solid, m.p. 105-107 ${ }^{\circ} \mathrm{C}$; IR (film): 1600, 1590, 1485, $1440 \mathrm{~cm}^{-1} ;{ }^{1} \mathrm{H} \mathrm{NMR}\left(400 \mathrm{MHz}, \mathrm{CDCl}_{3}, \mathrm{ppm}\right) \delta 8.10(\mathrm{~d}, J=8.4 \mathrm{~Hz}, 1 \mathrm{H}), 8.01(\mathrm{~s}$, 1H), $7.77(\mathrm{~d}, J=8.0 \mathrm{~Hz}, 1 \mathrm{H}), 7.66(\mathrm{ddd}, J=7.6,7.6,1.2 \mathrm{~Hz}, 1 \mathrm{H}), 7.62(\mathrm{~d}, J=8.4 \mathrm{~Hz}, 2 \mathrm{H}), 7.52(\mathrm{ddd}, J=$ 8.0, 8.0, $0.8 \mathrm{~Hz}, 1 \mathrm{H}), 7.48(\mathrm{~d}, J=8.4 \mathrm{~Hz}, 2 \mathrm{H}), 2.45(\mathrm{~s}, 3 \mathrm{H}) ;{ }^{13} \mathrm{C} \mathrm{NMR}\left(100 \mathrm{MHz}, \mathrm{CDCl}_{3}, \mathrm{ppm}\right) \delta 159.4$, $146.9,140.0,137.2,131.7,130.8,129.5,129.12,129.08,127.8,126.9,126.8,122.8,20.7$; HRMS (ESI) calcd for $\mathrm{C}_{16} \mathrm{H}_{13} \mathrm{NBr}(\mathrm{M}+\mathrm{H})$ : 298.0226, Found: 298.0224.<smiles>Cc1cc2ccccc2nc1-c1ccc(C(F)(F)F)cc1</smiles>

3-methyl-2-(4-(trifluoromethyl)phenyl)quinoline (6h): 1.49 g (52\% yield), White solid, m.p. 133-135 ${ }^{\circ} \mathrm{C}$; IR (film): 1590, 1405, 1331, $1162 \mathrm{~cm}^{-1} ;{ }^{1} \mathrm{H}$ NMR (400 MHz, $\left.\mathrm{CDCl}_{3}, \mathrm{ppm}\right) \delta 8.11(\mathrm{~d}, J=8.4 \mathrm{~Hz}, 1 \mathrm{H})$, $8.04(\mathrm{~s}, 1 \mathrm{H}), 7.79(\mathrm{~d}, J=8.0 \mathrm{~Hz}, 1 \mathrm{H}), 7.76-7.74(\mathrm{~m}, 2 \mathrm{H}), 7.72-7.66(\mathrm{~m}, 3 \mathrm{H}), 7.54(\mathrm{dd}, J=7.2,7.2 \mathrm{~Hz}$, 1H), $7.50(\mathrm{dd}, J=7.6,7.6 \mathrm{~Hz}, 1 \mathrm{H}), 2.45(\mathrm{~s}, 3 \mathrm{H}) ;{ }^{13} \mathrm{C} \mathrm{NMR}\left(100 \mathrm{MHz}, \mathrm{CDCl}_{3}, \mathrm{ppm}\right) \delta 159.1,146.8,144.6$, $137.3,130.5\left(\mathrm{q}, J_{\mathrm{C}-\mathrm{F}}=32.2 \mathrm{~Hz}\right), 129.5,129.3,129.0,128.0,127.1,127.0,125.5\left(\mathrm{q}, J_{\mathrm{C}-\mathrm{F}}=3.7 \mathrm{~Hz}\right), 124.4$ $\left(\mathrm{q}, J_{\mathrm{C}-\mathrm{F}}=270.5 \mathrm{~Hz}\right), 20.6 ;{ }^{19} \mathrm{~F} \mathrm{NMR}\left(471 \mathrm{MHz}, \mathrm{CDCl}_{3}, \mathrm{ppm}\right) \delta-62.6 ; \mathrm{HRMS}(\mathrm{ESI})$ calcd for $\mathrm{C}_{17} \mathrm{H}_{13} \mathrm{NF}_{3}$ (M+H): 288.0995, Found: 288.0987.<smiles>Cc1ccc(-c2nc3ccccc3cc2C)cc1</smiles>

3-methyl-2-(p-tolyl)quinoline (6i): $1.86 \mathrm{~g}\left(80 \%\right.$ yield), ${ }^{1} \mathrm{H} \mathrm{NMR}\left(400 \mathrm{MHz}, \mathrm{CDCl}_{3}, \mathrm{ppm}\right) \delta 8.11$ (d, $J=$ $8.4 \mathrm{~Hz}, 1 \mathrm{H}), 7.99(\mathrm{~s}, 1 \mathrm{H}), 7.76(\mathrm{~d}, J=8.0 \mathrm{~Hz}, 1 \mathrm{H}), 7.64(\mathrm{ddd}, J=8.4,8.4,1.2 \mathrm{~Hz}, 1 \mathrm{H}), 7.51-7.48(\mathrm{~m}, 3 \mathrm{H})$, $7.29(\mathrm{~d}, J=8.0 \mathrm{~Hz}, 2 \mathrm{H}), 2.47(\mathrm{~s}, 3 \mathrm{H}), 2.42(\mathrm{~s}, 3 \mathrm{H}) ;{ }^{13} \mathrm{C} \mathrm{NMR}\left(100 \mathrm{MHz}, \mathrm{CDCl}_{3}, \mathrm{ppm}\right) \delta 160.8,146.9$, $138.3,138.2,136.8,129.53,129.48,129.2,129.0,128.8,127.7,126.9,126.5,21.5,20.9$. 
Jacob, J.; Jones, D. W. J. Org. Chem. 2003, 68, 3563.<smiles>COc1ccc(-c2nc3ccccc3cc2C)cc1</smiles>

2-(4-methoxyphenyl)-3-methylquinoline (6j): $1.82 \mathrm{~g}$ (73\% yield), ${ }^{1} \mathrm{H} \mathrm{NMR}\left(400 \mathrm{MHz}, \mathrm{CDCl}_{3}, \mathrm{ppm}\right) \delta$ $8.11(\mathrm{~d}, J=8.4 \mathrm{~Hz}, 1 \mathrm{H}), 7.97(\mathrm{~s}, 1 \mathrm{H}), 7.75(\mathrm{~d}, J=8.0 \mathrm{~Hz}, 1 \mathrm{H}), 7.64(\mathrm{ddd}, J=7.6,7.6,1.2 \mathrm{~Hz}, 1 \mathrm{H}), 7.56$ $(\mathrm{d}, J=8.8 \mathrm{~Hz}, 2 \mathrm{H}), 7.48(\mathrm{dd}, J=7.6,7.6 \mathrm{~Hz}, 1 \mathrm{H}), 7.01(\mathrm{~d}, J=8.8 \mathrm{~Hz}, 2 \mathrm{H}), 3.86(\mathrm{~s}, 3 \mathrm{H}), 2.48(\mathrm{~s}, 3 \mathrm{H}) ;{ }^{13} \mathrm{C}$ NMR (100 MHz, $\left.\mathrm{CDCl}_{3}, \mathrm{ppm}\right) \delta 160.3,159.9,146.9,136.9,133.6,130.5,129.5,129.4,128.8,127.6$, $126.8,126.4,113.9,55.6,21.0$.

Jacob, J.; Jones, D. W. J. Org. Chem. 2003, 68, 3563.<smiles>Cc1cc2ccccc2nc1-c1ccco1</smiles>

2-(furan-2-yl)-3-methylquinoline (6k): $1.01 \mathrm{~g}$ (48\% yield), ${ }^{1} \mathrm{H} \mathrm{NMR}\left(400 \mathrm{MHz}, \mathrm{CDCl}_{3}, \mathrm{ppm}\right) \delta 8.13$ (d, $J=8.4 \mathrm{~Hz}, 1 \mathrm{H}), 7.93(\mathrm{~s}, 1 \mathrm{H}), 7.70-7.62(\mathrm{~m}, 3 \mathrm{H}), 7.46(\mathrm{dd}, J=6.8,6.8 \mathrm{~Hz}, 1 \mathrm{H}), 7.11-7.10(\mathrm{~m}, 1 \mathrm{H})$, 6.59-6.58 (m, 1H), 2.68 (s, 3H); ${ }^{13} \mathrm{C}$ NMR (100 MHz, $\left.\mathrm{CDCl}_{3}, \mathrm{ppm}\right) \delta 153.8,149.1,146.7,143.9,137.8$, 129.4, 129.1, 128.4, 127.4, 126.8, 126.6, 112.6, 111.8. 21.5.

Chen, S.-J.; Lu, G.-P.; Cai, C. Synthesis 2015, 47, 976.<smiles>Cc1cc2ccccc2nc1-c1cccs1</smiles>

3-methyl-2-(thiophen-2-yl)quinoline (6l): $1.14 \mathrm{~g}\left(51 \%\right.$ yield), ${ }^{1} \mathrm{H} \mathrm{NMR}\left(400 \mathrm{MHz}, \mathrm{CDCl}_{3}, \mathrm{ppm}\right) \delta 8.06$ $(\mathrm{d}, J=8.4 \mathrm{~Hz}, 1 \mathrm{H}), 7.90(\mathrm{~s}, 1 \mathrm{H}), 7.67(\mathrm{~d}, J=8.0 \mathrm{~Hz}, 1 \mathrm{H}), 7.61(\mathrm{ddd}, J=7.6,7.6,1.2 \mathrm{~Hz}, 1 \mathrm{H}), 7.57$ (dd, $J$ $=3.6,0.8 \mathrm{~Hz}, 1 \mathrm{H}), 7.46-7.41(\mathrm{~m}, 2 \mathrm{H}), 7.13(\mathrm{dd}, J=5.2,3.6 \mathrm{~Hz}, 1 \mathrm{H}), 2.66(\mathrm{~s}, 3 \mathrm{H}) ;{ }^{13} \mathrm{C} \mathrm{NMR}(100 \mathrm{MHz}$, $\left.\mathrm{CDCl}_{3}, \mathrm{ppm}\right) \delta 152.8,146.7,145.4,137.7,129.2,129.0,128.4,128.3,127.9,127.8,127.3,126.7,126.5$, 22.0. 
Chen, S.-J.; Lu, G.-P.; Cai, C. Synthesis 2015, 47, 976.<smiles>CCc1cc2ccccc2nc1-c1ccccc1</smiles>

3-ethyl-2-phenylquinoline (6m): $1.81 \mathrm{~g}$ (78\% yield), ${ }^{1} \mathrm{H} \mathrm{NMR}\left(400 \mathrm{MHz}, \mathrm{CDCl}_{3}, \mathrm{ppm}\right) \delta 8.13(\mathrm{~d}, J=$ $8.4 \mathrm{~Hz}, 1 \mathrm{H}), 8.03(\mathrm{~s}, 1 \mathrm{H}), 7.80(\mathrm{~d}, J=8.0 \mathrm{~Hz}, 1 \mathrm{H}), 7.65(\mathrm{ddd}, J=7.6,7.6,1.2 \mathrm{~Hz}, 1 \mathrm{H}), 7.56-7.41(\mathrm{~m}, 6 \mathrm{H})$, $2.79(\mathrm{q}, J=7.6 \mathrm{~Hz}, 2 \mathrm{H}), 1.18(\mathrm{t}, J=7.6 \mathrm{~Hz}, 3 \mathrm{H}) ;{ }^{13} \mathrm{C} \mathrm{NMR}\left(100 \mathrm{MHz}, \mathrm{CDCl}_{3}, \mathrm{ppm}\right) \delta 160.8,146.6$, $141.1,135.5,135.1,129.5,129.0,128.9,128.5,128.2,127.9,127.1,126.5,26.2,14.9$.

Martínez, R.; Ramón, J. D.; Yus, M. J. Org. Chem. 2008, 73, 9778.<smiles>CCCc1cc2ccccc2nc1-c1ccccc1</smiles>

2-phenyl-3-propylquinoline (6n): $2.01 \mathrm{~g}$ (81\% yield), ${ }^{1} \mathrm{H} \mathrm{NMR}\left(400 \mathrm{MHz}, \mathrm{CDCl}_{3}, \mathrm{ppm}\right) \delta 8.12(\mathrm{~d}, J=$ $8.4 \mathrm{~Hz}, 1 \mathrm{H}), 8.02(\mathrm{~s}, 1 \mathrm{H}), 7.79(\mathrm{~d}, J=8.0 \mathrm{~Hz}, 1 \mathrm{H}), 7.65(\mathrm{ddd}, J=7.6,7.6,1.2 \mathrm{~Hz}, 1 \mathrm{H}), 7.55-7.41(\mathrm{~m}, 6 \mathrm{H})$, $2.75(\mathrm{t}, J=7.6 \mathrm{~Hz}, 2 \mathrm{H}), 1.61-1.52(\mathrm{~m}, 2 \mathrm{H}), 0.86(\mathrm{t}, J=7.2 \mathrm{~Hz}, 3 \mathrm{H}) ;{ }^{13} \mathrm{C} \mathrm{NMR}\left(100 \mathrm{MHz}, \mathrm{CDCl}_{3}, \mathrm{ppm}\right) \delta$ 161.0, 146.6, 141.2, 135.9, 134.0, 129.5, 129.0, 128.9, 128.5, 128.2, 127.8, 127.1, 126.5, 35.1, 23.9, 14.1. Xi, L.-Y.; Zhang, R.-Y.; Zhang, L.; Chen, S.-Y.; Yu, X.-Q. Org. Biomol. Chem. 2015, 13, 3924.<smiles>CCCCCCc1cc2ccccc2nc1-c1ccccc1</smiles>

3-hexyl-2-phenylquinoline (6o): $2.25 \mathrm{~g}$ (78\% yield), ${ }^{1} \mathrm{H} \mathrm{NMR}\left(400 \mathrm{MHz}, \mathrm{CDCl}_{3}, \mathrm{ppm}\right) \delta 8.12(\mathrm{~d}, J=8.4$ $\mathrm{Hz}, 1 \mathrm{H}), 8.03(\mathrm{~s}, 1 \mathrm{H}), 7.80(\mathrm{~d}, J=7.6 \mathrm{~Hz}, 1 \mathrm{H}), 7.64(\mathrm{ddd}, J=6.8,6.8,1.2 \mathrm{~Hz}, 1 \mathrm{H}), 7.55-7.52(\mathrm{~m}, 3 \mathrm{H})$, 7.50-7.41(m, 3H), $2.76(\mathrm{t}, J=7.6 \mathrm{~Hz}, 2 \mathrm{H}), 1.57-1.49(\mathrm{~m}, 2 \mathrm{H}), 1.27-1.16(\mathrm{~m}, 6 \mathrm{H}), 0.83(\mathrm{t}, J=6.8 \mathrm{~Hz}, 3 \mathrm{H})$; ${ }^{13} \mathrm{C} \mathrm{NMR}\left(100 \mathrm{MHz}, \mathrm{CDCl}_{3}, \mathrm{ppm}\right) \delta 161.0,146.6,141.2,135.9,134.4,129.5,129.0,128.5,128.2,127.9$, $127.1,126.6,33.1,31.7,30.8,29.2,22.7,14.2$.

Lin, X.-F.; Cui, S.-L.; Wang, Y.-G.. Tetrahedron Lett. 2006, 47, 3127. 
$\overbrace{\mathrm{Hh}}^{\mathrm{Me}}$

(+)-cis-3-methyl-2-phenyl-1,2,3,4-tetrahydroquinoline (7a): $0.0812 \mathrm{~g}(91 \%$ yield $),[\alpha]_{\mathrm{D}}{ }^{20}=+21.8(c$ 0.46, $\left.\mathrm{CH}_{2} \mathrm{Cl}_{2}\right)\left(69 \%\right.$ ee) [lit: $[\alpha]_{\mathrm{D}}{ }^{20}=+31.8\left(c 1.1, \mathrm{CHCl}_{3}\right)(82 \%$ ee $\left.)\right] ;{ }^{1} \mathrm{H} \mathrm{NMR}\left(400 \mathrm{MHz}, \mathrm{CDCl}_{3}, \mathrm{ppm}\right) \delta$ 7.33-7.21 (m, 5H), 7.01-6.96 (m, 2H), $6.63(\mathrm{ddd}, J=7.6,7.6,0.8 \mathrm{~Hz}, 1 \mathrm{H}), 6.51(\mathrm{~d}, J=8.0 \mathrm{~Hz}, 1 \mathrm{H}), 4.47$ $(\mathrm{d}, J=3.6 \mathrm{~Hz}, 1 \mathrm{H}), 4.06(\mathrm{~s}, 1 \mathrm{H}), 2.94(\mathrm{dd}, J=16.0,4.8 \mathrm{~Hz}, 1 \mathrm{H}), 2.48(\mathrm{dd}, J=16.0,6.8 \mathrm{~Hz}, 1 \mathrm{H})$, 2.30-2.25 (m, 1H), $0.80(\mathrm{~d}, J=6.8 \mathrm{~Hz}, 3 \mathrm{H}) ;{ }^{13} \mathrm{C} \mathrm{NMR}\left(100 \mathrm{MHz}, \mathrm{CDCl}_{3}, \mathrm{ppm}\right) \delta 144.3,143.1,129.9$, $128.3,127.33,127.26,127.0,120.2,117.2,113.8,59.5,33.5,32.0,15.3$.

Guo. Q.-S.; Du, D.-M.; X, J. Angew. Chem. Int. Ed. 2008, 47, 759.<smiles>CC1Cc2ccccc2NC1c1cccc(Cl)c1</smiles>

(+)-cis-2-(3-chlorophenyl)-3-methyl-1,2,3,4-tetrahydroquinoline (7b): $0.1024 \mathrm{~g}$ (99\% yield), Colorless oil; $[\alpha]_{\mathrm{D}}{ }^{20}=+41.8\left(c 0.38, \mathrm{CH}_{2} \mathrm{Cl}_{2}\right)\left(72 \%\right.$ ee); IR (film): $3400,1608,1586,1478 \mathrm{~cm}^{-1} ;{ }^{1} \mathrm{H}$ NMR (400 $\left.\mathrm{MHz}, \mathrm{CDCl}_{3}, \mathrm{ppm}\right) \delta 7.28(\mathrm{~s}, 1 \mathrm{H}), 7.23-7.20(\mathrm{~m}, 2 \mathrm{H}), 7.18-7.15(\mathrm{~m}, 1 \mathrm{H}), 7.03-6.97(\mathrm{~m}, 2 \mathrm{H}), 6.65$ (ddd, $J$ $=7.2,7.2,0.8 \mathrm{~Hz}, 1 \mathrm{H}), 6.54(\mathrm{~d}, J=7.6 \mathrm{~Hz}, 1 \mathrm{H}), 4.46(\mathrm{~d}, J=2.4 \mathrm{~Hz}, 1 \mathrm{H}), 4.02(\mathrm{~s}, 1 \mathrm{H}), 2.96(\mathrm{dd}, J=16.0$, $4.8 \mathrm{~Hz}, 1 \mathrm{H}), 2.48(\mathrm{dd}, J=16.0,6.4 \mathrm{~Hz}, 1 \mathrm{H}), 2.30-2.22(\mathrm{~m}, 1 \mathrm{H}), 0.80(\mathrm{~d}, J=6.8 \mathrm{~Hz}, 3 \mathrm{H}) ;{ }^{13} \mathrm{C} \mathrm{NMR}(100$ $\left.\mathrm{MHz}, \mathrm{CDCl}_{3}, \mathrm{ppm}\right) \delta 145.3,143.9,134.3,129.9,129.6,127.5,127.4,127.1,125.5,120.0,117.6,114.0$, 59.2, 33.6, 32.1, 15.1; HRMS (ESI) calcd for $\mathrm{C}_{16} \mathrm{H}_{17} \mathrm{NCl}(\mathrm{M}+\mathrm{H})$ : 258.1044, Found: 258.1043.<smiles>CC1Cc2ccccc2NC1c1cccc(Br)c1</smiles>

(+)-cis-2-(3-bromophenyl)-3-methyl-1,2,3,4-tetrahydroquinoline (7c): $0.1196 \mathrm{~g}$ (99\% yield), Colorless oil; $[\alpha]_{\mathrm{D}}{ }^{20}=+21.5\left(c 0.40, \mathrm{CH}_{2} \mathrm{Cl}_{2}\right)\left(66 \%\right.$ ee); IR (film): 3398, 1608, 1587, $1475 \mathrm{~cm}^{-1} ;{ }^{1} \mathrm{H}$ NMR (400 $\left.\mathrm{MHz}, \mathrm{CDCl}_{3}, \mathrm{ppm}\right) \delta 7.46(\mathrm{~s}, 1 \mathrm{H}), 7.40(\mathrm{ddd}, J=7.6,1.6,1.6 \mathrm{~Hz}, 1 \mathrm{H}), 7.25-7.17(\mathrm{~m}, 2 \mathrm{H}), 7.05-6.99(\mathrm{~m}$, 2H), $6.67(\mathrm{ddd}, J=7.2,7.2,0.8 \mathrm{~Hz}, 1 \mathrm{H}), 6.57(\mathrm{~d}, J=8.0 \mathrm{~Hz}, 1 \mathrm{H}), 4.49(\mathrm{~d}, J=2.8 \mathrm{~Hz}, 1 \mathrm{H}), 4.06(\mathrm{~s}, 1 \mathrm{H})$, 
$2.99(\mathrm{dd}, J=16.0,4.8 \mathrm{~Hz}, 1 \mathrm{H}), 2.49(\mathrm{dd}, J=16.0,6.4 \mathrm{~Hz}, 1 \mathrm{H}), 2.33-2.24(\mathrm{~m}, 1 \mathrm{H}), 0.81(\mathrm{~d}, J=6.8 \mathrm{~Hz}$, $3 \mathrm{H}) ;{ }^{13} \mathrm{C} \mathrm{NMR}\left(100 \mathrm{MHz}, \mathrm{CDCl}_{3}, \mathrm{ppm}\right) \delta 145.6,143.9,130.43,130.37,129.97,129.95,127.2,126.0$, 122.5, 120.1, 117.7, 114.1, 59.1, 33.6, 32.2, 15.1; HRMS (ESI) calcd for $\mathrm{C}_{16} \mathrm{H}_{17} \mathrm{NBr}(\mathrm{M}+\mathrm{H})$ : 302.0539, Found: 302.0537.<smiles>CC1Cc2ccccc2NC1c1cccc(C(F)(F)F)c1</smiles>

(+)-cis-3-methyl-2-(3-(trifluoromethyl)phenyl)-1,2,3,4-tetrahydroquinoline (7d): 0.1046 g (90\% yield), Colorless oil; $[\alpha]_{\mathrm{D}}{ }^{20}=+23.7\left(c 0.59, \mathrm{CH}_{2} \mathrm{Cl}_{2}\right)\left(65 \%\right.$ ee); IR (film): $3403,1678,1608,1587 \mathrm{~cm}^{-1}$; ${ }^{1} \mathrm{H}$ NMR (400 MHz, $\left.\mathrm{CDCl}_{3}, \mathrm{ppm}\right) \delta 7.56(\mathrm{~s}, 1 \mathrm{H}), 7.51(\mathrm{dd}, J=8.0,8.0 \mathrm{~Hz}, 2 \mathrm{H}), 7.43(\mathrm{dd}, J=7.6,7.6 \mathrm{~Hz}$, 1H), 7.05-6.99 (m, 2H), $6.67(\mathrm{ddd}, J=7.6,7.6,0.8 \mathrm{~Hz}, 1 \mathrm{H}), 6.57(\mathrm{~d}, J=8.0 \mathrm{~Hz}, 1 \mathrm{H}), 4.60(\mathrm{~d}, J=2.8 \mathrm{~Hz}$, 1H), $4.09(\mathrm{~s}, 1 \mathrm{H}), 3.03(\mathrm{dd}, J=16.0,4.8 \mathrm{~Hz}, 1 \mathrm{H}), 2.50(\mathrm{dd}, J=16.4,6.0 \mathrm{~Hz}, 1 \mathrm{H}), 2.34-2.29(\mathrm{~m}, 1 \mathrm{H}), 0.81$ $(\mathrm{d}, J=6.8 \mathrm{~Hz}, 3 \mathrm{H}) ;{ }^{13} \mathrm{C} \mathrm{NMR}\left(100 \mathrm{MHz}, \mathrm{CDCl}_{3}, \mathrm{ppm}\right) \delta 144.2,143.9,130.7\left(\mathrm{q}, J_{\mathrm{C}-\mathrm{F}}=32.0 \mathrm{~Hz}\right), 130.6$, 130.0, 128.9, 127.2, $124.4\left(\mathrm{q}, J_{\mathrm{C}-\mathrm{F}}=270.7 \mathrm{~Hz}\right), 124.2\left(\mathrm{q}, J_{\mathrm{C}-\mathrm{F}}=3.7 \mathrm{~Hz}\right), 124.1\left(\mathrm{q}, J_{\mathrm{C}-\mathrm{F}}=3.7 \mathrm{~Hz}\right), 120.0$, 117.8, 114.2, 59.3, 33.7, 32.2, 14.9; ${ }^{19} \mathrm{~F}$ NMR (471 MHz, $\left.\mathrm{CDCl}_{3}, \mathrm{ppm}\right) \delta-62.5$; HRMS (ESI) calcd for $\mathrm{C}_{17} \mathrm{H}_{17} \mathrm{NF}_{3}(\mathrm{M}+\mathrm{H}):$ 292.1308, Found: 292.1307.<smiles>Cc1cccc(C2Nc3ccccc3CC2C)c1</smiles>

(+)-cis-3-methyl-2-(m-tolyl)-1,2,3,4-tetrahydroquinoline (7e): 0.0928 g (98\% yield), Colorless oil; $[\alpha]_{\mathrm{D}}{ }^{20}=+19.0\left(c 0.52, \mathrm{CH}_{2} \mathrm{Cl}_{2}\right)\left(73 \%\right.$ ee); IR (film): 3404, 1608, 1586, $1484 \mathrm{~cm}^{-1} ;{ }^{1} \mathrm{H}$ NMR $(400 \mathrm{MHz}$, $\left.\mathrm{CDCl}_{3}, \mathrm{ppm}\right) \delta$ 7.21-7.19 (m, 1H), 7.10-7.06 (m, 3H), 7.03-6.98 (m, 2H), $6.64(\mathrm{ddd}, J=7.2,7.2,0.8 \mathrm{~Hz}$, 1H), $6.54(\mathrm{~d}, J=7.6 \mathrm{~Hz}, 1 \mathrm{H}), 4.78(\mathrm{~d}, J=3.6 \mathrm{~Hz}, 1 \mathrm{H}), 4.06(\mathrm{~s}, 1 \mathrm{H}), 2.99(\mathrm{dd}, J=16.0,4.8 \mathrm{~Hz}, 1 \mathrm{H}), 2.51$ $(\mathrm{dd}, J=16.0,6.0 \mathrm{~Hz}, 1 \mathrm{H}), 2.34(\mathrm{~s}, 3 \mathrm{H}), 2.30-2.24(\mathrm{~m}, 1 \mathrm{H}), 0.81(\mathrm{~d}, J=6.8 \mathrm{~Hz}, 3 \mathrm{H}) ;{ }^{13} \mathrm{C} \mathrm{NMR}(100 \mathrm{MHz}$, $\left.\mathrm{CDCl}_{3}, \mathrm{ppm}\right) \delta 144.4,143.1,137.9,130.0,128.3,128.0,127.0,124.4,120.2,117.3,113.9,59.5,33.9$, 32.2, 21.7, 15.0; HRMS (ESI) calcd for $\mathrm{C}_{17} \mathrm{H}_{20} \mathrm{~N}(\mathrm{M}+\mathrm{H}): 238.1590$, Found: 238.1586. 
<smiles>CC1Cc2ccccc2NC1c1ccc(Cl)cc1</smiles>

(+)-cis -2-(4-chlorophenyl)-3-methyl-1,2,3,4-tetrahydroquinoline (7f): $0.0894 \mathrm{~g}$ (87\% yield), Colorless oil; $[\alpha]_{\mathrm{D}}{ }^{20}=+21.3\left(c 0.60, \mathrm{CH}_{2} \mathrm{Cl}_{2}\right)\left(74 \%\right.$ ee); IR (film): $3405,1608,1587,1486 \mathrm{~cm}^{-1} ;{ }^{1} \mathrm{H}$ NMR (400 $\left.\mathrm{MHz}, \mathrm{CDCl}_{3}, \mathrm{ppm}\right) \delta 7.26(\mathrm{~d}, J=8.4 \mathrm{~Hz}, 2 \mathrm{H}), 7.26(\mathrm{~d}, J=8.4 \mathrm{~Hz}, 2 \mathrm{H}), 7.03-6.96(\mathrm{~m}, 2 \mathrm{H}), 6.65(\mathrm{dd}, J=$ 7.2, 7.2 Hz, 1H), $6.53(\mathrm{~d}, J=8.0 \mathrm{~Hz}, 1 \mathrm{H}), 4.44(\mathrm{~d}, J=3.2 \mathrm{~Hz}, 1 \mathrm{H}), 4.05(\mathrm{~s}, 1 \mathrm{H}), 2.92(\mathrm{dd}, J=16.0,4.8$ $\mathrm{Hz}, 1 \mathrm{H}), 2.45(\mathrm{dd}, J=16.0,6.8 \mathrm{~Hz}, 1 \mathrm{H}), 2.28-2.23(\mathrm{~m}, 1 \mathrm{H}), 0.79(\mathrm{~d}, J=6.8 \mathrm{~Hz}, 3 \mathrm{H}) ;{ }^{13} \mathrm{C} \mathrm{NMR}(100$ $\left.\mathrm{MHz}, \mathrm{CDCl}_{3}, \mathrm{ppm}\right) \delta 144.0,141.6,132.9,129.9,128.7,128.4,127.2,120.1,117.5,113.9,59.0,33.1$, 32.0, 15.4; HRMS (ESI) calcd for $\mathrm{C}_{16} \mathrm{H}_{17} \mathrm{NCl}(\mathrm{M}+\mathrm{H})$ : 258.1044, Found: 258.1042 .<smiles>CC1Cc2ccccc2NC1c1ccc(Br)cc1</smiles>

(+)-cis-2-(4-bromophenyl)-3-methyl-1,2,3,4-tetrahydroquinoline (7g): $0.1174 \mathrm{~g}$ (97\% yield), Colorless oil; $[\alpha]_{\mathrm{D}}{ }^{20}=+24.2\left(c 0.36, \mathrm{CH}_{2} \mathrm{Cl}_{2}\right)\left(77 \%\right.$ ee); IR (film): $3400,1607,1586,1486 \mathrm{~cm}^{-1} ;{ }^{1} \mathrm{H}$ NMR (400 $\left.\mathrm{MHz}, \mathrm{CDCl}_{3}, \mathrm{ppm}\right) \delta 7.42(\mathrm{~d}, J=8.4 \mathrm{~Hz}, 2 \mathrm{H}), 7.15(\mathrm{~d}, J=8.4 \mathrm{~Hz}, 2 \mathrm{H}), 7.03-6.97(\mathrm{~m}, 2 \mathrm{H}), 6.65(\mathrm{ddd}, J=$ 7.2, 7.2, $0.8 \mathrm{~Hz}, 1 \mathrm{H}), 6.53(\mathrm{~d}, J=8.0 \mathrm{~Hz}, 1 \mathrm{H}), 4.44(\mathrm{~d}, J=3.2 \mathrm{~Hz}, 1 \mathrm{H}), 4.06(\mathrm{~s}, 1 \mathrm{H}), 2.93(\mathrm{dd}, J=16.4$, $4.8 \mathrm{~Hz}, 1 \mathrm{H}), 2.45(\mathrm{dd}, J=16.0,6.8 \mathrm{~Hz}, 1 \mathrm{H}), 2.28-2.24(\mathrm{~m}, 1 \mathrm{H}), 0.79(\mathrm{~d}, J=6.8 \mathrm{~Hz}, 3 \mathrm{H}) ;{ }^{13} \mathrm{C} \mathrm{NMR}(100$ $\left.\mathrm{MHz}, \mathrm{CDCl}_{3}, \mathrm{ppm}\right) \delta 144.0,142.1,131.3,129.9,129.1,127.2,121.1,120.1,117.5,113.9,59.1,33.3$, 32.0, 15.4; HRMS (ESI) calcd for $\mathrm{C}_{16} \mathrm{H}_{17} \mathrm{NBr}(\mathrm{M}+\mathrm{H})$ : 302.0539 , Found: 302.0536.<smiles>CC1Cc2ccccc2NC1c1ccc(C(F)(F)F)cc1</smiles>

(+)-cis-3-methyl-2-(4-(trifluoromethyl)phenyl)-1,2,3,4-tetrahydroquinoline $\quad$ (7h): 0.1075 g $\quad$ (92\% yield), Colorless oil; $[\alpha]_{\mathrm{D}}{ }^{20}=+19.1\left(c 0.44, \mathrm{CH}_{2} \mathrm{Cl}_{2}\right)\left(70 \%\right.$ ee); IR (film): $3403,1608,1587,1489 \mathrm{~cm}^{-1}$; ${ }^{1} \mathrm{H}$ NMR (400 MHz, $\left.\mathrm{CDCl}_{3}, \mathrm{ppm}\right) \delta 7.57(\mathrm{~d}, J=8.0 \mathrm{~Hz}, 2 \mathrm{H}), 7.41(\mathrm{~d}, J=8.0 \mathrm{~Hz}, 2 \mathrm{H}), 7.06-6.99(\mathrm{~m}, 2 \mathrm{H})$, 
$6.68(\mathrm{ddd}, J=7.2,7.2,0.8 \mathrm{~Hz}, 1 \mathrm{H}), 6.58(\mathrm{~d}, J=8.0 \mathrm{~Hz}, 1 \mathrm{H}), 4.57(\mathrm{~d}, J=2.4 \mathrm{~Hz}, 1 \mathrm{H}), 4.12(\mathrm{~s}, 1 \mathrm{H}), 2.97$ $(\mathrm{dd}, J=16.0,4.8 \mathrm{~Hz}, 1 \mathrm{H}), 2.48(\mathrm{dd}, J=16.4,6.8 \mathrm{~Hz}, 1 \mathrm{H}), 2.38-2.29(\mathrm{~m}, 1 \mathrm{H}), 0.81(\mathrm{~d}, J=6.8 \mathrm{~Hz}, 3 \mathrm{H})$; ${ }^{13} \mathrm{C} \mathrm{NMR}\left(100 \mathrm{MHz}, \mathrm{CDCl}_{3}, \mathrm{ppm}\right) \delta 147.2,143.9,130.0,129.6\left(\mathrm{q}, J_{\mathrm{C}-\mathrm{F}}=32.2 \mathrm{~Hz}\right), 127.7,127.2,125.3(\mathrm{q}$, $\left.J_{\mathrm{C}-\mathrm{F}}=3.8 \mathrm{~Hz}\right), 124.4\left(\mathrm{q}, J_{\mathrm{C}-\mathrm{F}}=270.2 \mathrm{~Hz}\right), 120.1,117.7,114.0,59.3,33.4,32.0,15.4 ;{ }^{19} \mathrm{~F}$ NMR $(471 \mathrm{MHz}$, $\left.\mathrm{CDCl}_{3}, \mathrm{ppm}\right) \delta$-62.4; HRMS (ESI) calcd for $\mathrm{C}_{17} \mathrm{H}_{17} \mathrm{NF}_{3}(\mathrm{M}+\mathrm{H})$ : 292.1308, Found: 292.1305.

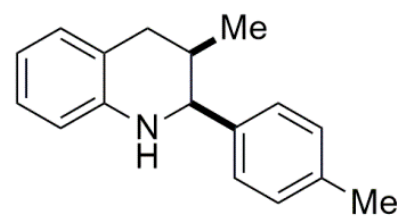

(+)-cis-3-methyl-2-(p-tolyl)-1,2,3,4-tetrahydroquinoline (7i): 0.0890 g (94\% yield), White solid, m.p. $53-56{ }^{\circ} \mathrm{C} ;[\alpha]_{\mathrm{D}}{ }^{20}=+26.7\left(c 0.51, \mathrm{CH}_{2} \mathrm{Cl}_{2}\right)\left(69 \%\right.$ ee); IR (film): $3404,1608,1586,1488 \mathrm{~cm}^{-1} ;{ }^{1} \mathrm{H}$ NMR $\left(400 \mathrm{MHz}, \mathrm{CDCl}_{3}, \mathrm{ppm}\right) \delta 7.18(\mathrm{~d}, J=8.0 \mathrm{~Hz}, 2 \mathrm{H}), 7.12(\mathrm{~d}, J=8.0 \mathrm{~Hz}, 2 \mathrm{H}), 7.03-6.98(\mathrm{~m}, 2 \mathrm{H}), 6.64$ (ddd, $J=7.2,7.2,0.8 \mathrm{~Hz}, 1 \mathrm{H}), 6.55(\mathrm{~d}, J=8.0 \mathrm{~Hz}, 1 \mathrm{H}), 4.44$ (d, $J=3.2 \mathrm{~Hz}, 1 \mathrm{H}), 4.09$ (s, $1 \mathrm{H}), 2.96$ (dd, $J$ $=16.0,4.8 \mathrm{~Hz}, 1 \mathrm{H}), 2.49(\mathrm{dd}, J=16.0,6.8 \mathrm{~Hz}, 1 \mathrm{H}), 2.34(\mathrm{~s}, 3 \mathrm{H}), 2.31-2.26(\mathrm{~m}, 1 \mathrm{H}), 0.81(\mathrm{~d}, J=6.8 \mathrm{~Hz}$, $3 \mathrm{H}) ;{ }^{13} \mathrm{C} \mathrm{NMR}\left(100 \mathrm{MHz}, \mathrm{CDCl}_{3}, \mathrm{ppm}\right) \delta 144.4,140.1,136.9,129.9,129.0,127.3,127.0,120.2,117.2$, 113.8, 59.3, 33.6, 32.1, 21.2, 15.3; HRMS (ESI) calcd for $\mathrm{C}_{17} \mathrm{H}_{20} \mathrm{~N}(\mathrm{M}+\mathrm{H})$ : 238.1590, Found: 238.1589.

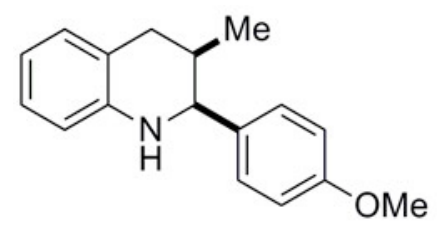

(+)-cis-2-(4-methoxyphenyl)-3-methyl-1,2,3,4-tetrahydroquinoline (7j): 0.0975 g (96\% yield), White solid, m.p. $100-102{ }^{\circ} \mathrm{C} ;[\alpha]_{\mathrm{D}}{ }^{20}=+25.7\left(c 0.65, \mathrm{CH}_{2} \mathrm{Cl}_{2}\right)\left(65 \%\right.$ ee); IR (film): $3399,1608,1585,1514 \mathrm{~cm}^{-1}$; ${ }^{1} \mathrm{H}$ NMR $\left(400 \mathrm{MHz}, \mathrm{CDCl}_{3}, \mathrm{ppm}\right) \delta 7.19(\mathrm{~d}, J=8.8 \mathrm{~Hz}, 2 \mathrm{H}), 7.02-6.97(\mathrm{~m}, 2 \mathrm{H}), 6.84(\mathrm{~d}, J=8.8 \mathrm{~Hz}, 2 \mathrm{H})$, $6.63(\mathrm{ddd}, J=7.2,7.2,0.8 \mathrm{~Hz}, 1 \mathrm{H}), 6.52(\mathrm{~d}, J=8.0 \mathrm{~Hz}, 1 \mathrm{H}), 4.44(\mathrm{~d}, J=3.6 \mathrm{~Hz}, 1 \mathrm{H}), 4.06(\mathrm{~s}, 1 \mathrm{H}), 3.78$ (s, 3H), $2.93(\mathrm{dd}, J=16.0,4.8 \mathrm{~Hz}, 1 \mathrm{H}), 2.47(\mathrm{dd}, J=16.0,6.8 \mathrm{~Hz}, 1 \mathrm{H}), 2.27-2.22(\mathrm{~m}, 1 \mathrm{H}), 0.80(\mathrm{~d}, J=$ $6.8 \mathrm{~Hz}, 3 \mathrm{H}) ;{ }^{13} \mathrm{C} \mathrm{NMR}\left(100 \mathrm{MHz}, \mathrm{CDCl}_{3}, \mathrm{ppm}\right) \delta 158.9,144.4,135.2,129.9,128.4,127.0,120.2,117.2$, 113.8, 113.6, 59.0, 55.4, 33.5, 32.1, 15.4; HRMS (ESI) calcd for $\mathrm{C}_{17} \mathrm{H}_{20} \mathrm{ON}(\mathrm{M}+\mathrm{H})$ : 254.1539, Found: 254.1539. 


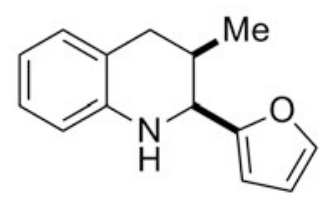

(-)-cis-2-(furan-2-yl)-3-methyl-1,2,3,4-tetrahydroquinoline (7k): 0.0799 g (94\% yield), Colorless oil; $[\alpha]_{\mathrm{D}}{ }^{20}=-22.9\left(c 0.61, \mathrm{CH}_{2} \mathrm{Cl}_{2}\right)(45 \%$ ee $)$; IR (film): 3397, 1608, 1587, $1482 \mathrm{~cm}^{-1} ;{ }^{1} \mathrm{H}$ NMR $(400 \mathrm{MHz}$, $\left.\mathrm{CDCl}_{3}, \mathrm{ppm}\right) \delta 7.33(\mathrm{~s}, 1 \mathrm{H}), 7.01-6.96(\mathrm{~m}, 2 \mathrm{H}), 6.65(\mathrm{dd}, J=7.2,7.2 \mathrm{~Hz}, 1 \mathrm{H}), 6.53(\mathrm{~d}, J=8.0 \mathrm{~Hz}, 1 \mathrm{H})$, 6.31-6.30 (m, 1H), $6.12(\mathrm{~d}, J=2.0 \mathrm{~Hz}, 1 \mathrm{H}), 4.51(\mathrm{~d}, J=3.2 \mathrm{~Hz}, 1 \mathrm{H}), 4.11(\mathrm{~s}, 1 \mathrm{H}), 2.87(\mathrm{dd}, J=16.0,4.8$ $\mathrm{Hz}, 1 \mathrm{H}), 2.53(\mathrm{dd}, J=16.0,8.0 \mathrm{~Hz}, 1 \mathrm{H}), 2.41-2.36(\mathrm{~m}, 1 \mathrm{H}), 0.92(\mathrm{~d}, J=6.8 \mathrm{~Hz}, 3 \mathrm{H}) ;{ }^{13} \mathrm{C} \mathrm{NMR}(100$ $\left.\mathrm{MHz}, \mathrm{CDCl}_{3}, \mathrm{ppm}\right) \delta 156.4,143.4,141.6,129.8,127.0,120.6,117.7,114.3,110.3,106.3,54.2,33.0$, 30.7, 16.2; HRMS (ESI) calcd for $\mathrm{C}_{14} \mathrm{H}_{16} \mathrm{NO}(\mathrm{M}+\mathrm{H})$ : 214.1226, Found: 214.1225 .

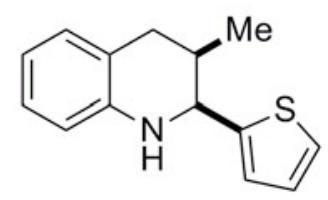

(+)-cis-3-methyl-2-(thiophen-2-yl)-1,2,3,4-tetrahydroquinoline (7l) 0.0884 g (96\% yield), White solid, m.p. $83-86{ }^{\circ} \mathrm{C} ;[\alpha]_{\mathrm{D}}{ }^{20}=+65.6\left(c 0.63, \mathrm{CH}_{2} \mathrm{Cl}_{2}\right)\left(56 \%\right.$ ee); IR (film): 3388, 1608, 1586, $1482 \mathrm{~cm}^{-1} ;{ }^{1} \mathrm{H}$ NMR (400 MHz, $\left.\mathrm{CDCl}_{3}, \mathrm{ppm}\right) \delta 7.17(\mathrm{~d}, J=5.2 \mathrm{~Hz}, 1 \mathrm{H}), 7.03-6.95(\mathrm{~m}, 3 \mathrm{H}), 6.92-6.92(\mathrm{~m}, 1 \mathrm{H}), 6.67$ (dd, $J=7.2,7.2 \mathrm{~Hz}, 1 \mathrm{H}), 6.53(\mathrm{~d}, J=8.0 \mathrm{~Hz}, 1 \mathrm{H}), 4.74(\mathrm{~d}, J=3.2 \mathrm{~Hz}, 1 \mathrm{H}), 4.21(\mathrm{~s}, 1 \mathrm{H}), 2.93(\mathrm{dd}, J=16.4$, $5.2 \mathrm{~Hz}, 1 \mathrm{H}), 2.57(\mathrm{dd}, J=16.4,7.6 \mathrm{~Hz}, 1 \mathrm{H}), 2.37-2.31(\mathrm{~m}, 1 \mathrm{H}), 0.92(\mathrm{~d}, J=6.8 \mathrm{~Hz}, 3 \mathrm{H}) ;{ }^{13} \mathrm{C} \mathrm{NMR}(100$ $\left.\mathrm{MHz}, \mathrm{CDCl}_{3}, \mathrm{ppm}\right) \delta 146.3,143.4,129.8,127.1,126.7,124.5,124.1,120.5,117.9,114.5,56.1,33.0$, 32.4, 16.2; HRMS (ESI) calcd for $\mathrm{C}_{14} \mathrm{H}_{16} \mathrm{NS}(\mathrm{M}+\mathrm{H})$ : 230.0998, Found: 230.0997.<smiles>CCC1Cc2ccccc2NC1c1ccccc1</smiles>

(+)-cis-3-ethyl-2-phenyl-1,2,3,4-tetrahydroquinoline (7m): $0.0860 \mathrm{~g}$ (91\% yield), Colorless oil; $[\alpha]_{\mathrm{D}}{ }^{20}$ $=+53.8\left(c\right.$ 0.60, $\left.\mathrm{CH}_{2} \mathrm{Cl}_{2}\right)$ (80\% ee); IR (film): 3401, 1607, 1586, $1491 \mathrm{~cm}^{-1} ;{ }^{1} \mathrm{H} \mathrm{NMR}\left(400 \mathrm{MHz}, \mathrm{CDCl}_{3}\right.$, ppm) $\delta$ 7.29-7.20 (m, 5H), 7.02-6.98 (m, 2H), $6.64(\mathrm{ddd}, J=7.2,7.2,0.8 \mathrm{~Hz}, 1 \mathrm{H}), 6.51(\mathrm{~d}, J=8.0 \mathrm{~Hz}$, $1 \mathrm{H}), 4.52(\mathrm{~d}, J=3.2 \mathrm{~Hz}, 1 \mathrm{H}), 4.17(\mathrm{~s}, 1 \mathrm{H}), 2.85(\mathrm{dd}, J=16.4,4.8 \mathrm{~Hz}, 1 \mathrm{H}), 2.53(\mathrm{dd}, J=16.4,8.8 \mathrm{~Hz}$, 
$1 \mathrm{H}), 2.08-2.00(\mathrm{~m}, 1 \mathrm{H}), 1.34-1.24(\mathrm{~m}, 1 \mathrm{H}), 1.08-0.98(\mathrm{~m}, 1 \mathrm{H}), 0.88(\mathrm{~d}, J=7.2 \mathrm{~Hz}, 3 \mathrm{H}) ;{ }^{13} \mathrm{C}$ NMR $(100$ $\left.\mathrm{MHz}, \mathrm{CDCl}_{3}, \mathrm{ppm}\right) \delta 144.5,143.3,129.8,128.3,127.4,127.24,127.16,120.3,117.1,113.7,59.0,39.0$, 29.7, 22.7, 12.2; HRMS (ESI) calcd for $\mathrm{C}_{17} \mathrm{H}_{20} \mathrm{~N}(\mathrm{M}+\mathrm{H})$ : 238.1590, Found: 238.1589.

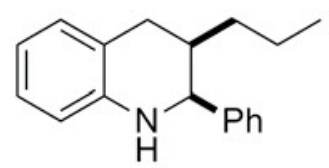

(+)-cis-2-phenyl-3-propyl-1,2,3,4-tetrahydroquinoline (7n): 0.0955 g (95\% yield), Colorless oil; $[\alpha]_{\mathrm{D}}{ }^{20}$ $=+61.9\left(c\right.$ 0.41, $\left.\mathrm{CH}_{2} \mathrm{Cl}_{2}\right)\left(80 \%\right.$ ee); IR (film): 3401, 1607, 1586, $1491 \mathrm{~cm}^{-1} ;{ }^{1} \mathrm{H} \mathrm{NMR}$ (400 MHz, $\mathrm{CDCl}_{3}$, ppm) $\delta$ 7.29-7.20 (m, 5H), 7.02-6.97 (m, 2H), $6.63(\mathrm{ddd}, J=7.2,7.2,0.8 \mathrm{~Hz}, 1 \mathrm{H}), 6.51(\mathrm{~d}, J=8.0 \mathrm{~Hz}$, $1 \mathrm{H}), 4.49(\mathrm{~d}, J=3.6 \mathrm{~Hz}, 1 \mathrm{H}), 4.17(\mathrm{~s}, 1 \mathrm{H}), 2.84(\mathrm{dd}, J=16.4,4.8 \mathrm{~Hz}, 1 \mathrm{H}), 2.53(\mathrm{dd}, J=16.4,8.8 \mathrm{~Hz}$, $1 \mathrm{H}), 2.19-2.11(\mathrm{~m}, 1 \mathrm{H}), 1.43-1.34(\mathrm{~m}, 1 \mathrm{H}), 1.28-1.16(\mathrm{~m}, 2 \mathrm{H}), 1.06-0.96(\mathrm{~m}, 1 \mathrm{H}), 0.81(\mathrm{~d}, J=7.2 \mathrm{~Hz}$, $3 \mathrm{H}) ;{ }^{13} \mathrm{C} \mathrm{NMR}\left(100 \mathrm{MHz}, \mathrm{CDCl}_{3}, \mathrm{ppm}\right) \delta 144.5,143.3,129.8,128.3,127.4,127.22,127.16,120.4,117.1$, 113.7, 59.1, 36.8, 32.1, 30.2, 20.7, 14.3; HRMS (ESI) calcd for $\mathrm{C}_{18} \mathrm{H}_{22} \mathrm{~N}(\mathrm{M}+\mathrm{H})$ : 252.1747, Found: 252.1744.

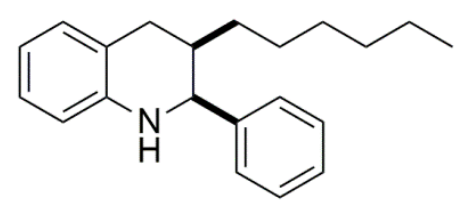

(+)-cis-3-hexyl-2-phenyl-1,2,3,4-tetrahydroquinoline (7o): 0.0865 g (74\% yield), White solid, m.p. $55-58{ }^{\circ} \mathrm{C} ;[\alpha]_{\mathrm{D}}{ }^{20}=+21.3\left(c 0.30, \mathrm{CH}_{2} \mathrm{Cl}_{2}\right)\left(80 \%\right.$ ee); IR (film): $3402,1608,1587,1492 \mathrm{~cm}^{-1} ;{ }^{1} \mathrm{H}$ NMR (400 MHz, $\left.\mathrm{CDCl}_{3}, \mathrm{ppm}\right) \delta$ 7.30-7.20 (m, 5H), 7.03-7.98 (m, 2H), $6.64(\mathrm{ddd}, J=7.2,7.2,0.8 \mathrm{~Hz}, 1 \mathrm{H})$, $6.52(\mathrm{~d}, J=8.0 \mathrm{~Hz}, 1 \mathrm{H}), 4.51(\mathrm{~d}, J=3.6 \mathrm{~Hz}, 1 \mathrm{H}), 4.19(\mathrm{~s}, 1 \mathrm{H}), 2.84(\mathrm{dd}, J=16.4,4.8 \mathrm{~Hz}, 1 \mathrm{H}), 2.53(\mathrm{dd}, J$ $=16.4,8.8 \mathrm{~Hz}, 1 \mathrm{H}), 2.18-2.10(\mathrm{~m}, 1 \mathrm{H}), 1.35-1.31(\mathrm{~m}, 1 \mathrm{H}), 1.26-1.19(\mathrm{~m}, 8 \mathrm{H}), 1.05-0.96(\mathrm{~m}, 1 \mathrm{H}), 0.84(\mathrm{t}$, $J=7.2 \mathrm{~Hz}, 3 \mathrm{H}) ;{ }^{13} \mathrm{C} \mathrm{NMR}\left(100 \mathrm{MHz}, \mathrm{CDCl}_{3}, \mathrm{ppm}\right) \delta 144.6,143.3,129.8,128.3,127.4,127.24,127.17$, 120.5, 117.1, 113.7, 59.1, 37.1, 32.0, 30.3, 29.9, 29.6, 27.6, 22.8, 14.4; HRMS (ESI) calcd for $\mathrm{C}_{21} \mathrm{H}_{28} \mathrm{~N}$ (M+H): 294.2216, Found: 294.2213. 


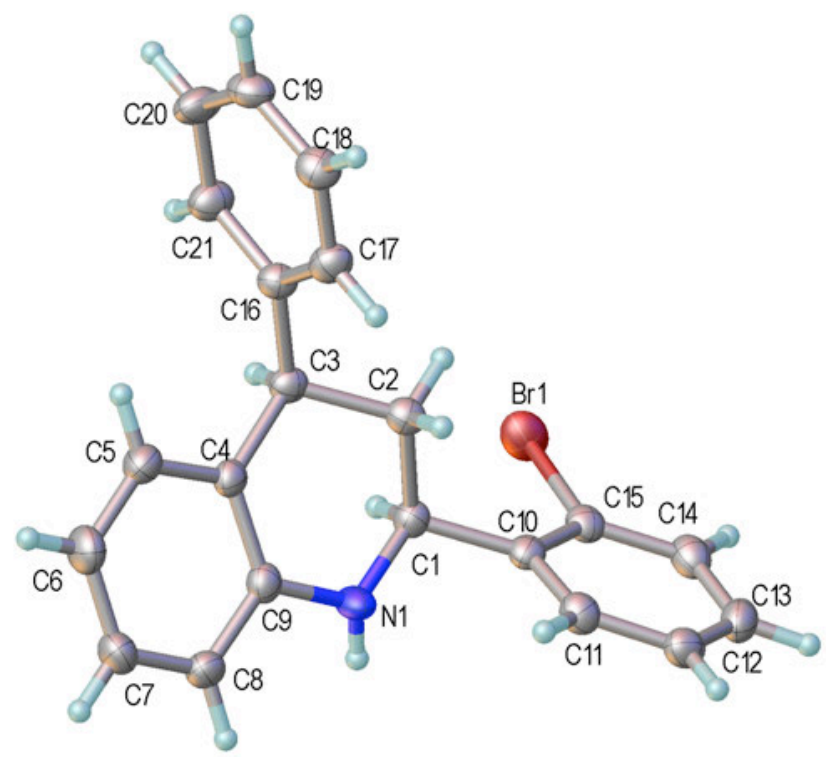

X-ray structure of compound $\mathbf{5 c}$

Table 1. Crystal data and structure refinement for $\mathbf{5 c}$

Identification code

sa4214

Empirical formula

C21 H18 Br N

Formula weight

364.27

Temperature

$173.1500 \mathrm{~K}$

Wavelength

$0.71073 \AA$

Crystal system

Monoclinic

Space group

P 1211

Unit cell dimensions

$\mathrm{a}=10.094(3) \AA$

$\mathrm{a}=90^{\circ}$.

$\mathrm{b}=14.285(4) \AA$

$\mathrm{b}=90.952(4)^{\circ}$.

$\mathrm{c}=11.689(3) \AA$

$\mathrm{g}=90^{\circ}$.

Volume

$1685.3(8) \AA^{3}$

$\mathrm{Z}$

4

Density (calculated)

$1.436 \mathrm{Mg} / \mathrm{m}^{3}$

Absorption coefficient

$2.438 \mathrm{~mm}^{-1}$

$\mathrm{F}(000)$

744

Crystal size

$0.27 \times 0.22 \times 0.15 \mathrm{~mm}^{3}$

Theta range for data collection

2.018 to $27.456^{\circ}$.

Index ranges

$-13<=\mathrm{h}<=13,-18<=\mathrm{k}<=18,-15<=\mathrm{l}<=15$

Reflections collected

19724

Independent reflections

$7604[\mathrm{R}(\mathrm{int})=0.0365]$ 
Completeness to theta $=26.000^{\circ}$

Absorption correction

Max. and min. transmission

Refinement method

Data / restraints / parameters

Goodness-of-fit on $\mathrm{F}^{2}$

Final $\mathrm{R}$ indices [I $>2 \operatorname{sigma}(\mathrm{I})]$

$\mathrm{R}$ indices (all data)

Absolute structure parameter

Extinction coefficient

Largest diff. peak and hole
$99.8 \%$

Semi-empirical from equivalents

1.0000 and 0.75096

Full-matrix least-squares on $\mathrm{F}^{2}$

7604 / 1 / 423

1.083

$\mathrm{R} 1=0.0362, \mathrm{wR} 2=0.0687$

$\mathrm{R} 1=0.0402, \mathrm{wR} 2=0.0708$

$-0.007(4)$

$\mathrm{n} / \mathrm{a}$

0.394 and -0.488 e. $\AA^{-3}$ 
Table 2, entry 1<smiles>c1ccc(C2CC(c3ccccc3)c3ccccc3N2)cc1</smiles>

HPLC Conditions: Column: Chiralcel OD-H, Daicel Chemical Industries, Ltd., Eluent: Hexanes/IPA(90/10 ); Flow rate: $0.8 \mathrm{~mL} / \mathrm{min}$; Detection: UV $254 \mathrm{~nm}$
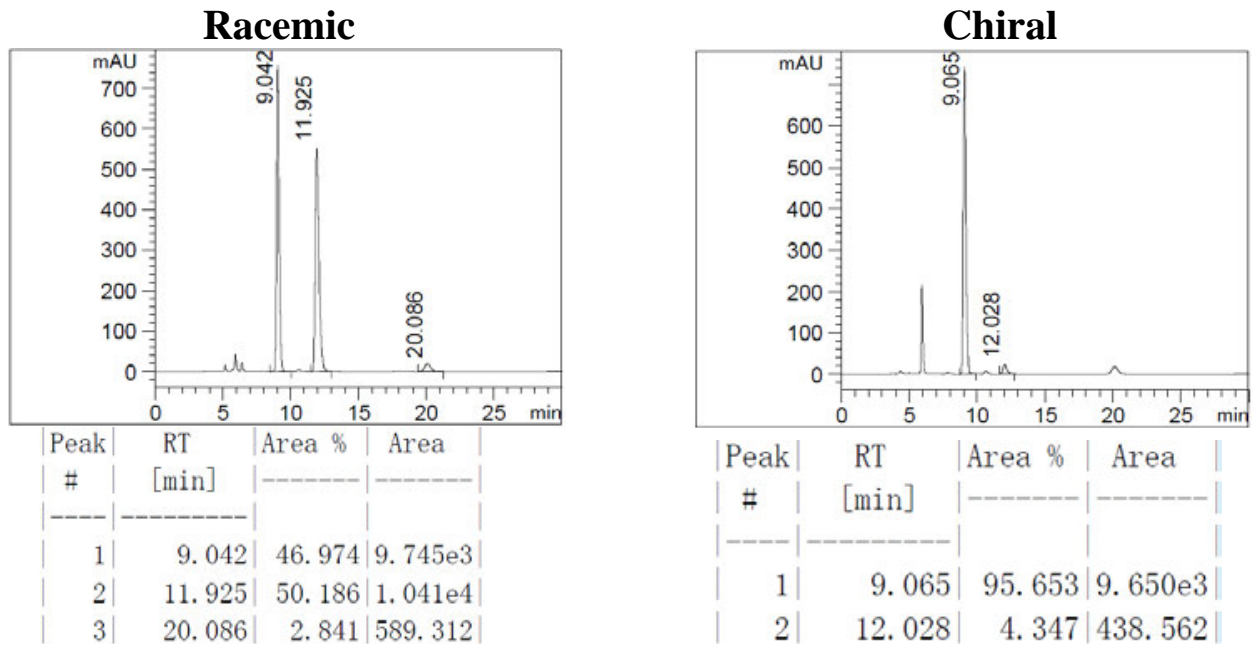

Table 2, entry 2<smiles>Clc1ccccc1C1CC(c2ccccc2)c2ccccc2N1</smiles>

HPLC Conditions: Column: Chiralcel OD-H, Daicel Chemical Industries, Ltd., Eluent: Hexanes/IPA(90/10 ); Flow rate: $0.8 \mathrm{~mL} / \mathrm{min}$; Detection: UV $254 \mathrm{~nm}$

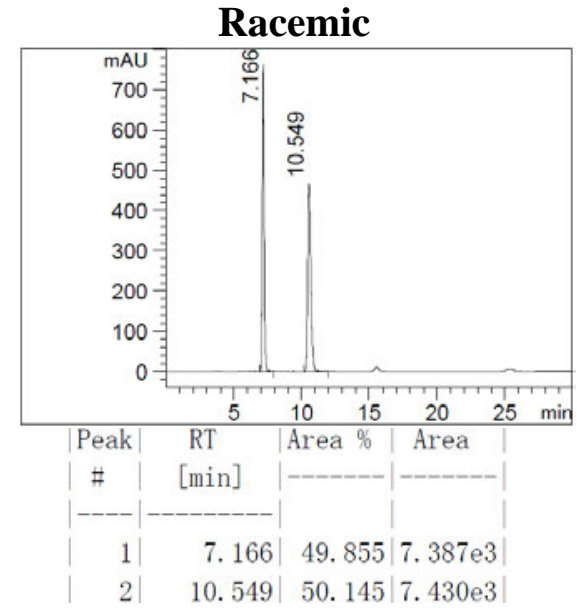

Chiral

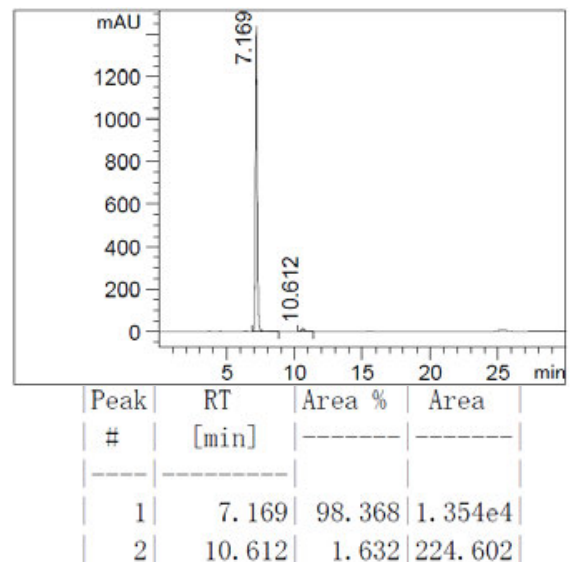


Table 2, entry 3<smiles>Brc1ccccc1C1CC(c2ccccc2)c2ccccc2N1</smiles>

HPLC Conditions: Column: Chiralcel OD-H, Daicel Chemical Industries, Ltd., Eluent: Hexanes/IPA(90/10 ); Flow rate: $0.8 \mathrm{~mL} / \mathrm{min}$; Detection: UV $254 \mathrm{~nm}$

Racemic

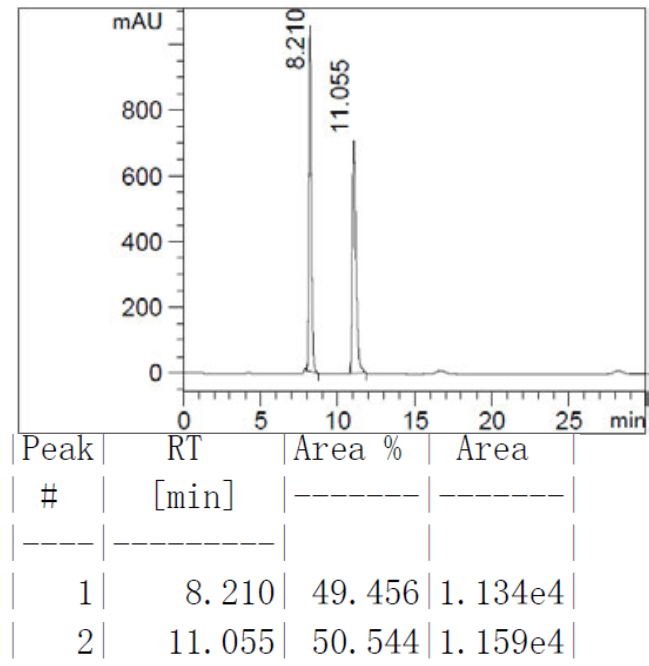

\section{Chiral}

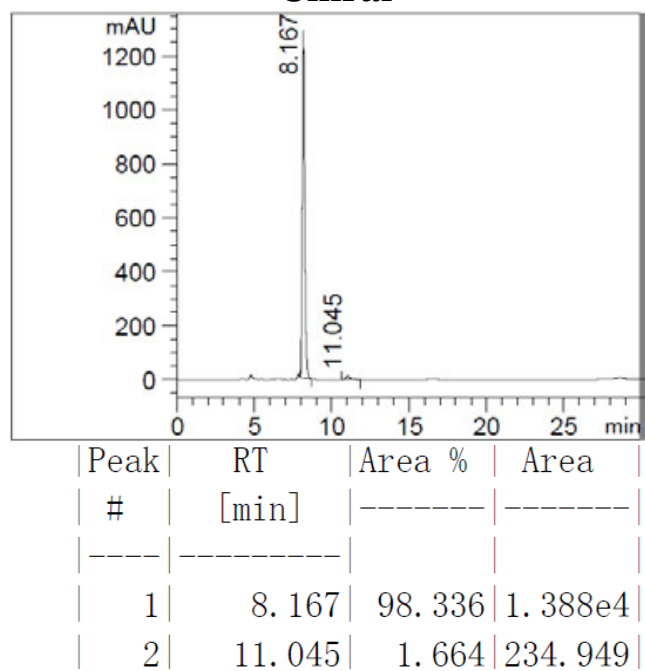

Table 2, entry 4<smiles>Clc1cccc(C2CC(c3ccccc3)c3ccccc3N2)c1</smiles>

HPLC Conditions: Column: Chiralcel OD-H, Daicel Chemical Industries, Ltd., Eluent: Hexanes/IPA(90/10 ); Flow rate: $0.8 \mathrm{~mL} / \mathrm{min}$; Detection: UV $254 \mathrm{~nm}$

Racemic

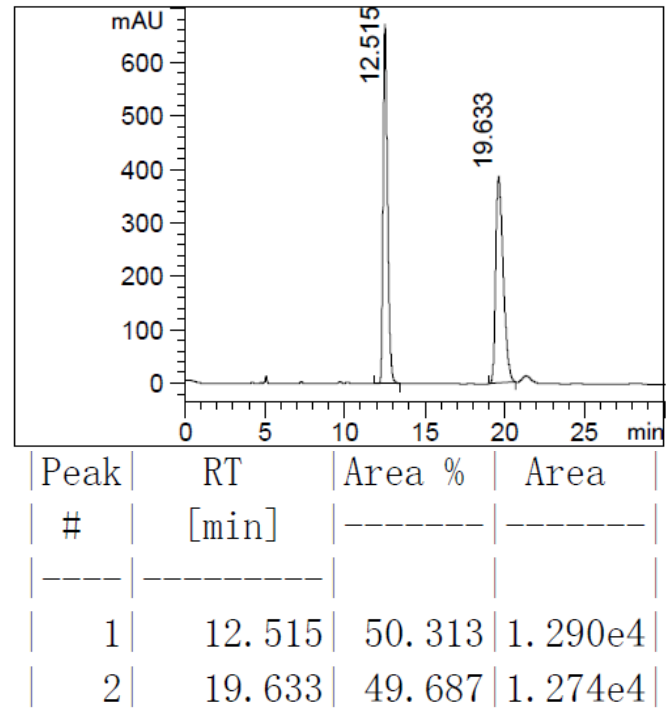

Chiral

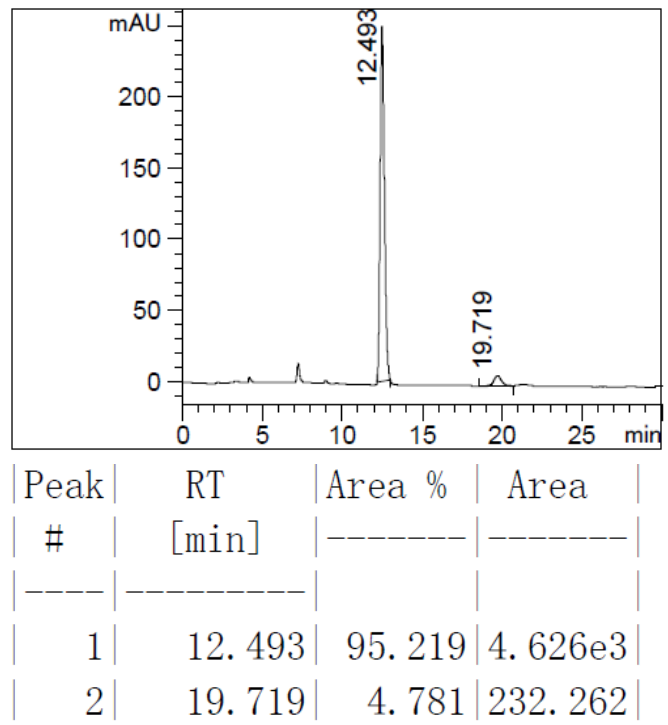


Table 2, entry 5<smiles>Brc1cccc(C2CC(c3ccccc3)c3ccccc3N2)c1</smiles>

HPLC Conditions: Column: Chiralcel OD-H, Daicel Chemical Industries, Ltd., Eluent: Hexanes/IPA(90/10 ); Flow rate: $0.8 \mathrm{~mL} / \mathrm{min}$; Detection: UV $254 \mathrm{~nm}$
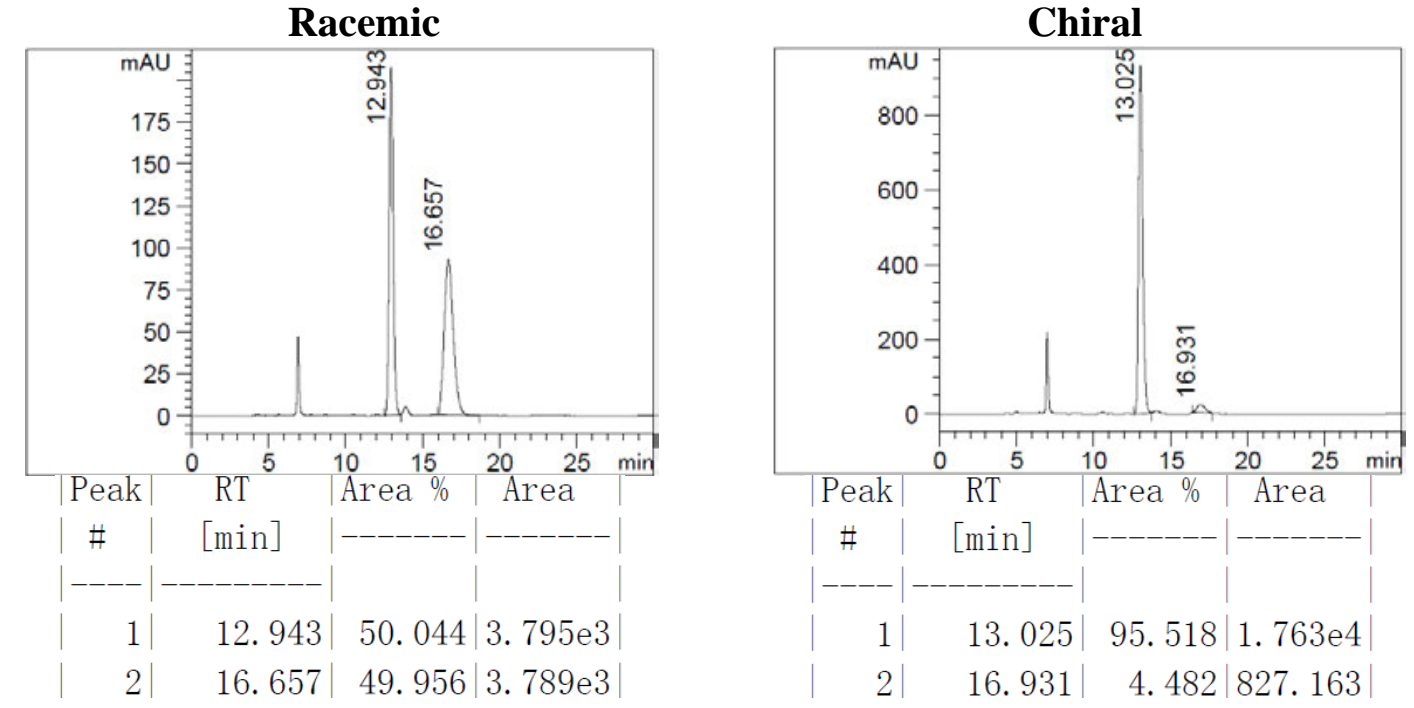

Table 2, entry 6<smiles>Cc1cccc(C2CC(c3ccccc3)c3ccccc3N2)c1</smiles>

HPLC Conditions: Column: Chiralcel OD-H, Daicel Chemical Industries, Ltd., Eluent: Hexanes/IPA(90/10 ); Flow rate: $0.8 \mathrm{~mL} / \mathrm{min}$; Detection: UV $254 \mathrm{~nm}$

Racemic

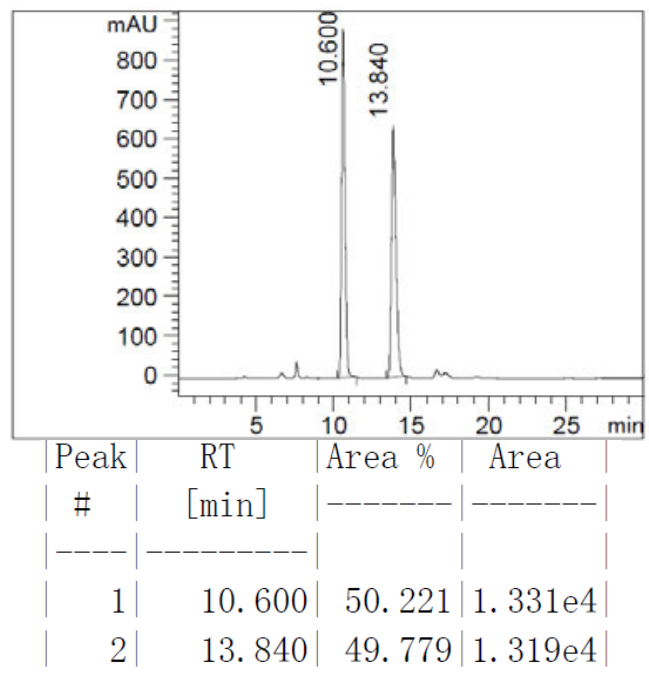

Chiral

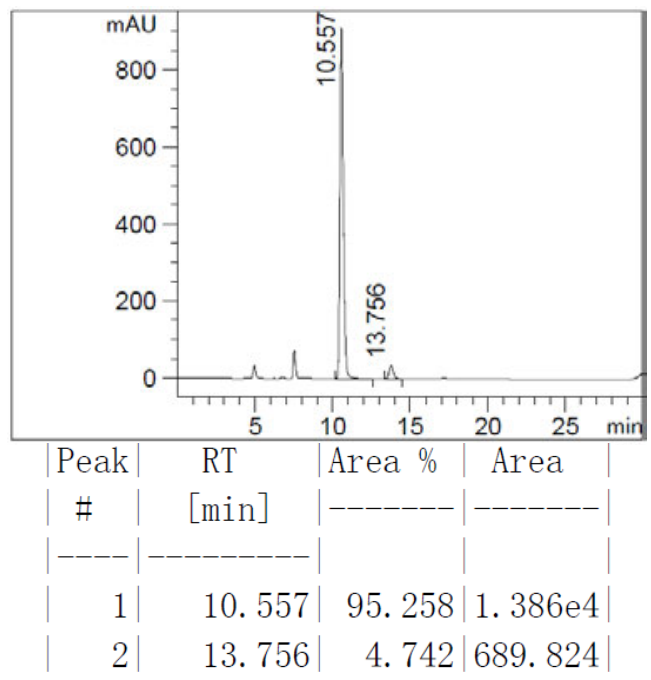


Table 2, entry 7<smiles>COc1cccc(C2CC(c3ccccc3)c3ccccc3N2)c1</smiles>

HPLC Conditions: Column: Chiralcel OD-H, Daicel Chemical Industries, Ltd., Eluent: Hexanes/IPA(90/10 ); Flow rate: $0.8 \mathrm{~mL} / \mathrm{min}$; Detection: UV $254 \mathrm{~nm}$

Racemic

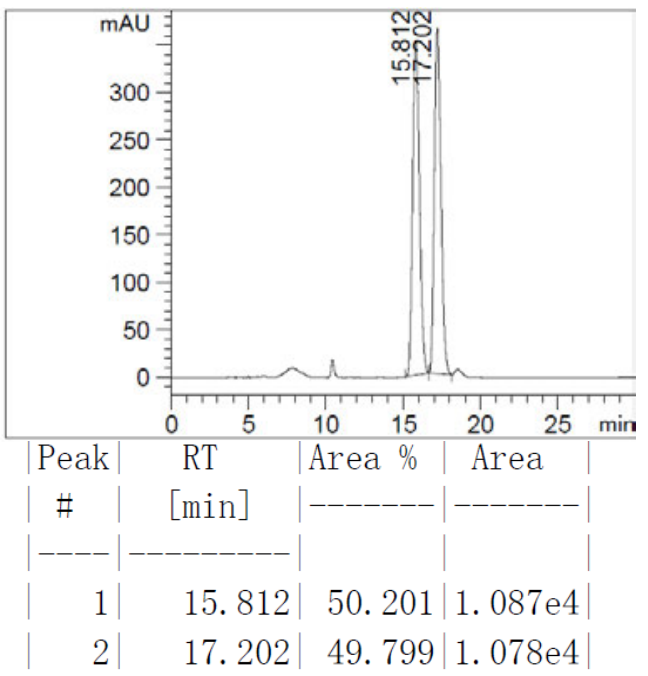

\section{Chiral}

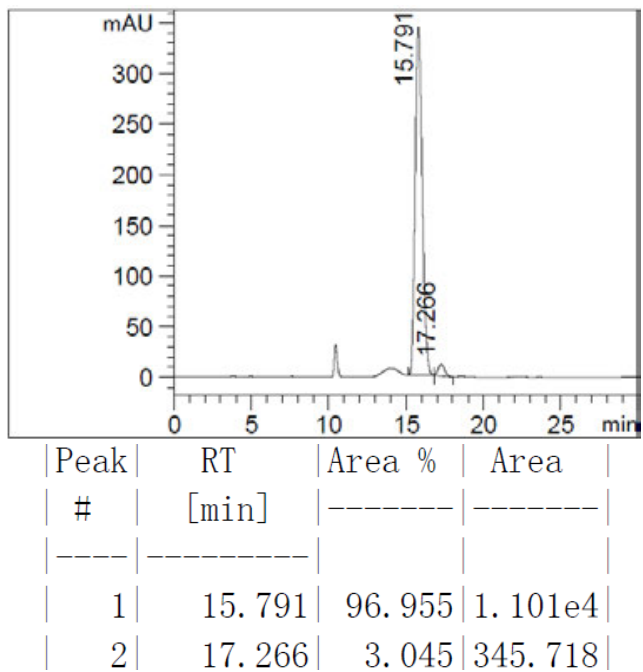

Table 2, entry 8<smiles>Clc1ccc(C2CC(c3ccccc3)c3ccccc3N2)cc1</smiles>

HPLC Conditions: Column: Chiralcel OD-H, Daicel Chemical Industries, Ltd., Eluent: Hexanes/IPA(90/10 ); Flow rate: $0.8 \mathrm{~mL} / \mathrm{min}$; Detection: UV $254 \mathrm{~nm}$

Racemic

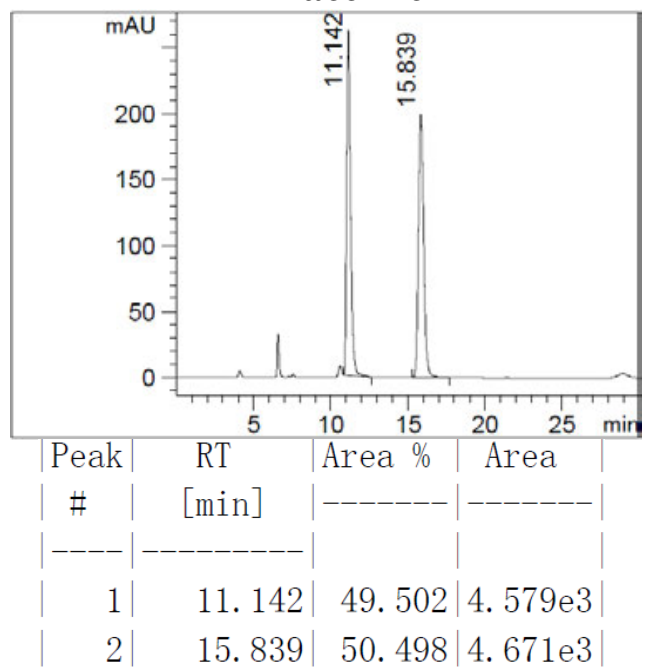

Chiral

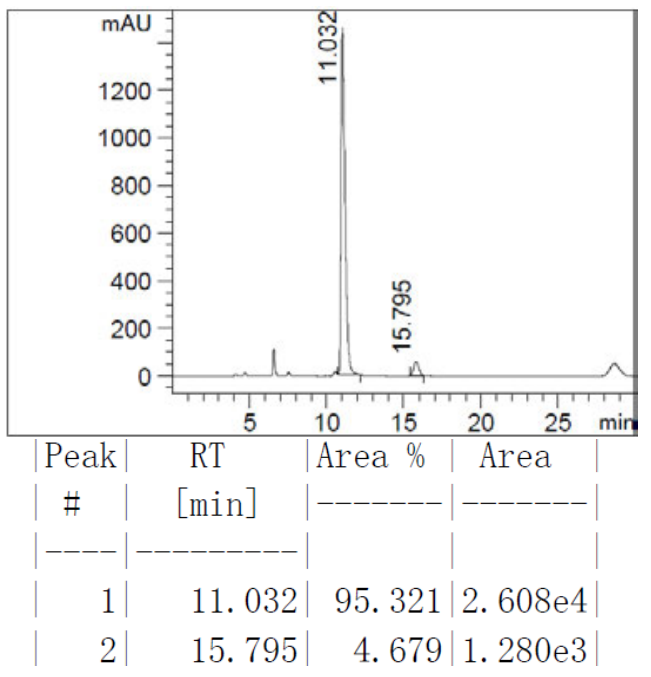


Table 2, entry 9<smiles>Brc1ccc(C2CC(c3ccccc3)c3ccccc3N2)cc1</smiles>

HPLC Conditions: Column: Chiralcel OD-H, Daicel Chemical Industries, Ltd., Eluent: Hexanes/IPA(90/10 ); Flow rate: $0.8 \mathrm{~mL} / \mathrm{min}$; Detection: UV $254 \mathrm{~nm}$

Racemic

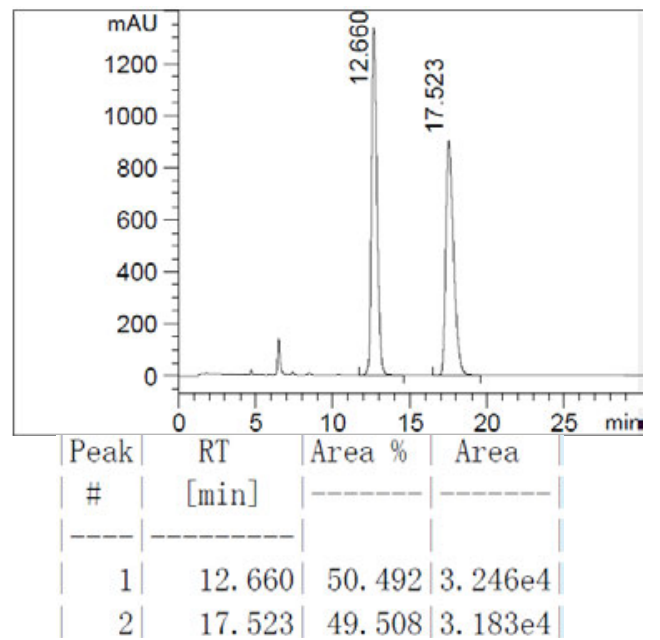

Chiral

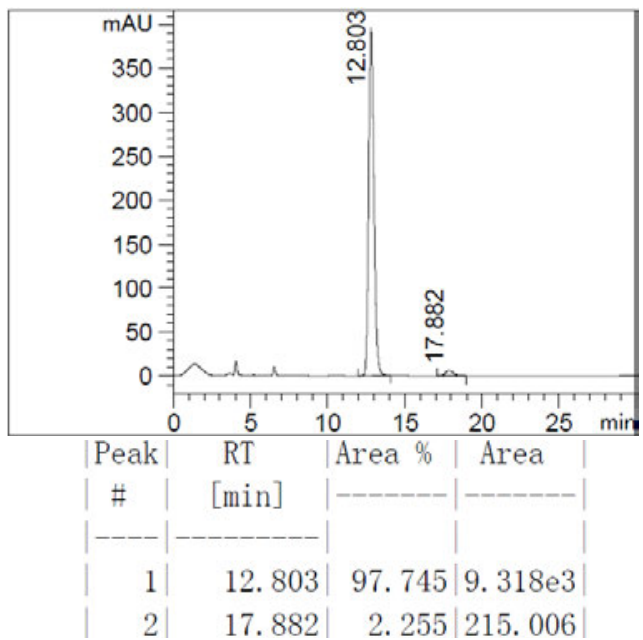

Table 2, entry 10<smiles>FC(F)(F)c1ccc(C2CC(c3ccccc3)c3ccccc3N2)cc1</smiles>

HPLC Conditions: Column: Chiralcel OD-H, Daicel Chemical Industries, Ltd., Eluent: Hexanes/IPA(90/10 ); Flow rate: $0.8 \mathrm{~mL} / \mathrm{min}$; Detection: UV $254 \mathrm{~nm}$

Racemic

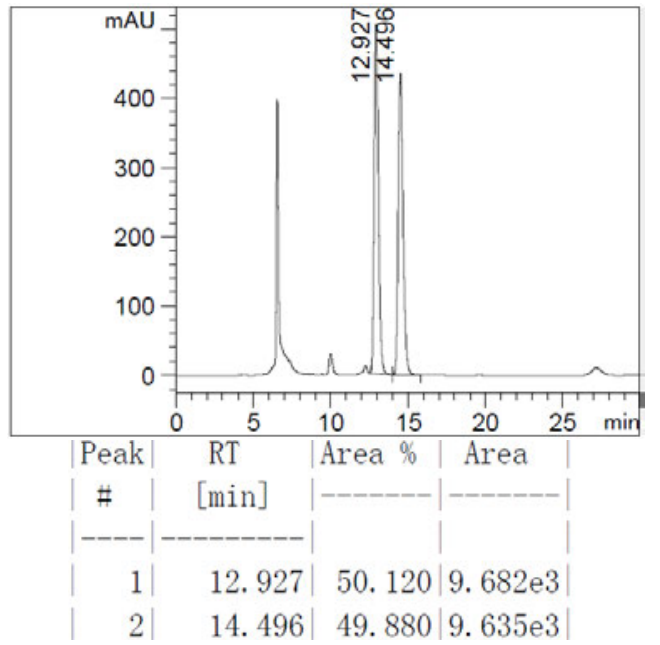

Chiral

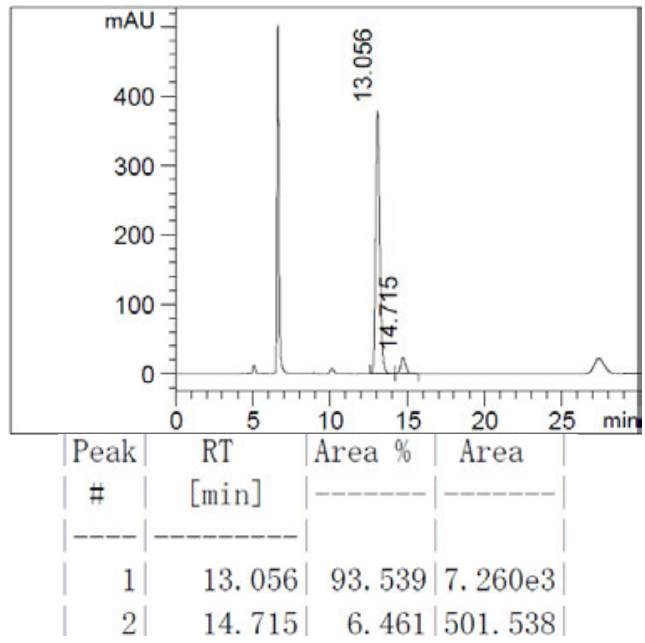


Table 2, entry 11<smiles>Cc1ccc(C2CC(c3ccccc3)c3ccccc3N2)cc1</smiles>

HPLC Conditions: Column: Chiralcel OJ-H, Daicel Chemical Industries, Ltd., Eluent: Hexanes/IPA(90/10 ); Flow rate: $0.8 \mathrm{~mL} / \mathrm{min}$; Detection: UV $254 \mathrm{~nm}$

Racemic

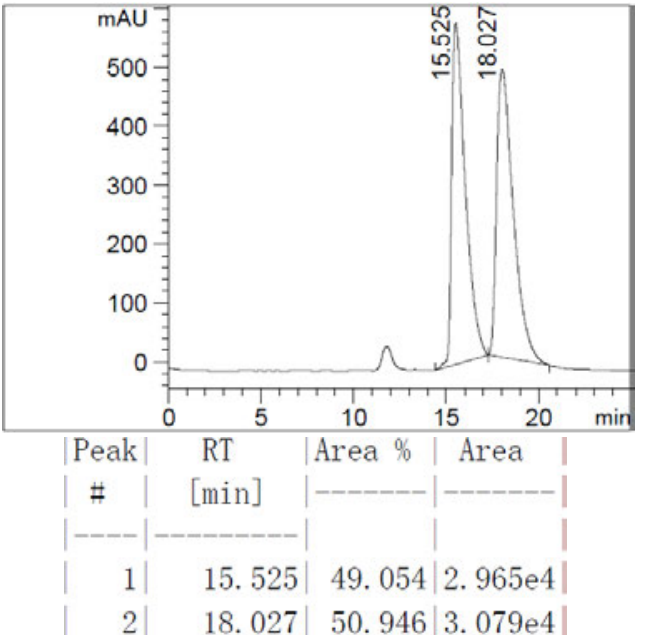

Chiral

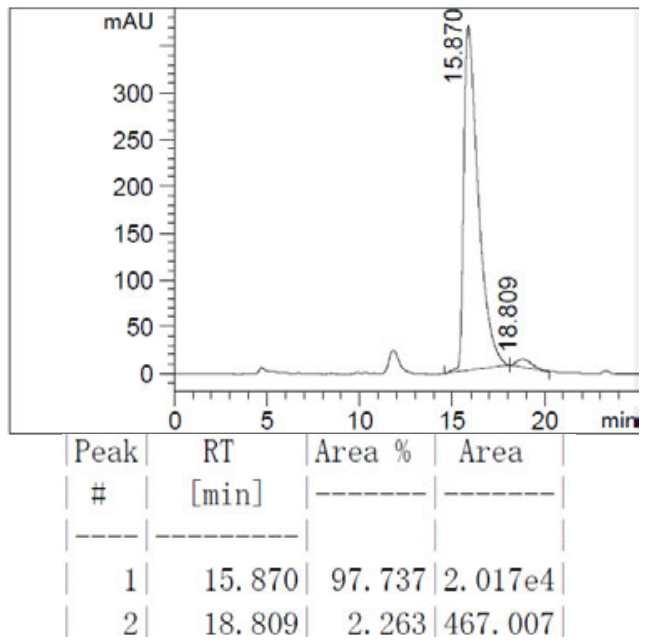

Table 2, entry 12<smiles>c1ccc(-c2ccc(C3CC(c4ccccc4)c4ccccc4N3)cc2)cc1</smiles>

HPLC Conditions: Column: Chiralcel OD-H, Daicel Chemical Industries, Ltd., Eluent: Hexanes/IPA(90/10 ); Flow rate: $0.8 \mathrm{~mL} / \mathrm{min}$; Detection: UV $254 \mathrm{~nm}$

Racemic

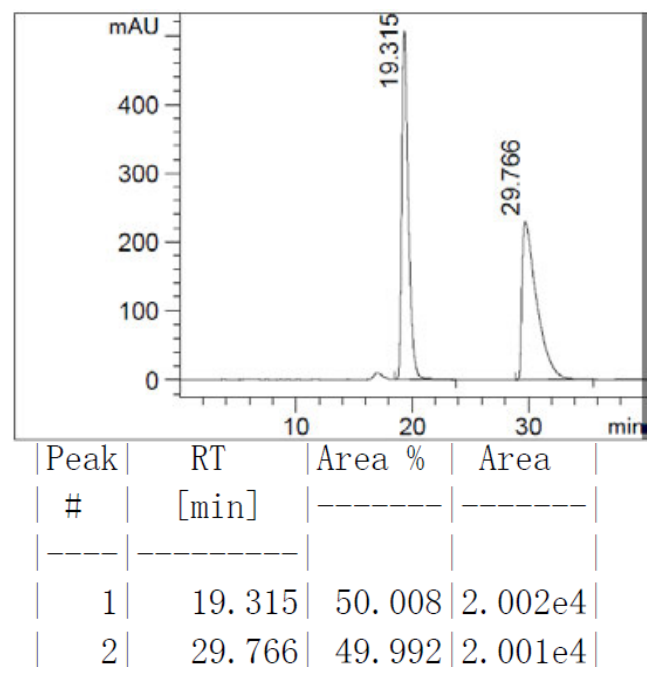

Chiral

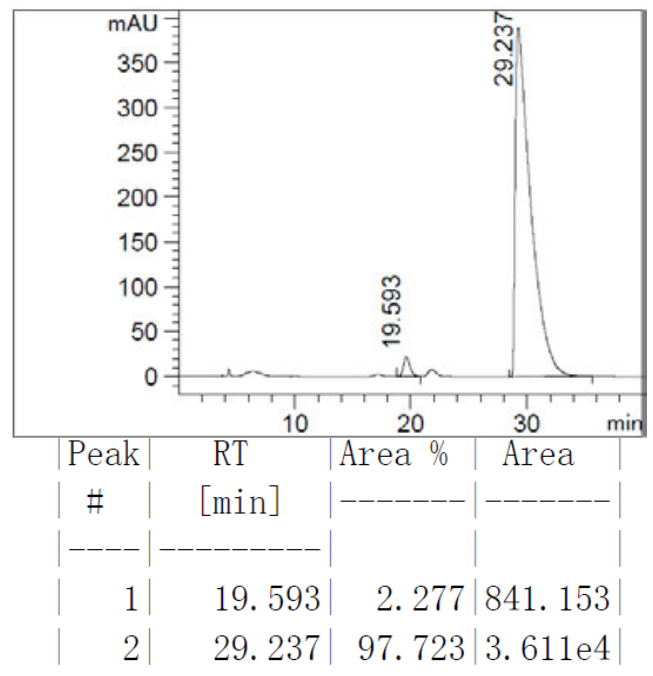


Table 2, entry 13<smiles>Cc1ccc(C2CC(c3ccccc3)c3ccccc3N2)cc1C</smiles>

HPLC Conditions: Column: Chiralcel OD-H, Daicel Chemical Industries, Ltd., Eluent: Hexanes/IPA(90/10 ); Flow rate: $0.8 \mathrm{~mL} / \mathrm{min}$; Detection: UV $254 \mathrm{~nm}$
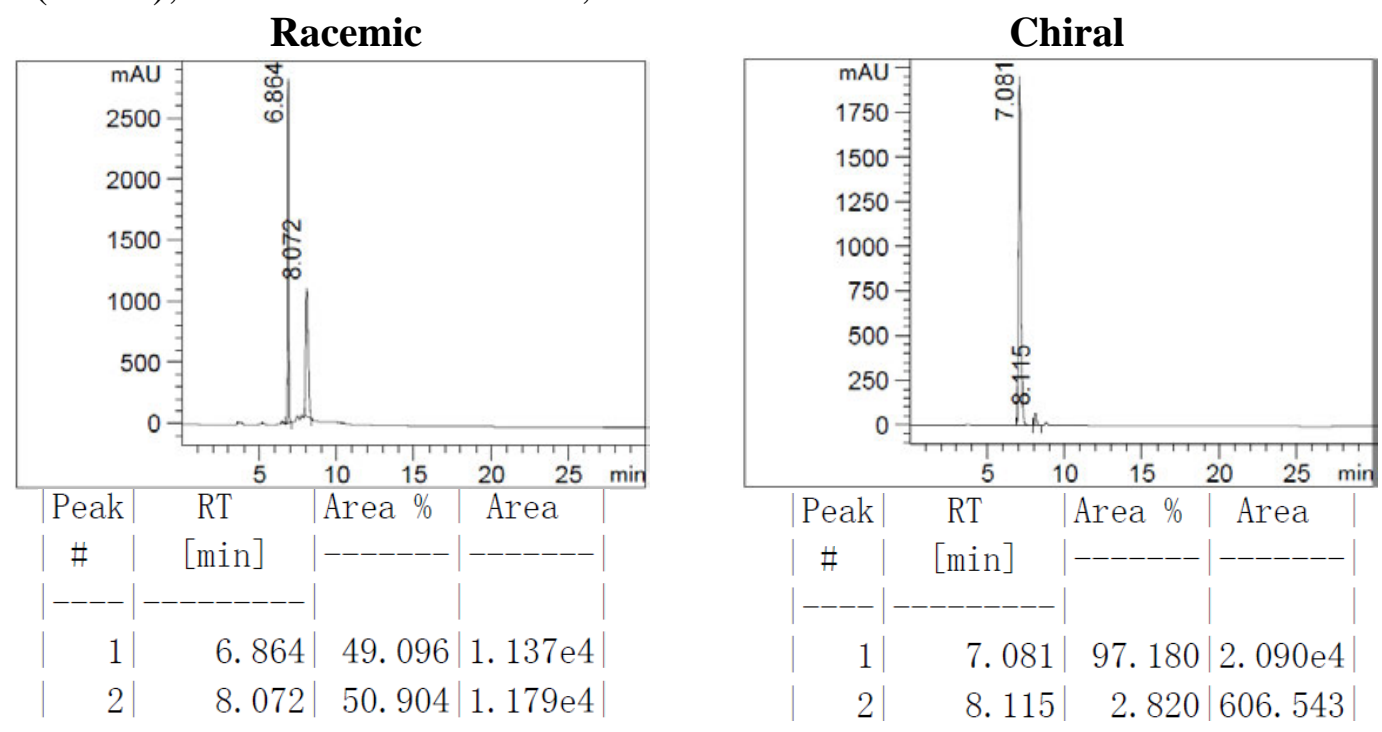

Table 2, entry 14<smiles>c1ccc(C2CC(c3ccc4ccccc4c3)Nc3ccccc32)cc1</smiles>

HPLC Conditions: Column: Chiralcel OD-H, Daicel Chemical Industries, Ltd., Eluent: Hexanes/IPA(90/10 ); Flow rate: $0.8 \mathrm{~mL} / \mathrm{min}$; Detection: UV $254 \mathrm{~nm}$

Racemic

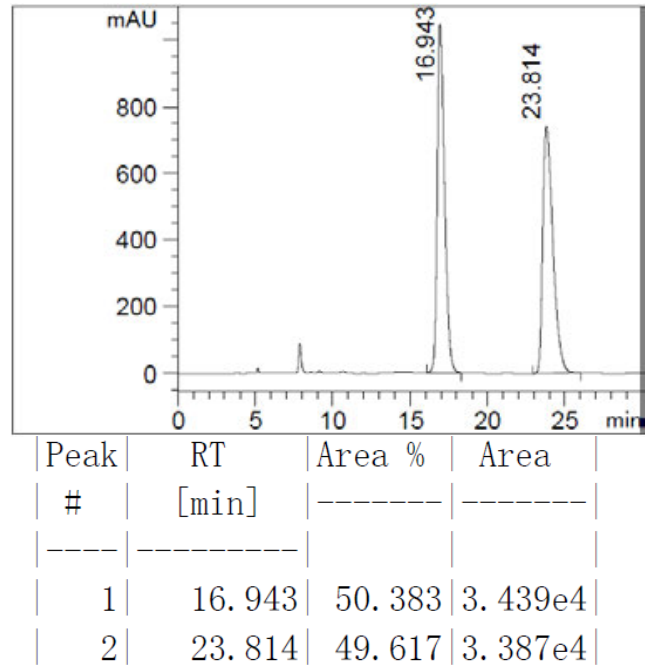

Chiral

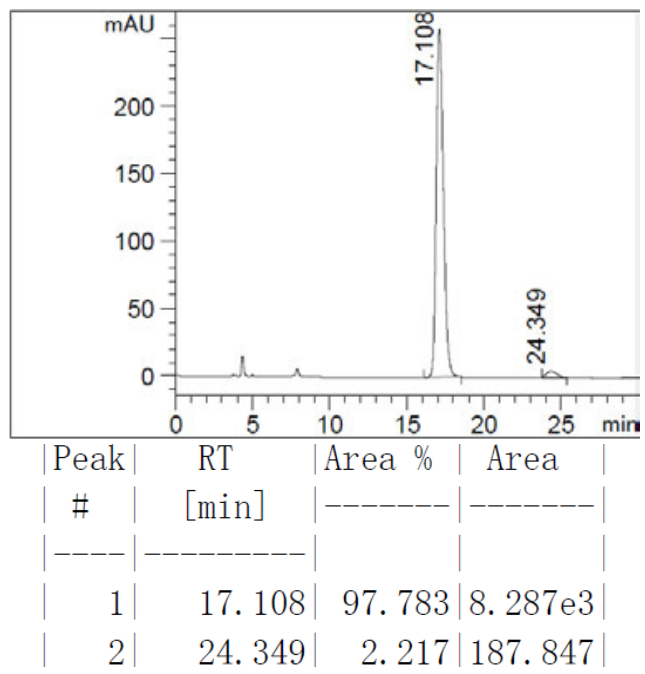


Table 2, entry 15<smiles>c1ccc(C2CC(c3ccco3)Nc3ccccc32)cc1</smiles>

HPLC Conditions: Column: Chiralcel OD-H, Daicel Chemical Industries, Ltd., Eluent: Hexanes/IPA(90/10 ); Flow rate: $0.8 \mathrm{~mL} / \mathrm{min}$; Detection: UV $254 \mathrm{~nm}$

Racemic

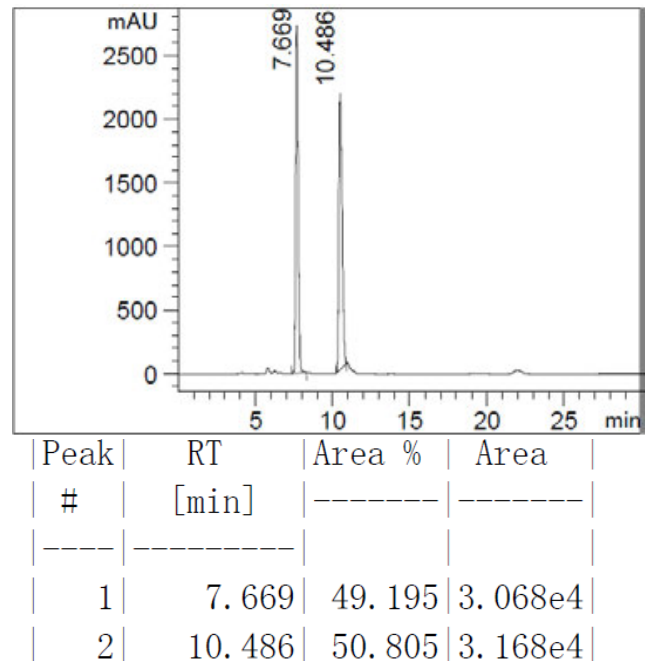

Chiral

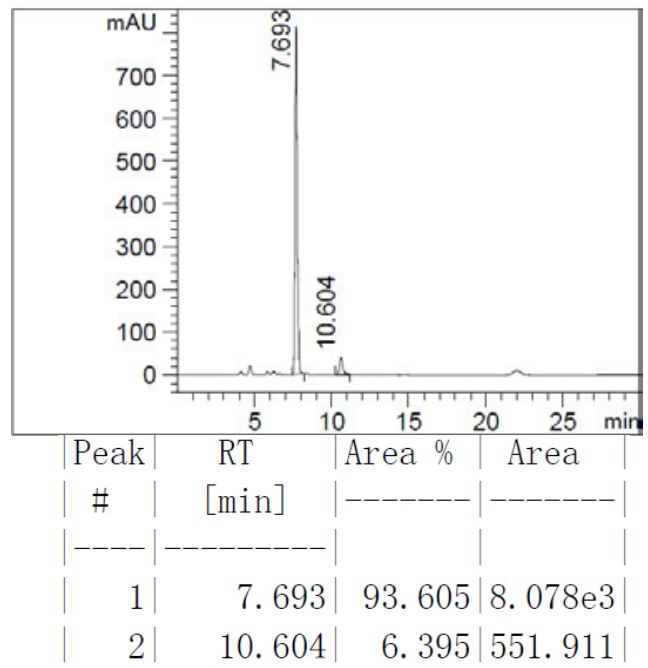

Table 2, entry 16<smiles>c1ccc(C2CC(c3cccs3)Nc3ccccc32)cc1</smiles>

HPLC Conditions: Column: Chiralcel OD-H, Daicel Chemical Industries, Ltd., Eluent: Hexanes/IPA(90/10 ); Flow rate: $0.8 \mathrm{~mL} / \mathrm{min}$; Detection: UV $254 \mathrm{~nm}$

\section{Racemic}

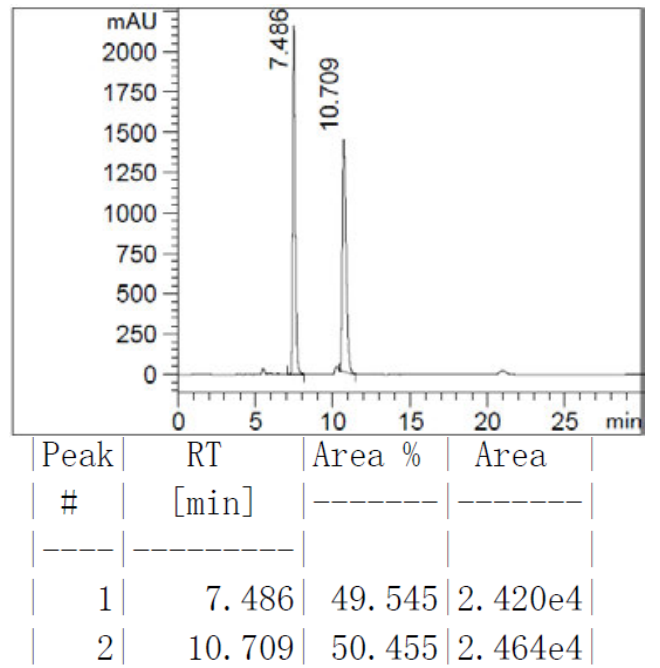

Chiral

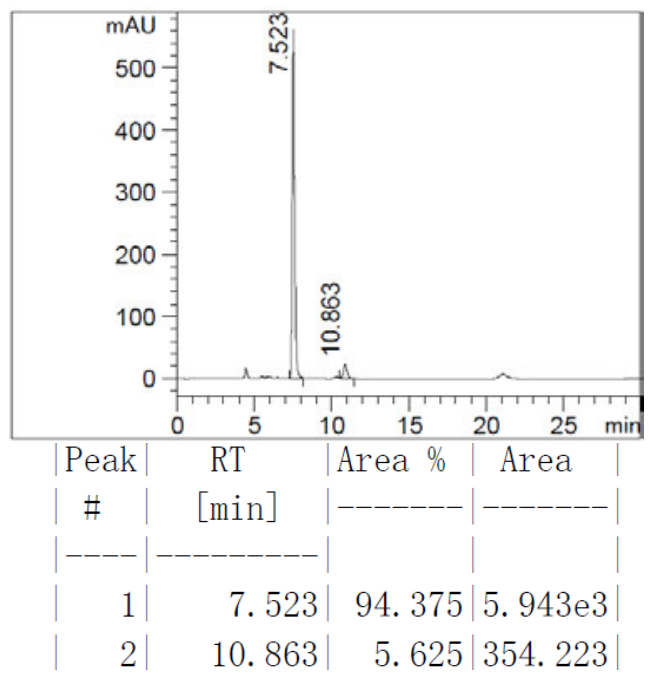


Table 2, entry 17<smiles>c1ccc(C2CC(c3ccsc3)Nc3ccccc32)cc1</smiles>

HPLC Conditions: Column: Chiralcel OD-H, Daicel Chemical Industries, Ltd., Eluent: Hexanes/IPA(90/10 ); Flow rate: $0.8 \mathrm{~mL} / \mathrm{min}$; Detection: UV $254 \mathrm{~nm}$

Racemic

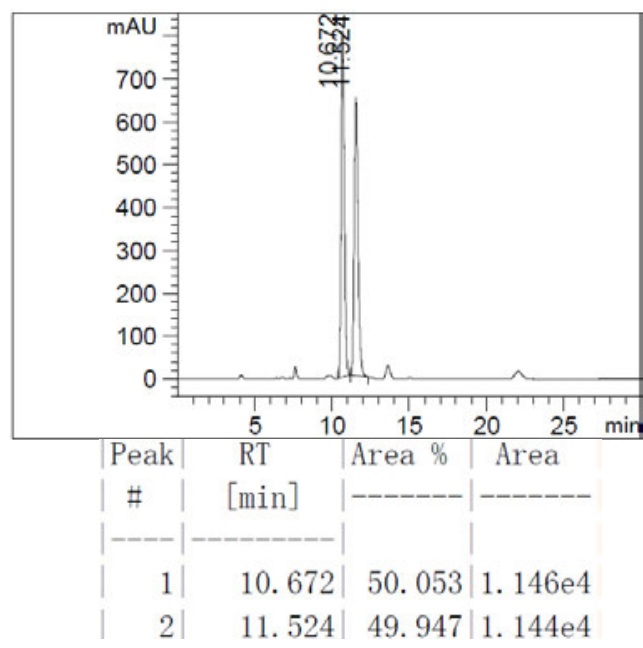

Chiral

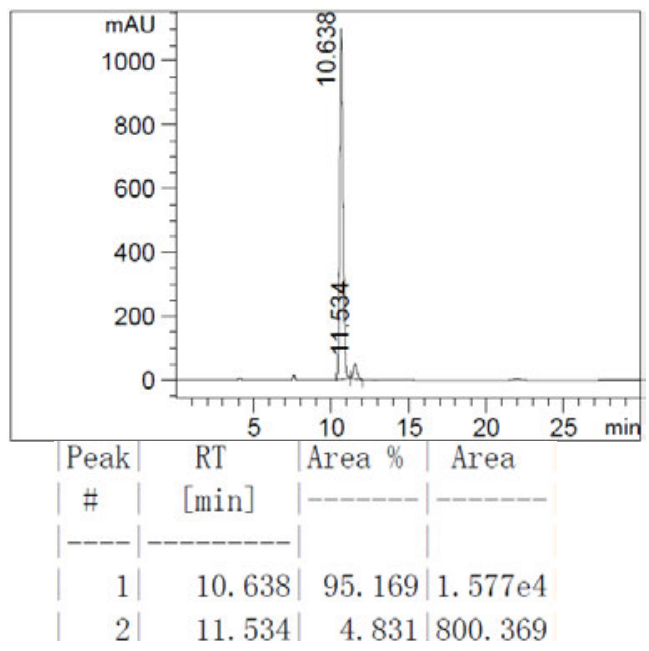

Table 2, entry 18<smiles>Fc1ccc(C2CC(c3ccccc3)Nc3ccccc32)cc1</smiles>

HPLC Conditions: Column: Chiralcel OD-H, Daicel Chemical Industries, Ltd., Eluent: Hexanes/IPA(90/10 ); Flow rate: $0.8 \mathrm{~mL} / \mathrm{min}$; Detection: UV $254 \mathrm{~nm}$

Racemic

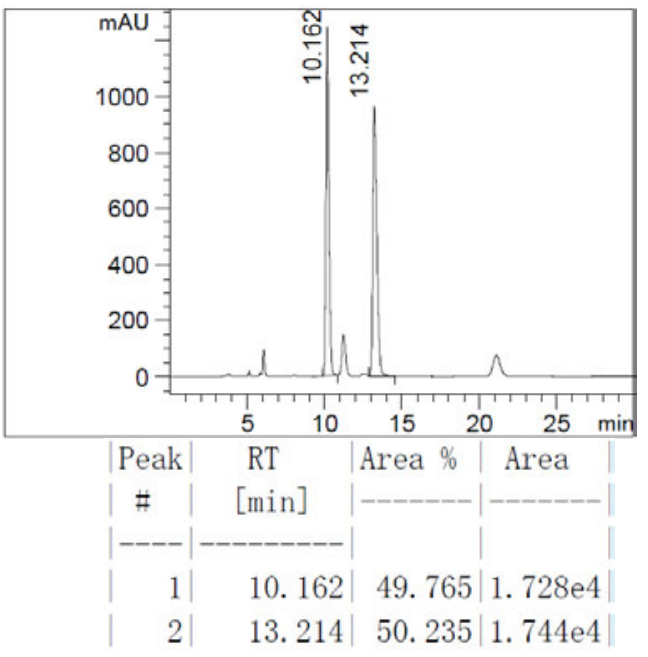

Chiral

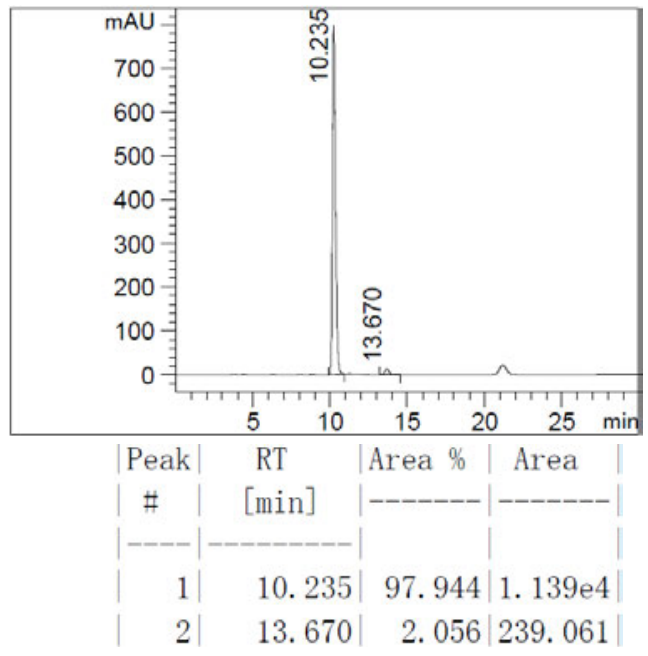


Table 2, entry 19<smiles>Clc1ccc(C2CC(c3ccccc3)Nc3ccccc32)cc1</smiles>

HPLC Conditions: Column: Chiralcel OD-H, Daicel Chemical Industries, Ltd., Eluent: Hexanes/IPA(90/10 ); Flow rate: $0.8 \mathrm{~mL} / \mathrm{min}$; Detection: UV $254 \mathrm{~nm}$
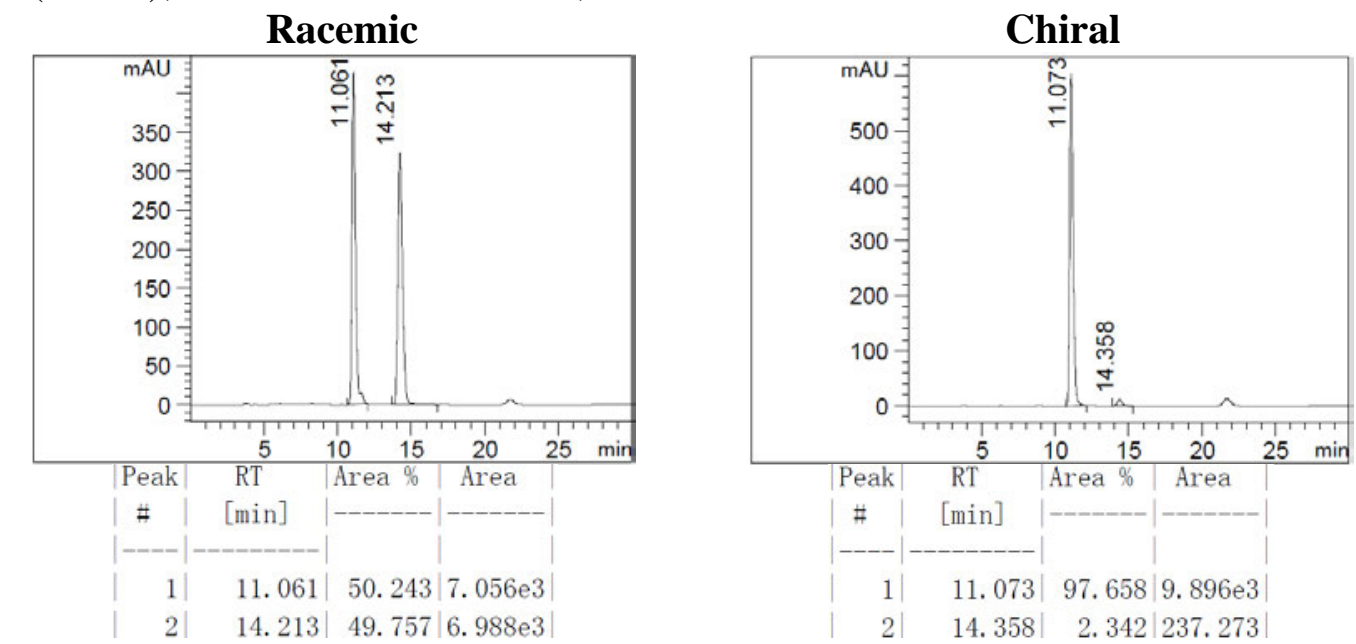

Table 2, entry 20<smiles>Brc1ccc(C2CC(c3ccccc3)Nc3ccccc32)cc1</smiles>

HPLC Conditions: Column: Chiralcel OD-H, Daicel Chemical Industries, Ltd., Eluent: Hexanes/IPA(90/10 ); Flow rate: $0.8 \mathrm{~mL} / \mathrm{min}$; Detection: UV $254 \mathrm{~nm}$

Racemic

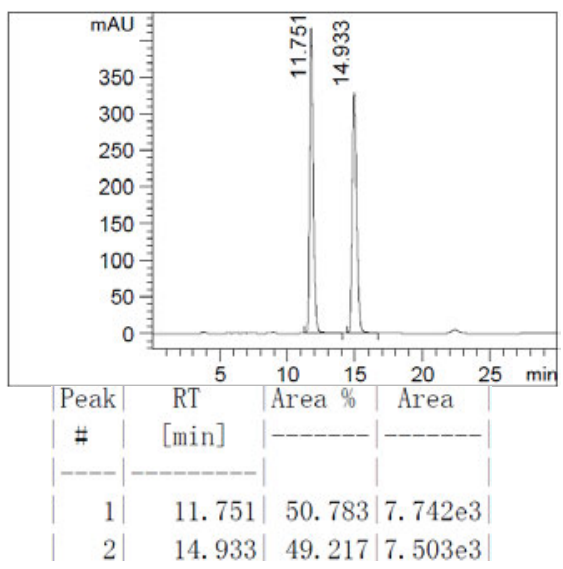

\section{Chiral}

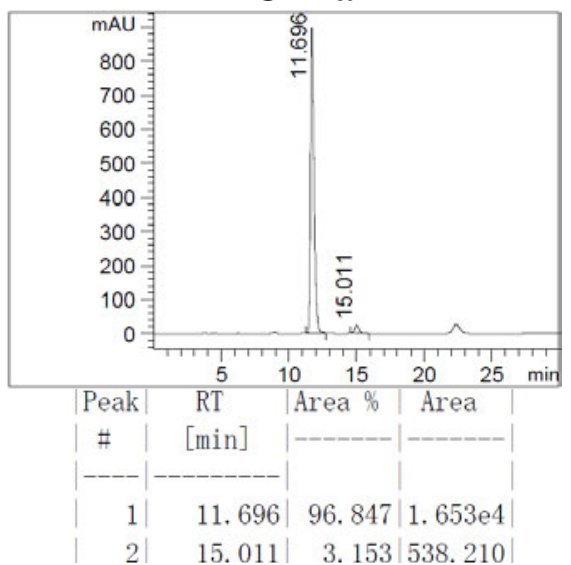


Table 2, entry 21<smiles>Cc1ccc(C2CC(c3ccccc3)Nc3ccccc32)cc1</smiles>

HPLC Conditions: Column: Chiralcel OD-H, Daicel Chemical Industries, Ltd., Eluent: Hexanes/IPA(90/10 ); Flow rate: $0.8 \mathrm{~mL} / \mathrm{min}$; Detection: UV $254 \mathrm{~nm}$
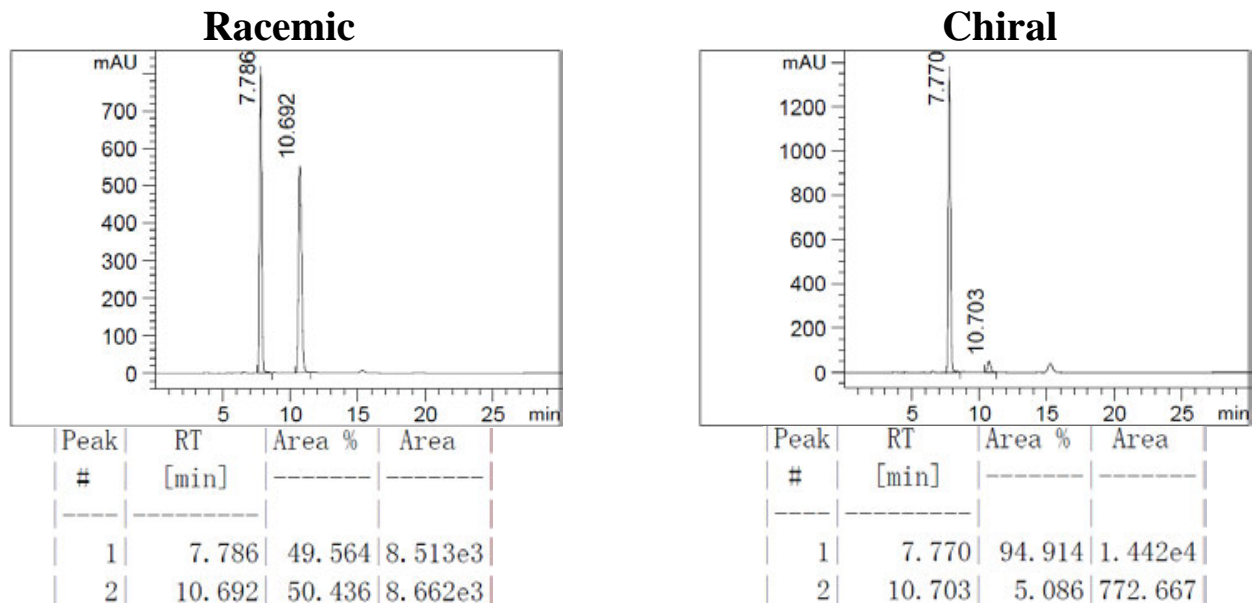

Table 2, entry 22<smiles>COc1ccc(C2CC(c3ccccc3)Nc3ccccc32)cc1</smiles>

HPLC Conditions: Column: Chiralcel OD-H, Daicel Chemical Industries, Ltd., Eluent: Hexanes/IPA(90/10 ); Flow rate: $0.8 \mathrm{~mL} / \mathrm{min}$; Detection: UV $254 \mathrm{~nm}$
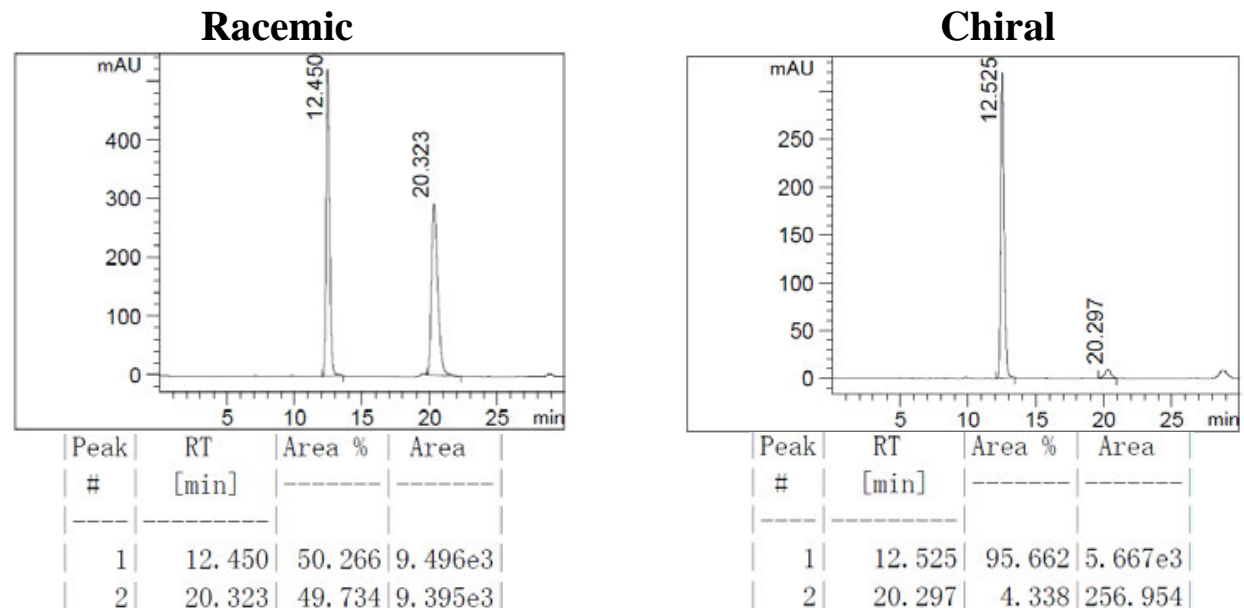
Table 2, entry 23<smiles>C1=C(C2CC(c3ccccc3)Nc3ccccc32)CCCC1</smiles>

HPLC Conditions: Column: Chiralcel OD-H, Daicel Chemical Industries, Ltd., Eluent: Hexanes/IPA(90/10 ); Flow rate: $0.8 \mathrm{~mL} / \mathrm{min}$; Detection: UV $254 \mathrm{~nm}$

Racemic

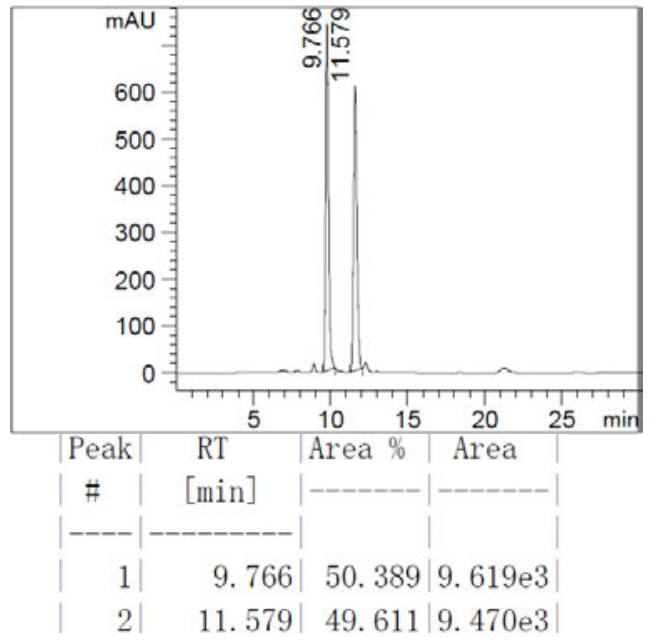

Chiral

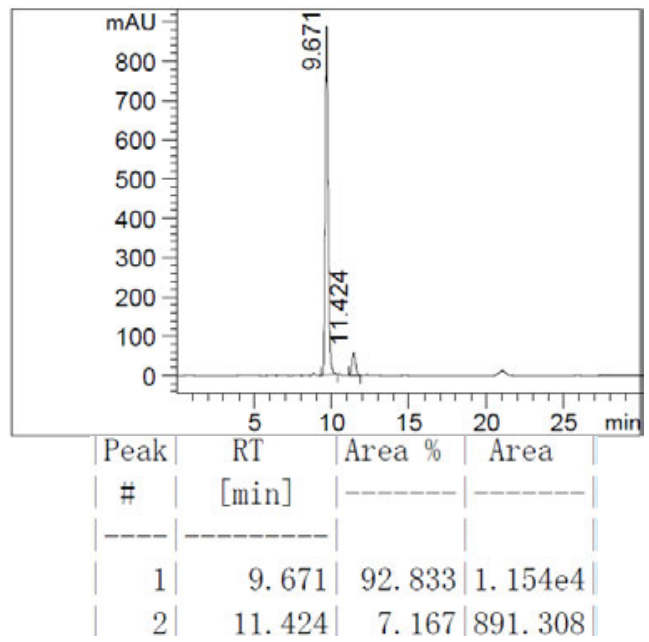

Table 2, entry 24<smiles>Cc1ccc2c(c1)C(c1ccccc1)CC(c1ccccc1)N2</smiles>

HPLC Conditions: Column: Chiralcel OD-H, Daicel Chemical Industries, Ltd., Eluent: Hexanes/IPA(90/10 ); Flow rate: $0.8 \mathrm{~mL} / \mathrm{min}$; Detection: UV $254 \mathrm{~nm}$

Racemic

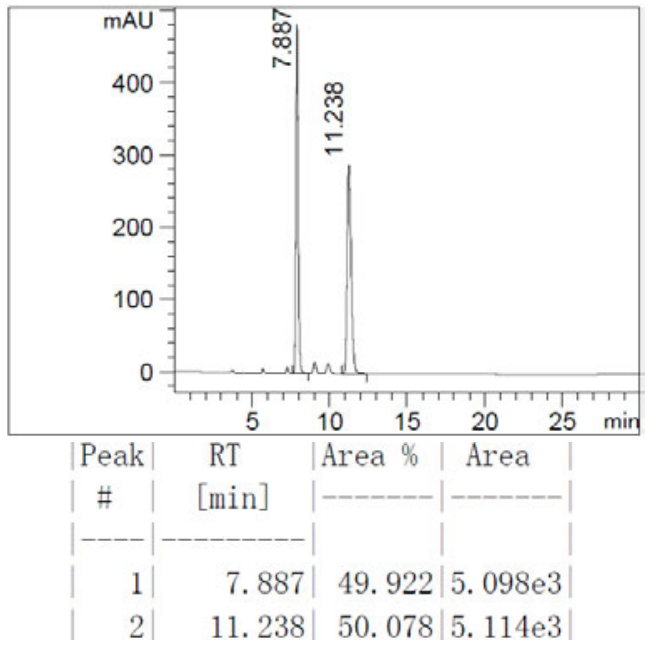

Chiral

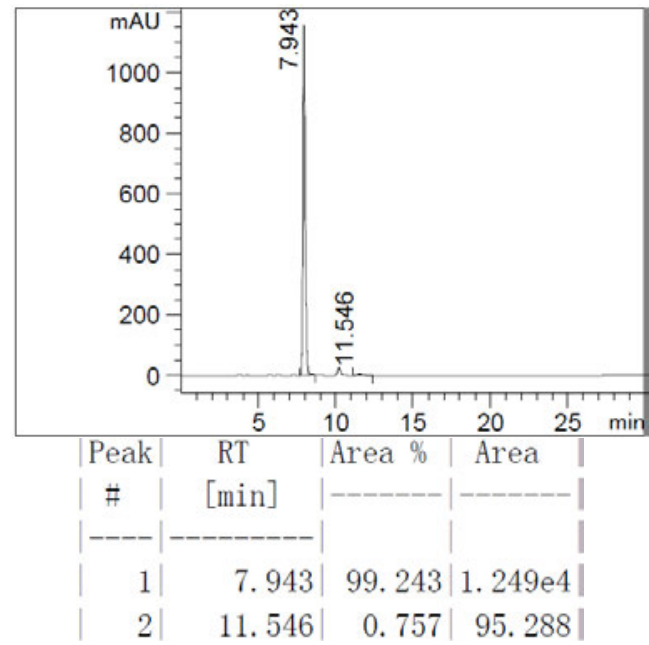


Table 2, entry 25<smiles>Clc1ccc2c(c1)C(c1ccccc1)CC(c1ccccc1)N2</smiles>

HPLC Conditions: Column: Chiralcel OD-H, Daicel Chemical Industries, Ltd., Eluent: Hexanes/IPA(90/10 ); Flow rate: $0.8 \mathrm{~mL} / \mathrm{min}$; Detection: UV $254 \mathrm{~nm}$

\section{Racemic}

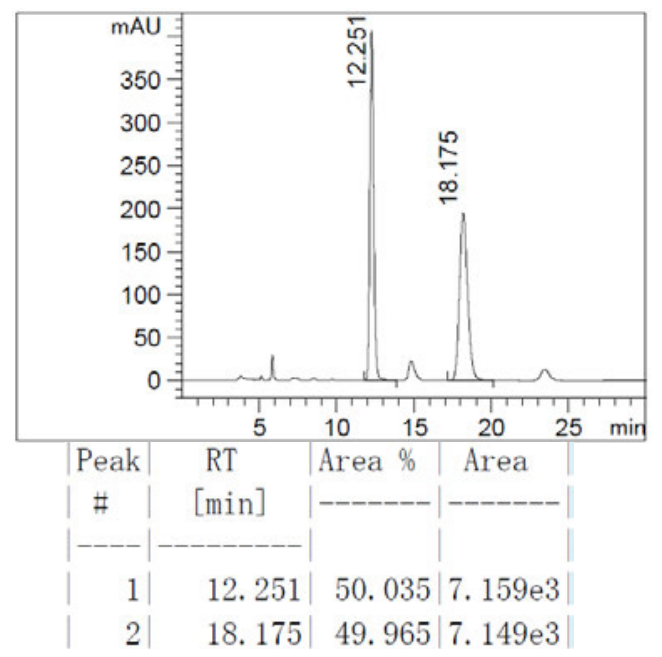

Chiral

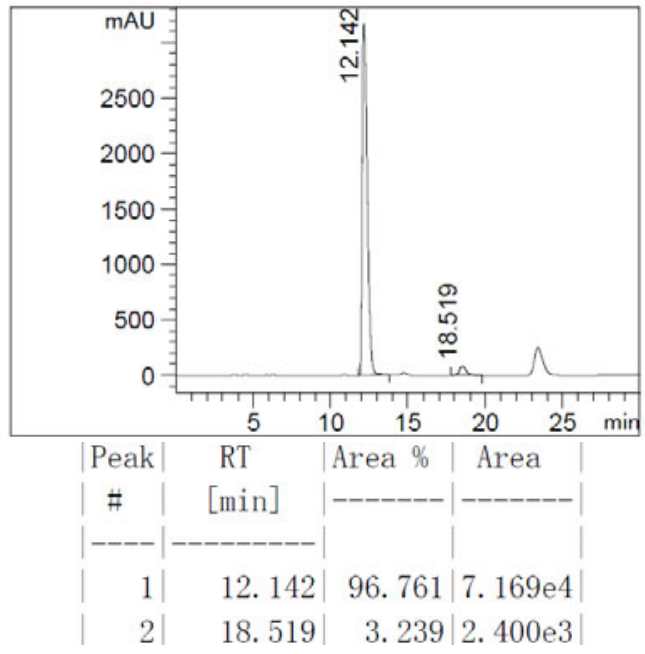

Table 3, entry 1<smiles>CC1Cc2ccccc2NC1c1ccccc1</smiles>

HPLC Conditions: Column: Chiralcel OD-H, Daicel Chemical Industries, Ltd., Eluent: Hexanes/IPA(90/10 ); Flow rate: $0.8 \mathrm{~mL} / \mathrm{min}$; Detection: UV $254 \mathrm{~nm}$

Racemic

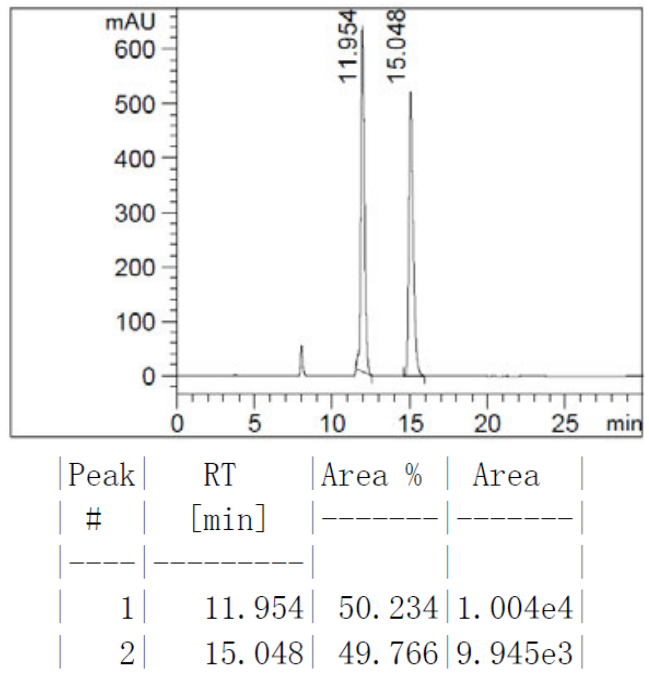

Chiral

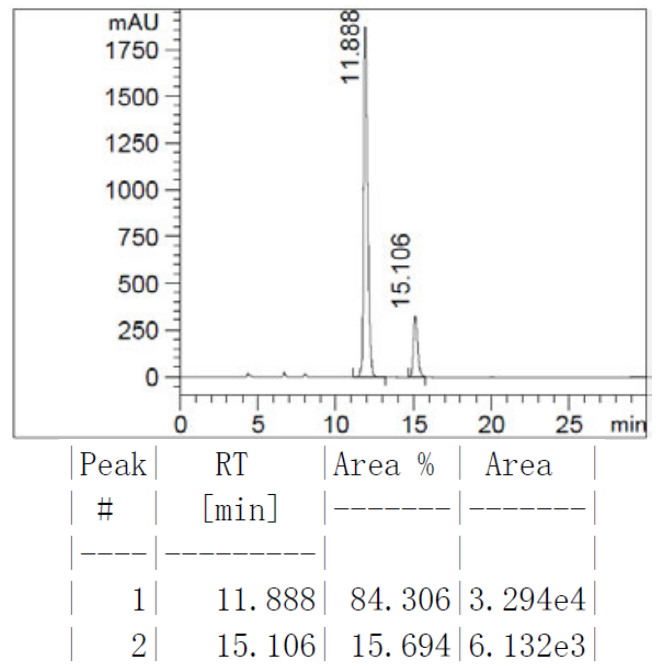


Table 3, entry 2<smiles>CC1Cc2ccccc2NC1c1cccc(Cl)c1</smiles>

HPLC Conditions: Column: Chiralcel OD-H, Daicel Chemical Industries, Ltd., Eluent: Hexanes/IPA(90/10 ); Flow rate: $0.8 \mathrm{~mL} / \mathrm{min}$; Detection: UV $254 \mathrm{~nm}$

Racemic

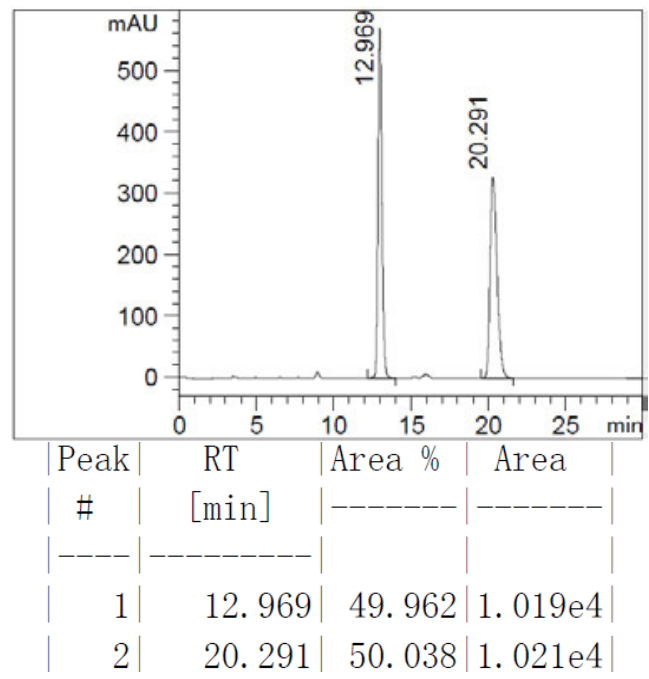

Chiral

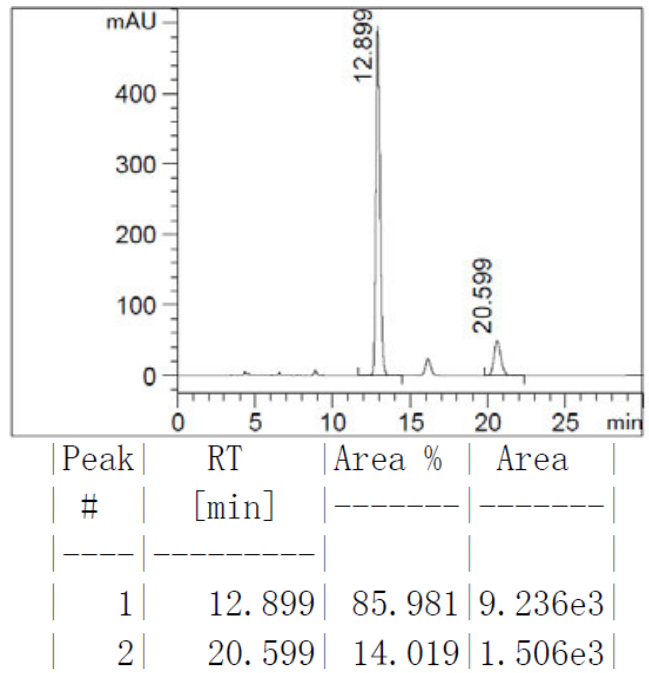

Table 3, entry 3<smiles>CC1Cc2ccccc2NC1c1cccc(Br)c1</smiles>

HPLC Conditions: Column: Chiralcel OD-H, Daicel Chemical Industries, Ltd., Eluent: Hexanes/IPA(90/10 ); Flow rate: $0.8 \mathrm{~mL} / \mathrm{min}$; Detection: UV $254 \mathrm{~nm}$

Racemic

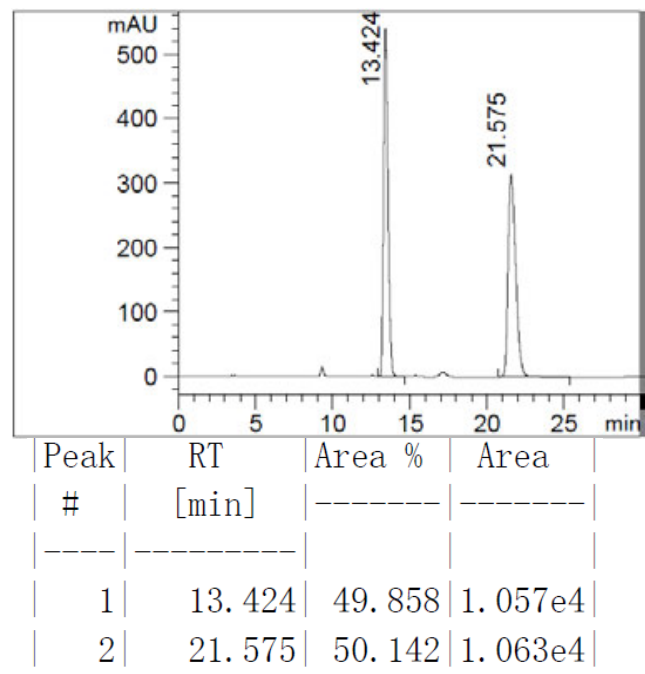

Chiral

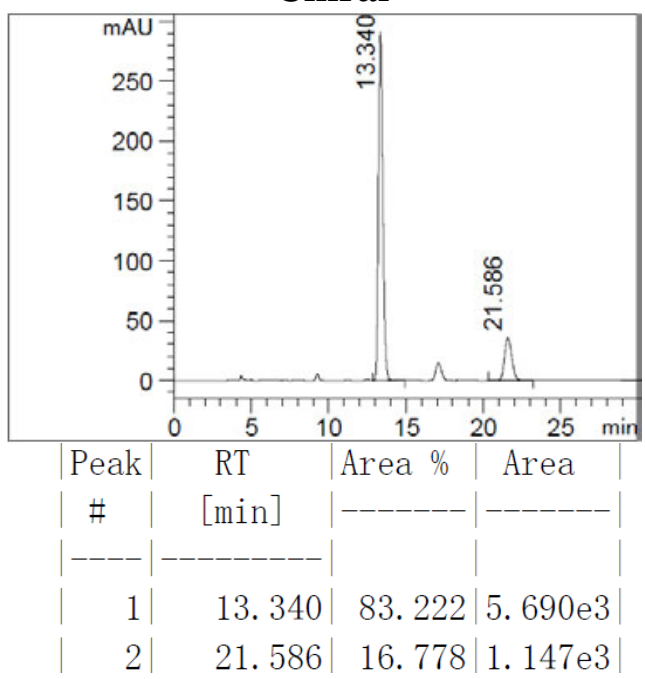


Table 3, entry 4<smiles>CC1Cc2ccccc2NC1c1cccc(C(F)(F)F)c1</smiles>

HPLC Conditions: Column: Chiralcel OD-H, Daicel Chemical Industries, Ltd., Eluent: Hexanes/IPA(90/10 ); Flow rate: $0.8 \mathrm{~mL} / \mathrm{min}$; Detection: UV $254 \mathrm{~nm}$

Racemic

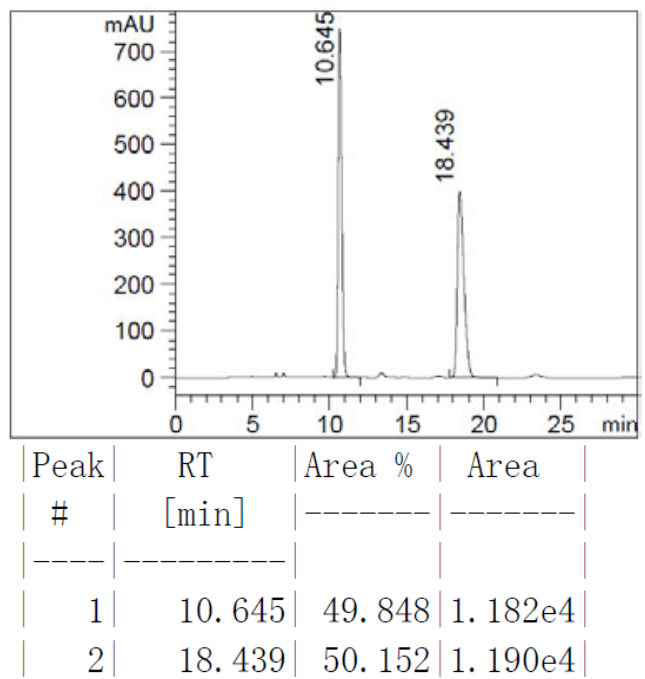

\section{Chiral}

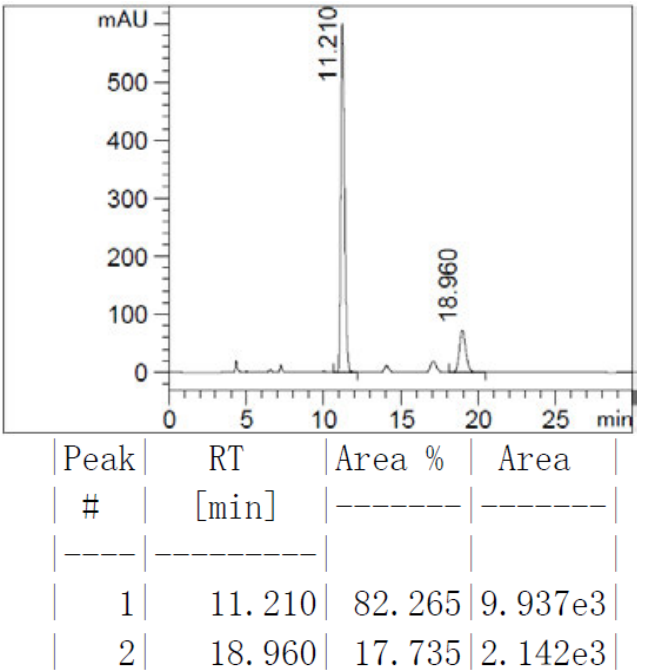

Table 3, entry 5<smiles>Cc1cccc(C2Nc3ccccc3CC2C)c1</smiles>

HPLC Conditions: Column: Chiralcel OD-H, Daicel Chemical Industries, Ltd., Eluent: Hexanes/IPA(90/10 ); Flow rate: $0.8 \mathrm{~mL} / \mathrm{min}$; Detection: UV $254 \mathrm{~nm}$

Racemic

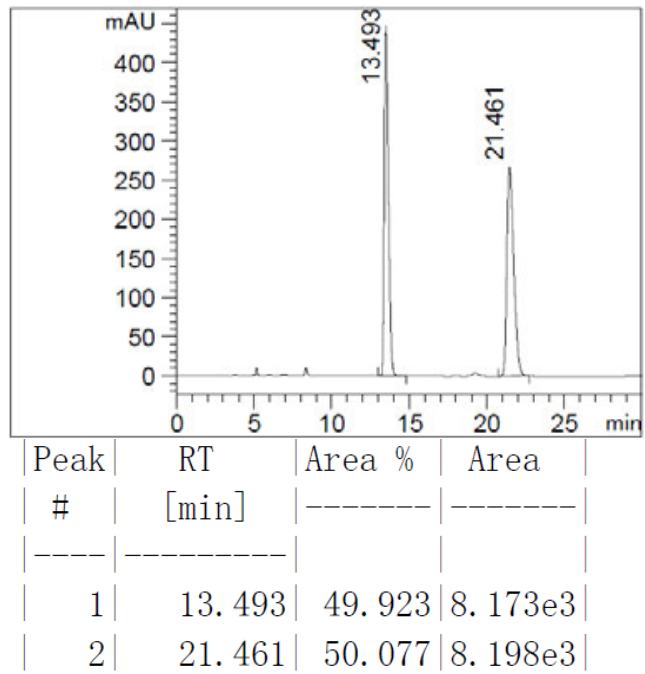

Chiral

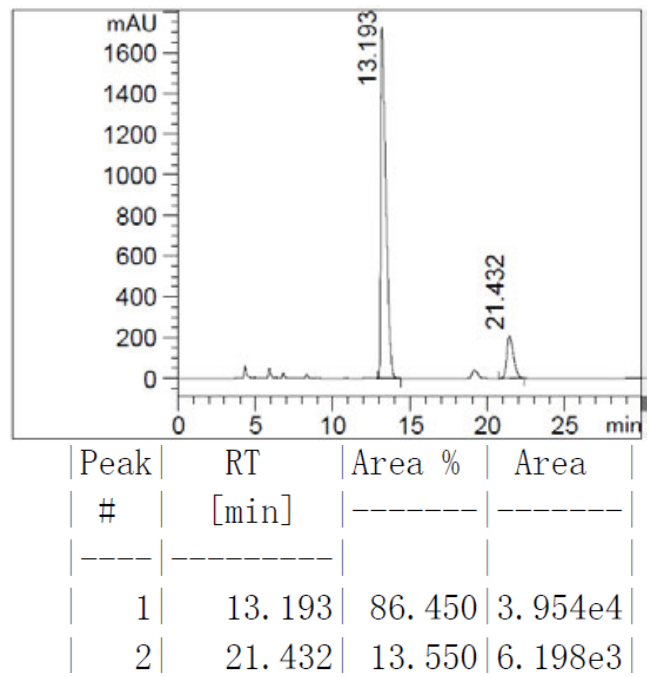


Table 3, entry 6<smiles>CC1Cc2ccccc2NC1c1ccc(Cl)cc1</smiles>

HPLC Conditions: Column: Chiralcel OJ-H, Daicel Chemical Industries, Ltd., Eluent: Hexanes/IPA(90/10 ); Flow rate: $0.8 \mathrm{~mL} / \mathrm{min}$; Detection: UV $254 \mathrm{~nm}$

Racemic

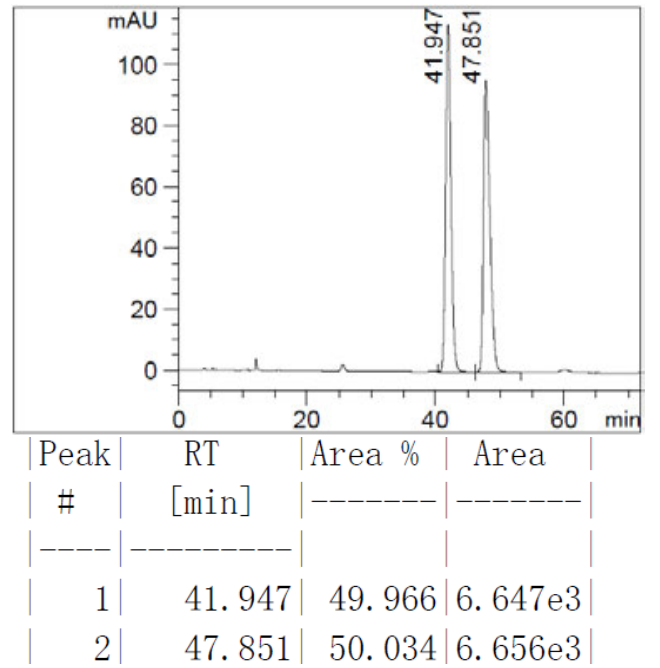

\section{Chiral}

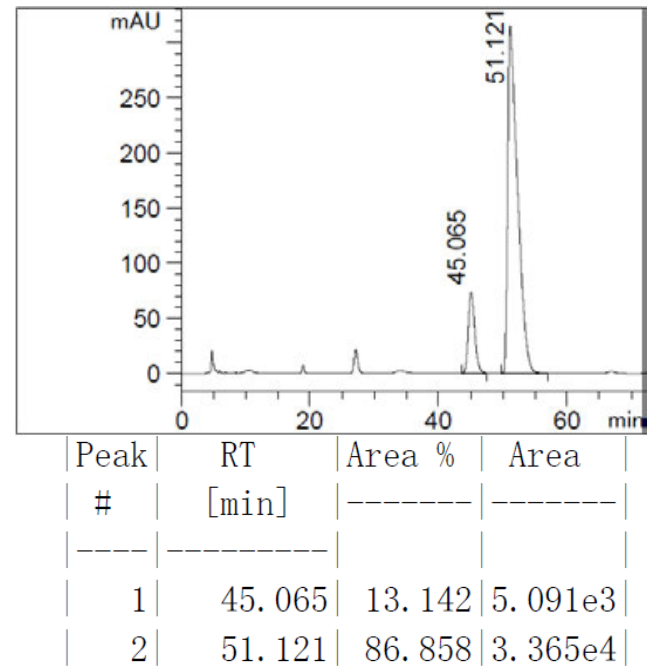

Table 3, entry 7<smiles>CC1Cc2ccccc2NC1c1ccc(Br)cc1</smiles>

HPLC Conditions: Column: Chiralcel OD-H, Daicel Chemical Industries, Ltd., Eluent: Hexanes/IPA(90/10 ); Flow rate: $0.8 \mathrm{~mL} / \mathrm{min}$; Detection: UV $254 \mathrm{~nm}$

Racemic

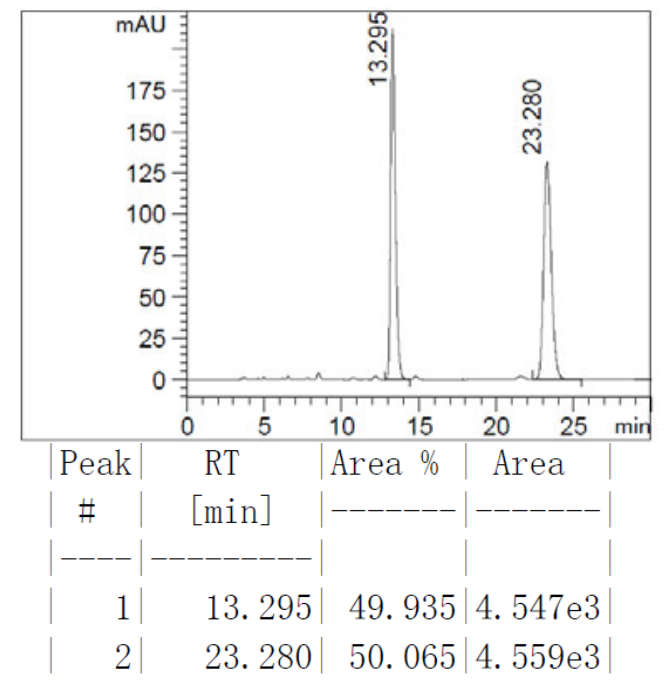

Chiral

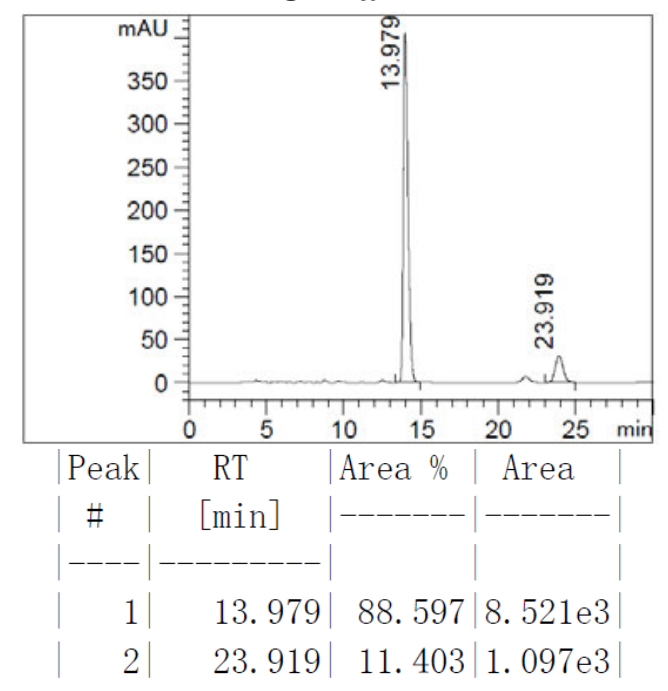


Table 3, entry 8<smiles>CC1Cc2ccccc2NC1c1ccc(C(F)(F)F)cc1</smiles>

HPLC Conditions: Column: Chiralcel OD-H, Daicel Chemical Industries, Ltd., Eluent: Hexanes/IPA(90/10 ); Flow rate: $0.8 \mathrm{~mL} / \mathrm{min}$; Detection: UV $254 \mathrm{~nm}$

Racemic

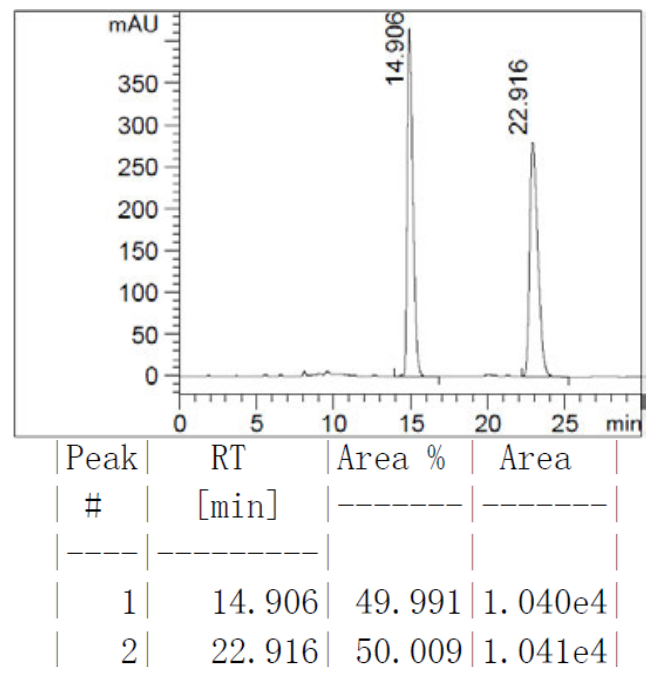

\section{Chiral}

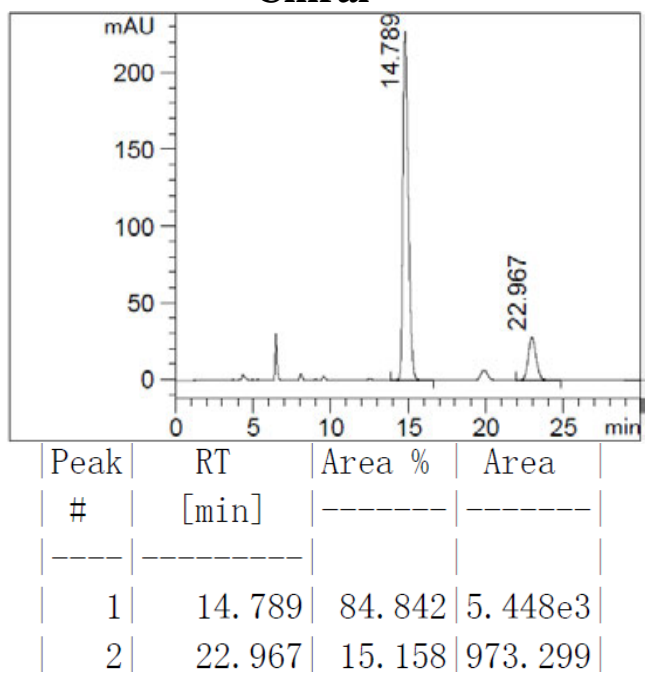

Table 3, entry 9<smiles>Cc1ccc(C2Nc3ccccc3CC2C)cc1</smiles>

HPLC Conditions: Column: Chiralcel OD-H, Daicel Chemical Industries, Ltd., Eluent: Hexanes/IPA(90/10 ); Flow rate: $0.8 \mathrm{~mL} / \mathrm{min}$; Detection: UV $254 \mathrm{~nm}$

Racemic

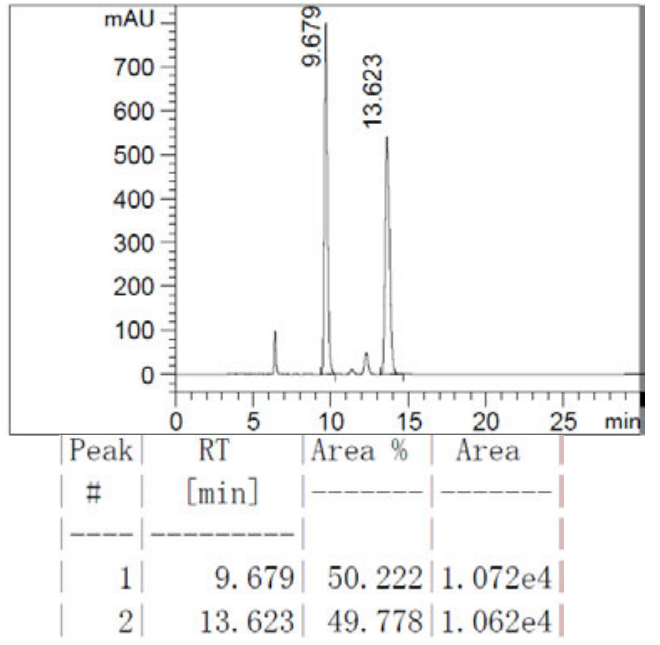

Chiral

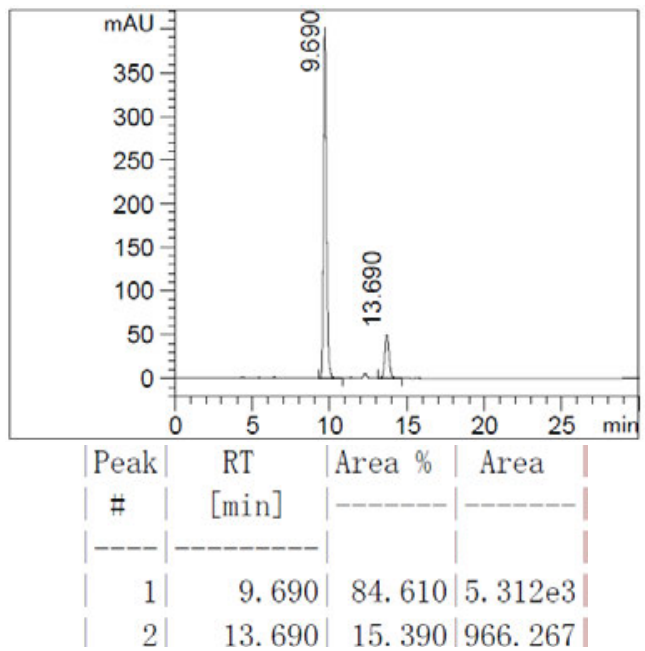


Table 3, entry 10<smiles>[CH]C1Cc2ccccc2NC1c1ccc(OC)cc1</smiles>

HPLC Conditions: Column: Chiralcel OD-H, Daicel Chemical Industries, Ltd., Eluent: Hexanes/IPA(90/10 ); Flow rate: $0.8 \mathrm{~mL} / \mathrm{min}$; Detection: UV $254 \mathrm{~nm}$

Racemic

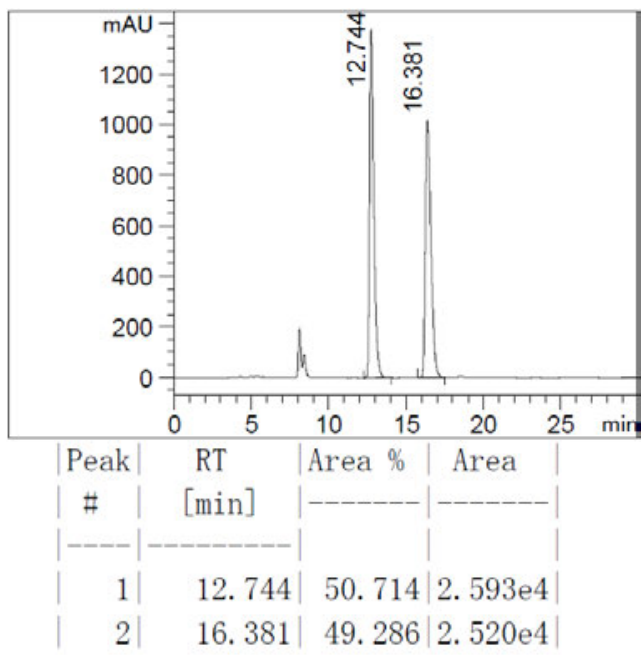

Chiral

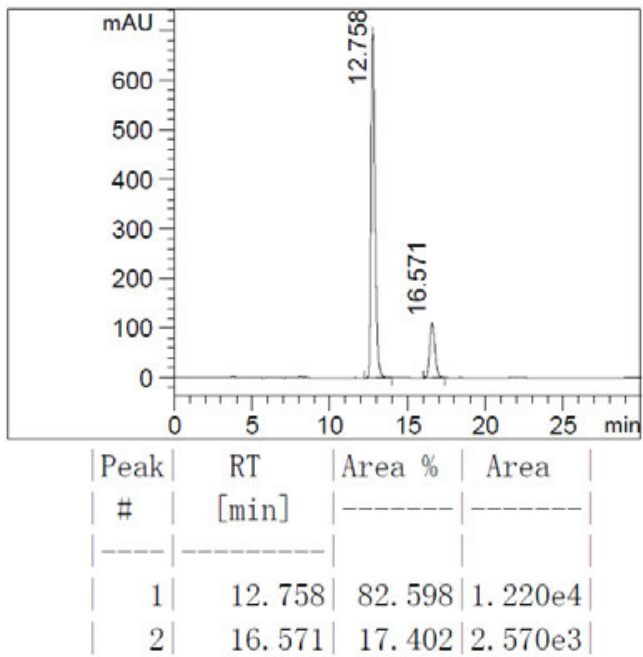

Table 3, entry 11<smiles>CC1Cc2ccccc2NC1c1ccco1</smiles>

HPLC Conditions: Column: Chiralcel OJ-H, Daicel Chemical Industries, Ltd., Eluent: Hexanes/IPA(90/10 ); Flow rate: $0.8 \mathrm{~mL} / \mathrm{min}$; Detection: UV $254 \mathrm{~nm}$

Racemic

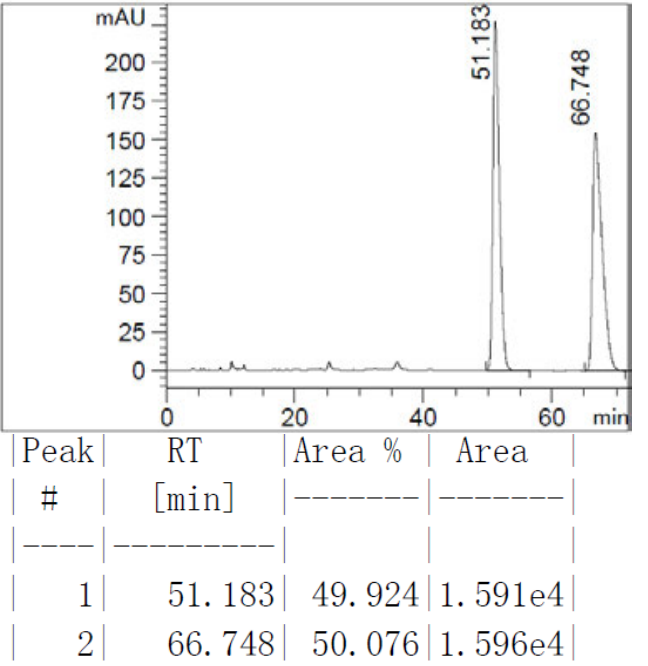

\section{Chiral}

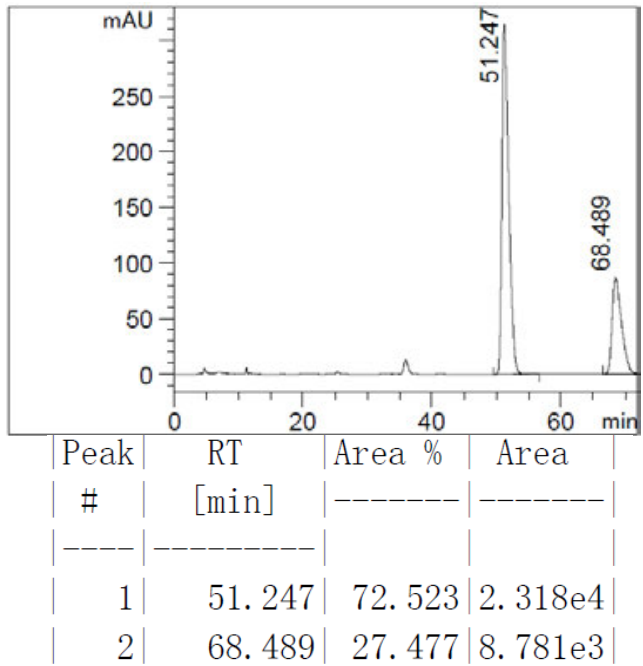


Table 3, entry 12<smiles>CC1Cc2ccccc2NC1c1cccs1</smiles>

HPLC Conditions: Column: Chiralcel OD-H, Daicel Chemical Industries, Ltd., Eluent: Hexanes/IPA(90/10 ); Flow rate: $0.8 \mathrm{~mL} / \mathrm{min}$; Detection: UV $254 \mathrm{~nm}$

Racemic

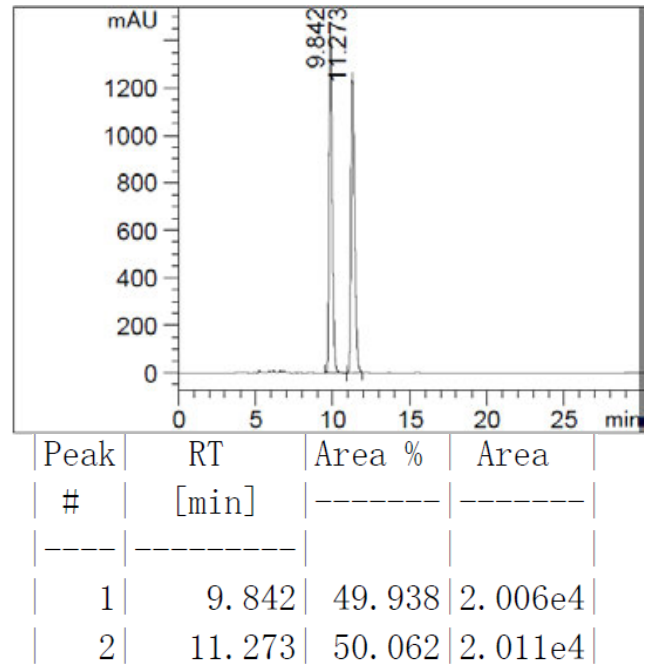

Chiral

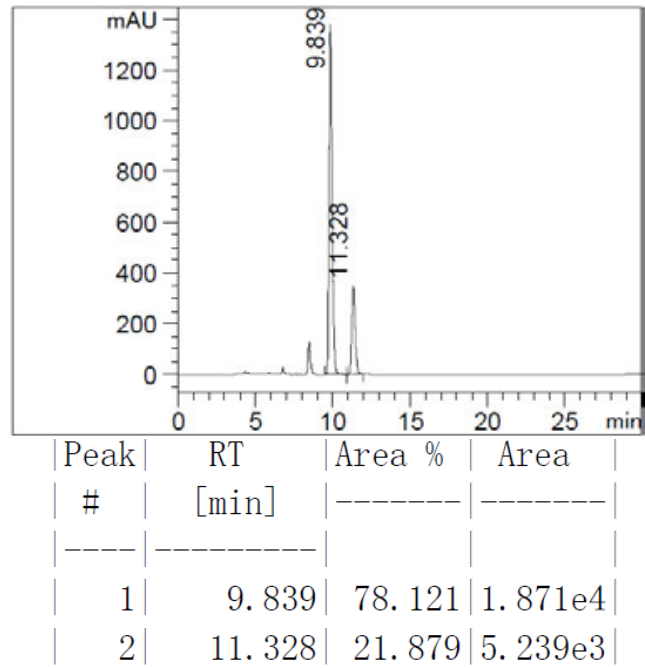

Table 3, entry 13<smiles>CCC1Cc2ccccc2NC1c1ccccc1</smiles>

HPLC Conditions: Column: Chiralcel OD-H, Daicel Chemical Industries, Ltd., Eluent: Hexanes/IPA(90/10 ); Flow rate: $0.8 \mathrm{~mL} / \mathrm{min}$; Detection: UV $254 \mathrm{~nm}$

\section{Racemic}

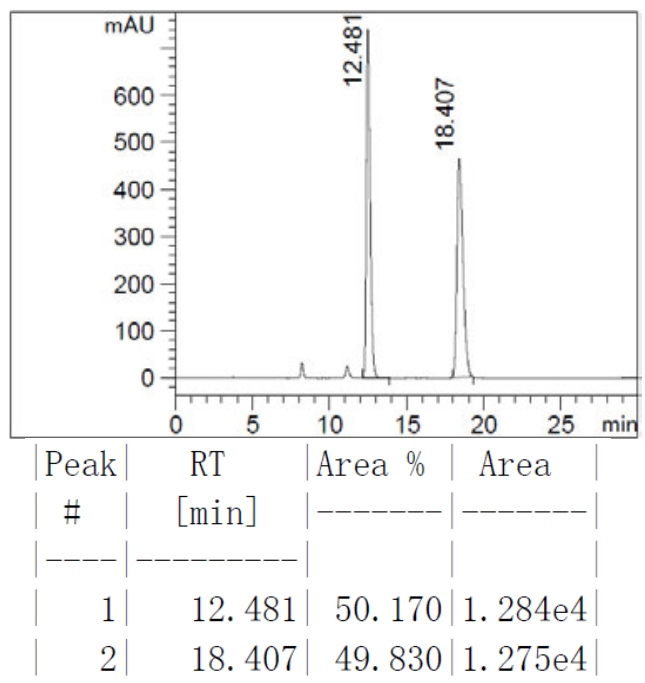

Chiral

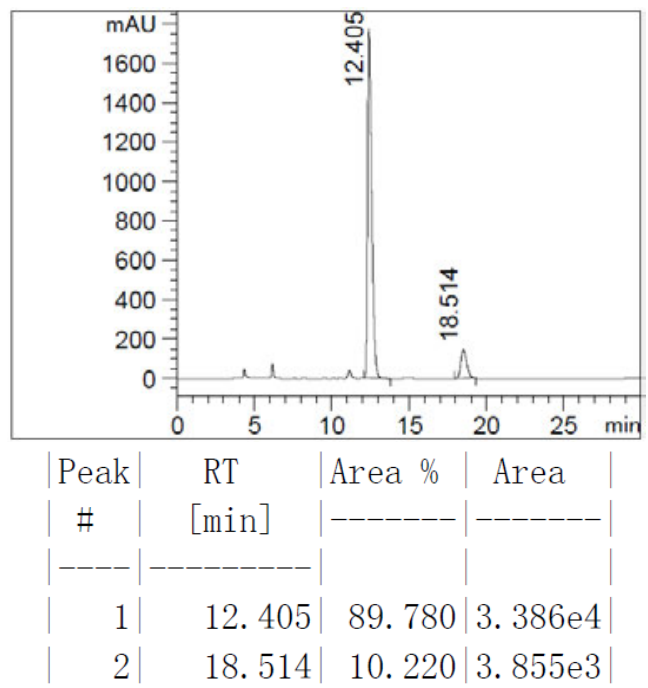


Table 3, entry 14<smiles>CCCCCCCC1Nc2ccccc2CC1c1ccccc1</smiles>

HPLC Conditions: Column: Chiralcel OD-H, Daicel Chemical Industries, Ltd., Eluent: Hexanes/IPA(90/10 ); Flow rate: $0.8 \mathrm{~mL} / \mathrm{min}$; Detection: UV $254 \mathrm{~nm}$

Racemic

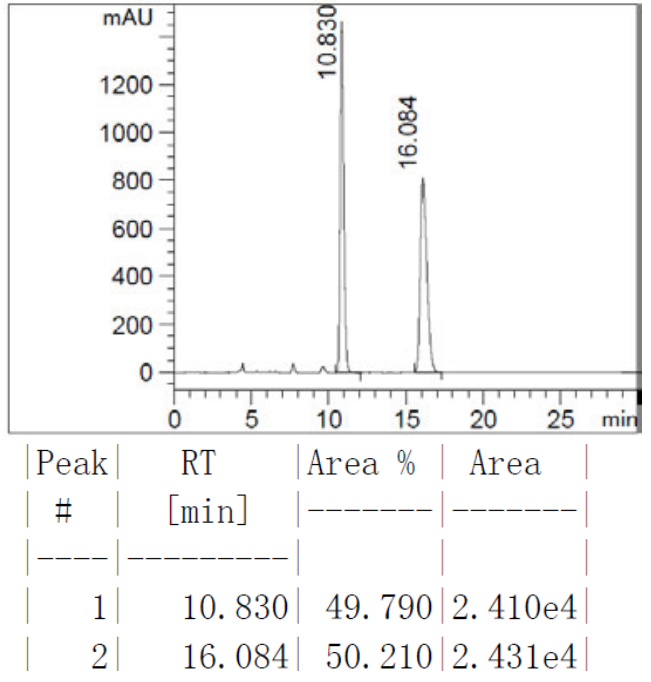

Chiral

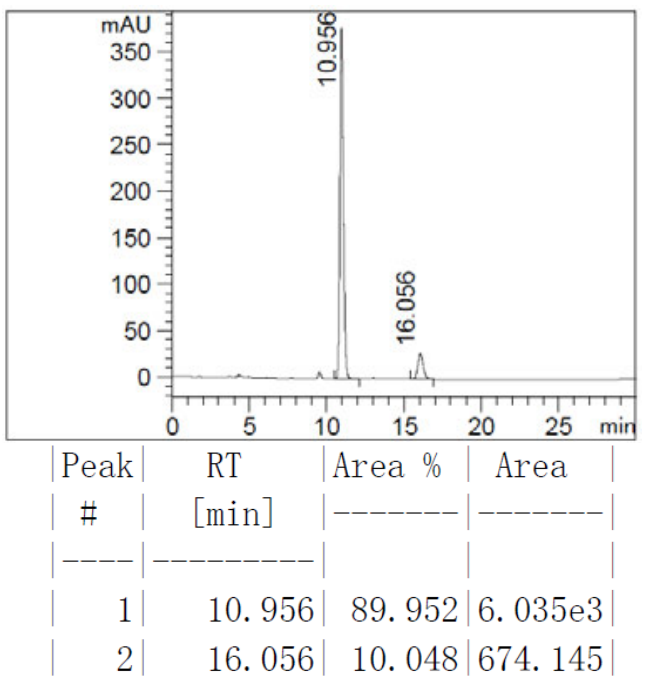

Table 3, entry 15<smiles>CCCCCCC1Cc2ccccc2NC1c1ccccc1</smiles>

HPLC Conditions: Column: Chiralcel OD-H, Daicel Chemical Industries, Ltd., Eluent: Hexanes/IPA(90/10 ); Flow rate: $0.8 \mathrm{~mL} / \mathrm{min}$; Detection: UV $254 \mathrm{~nm}$

Racemic

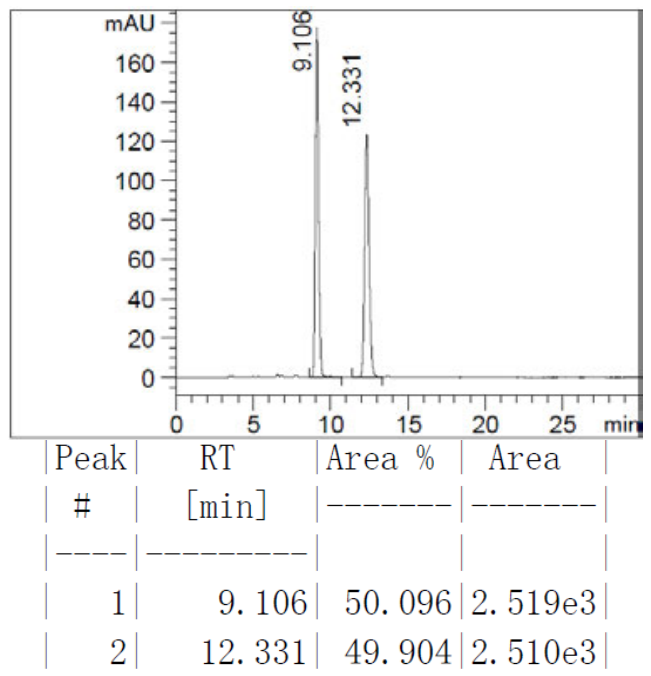

\section{Chiral}

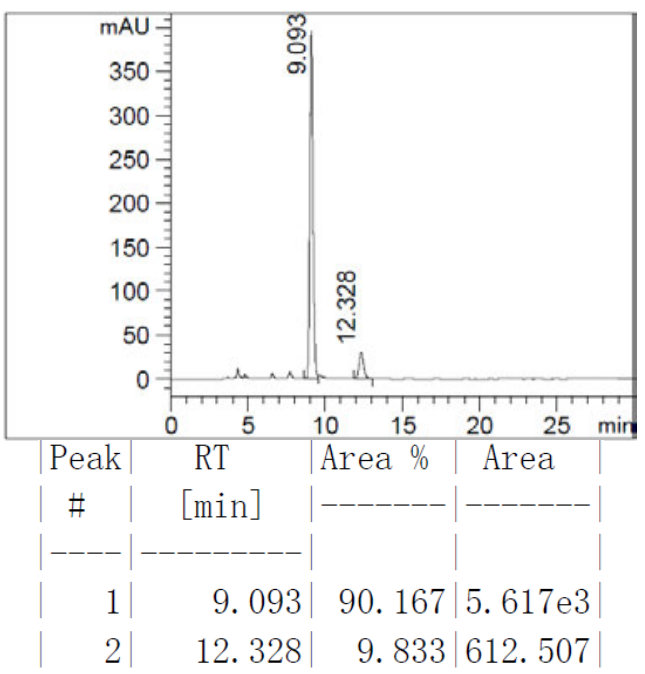


NMR spectra for the substrates and products.

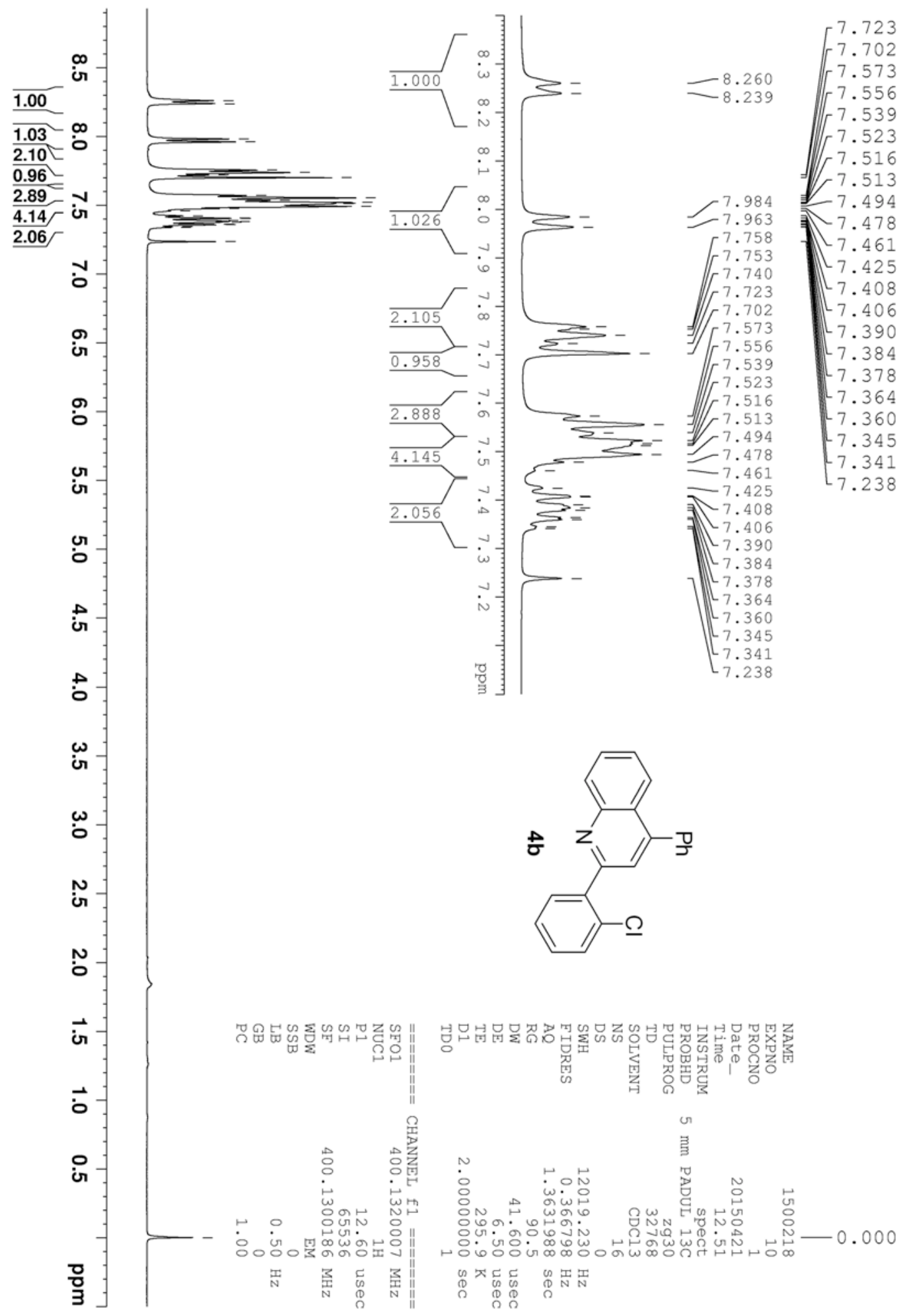




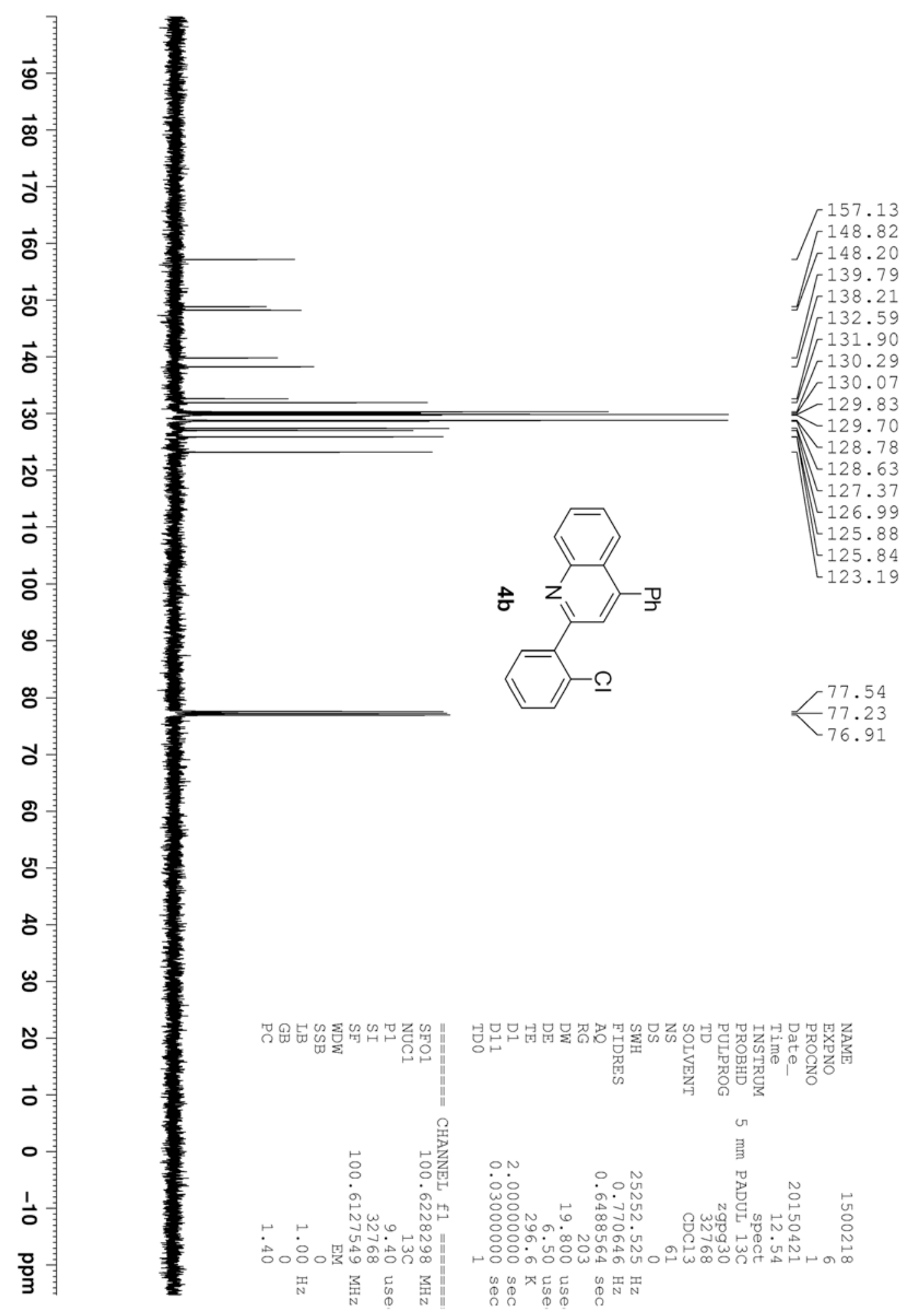




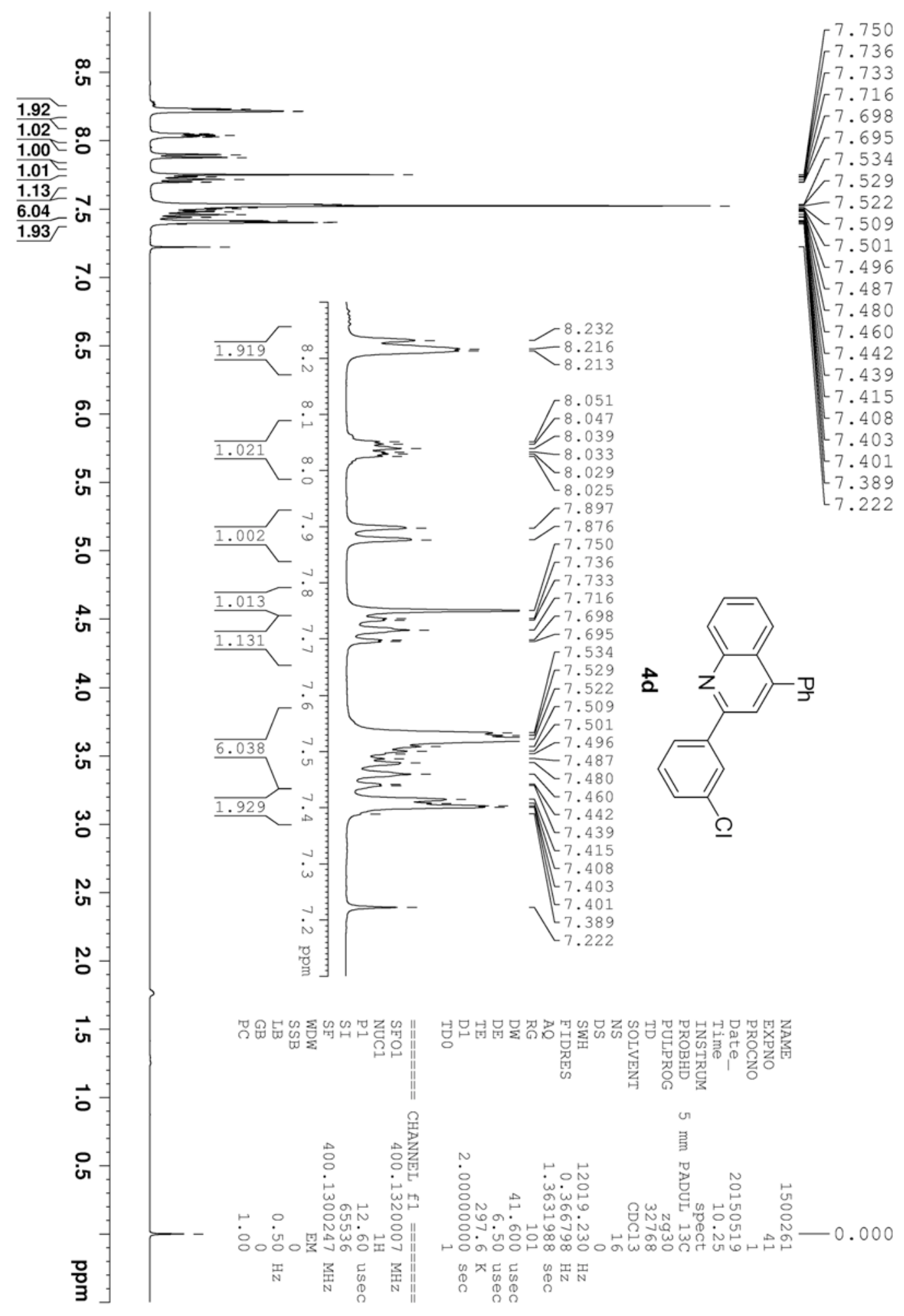




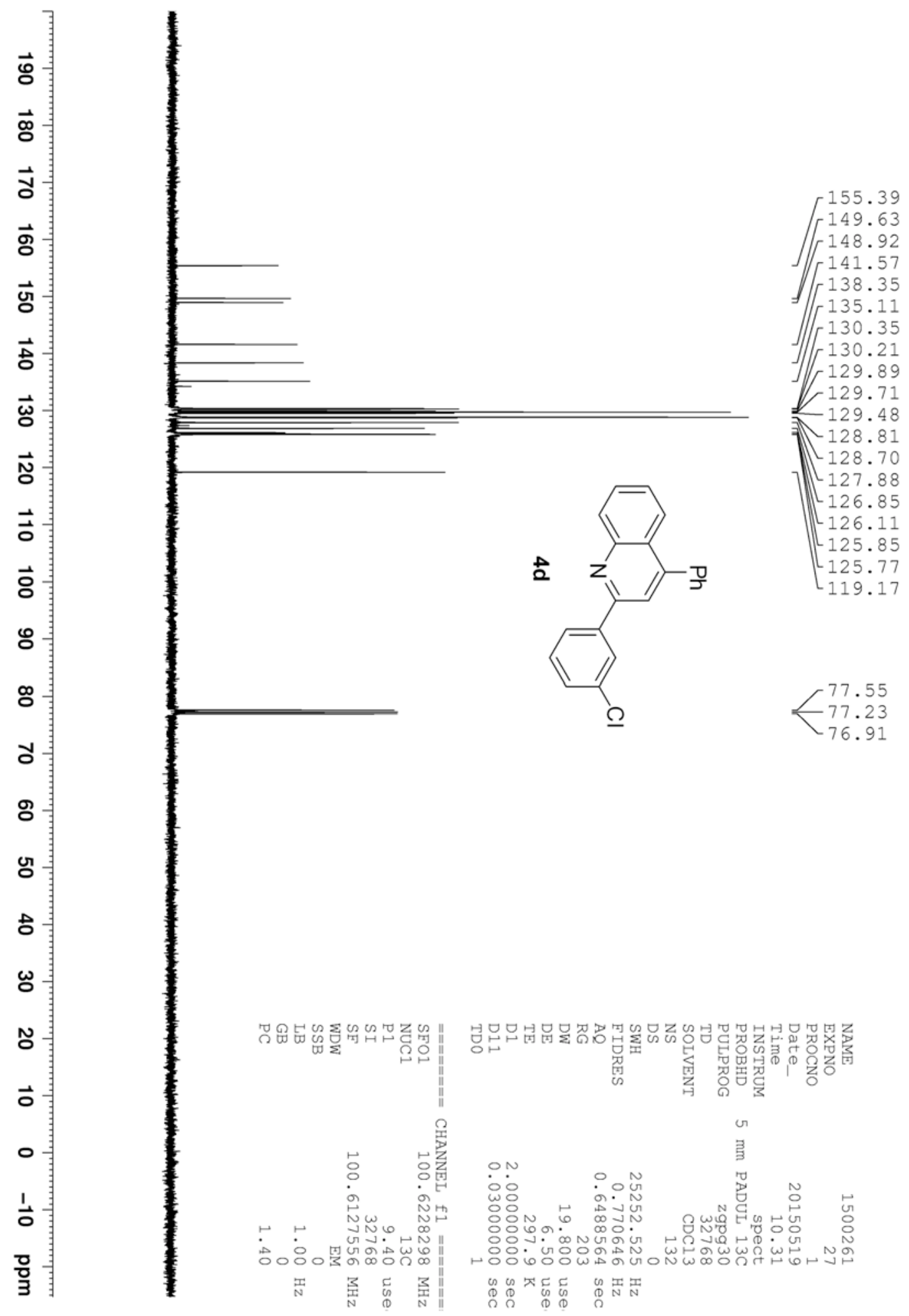




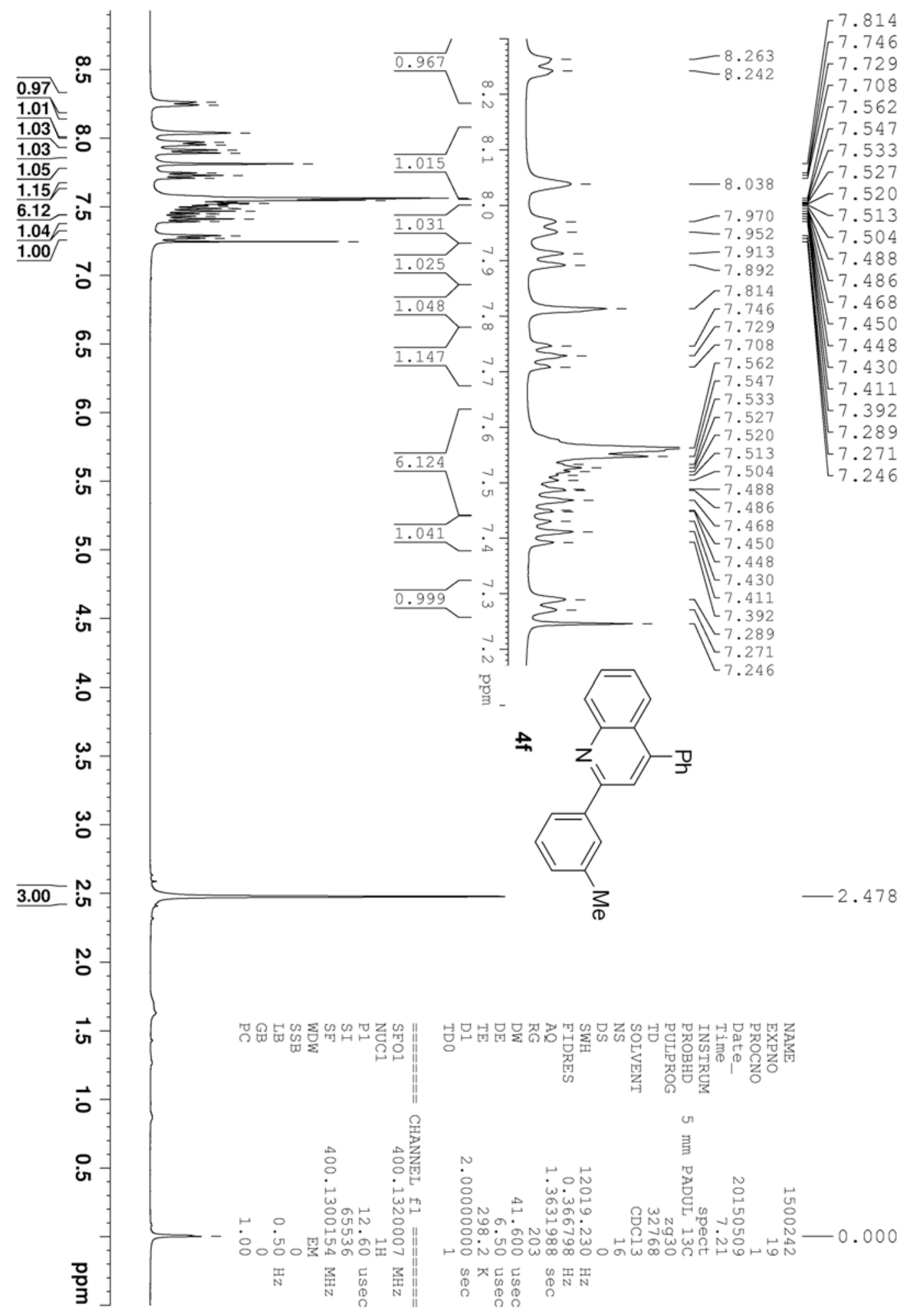




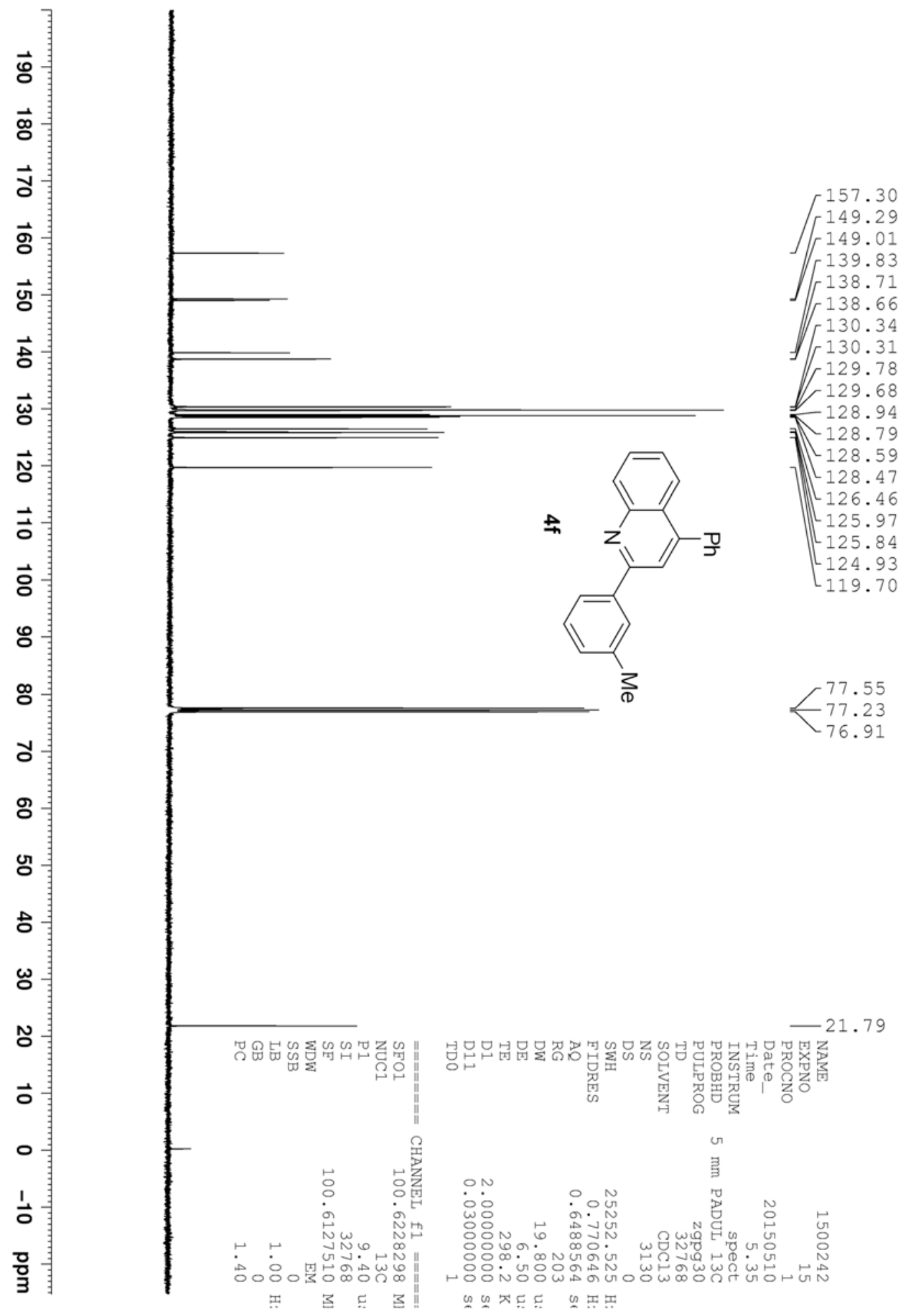




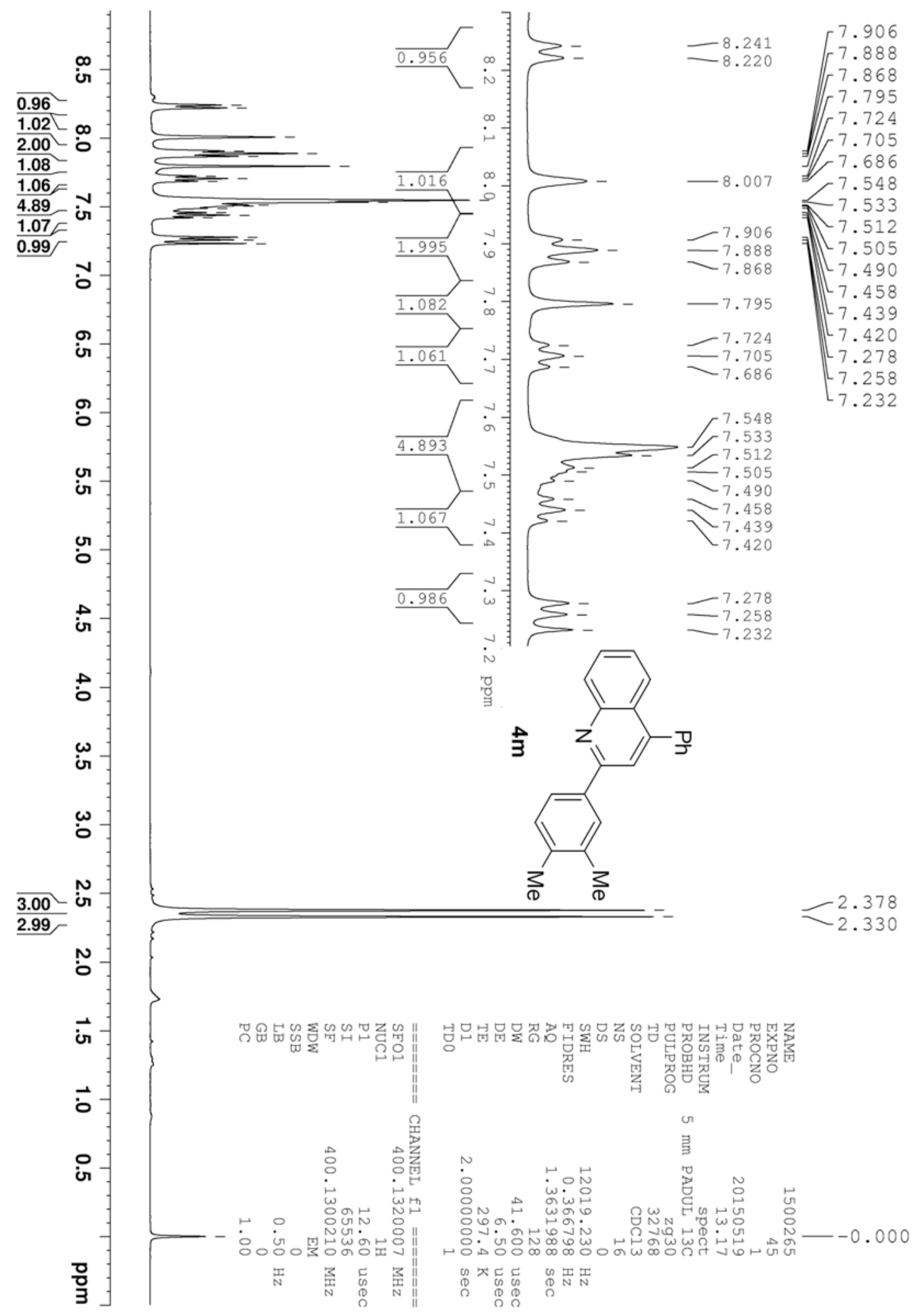




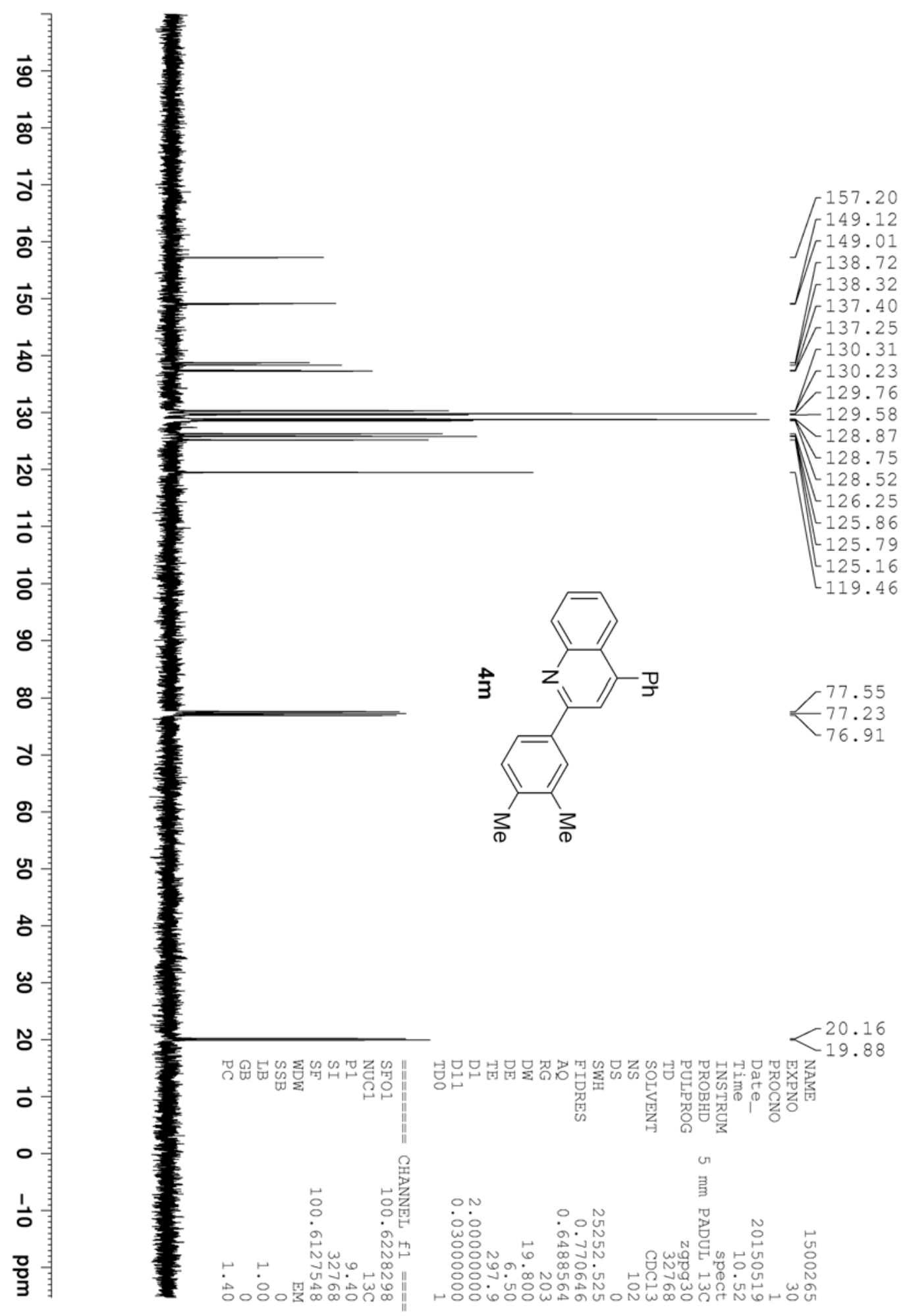




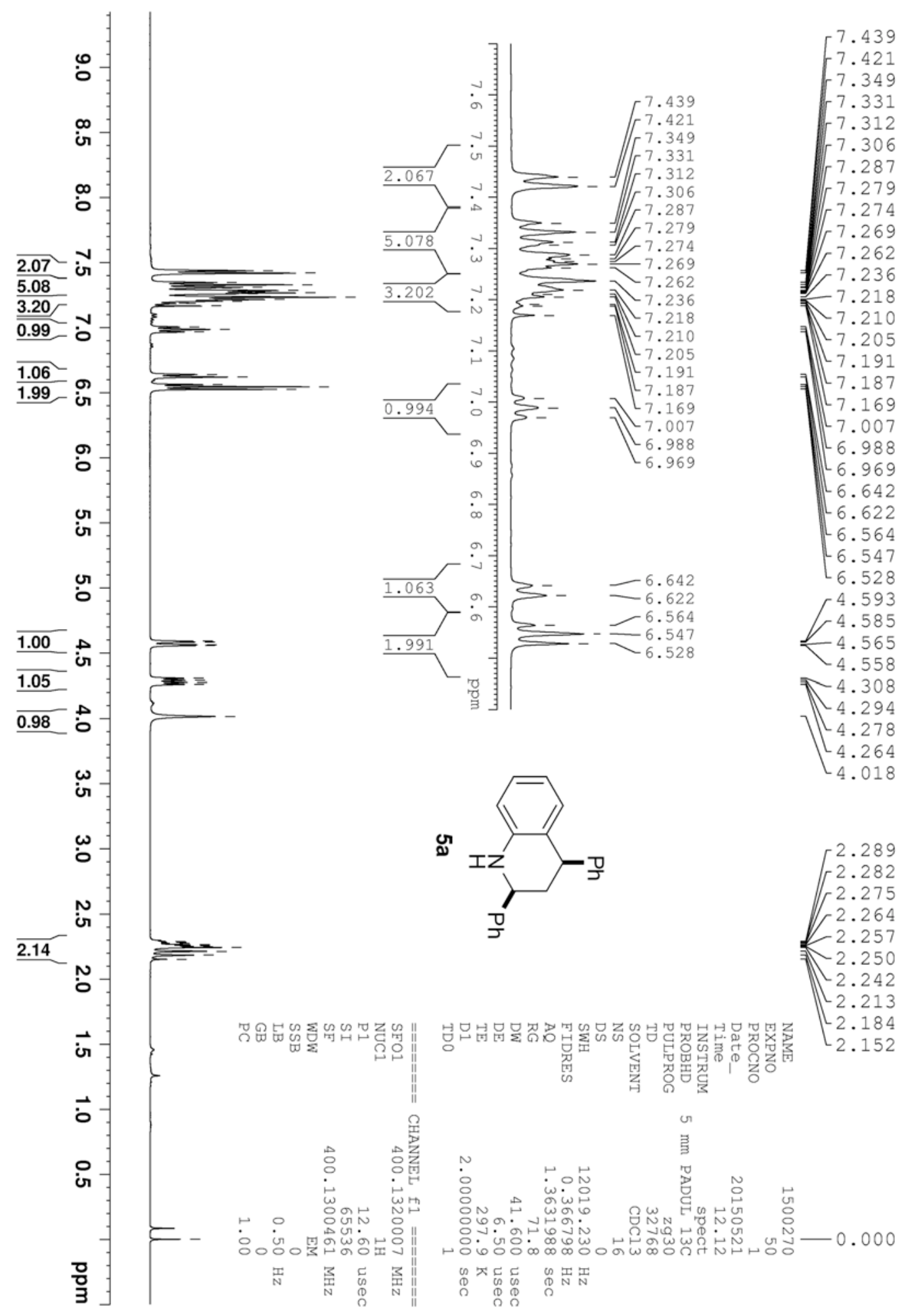




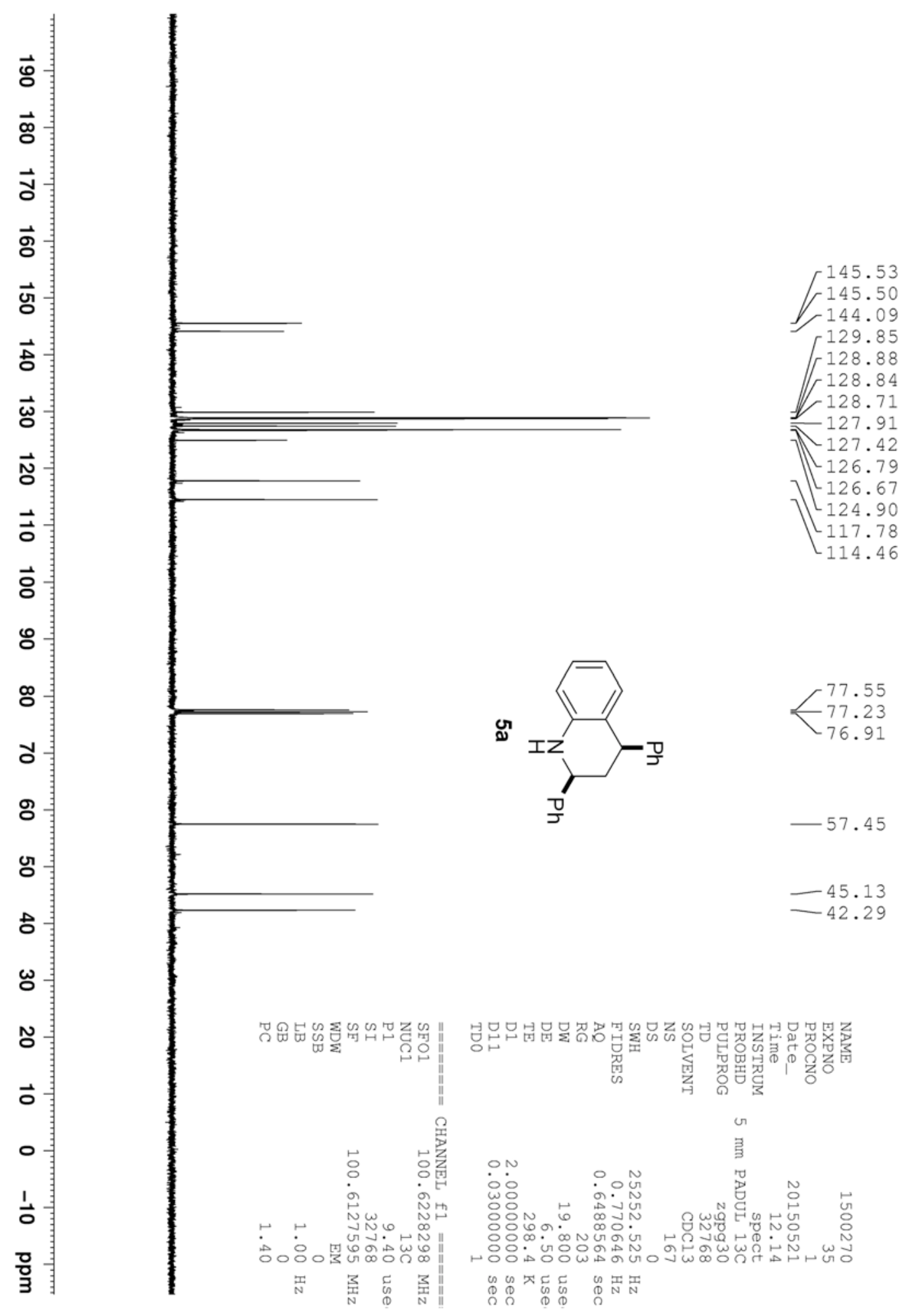




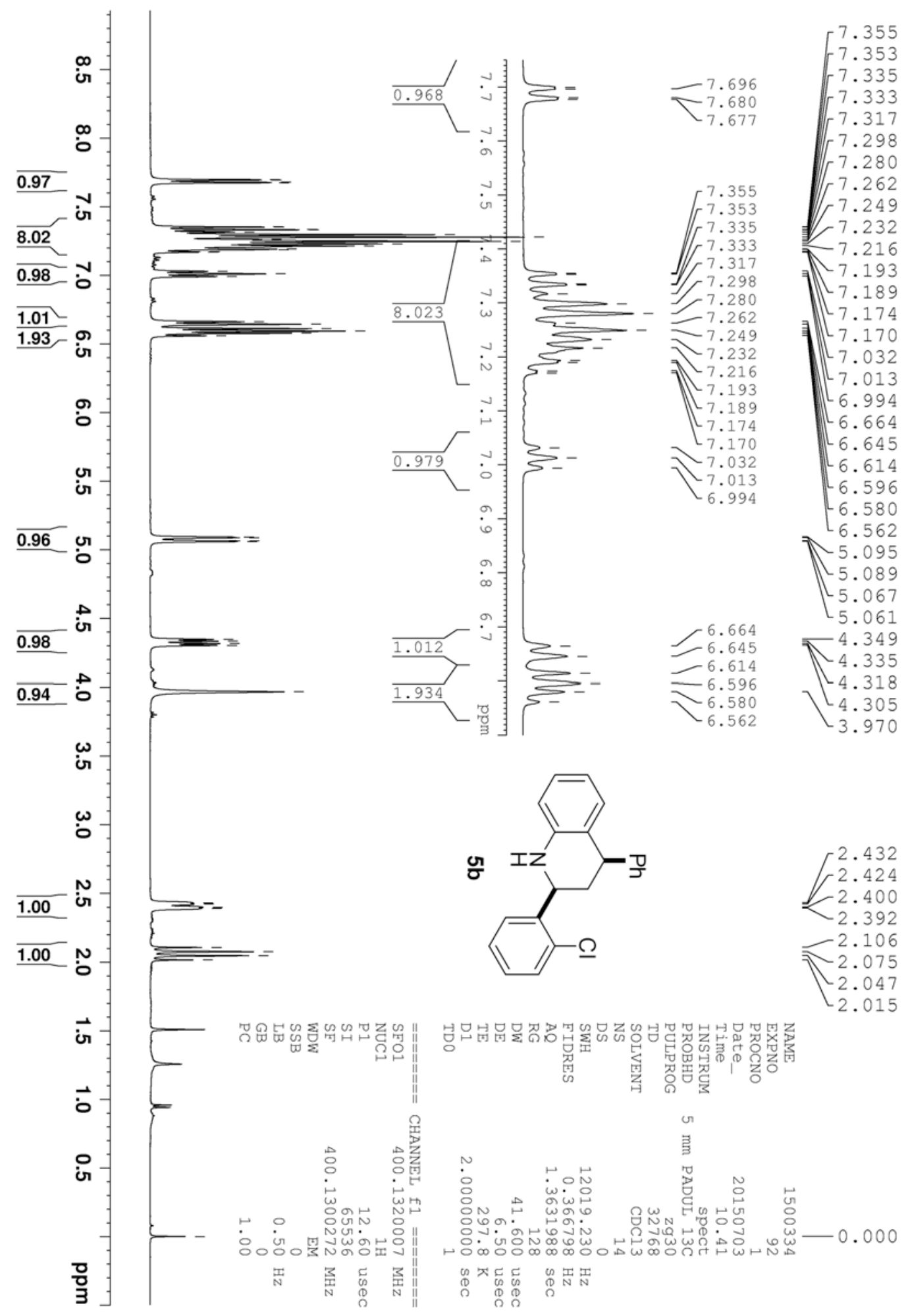




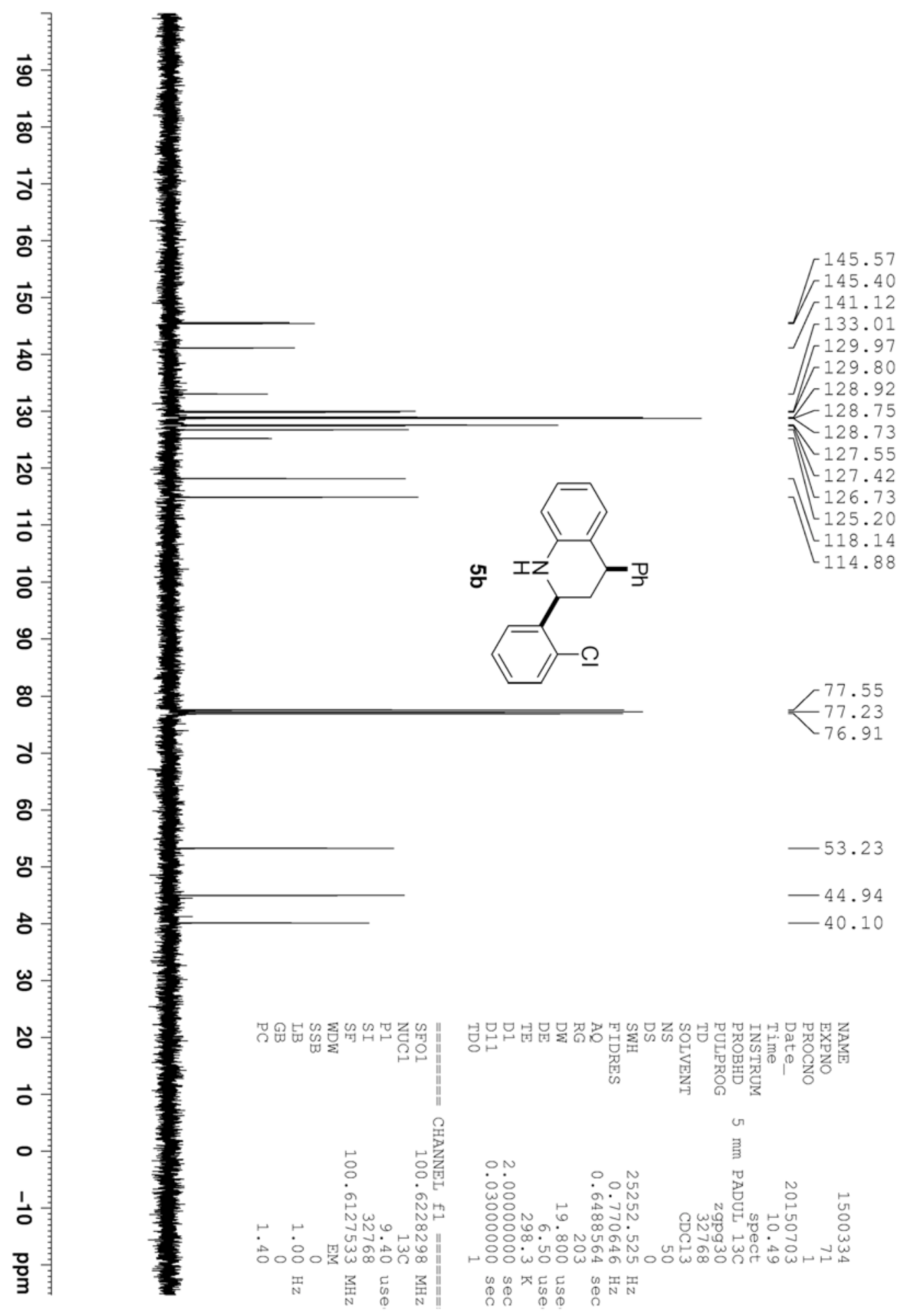




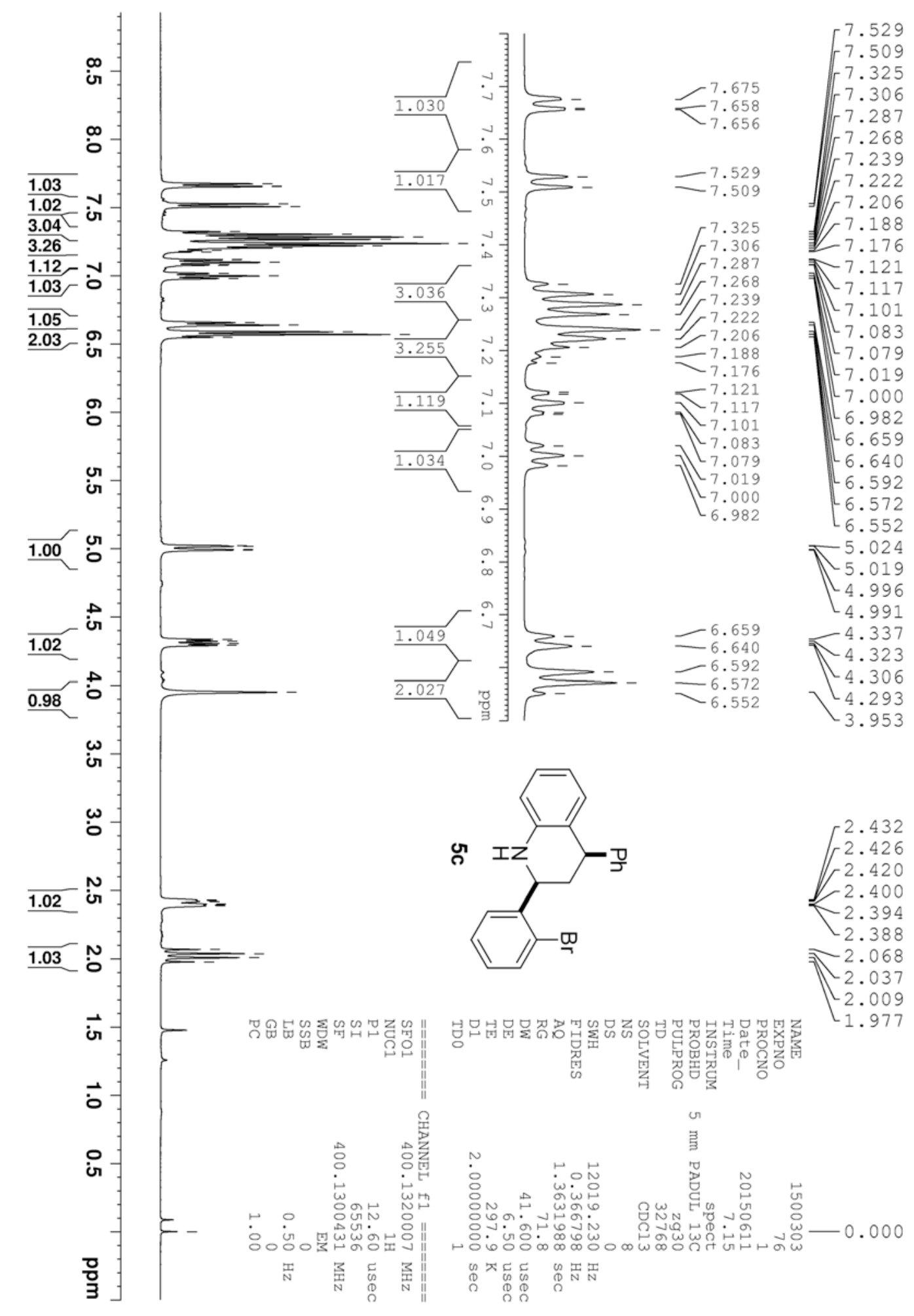




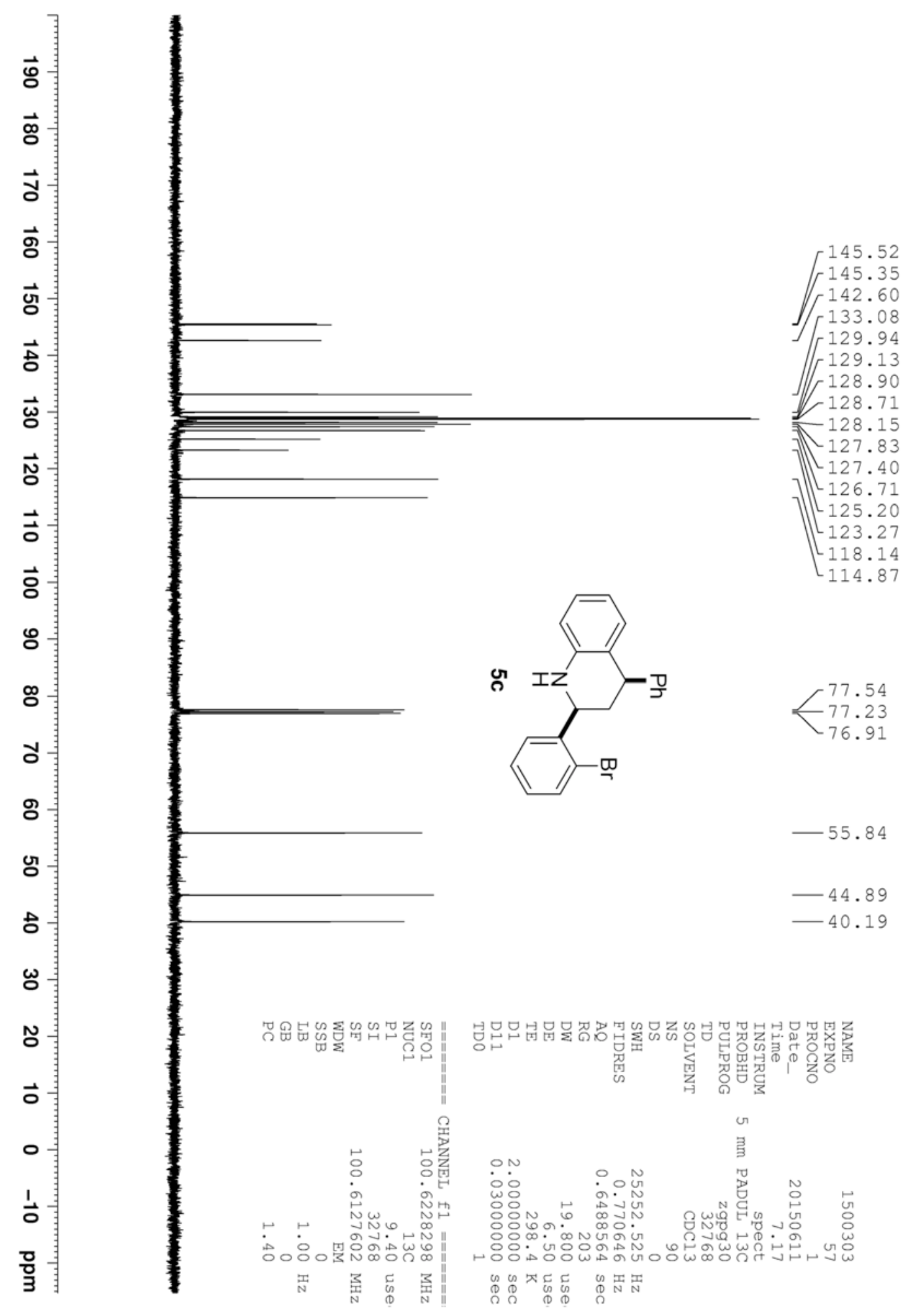




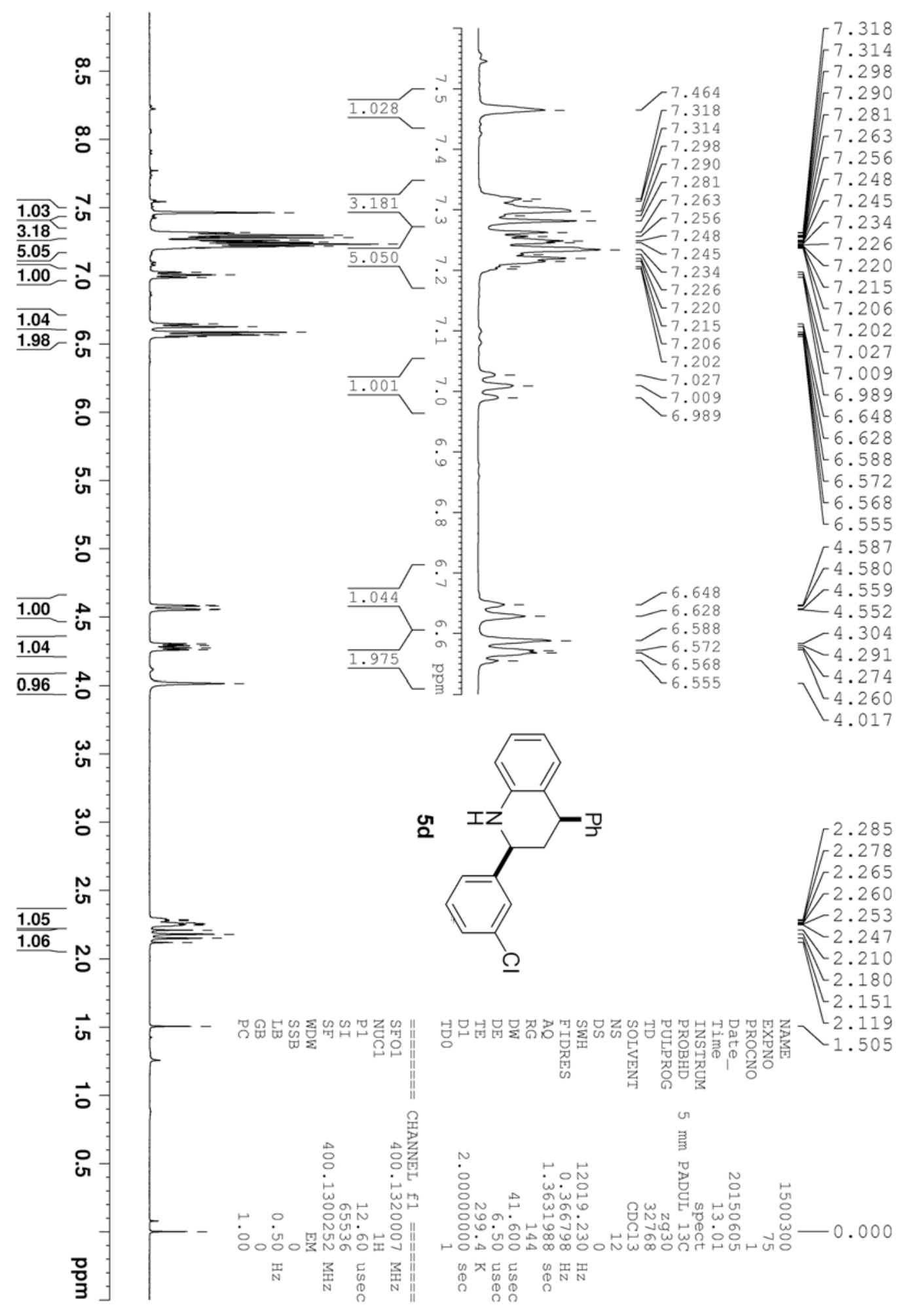




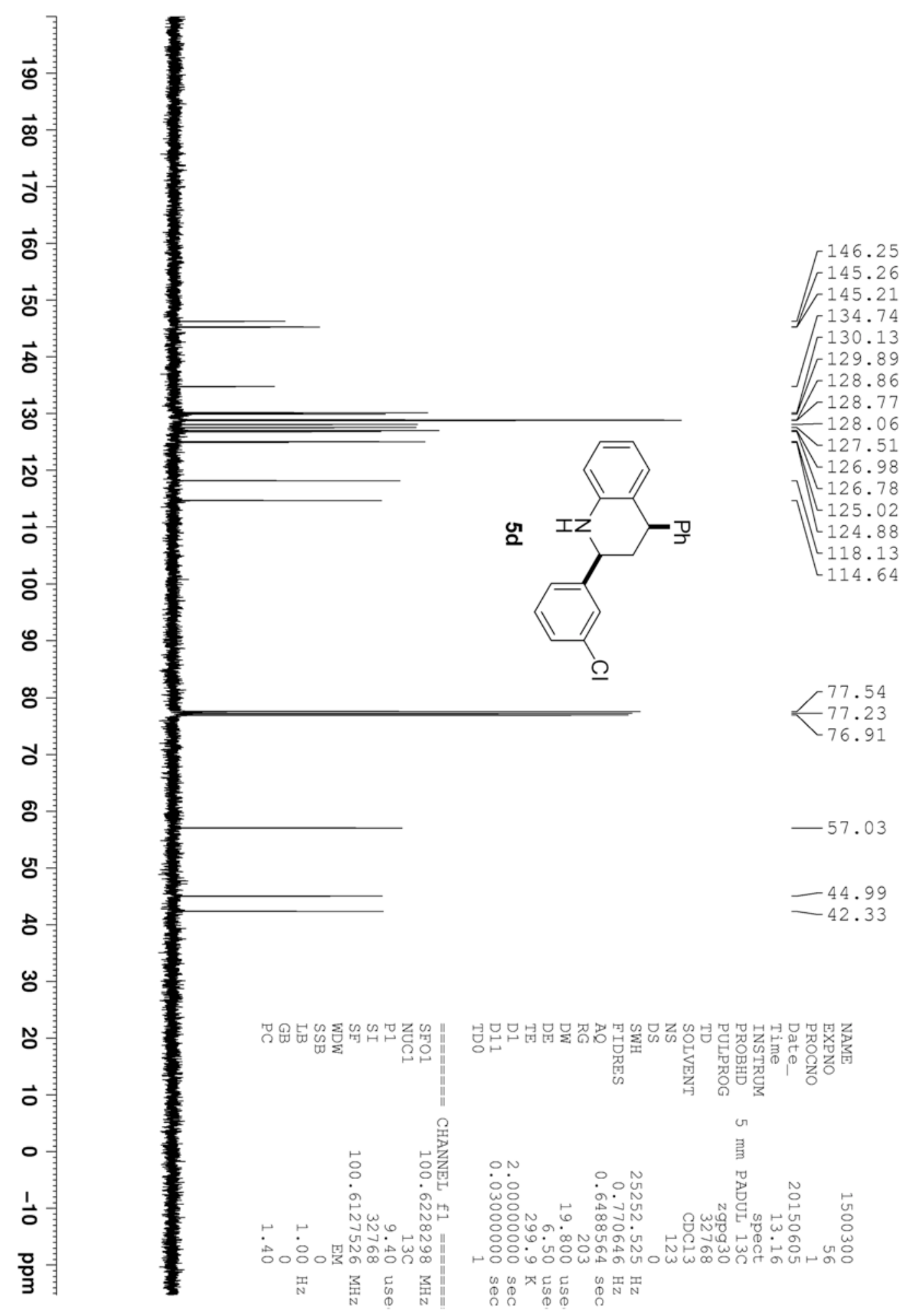




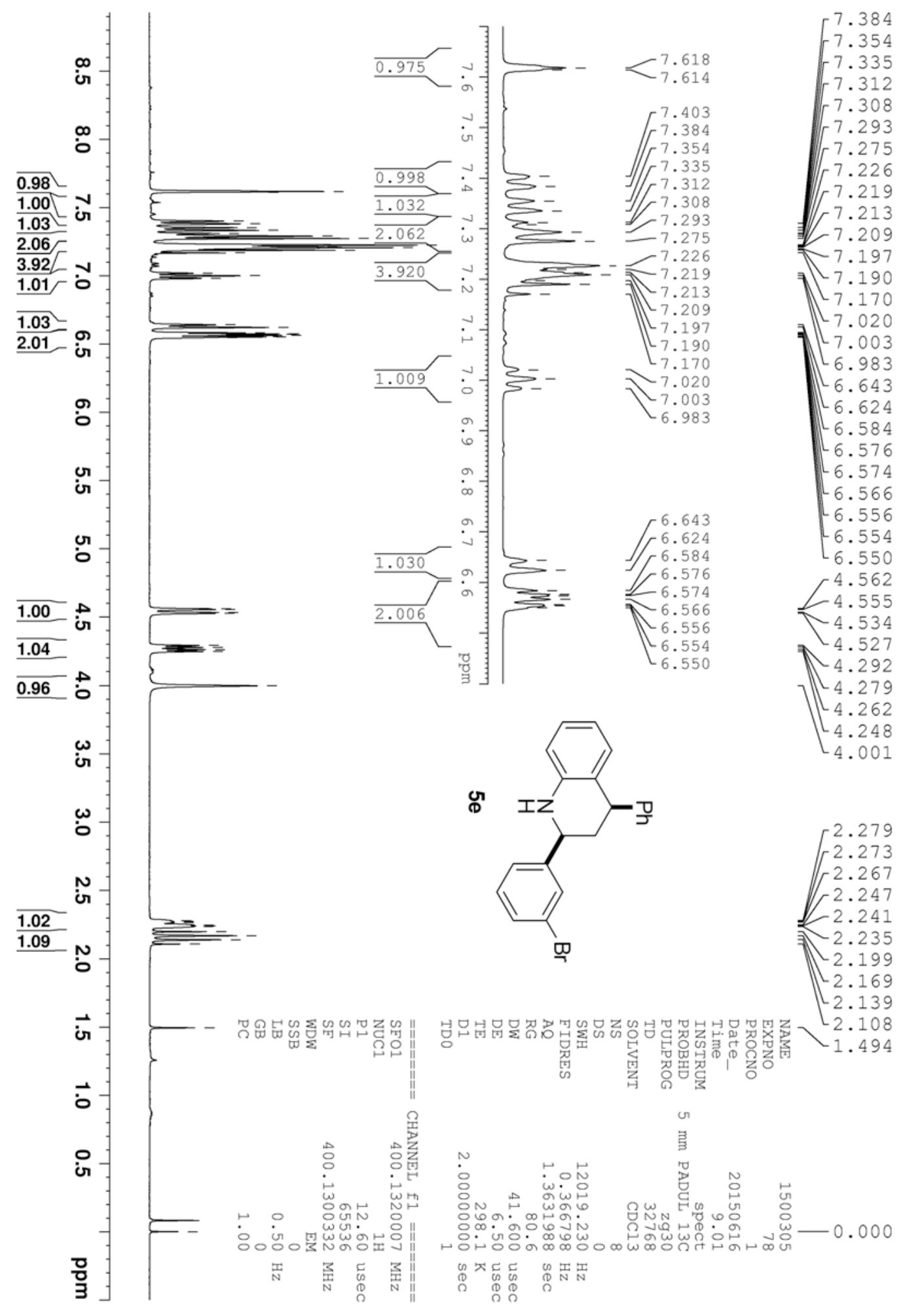




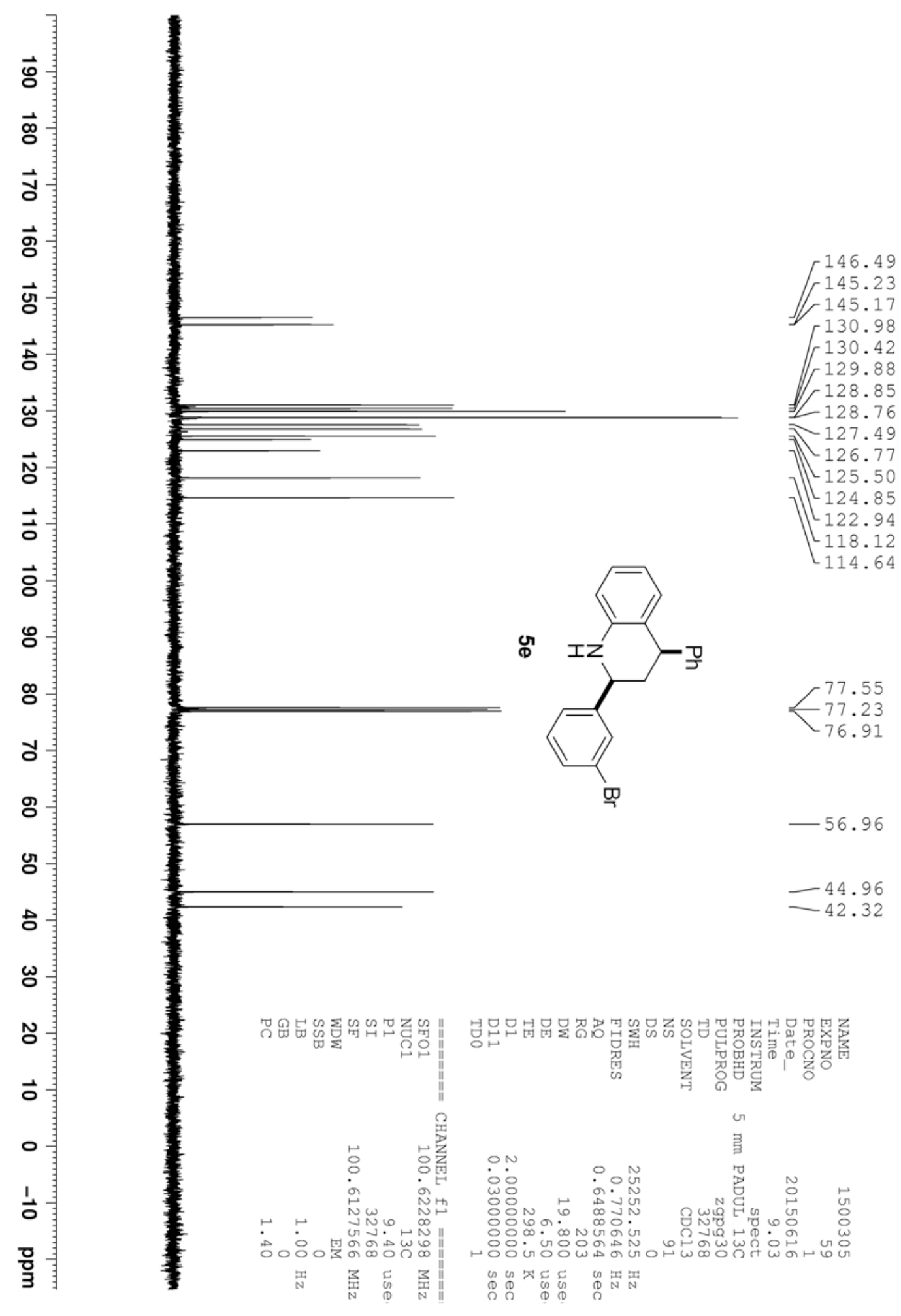




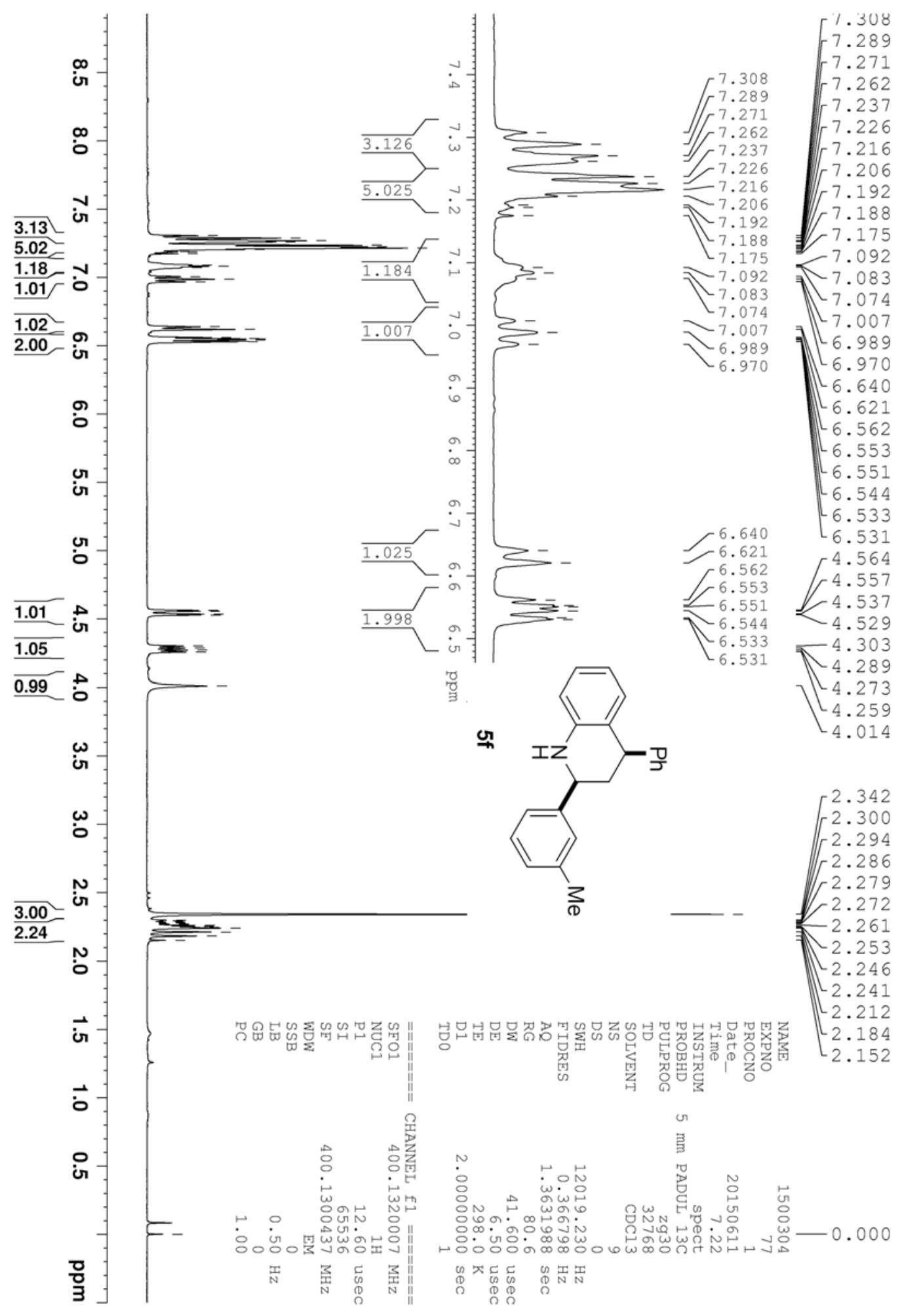




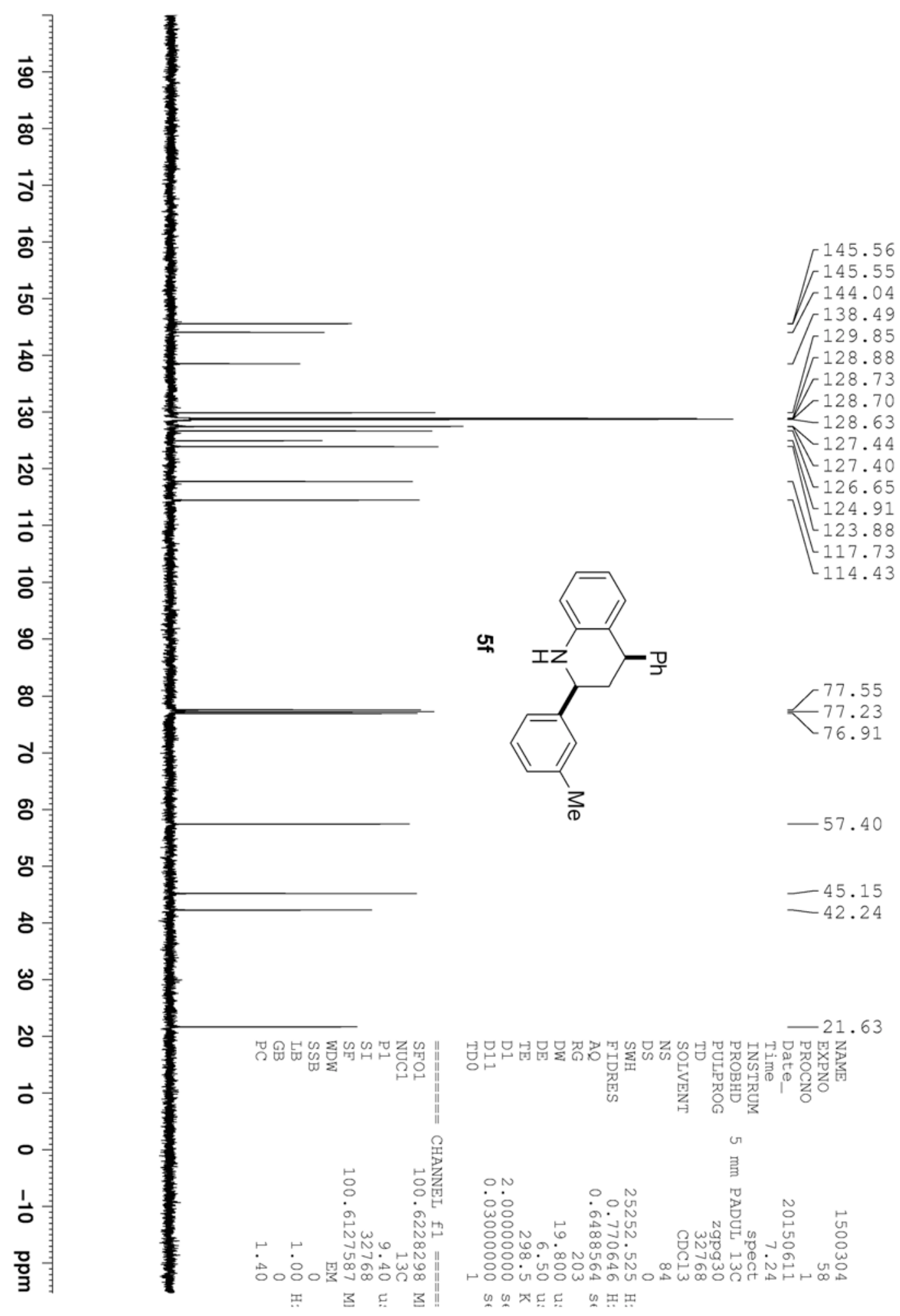




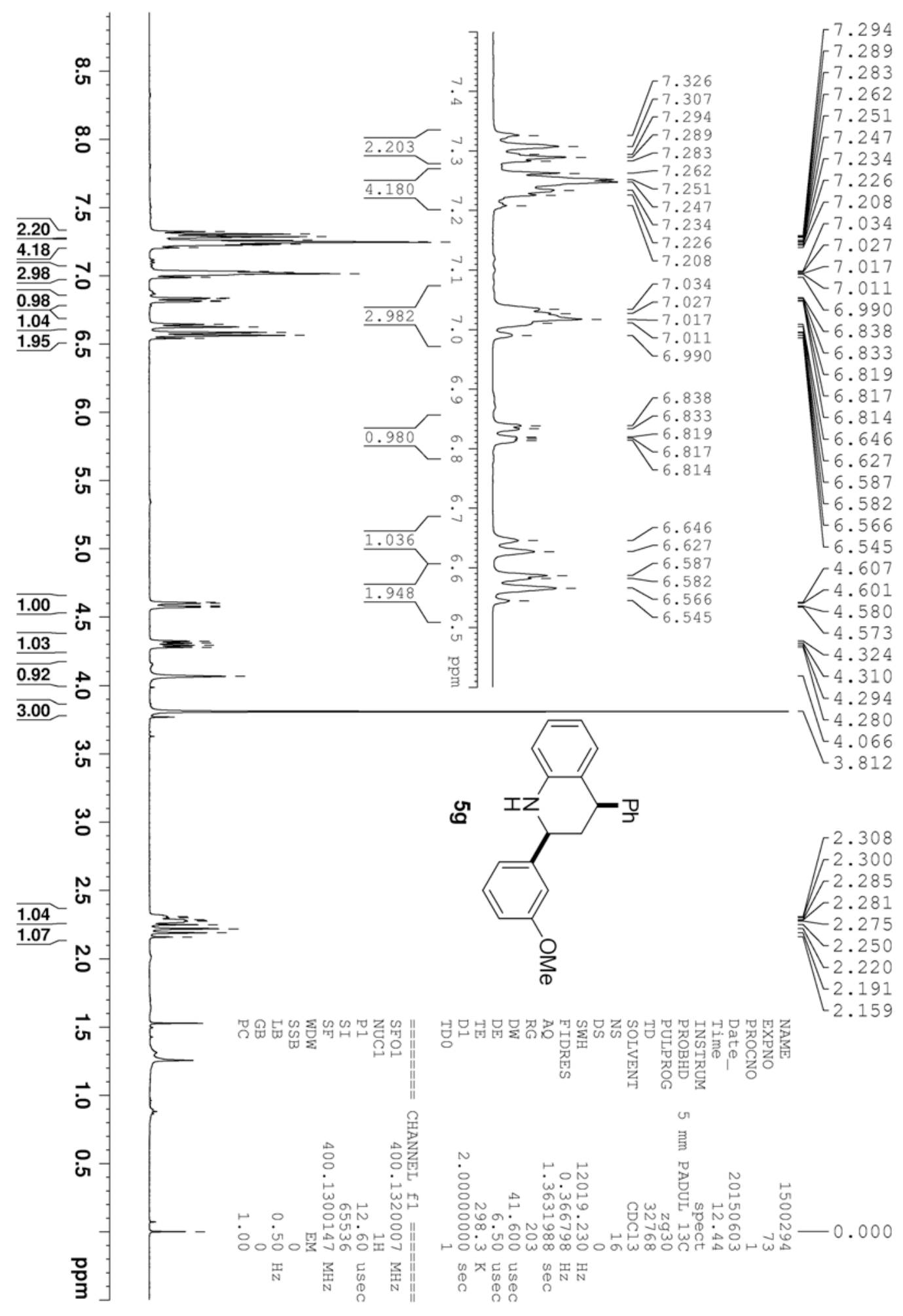




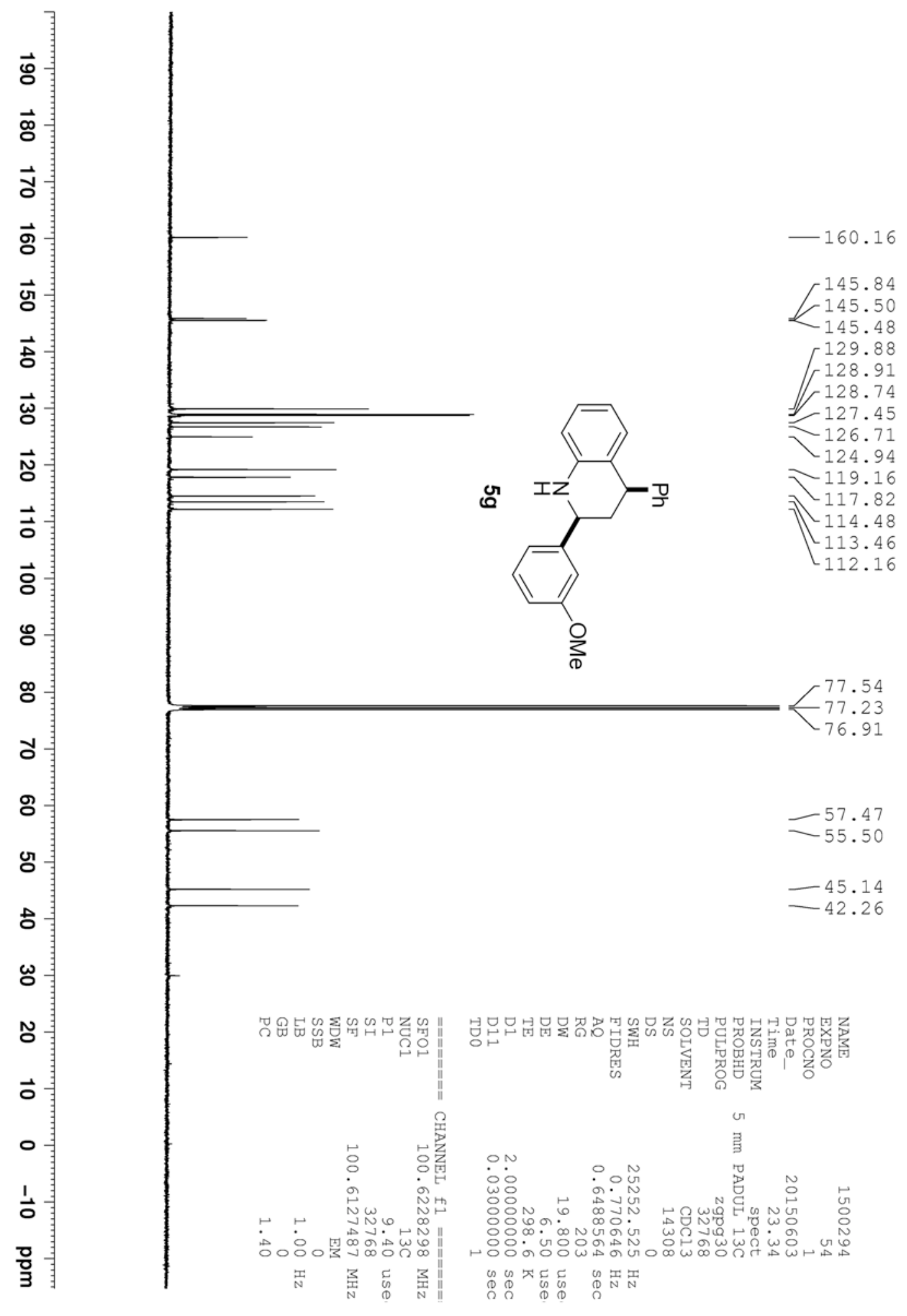




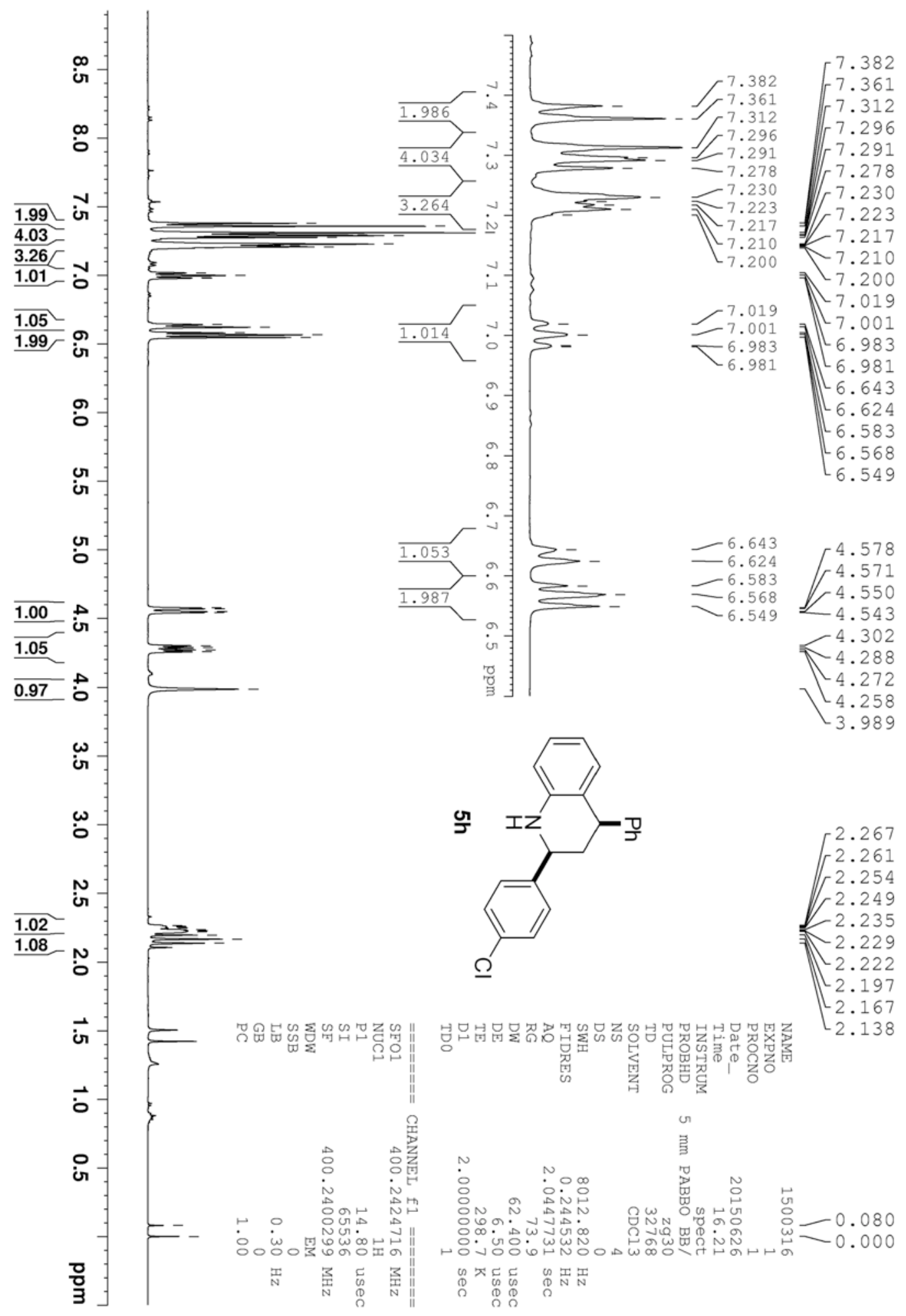




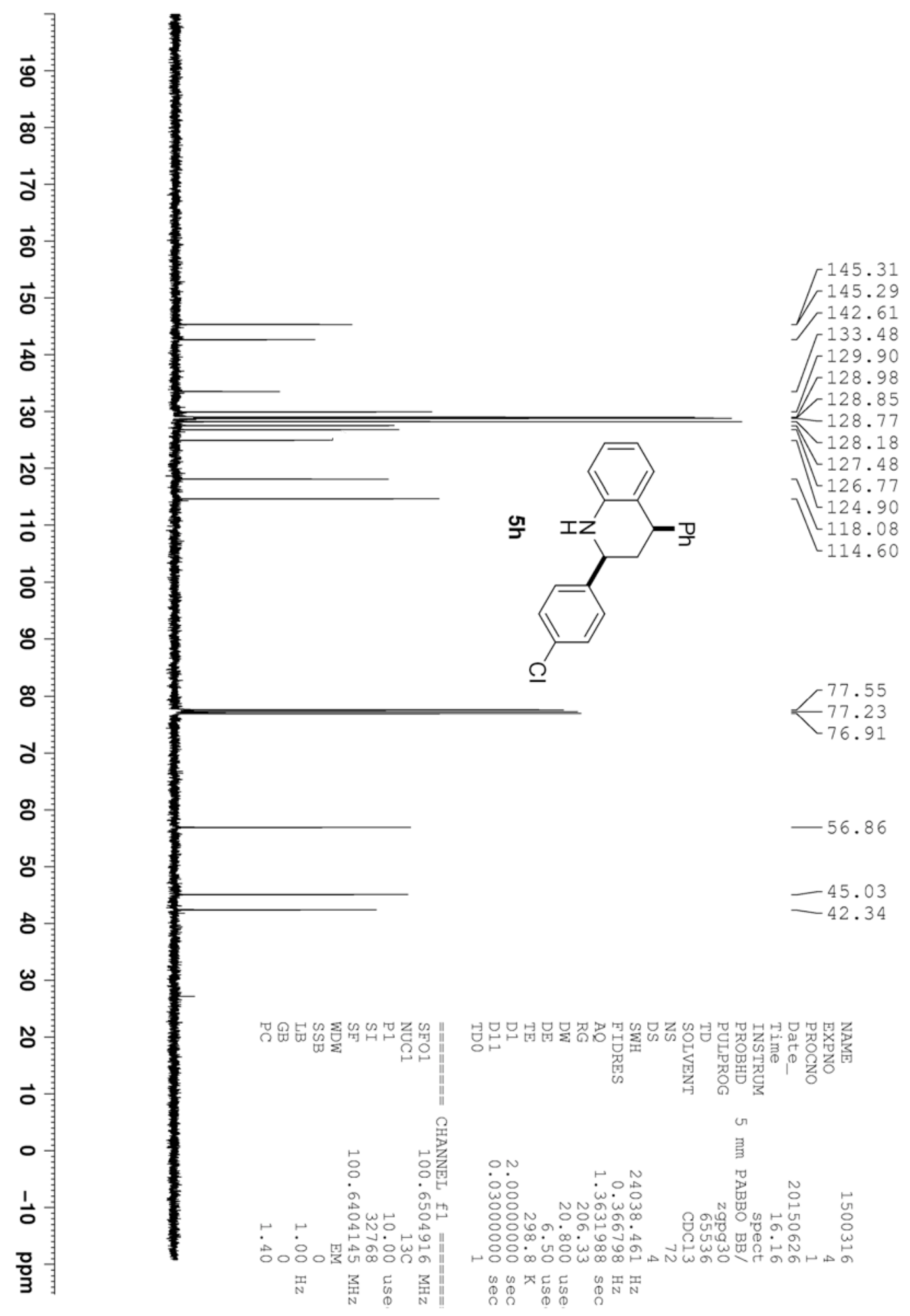




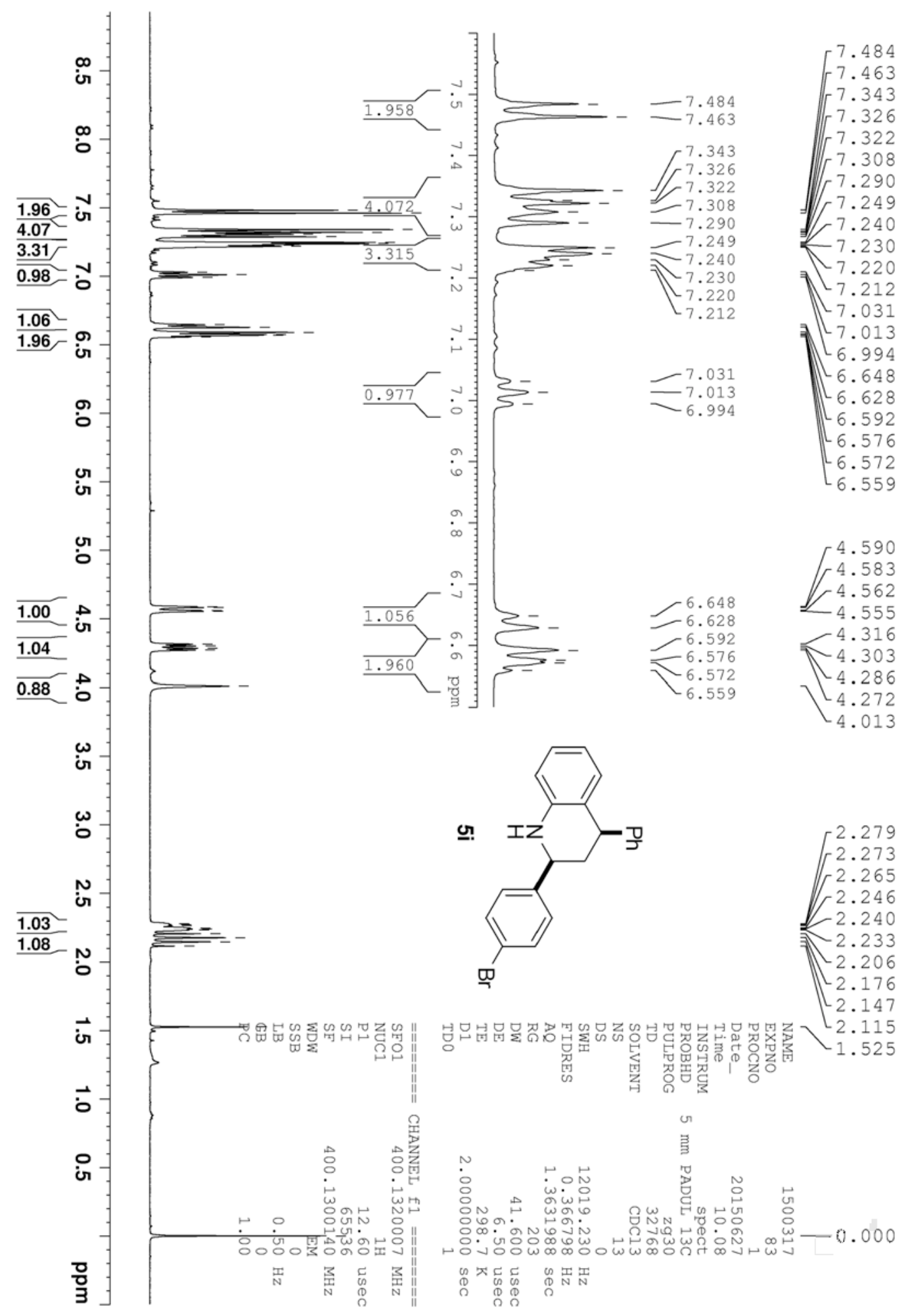




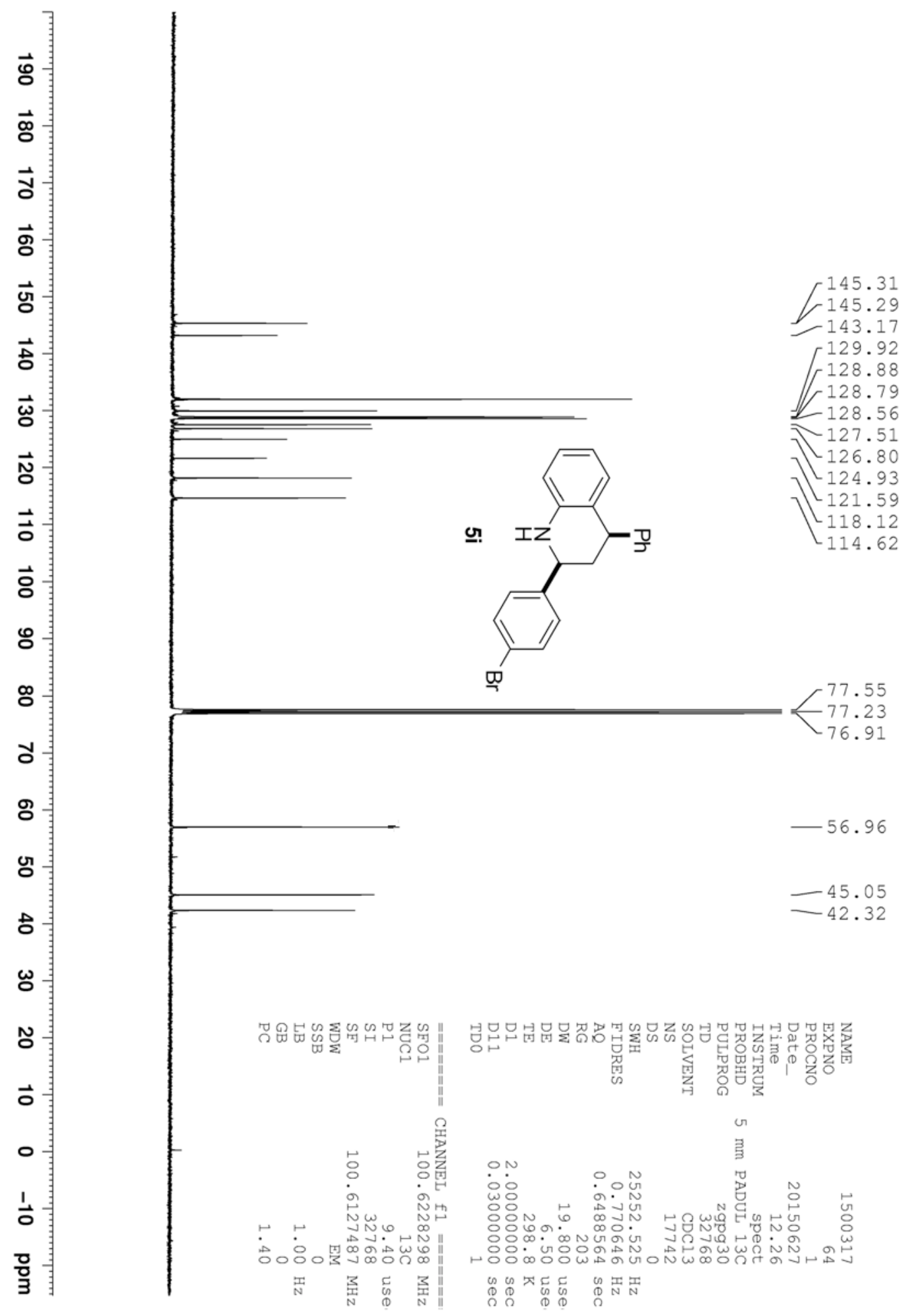




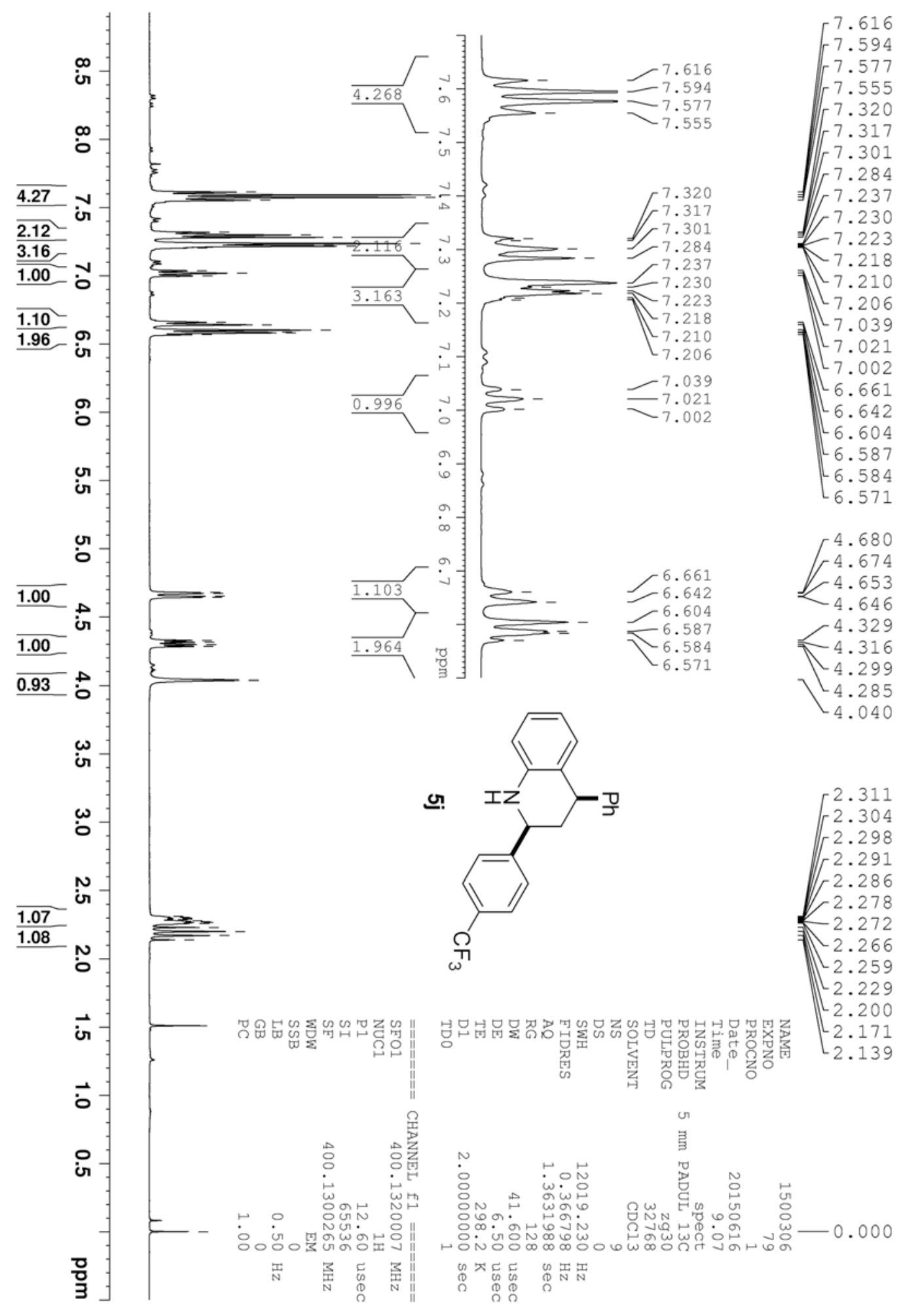




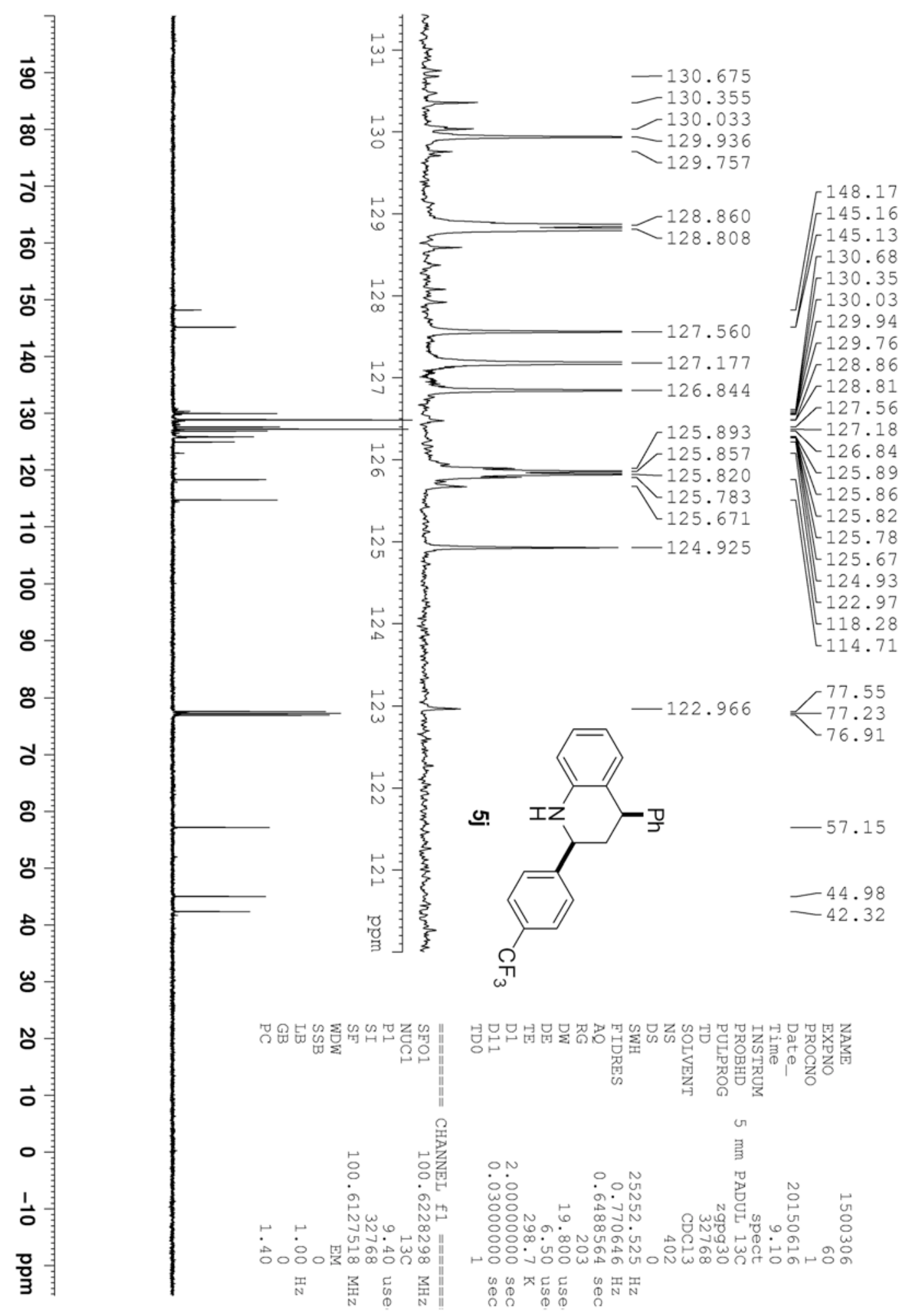




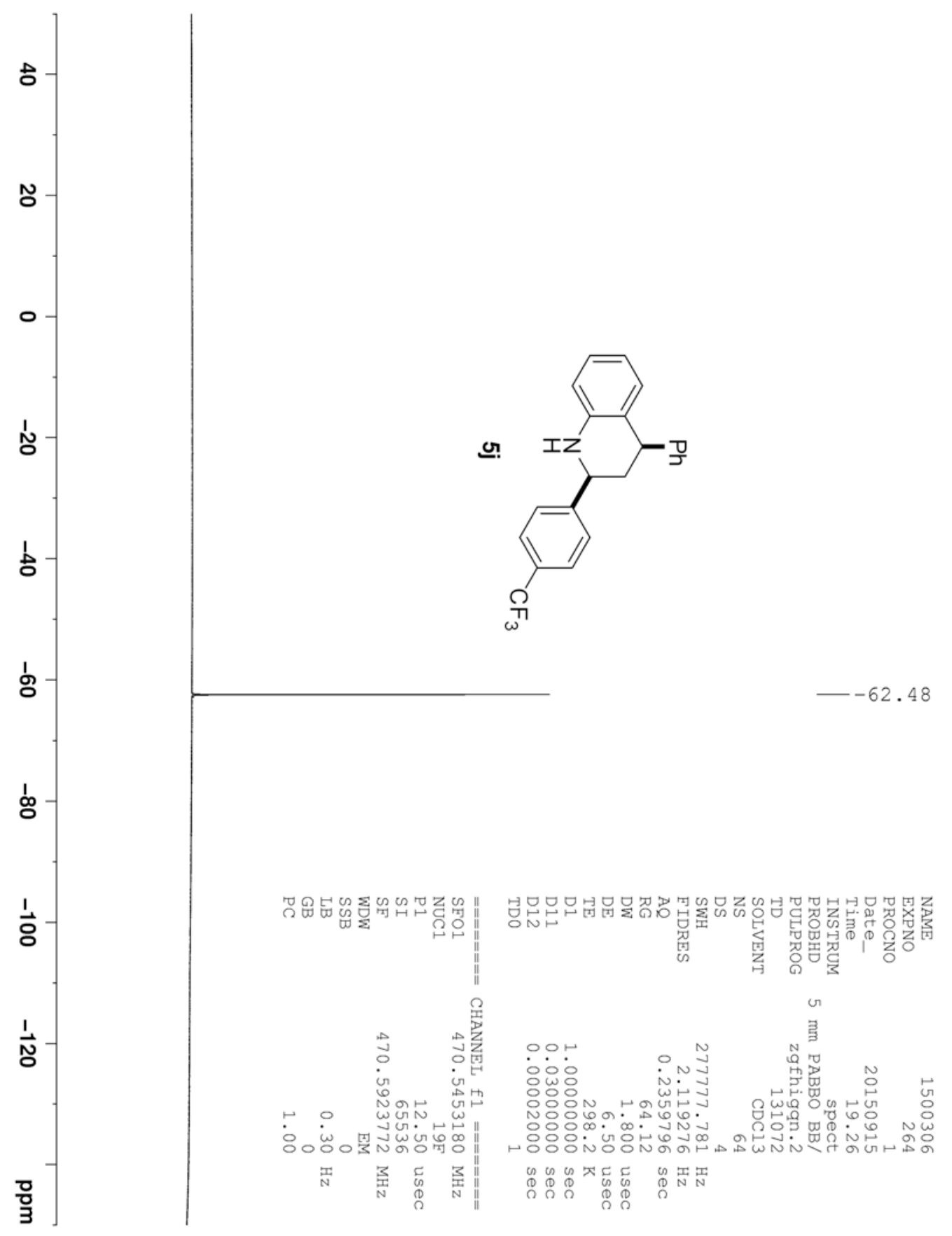




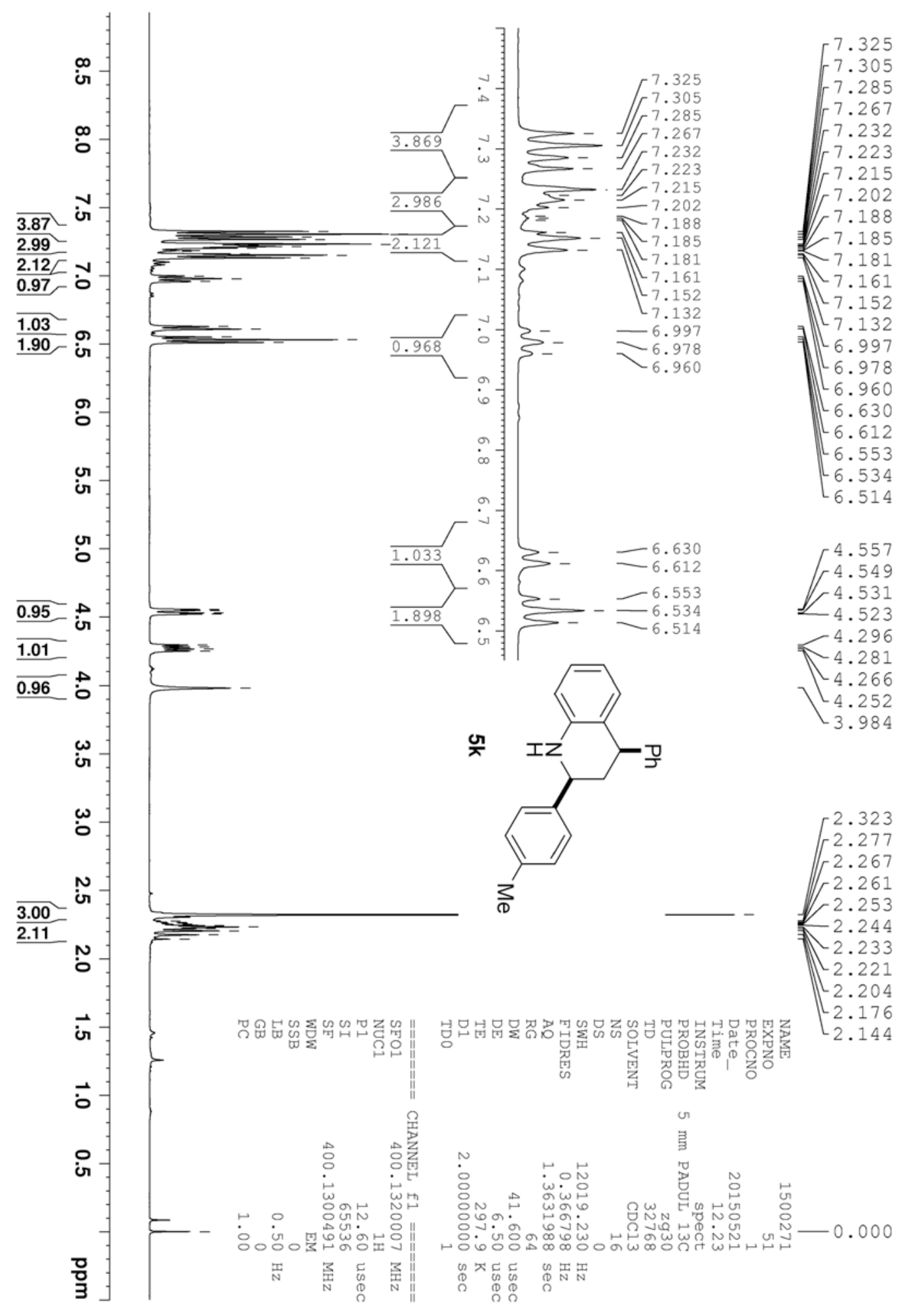




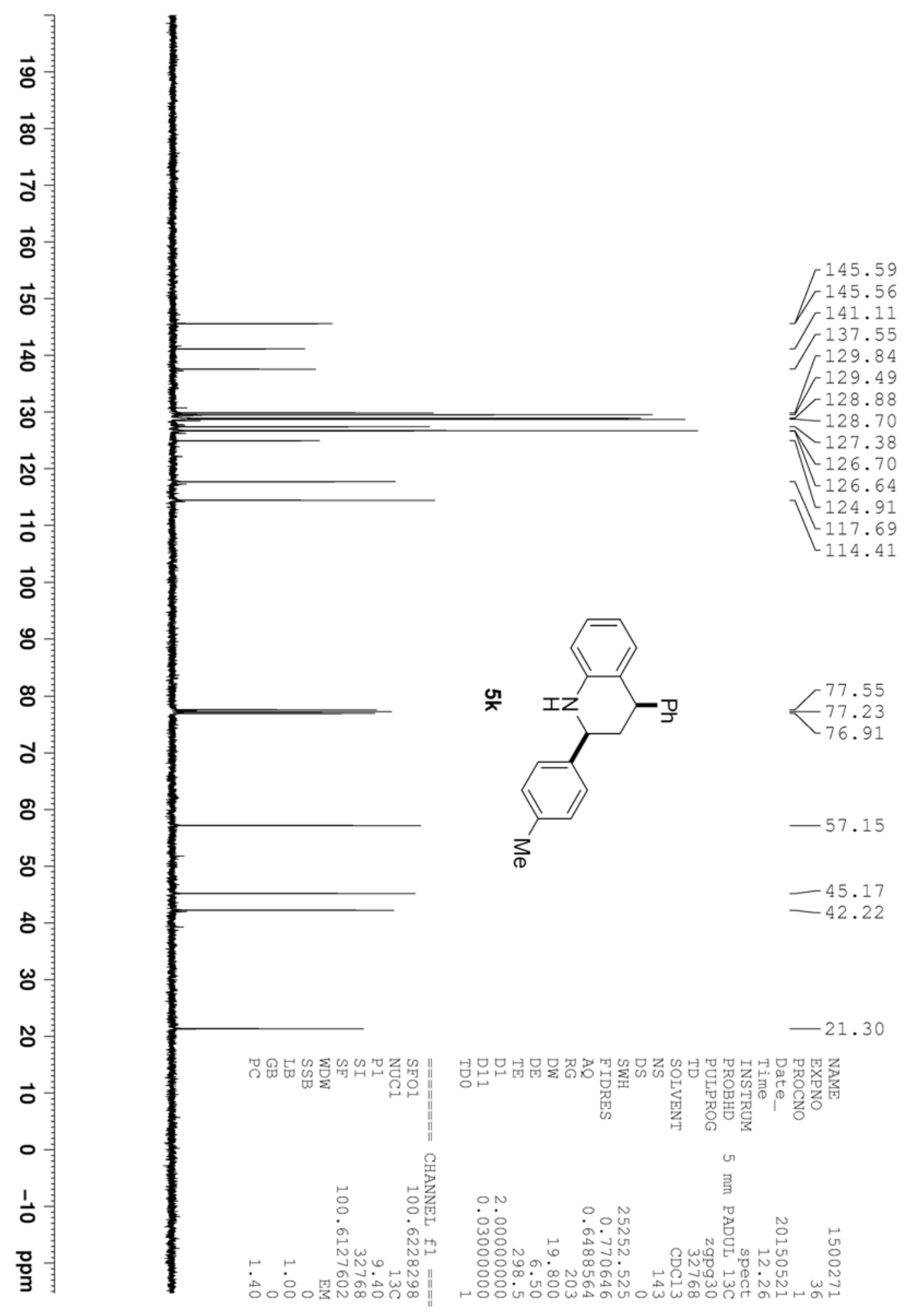




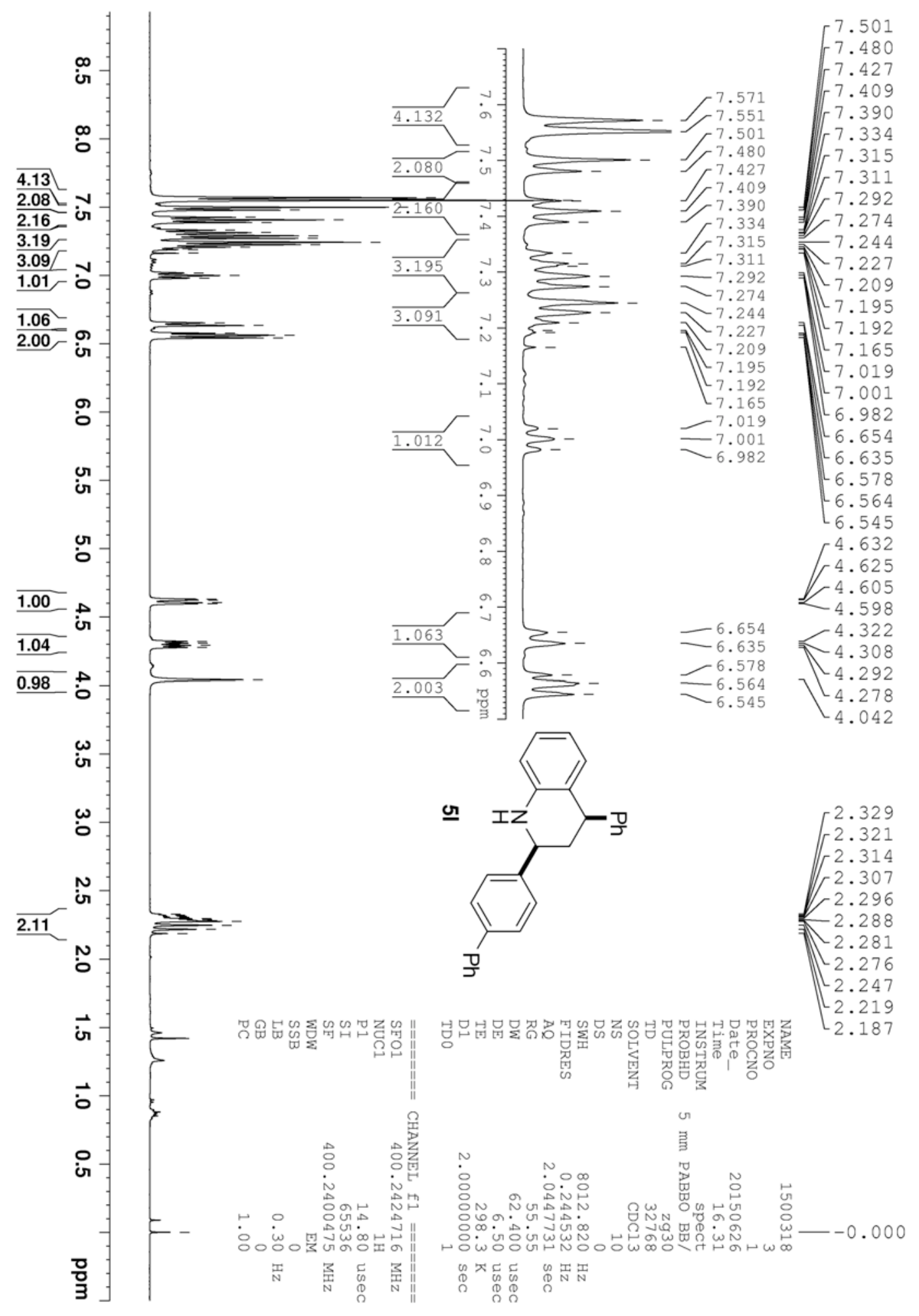




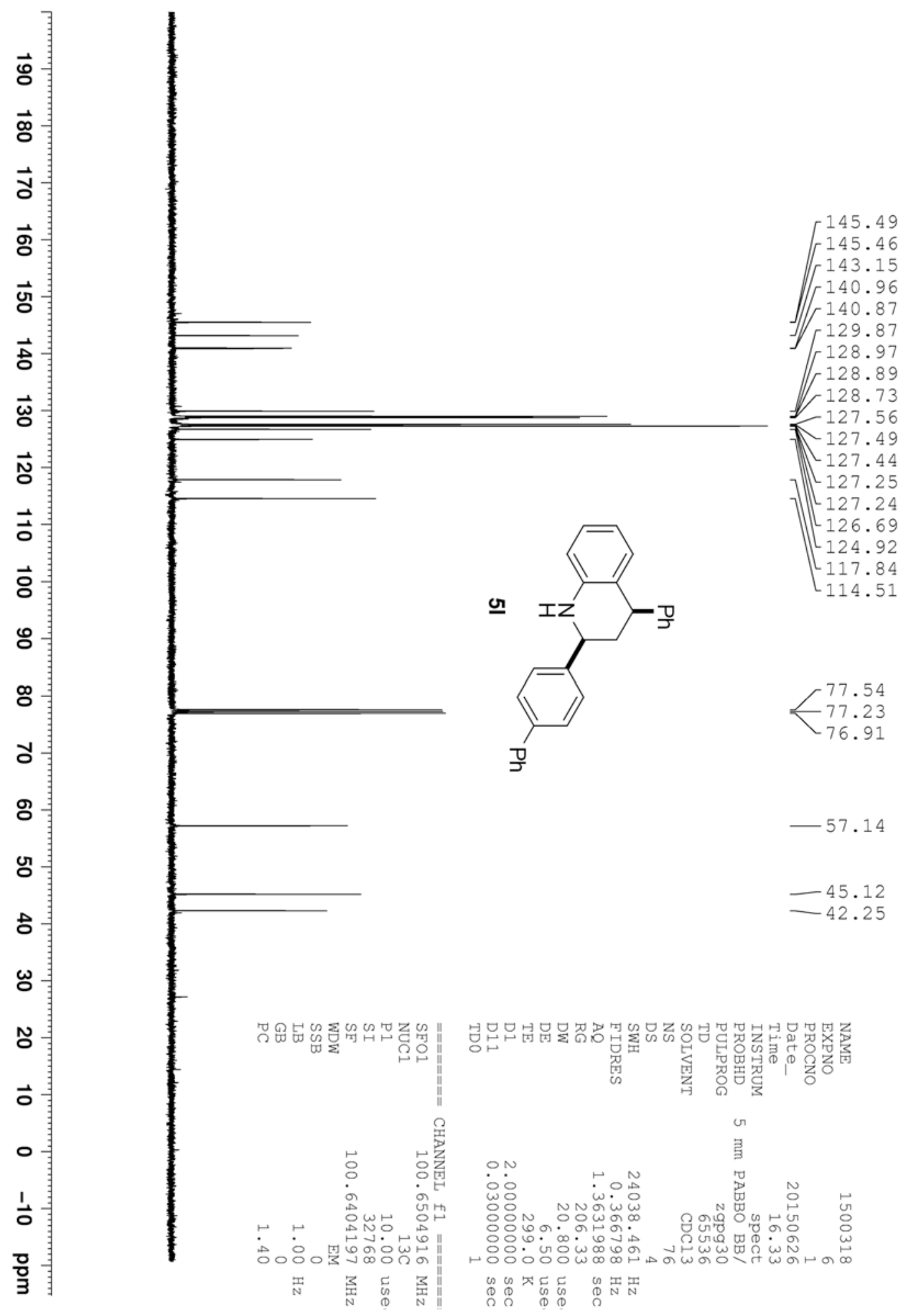




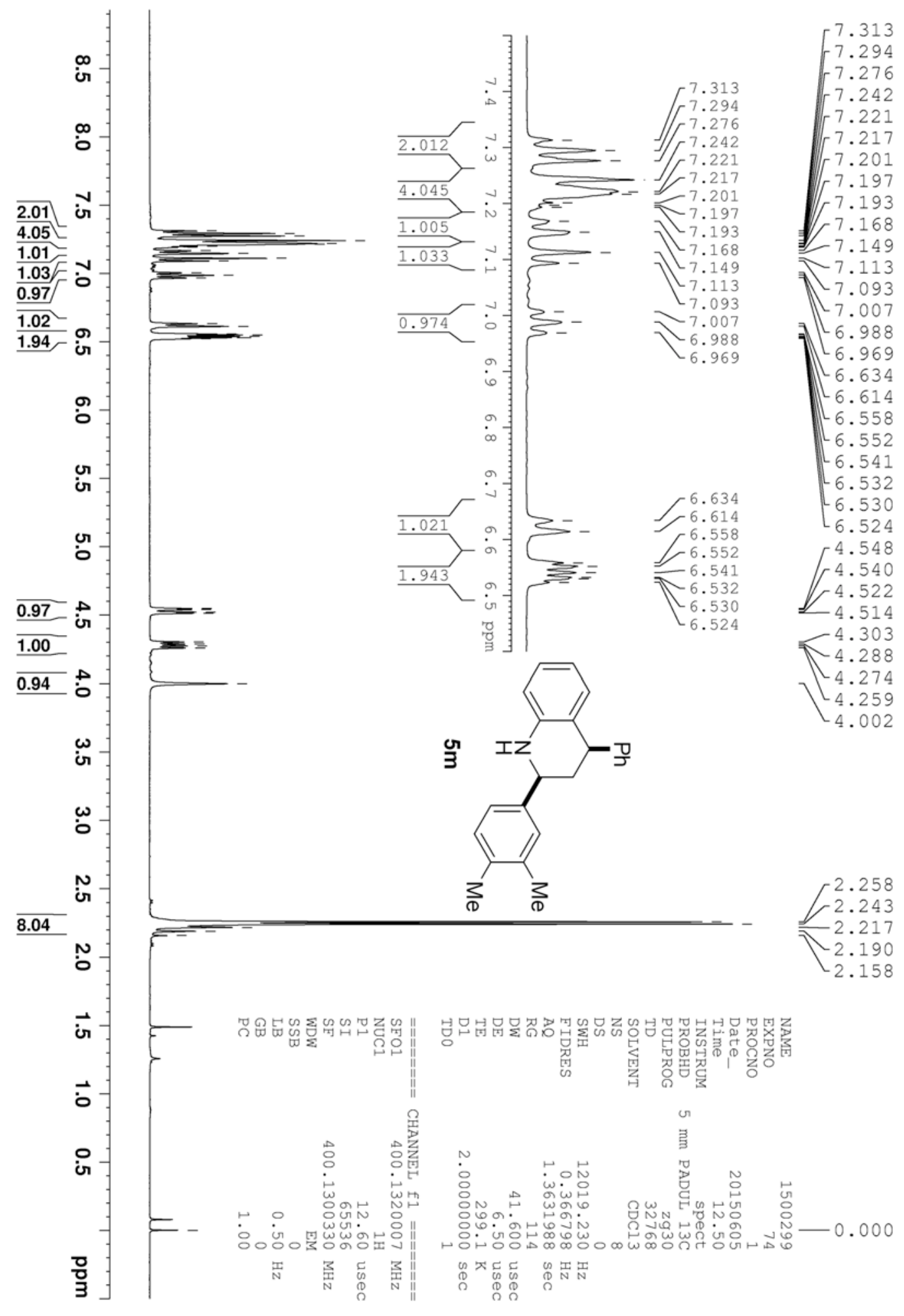




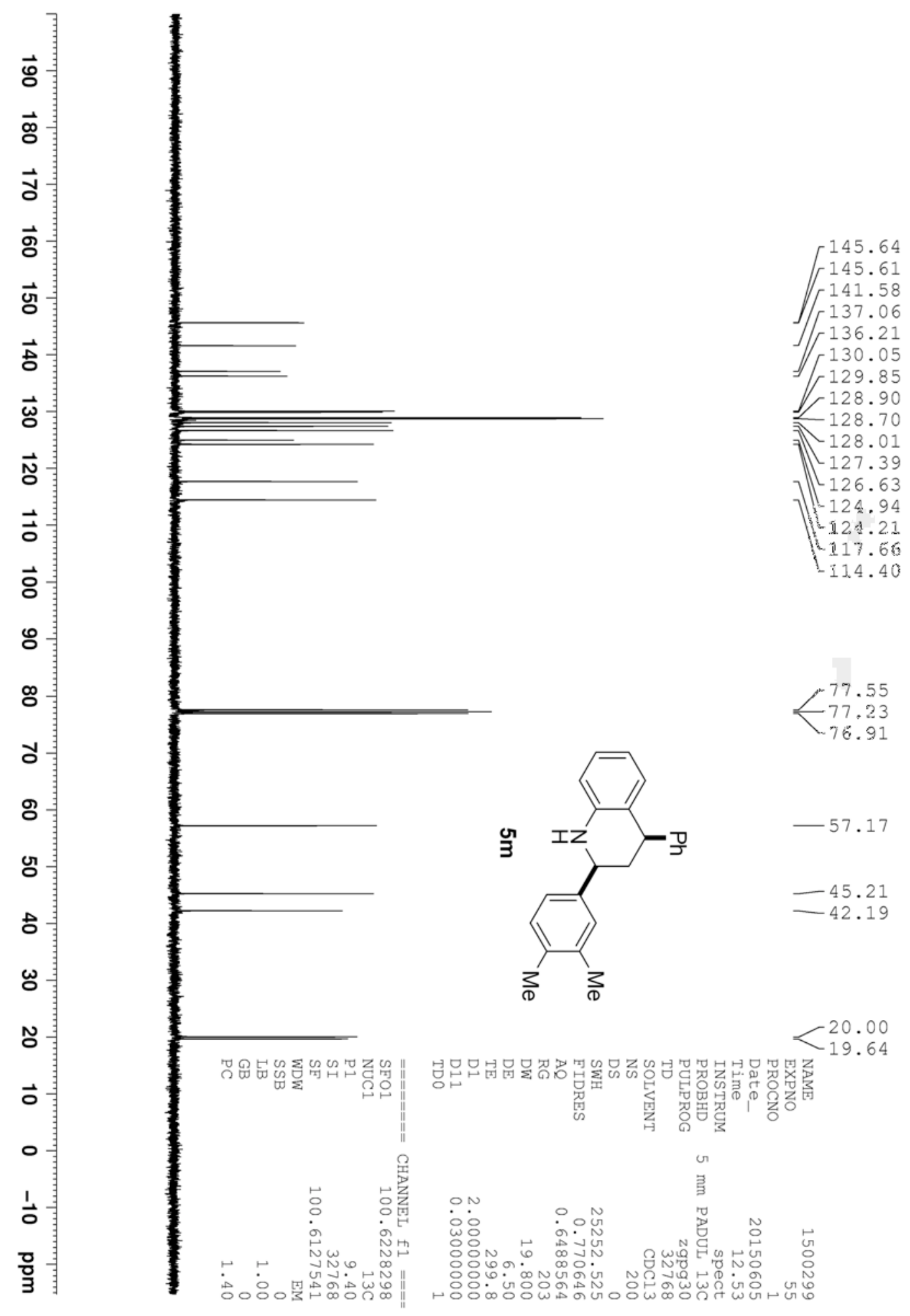




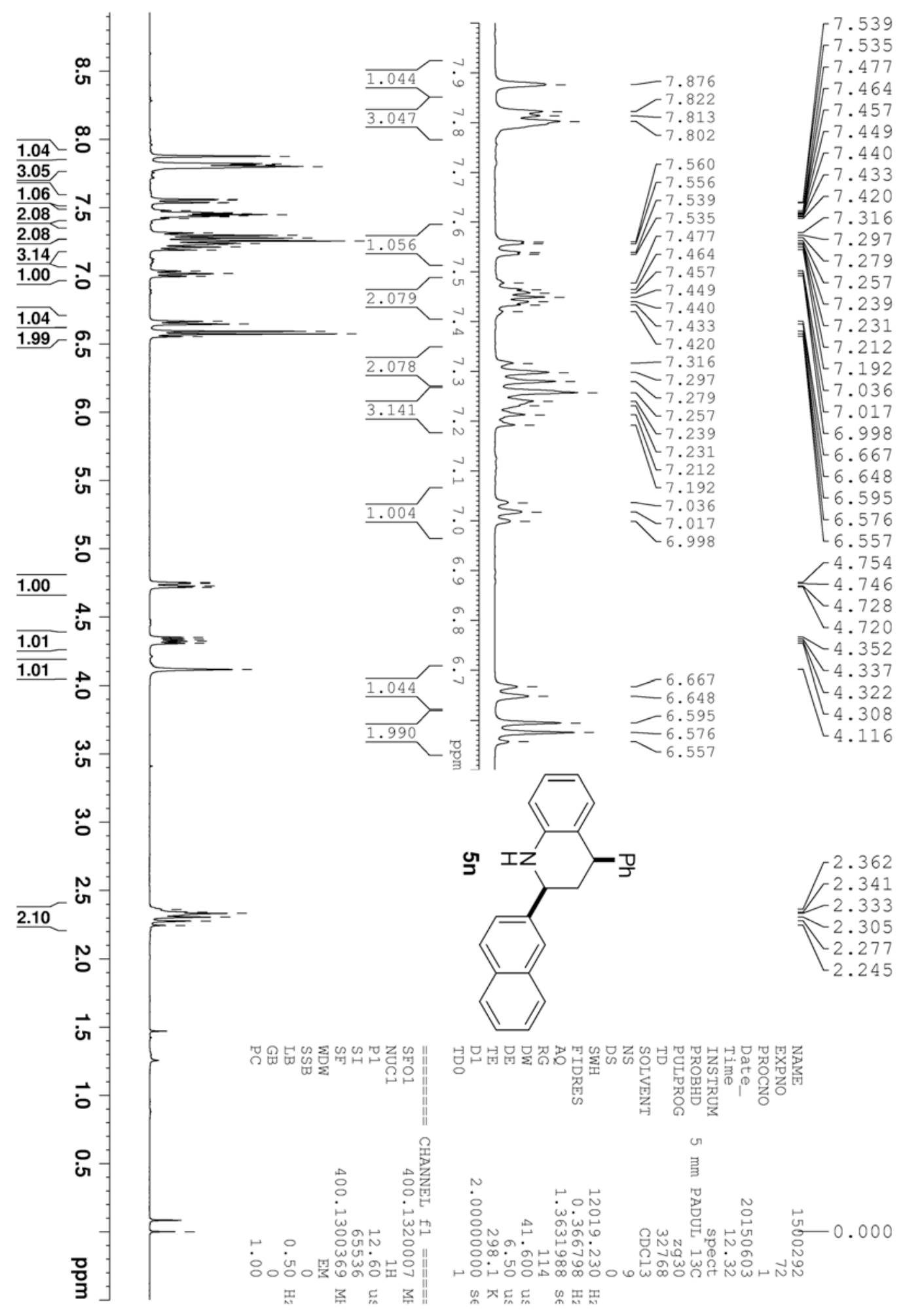




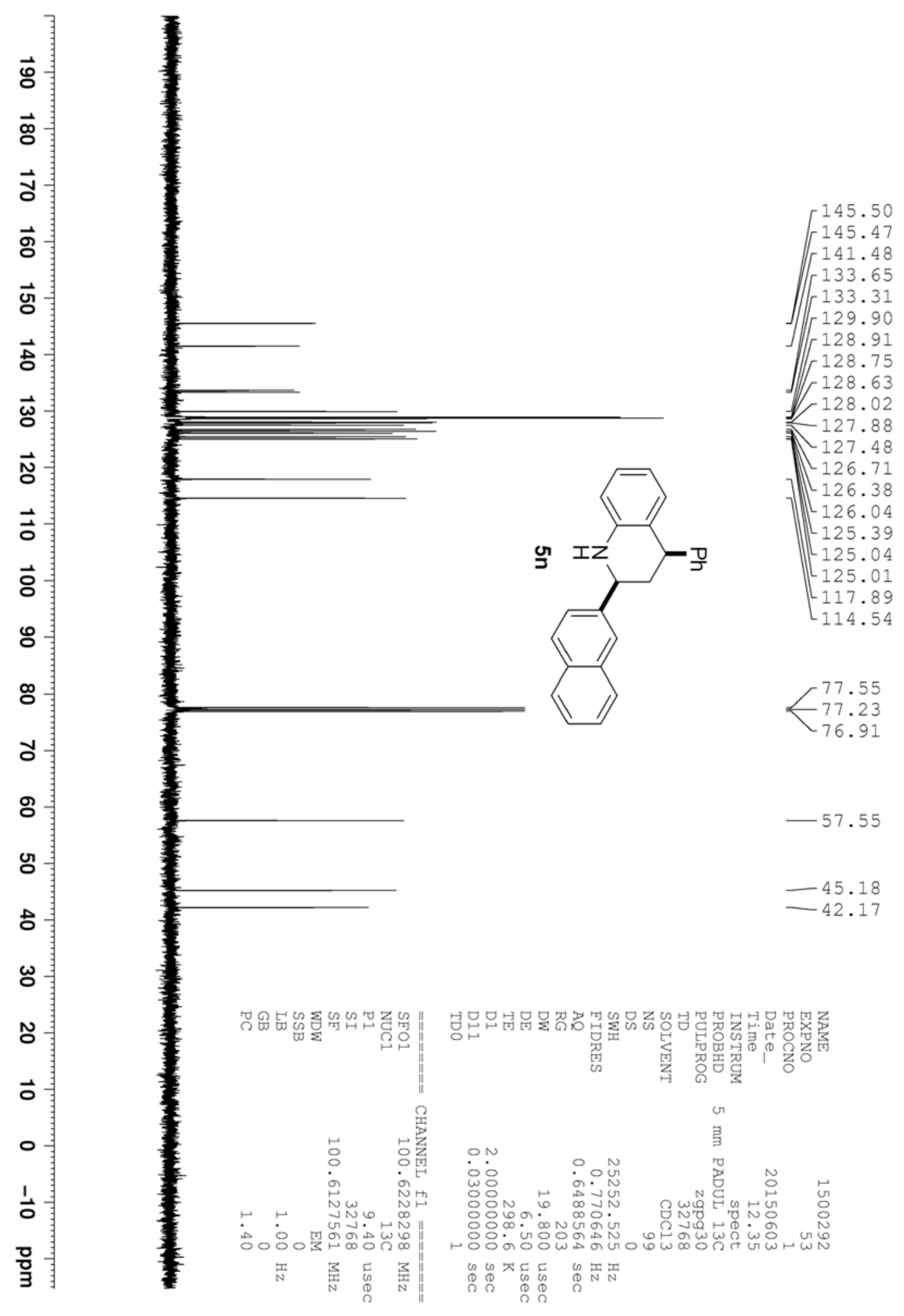




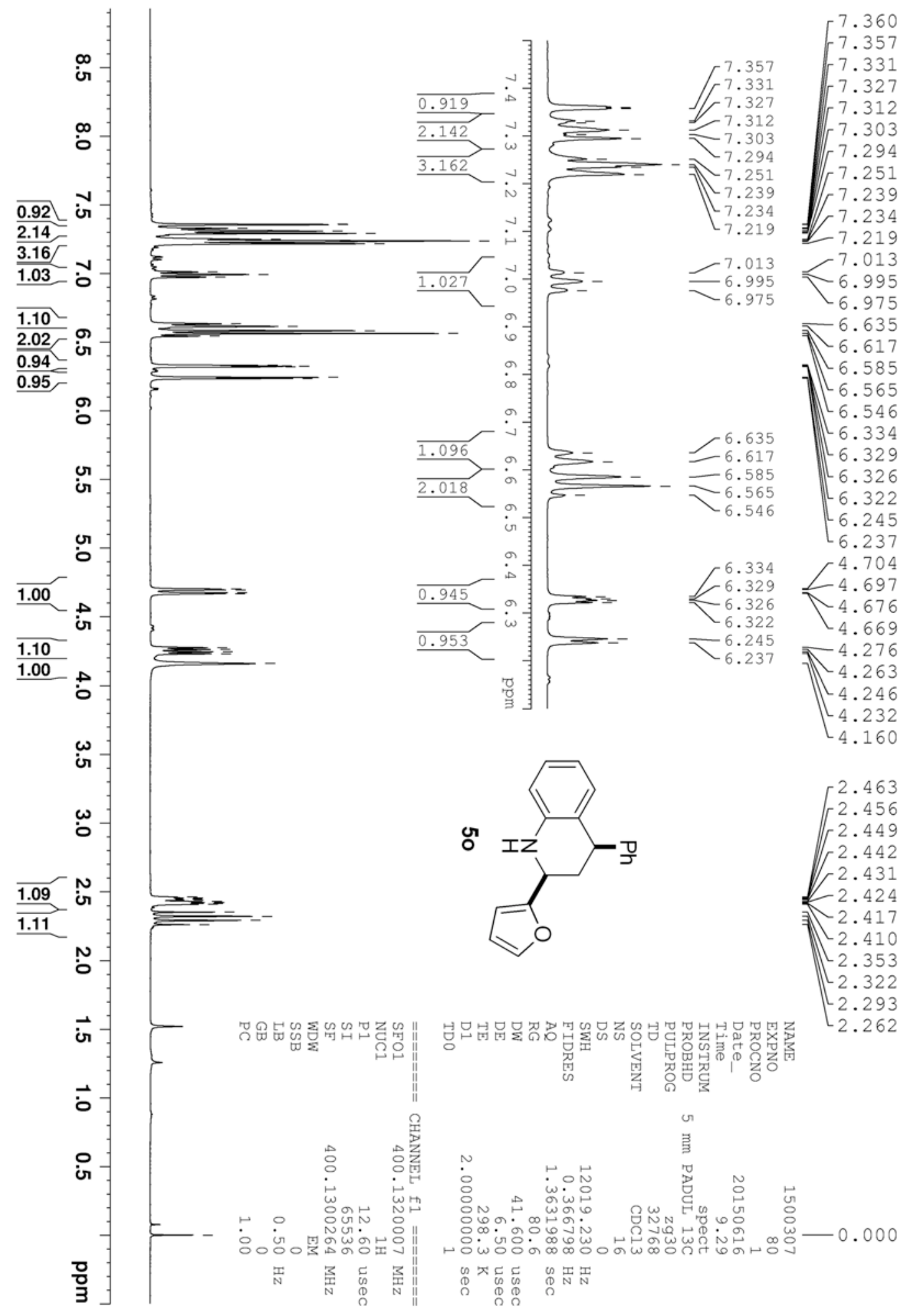




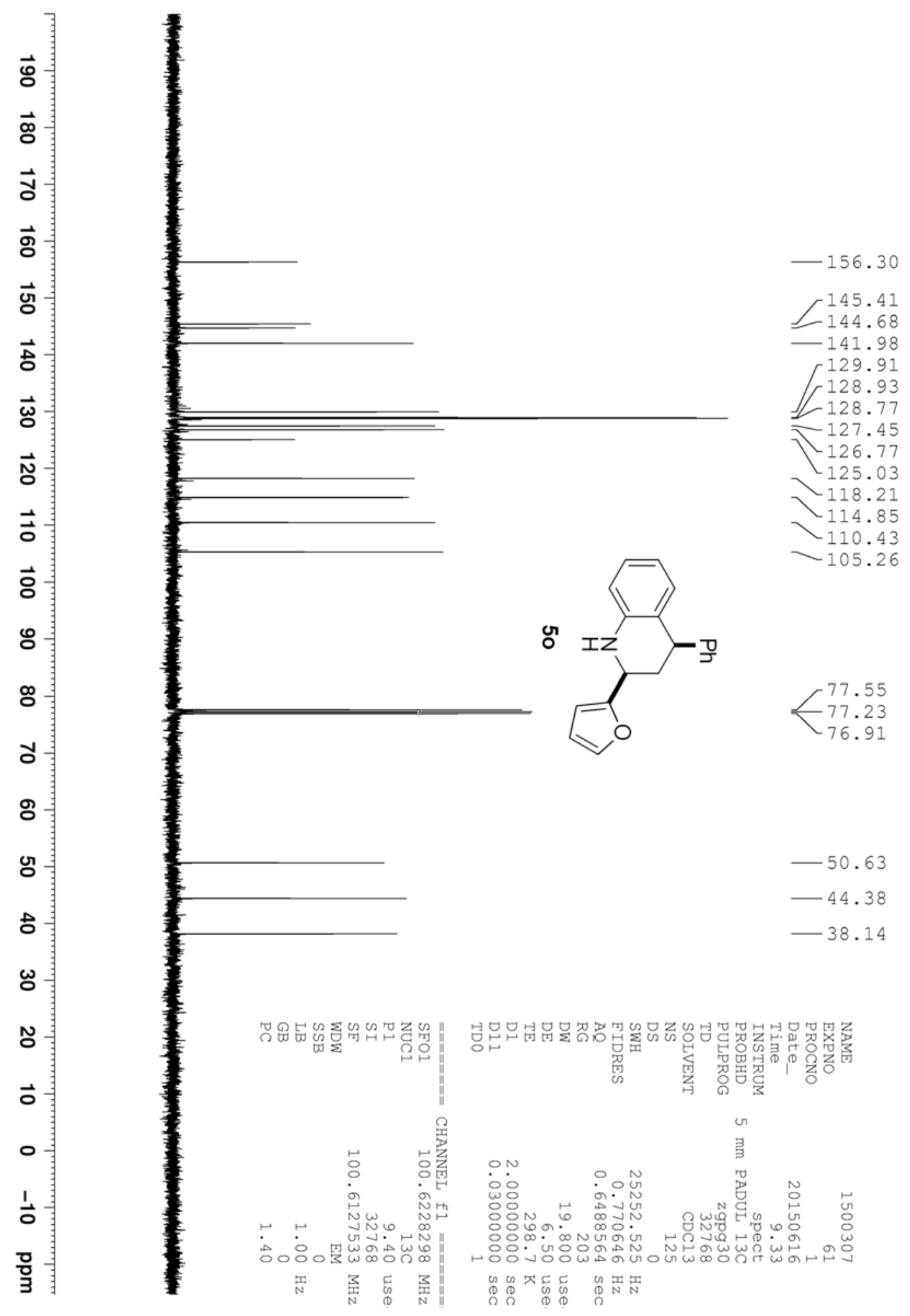




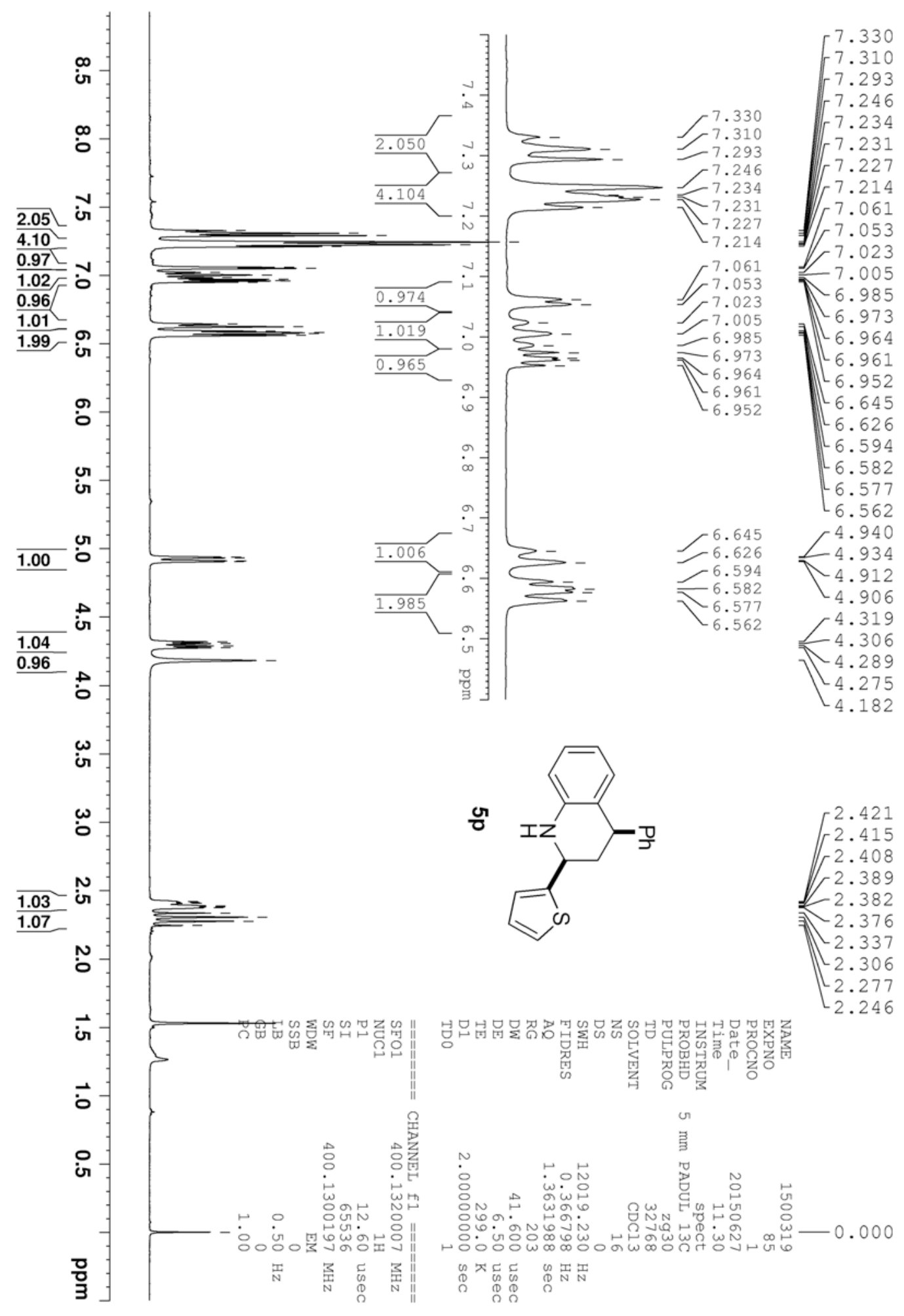




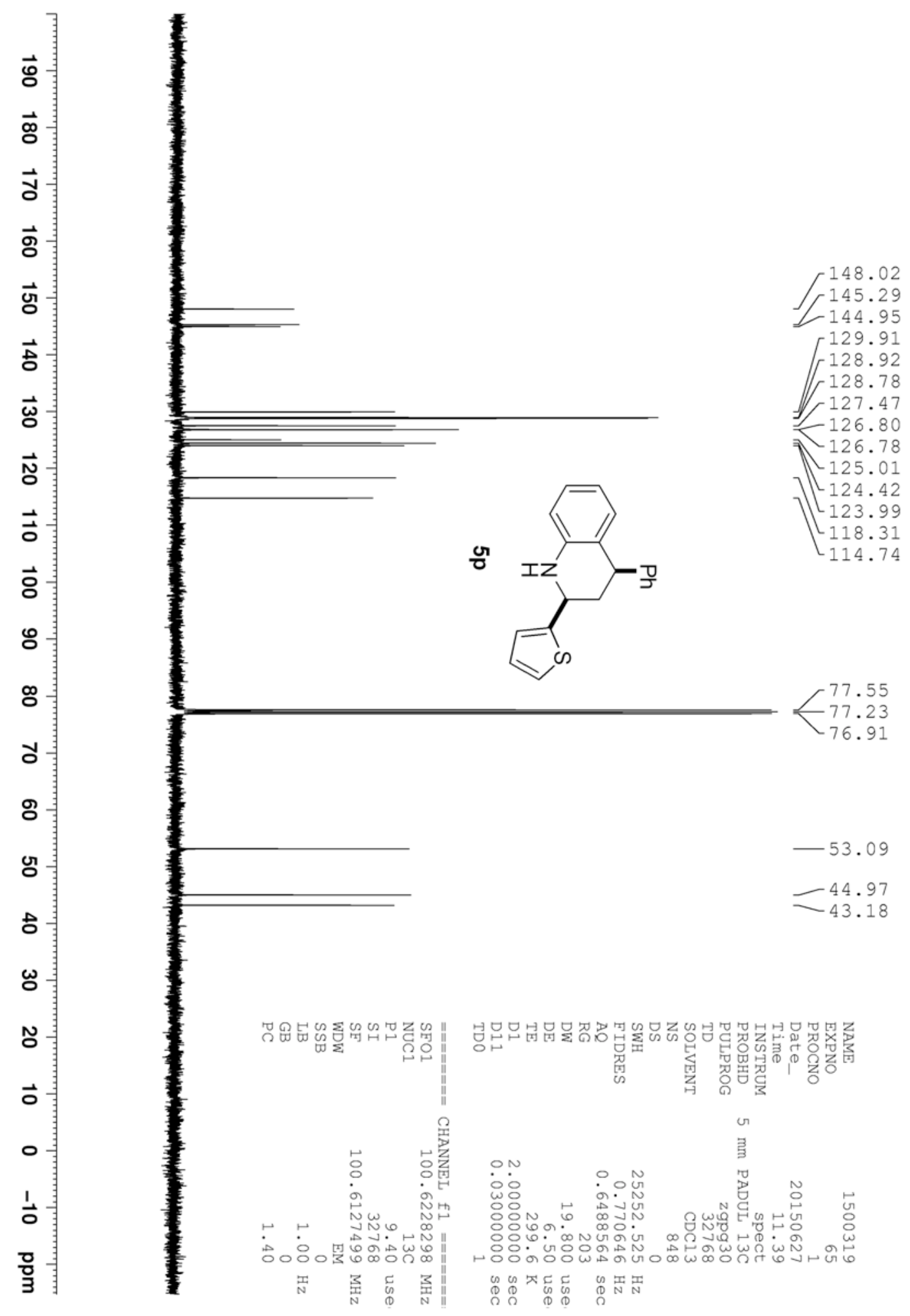




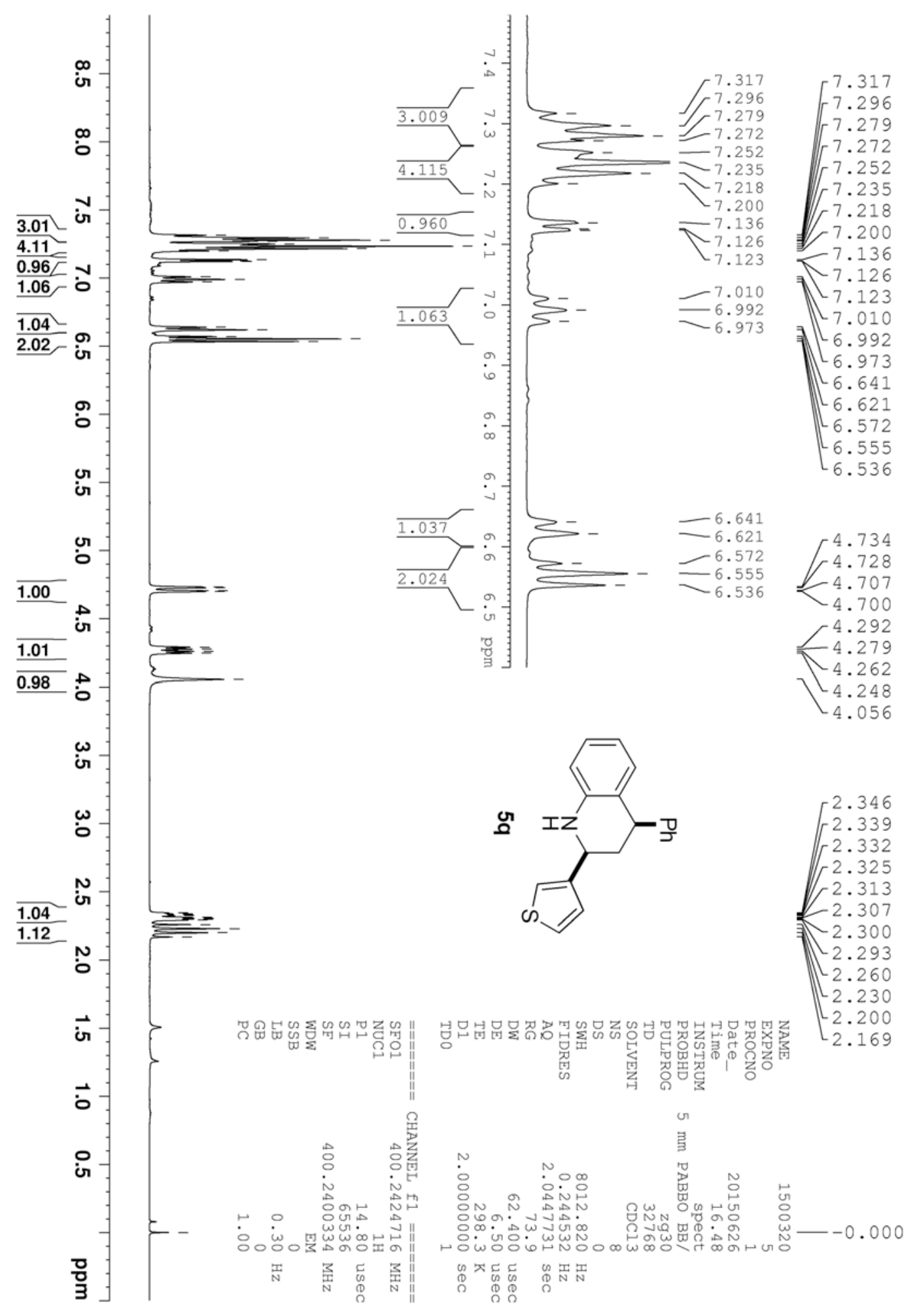




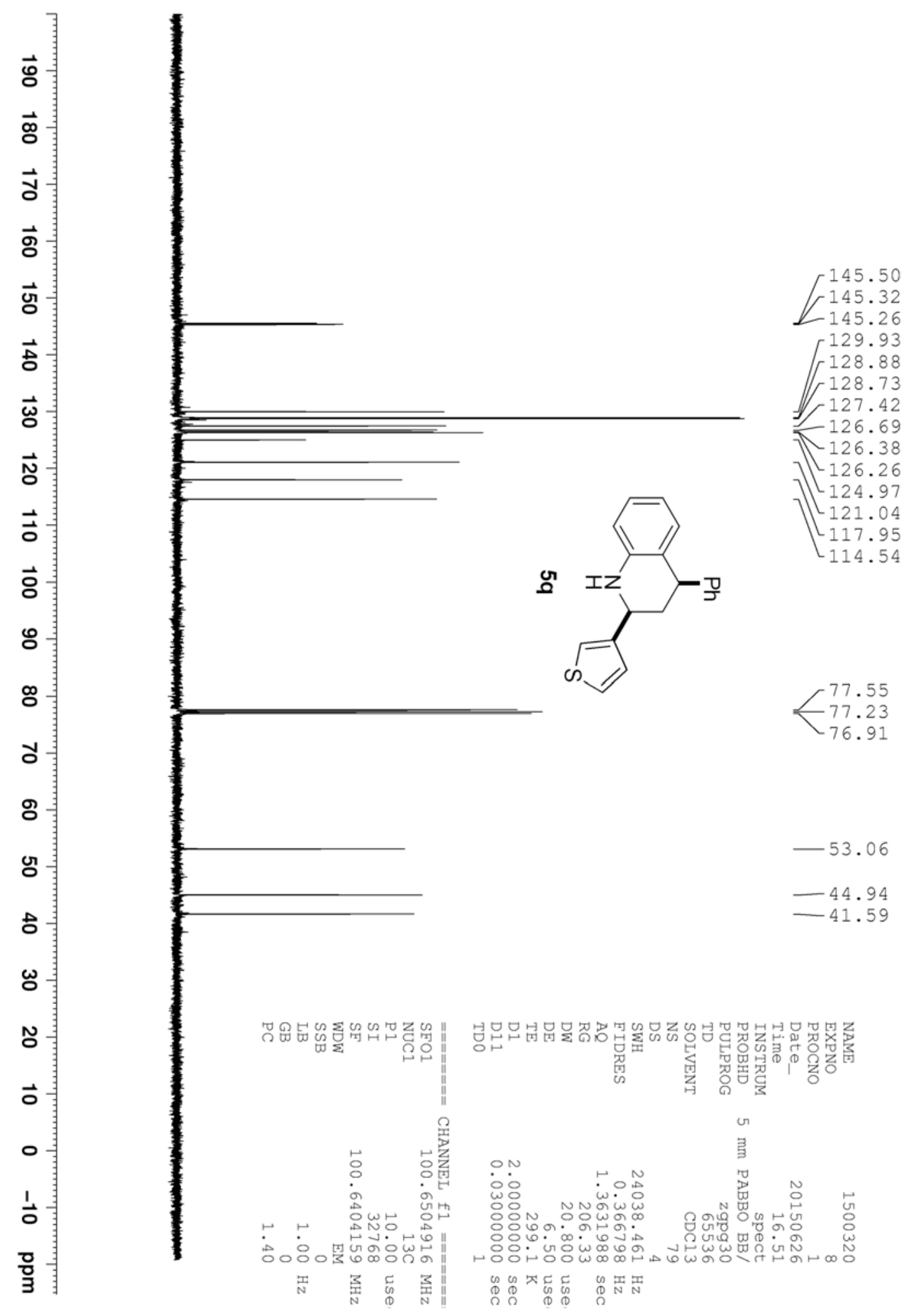




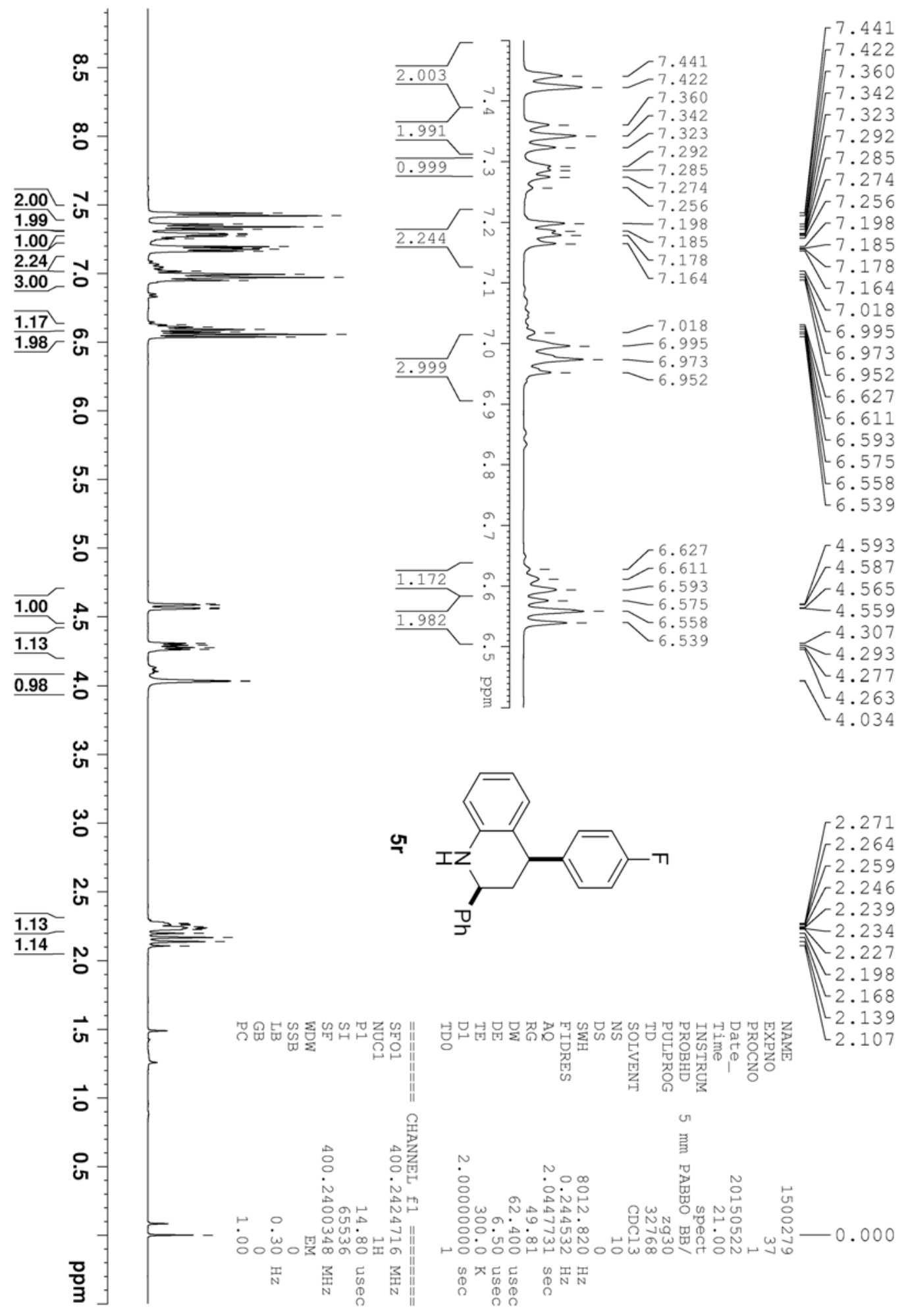




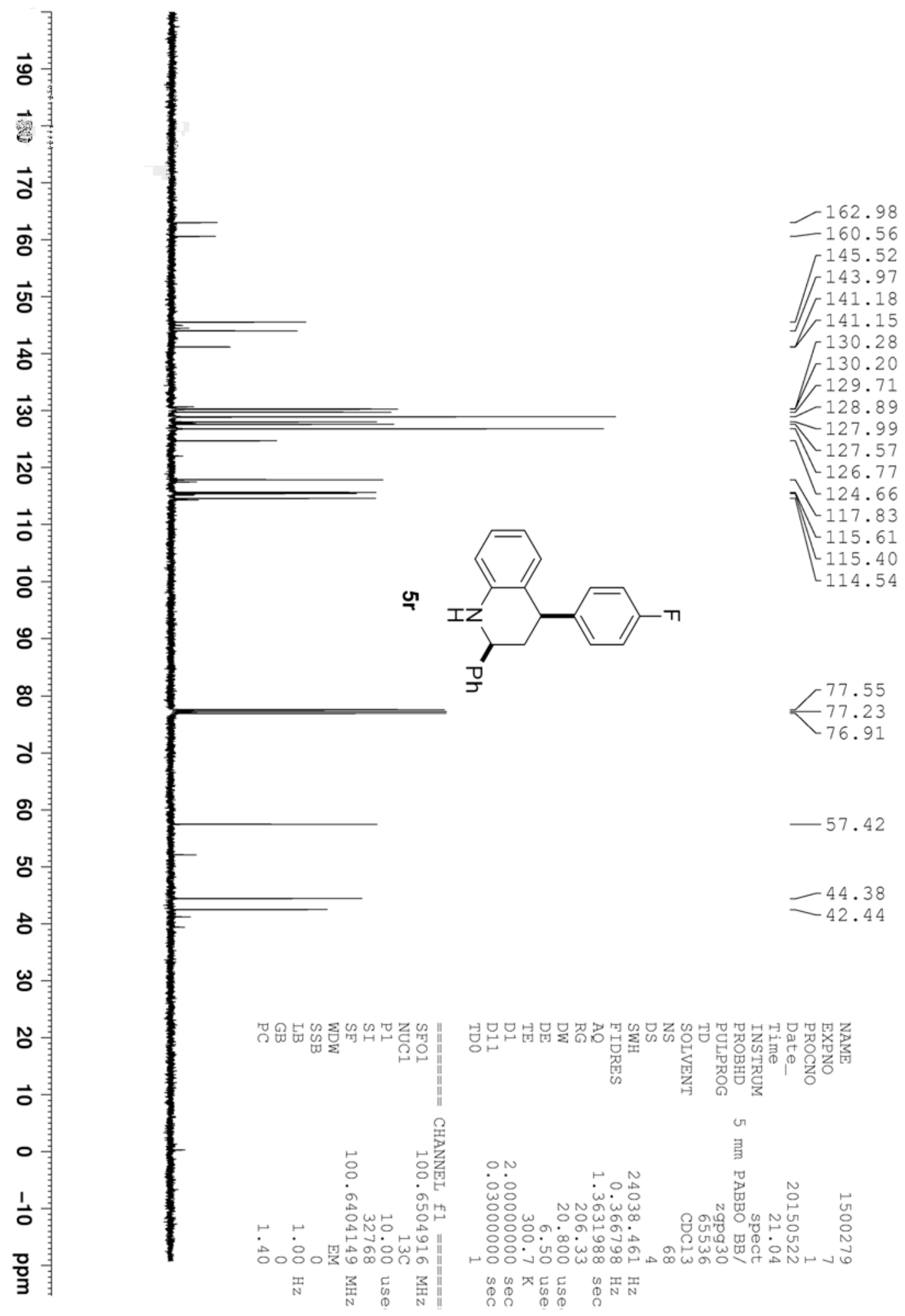




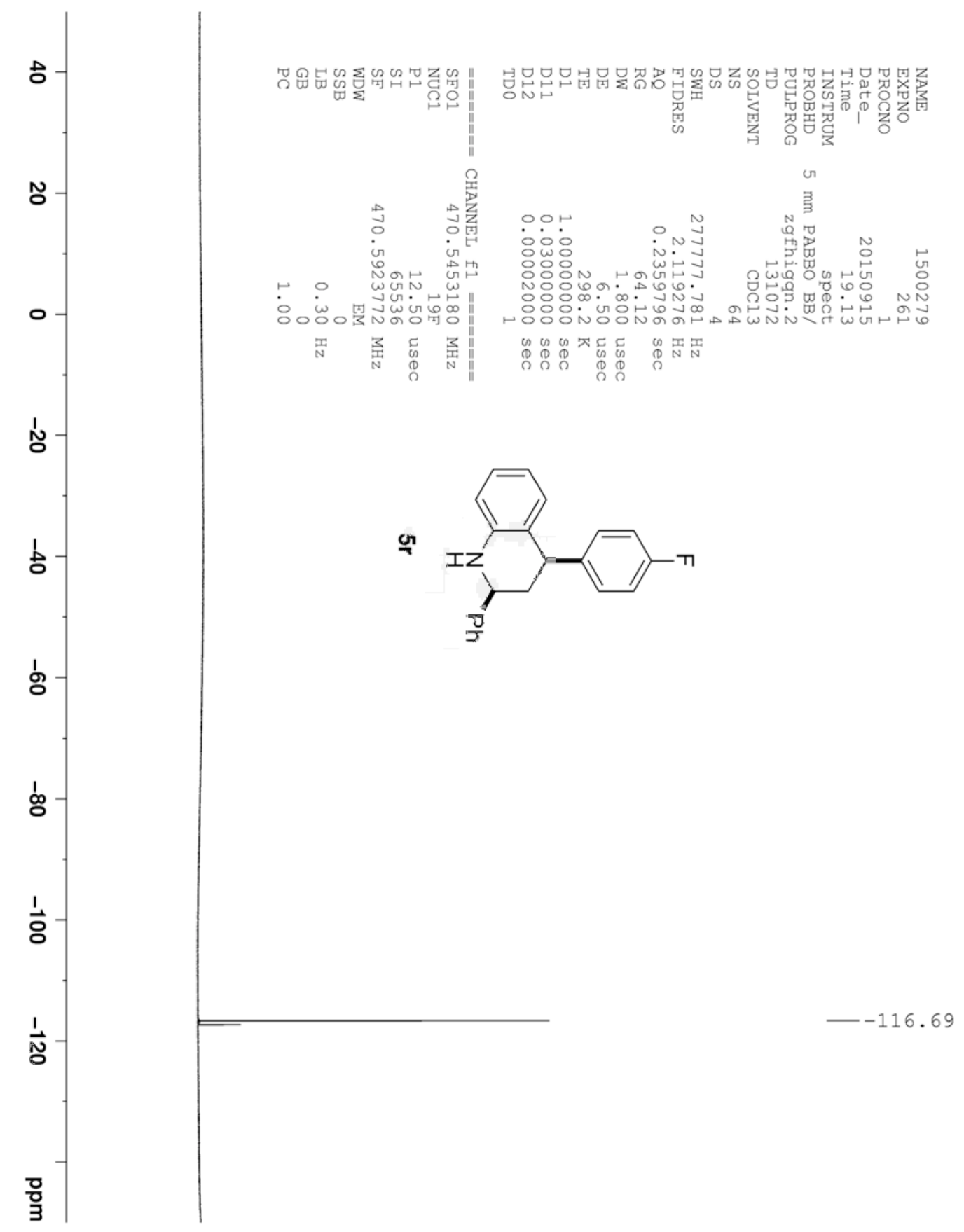




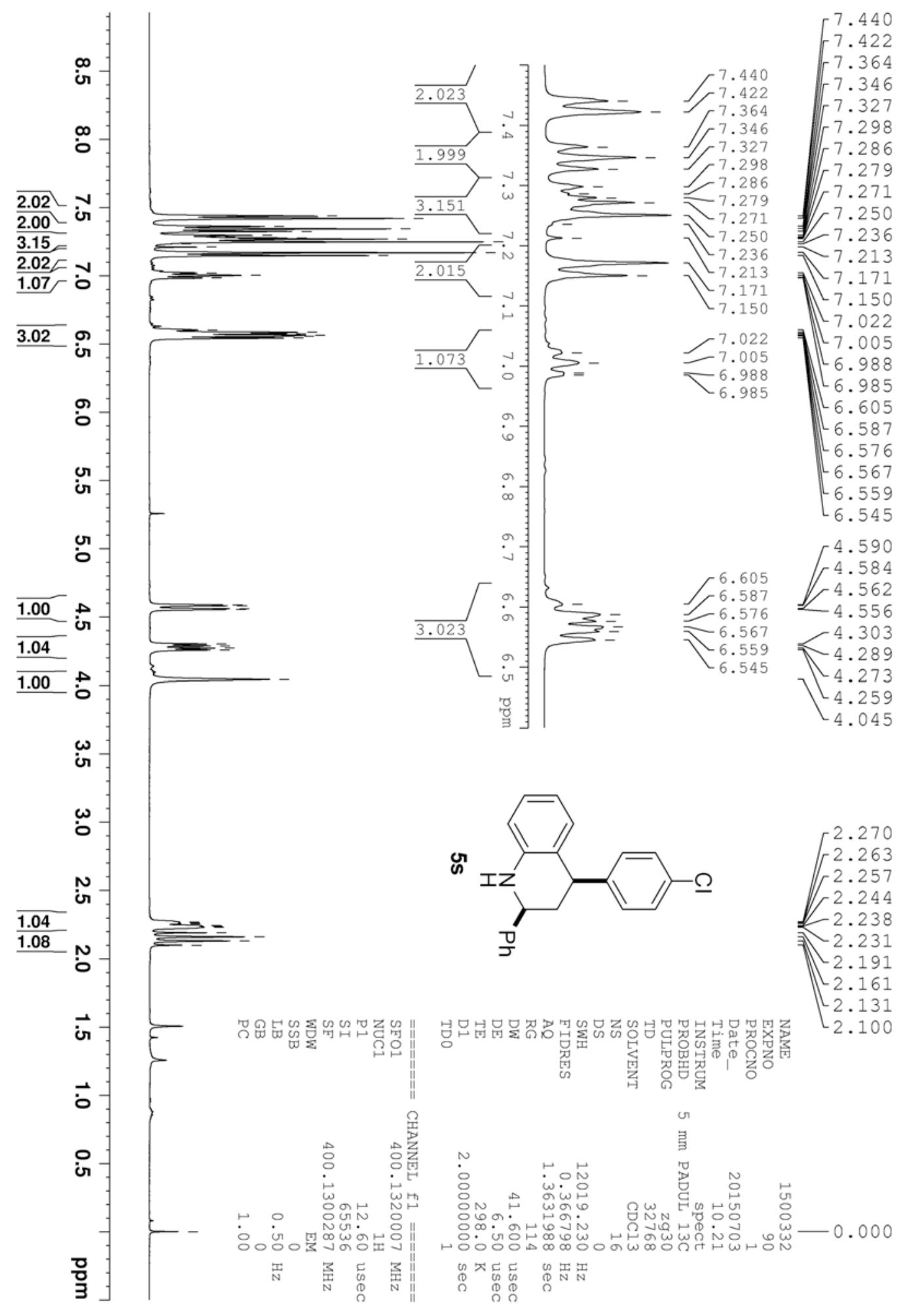




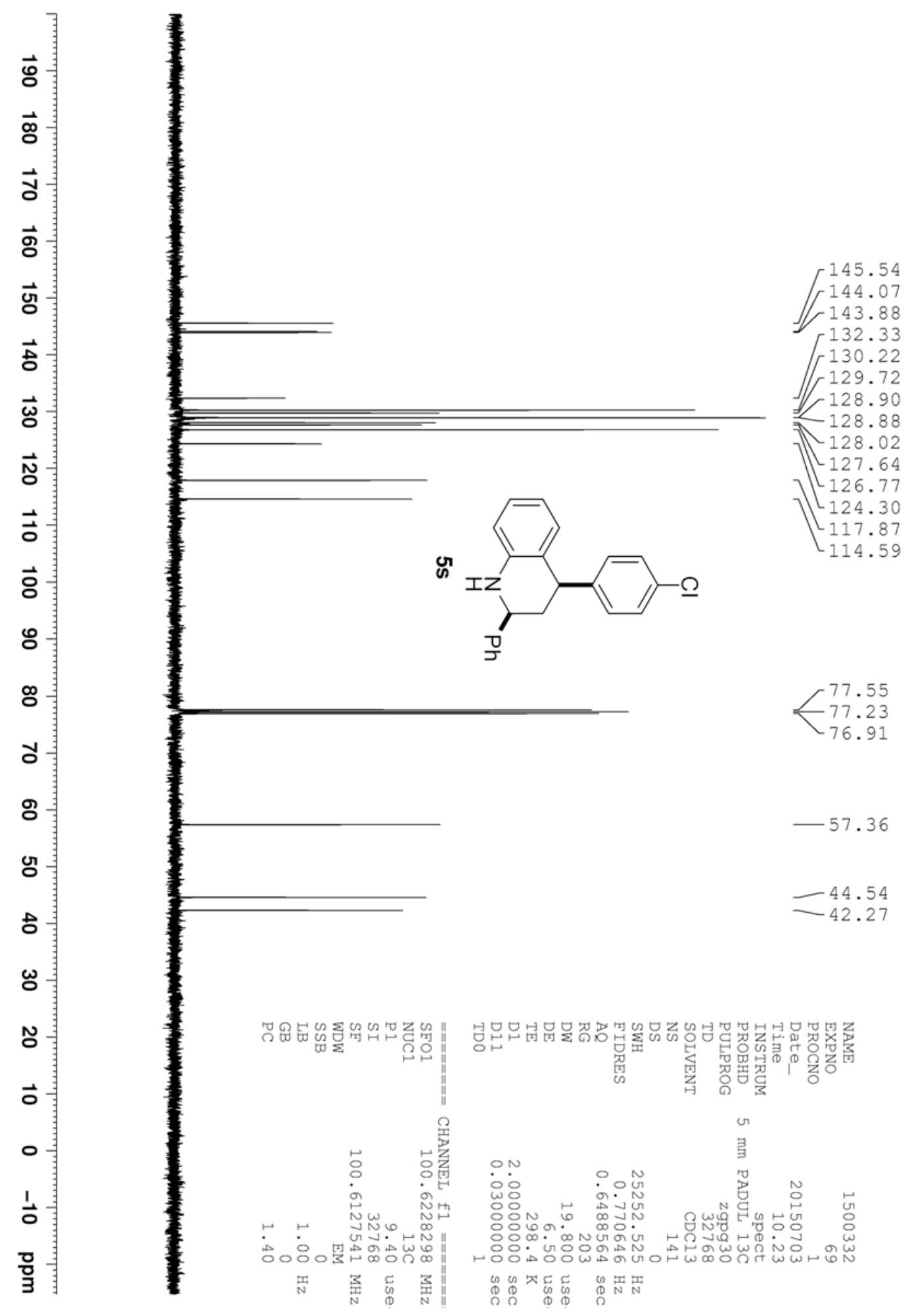




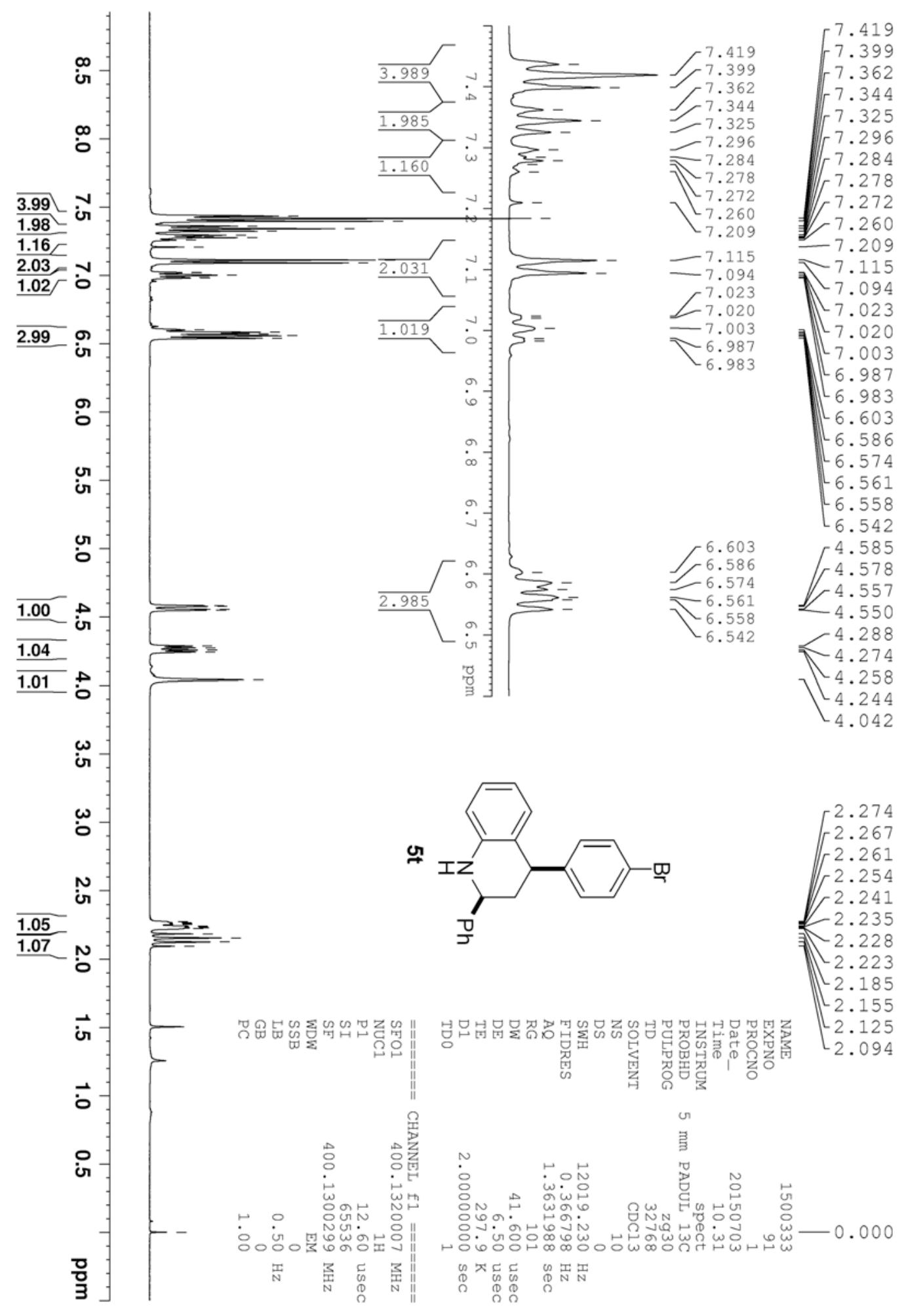




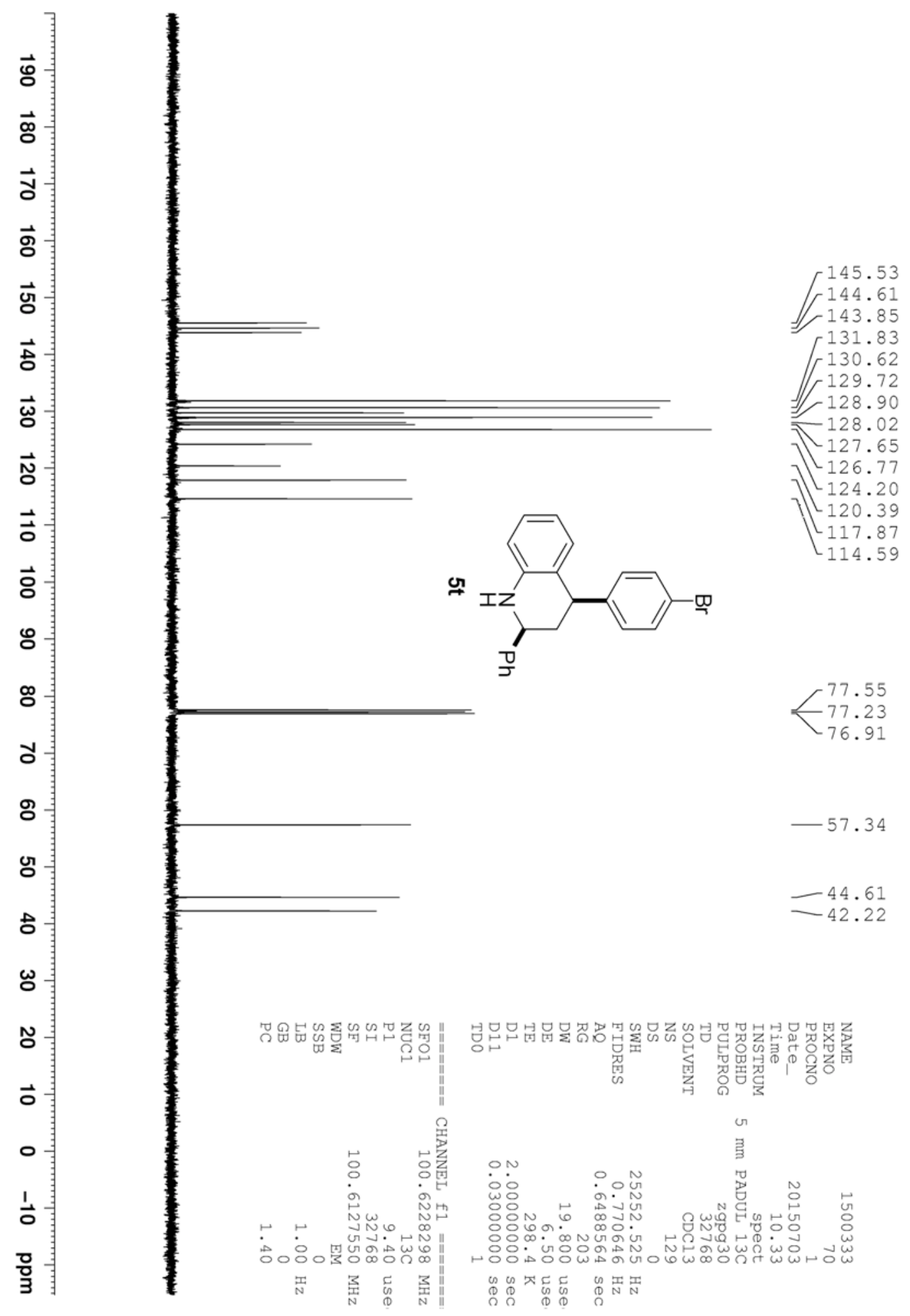




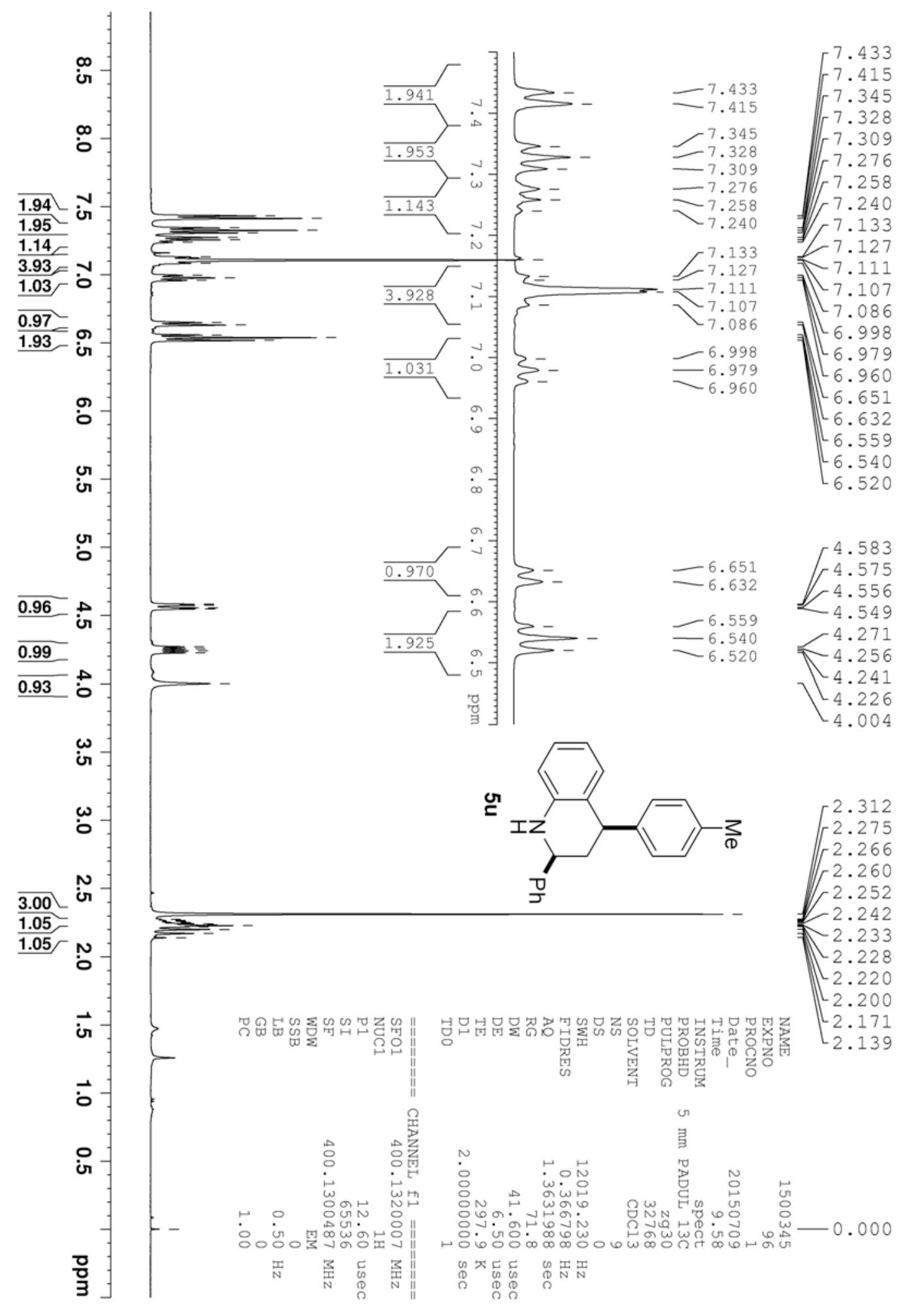




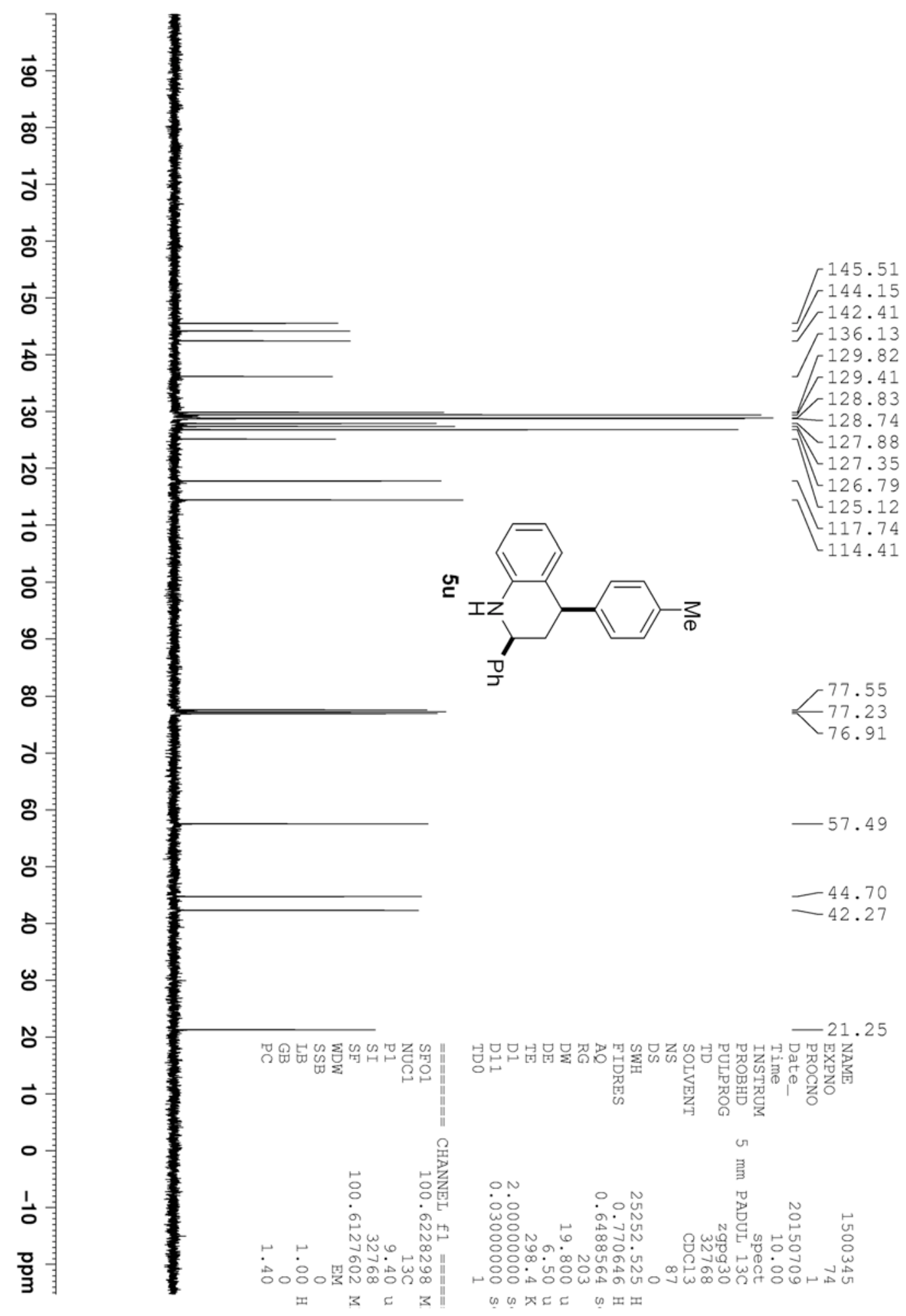




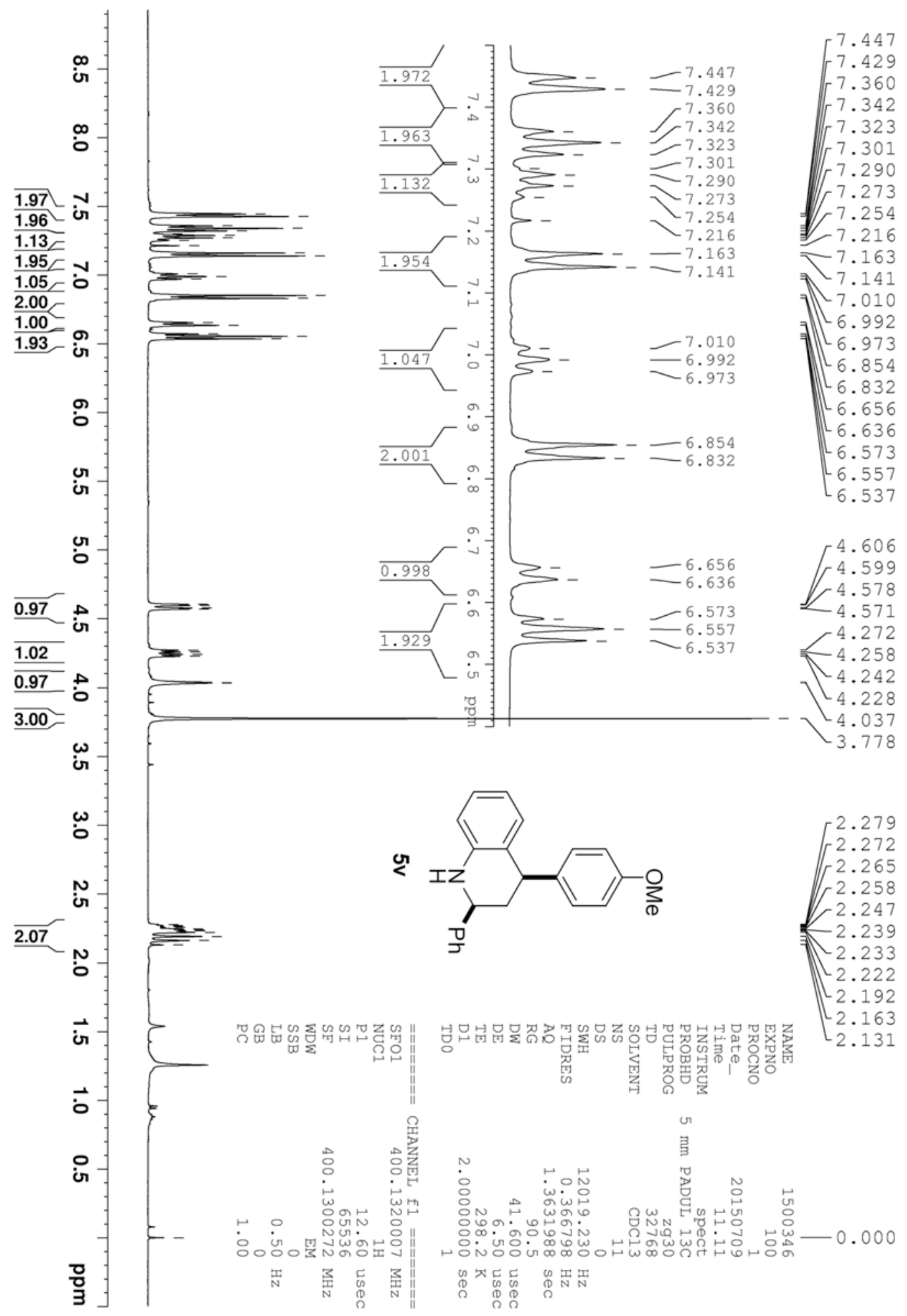




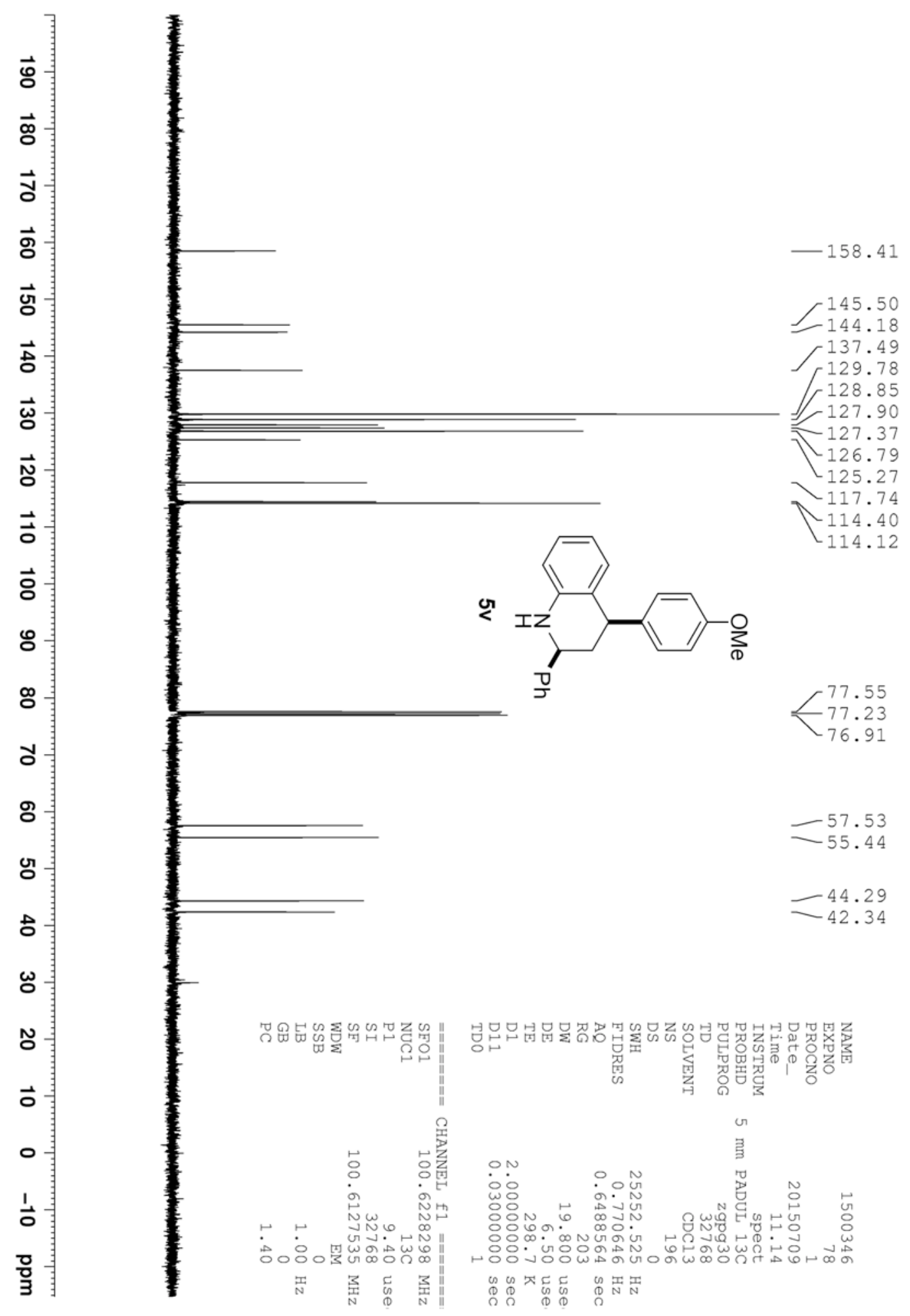




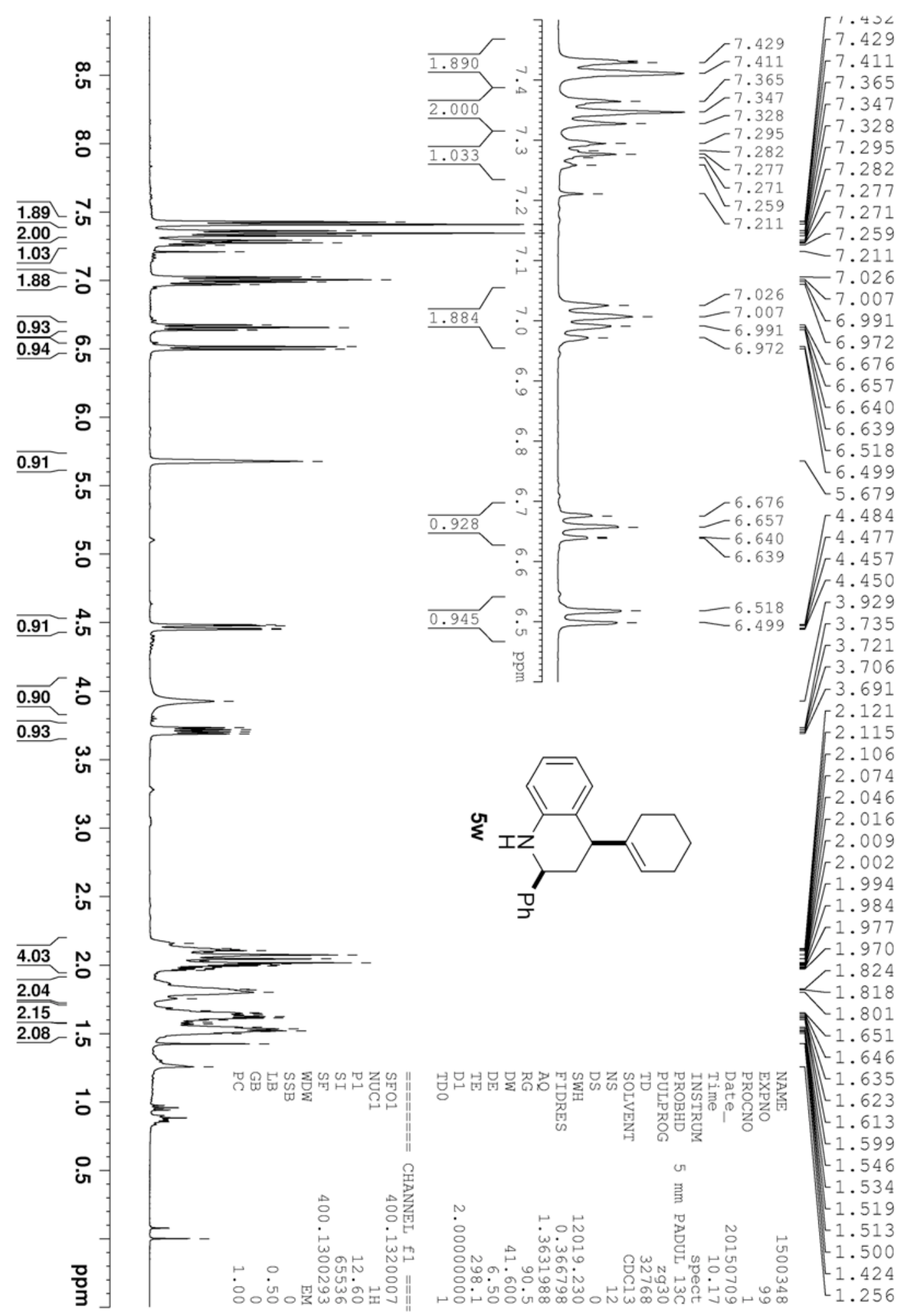




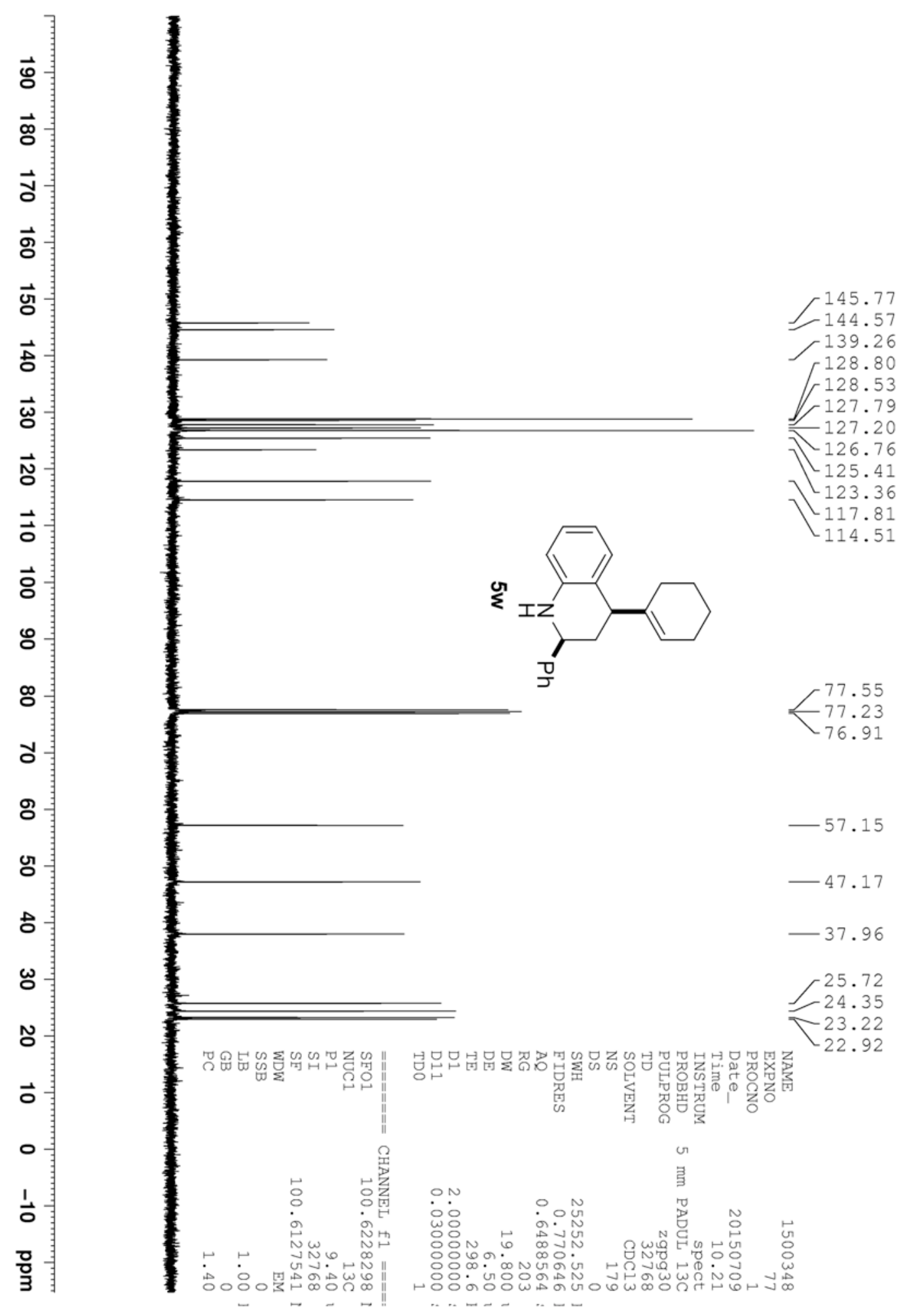




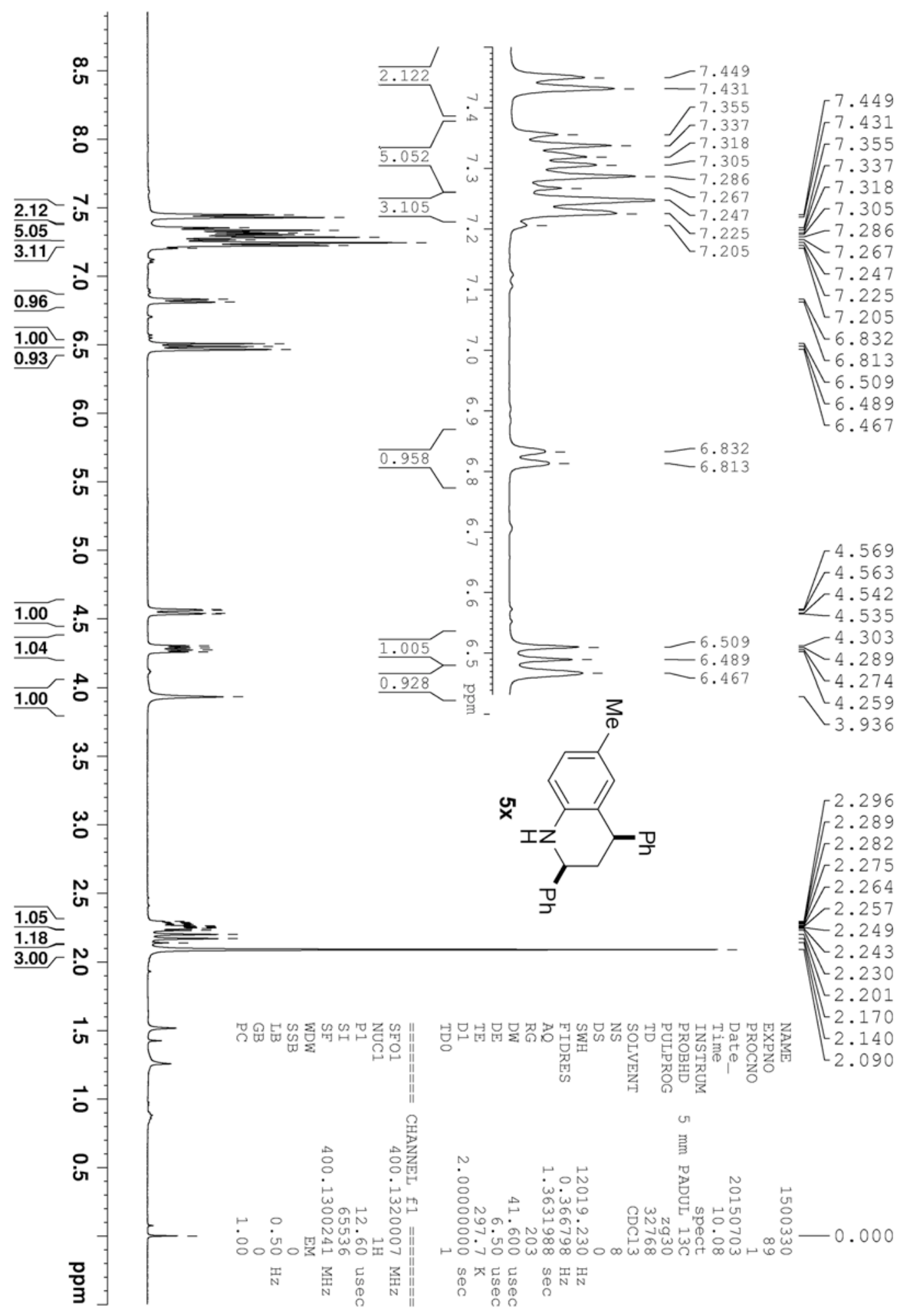




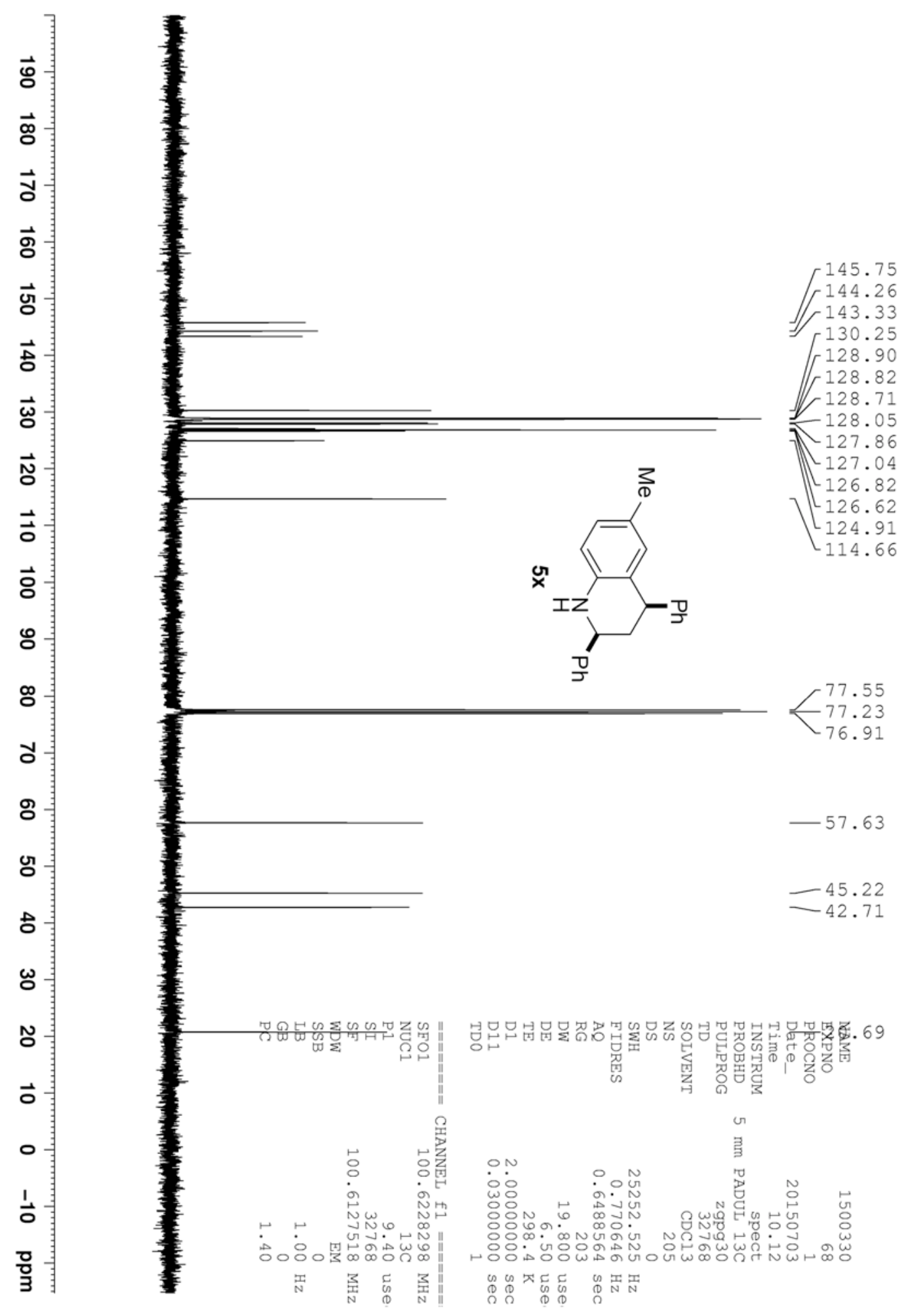




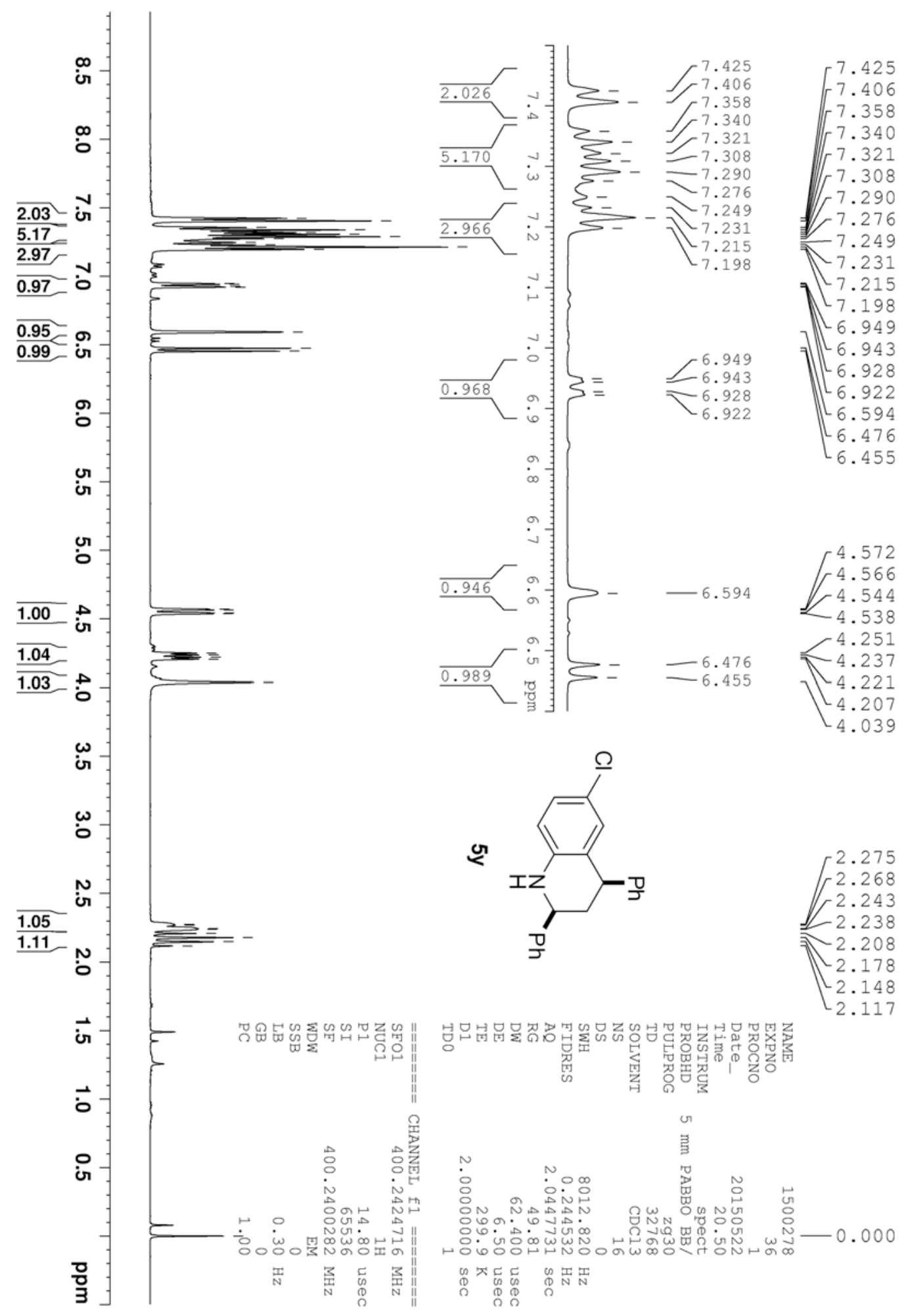




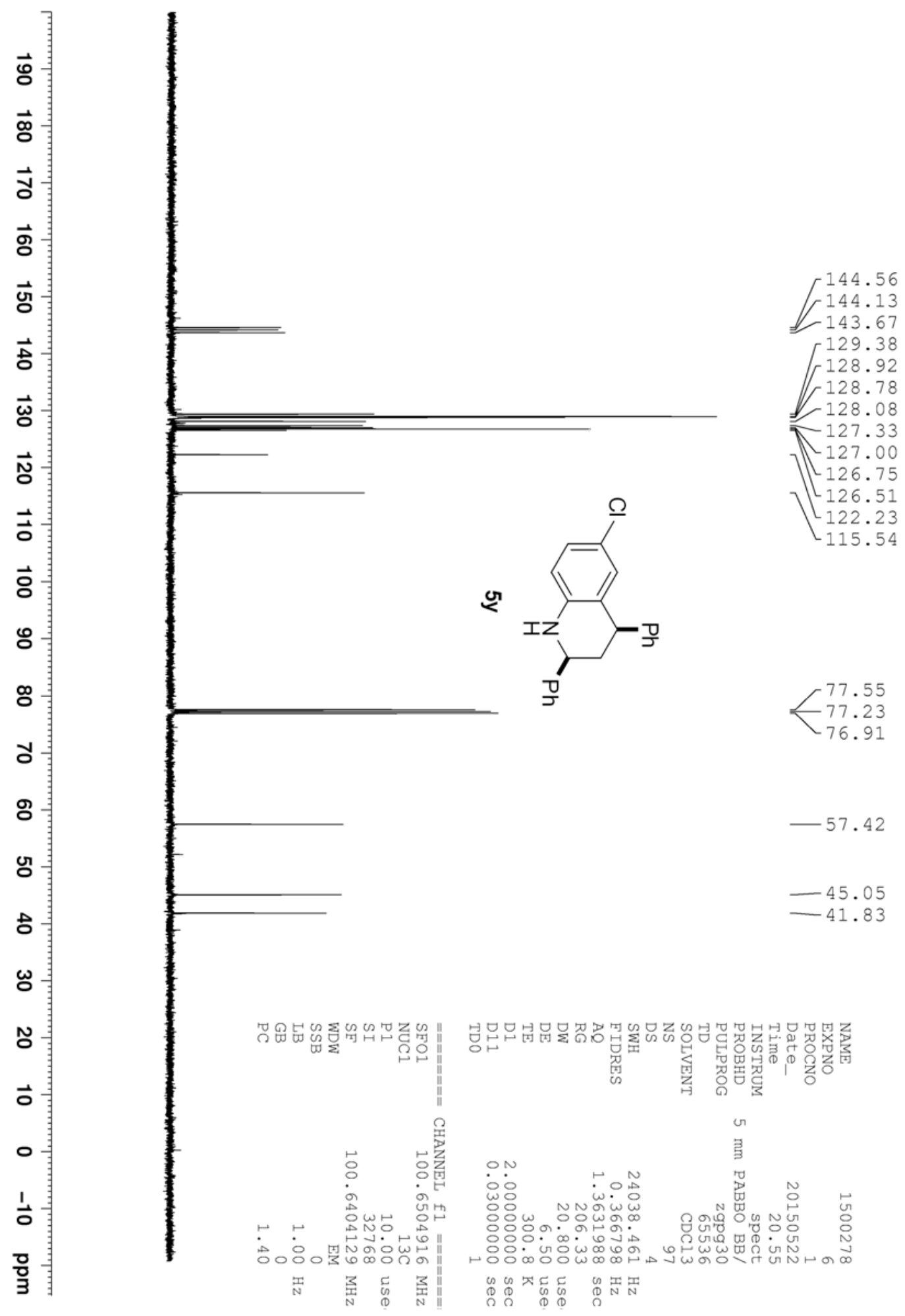




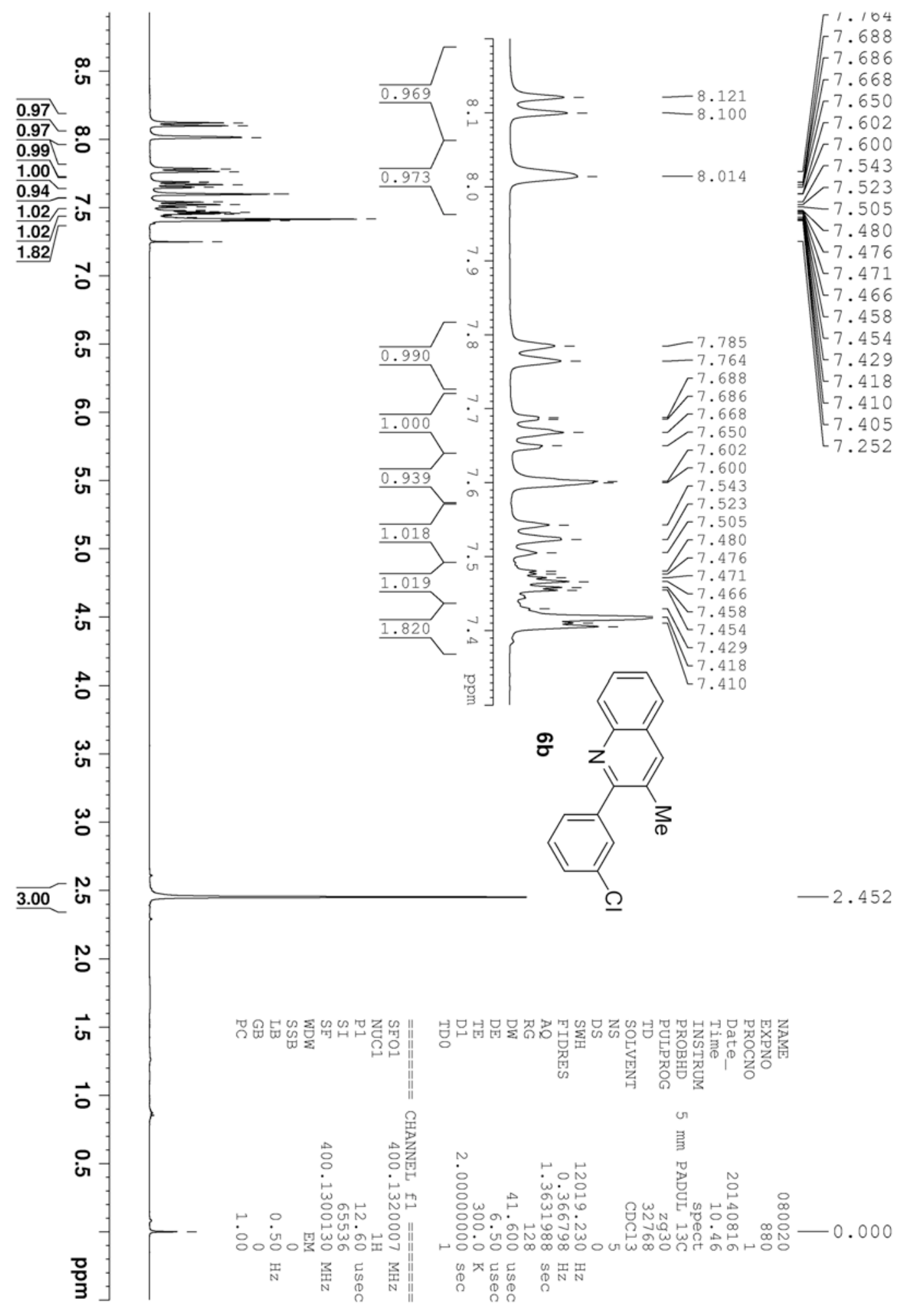




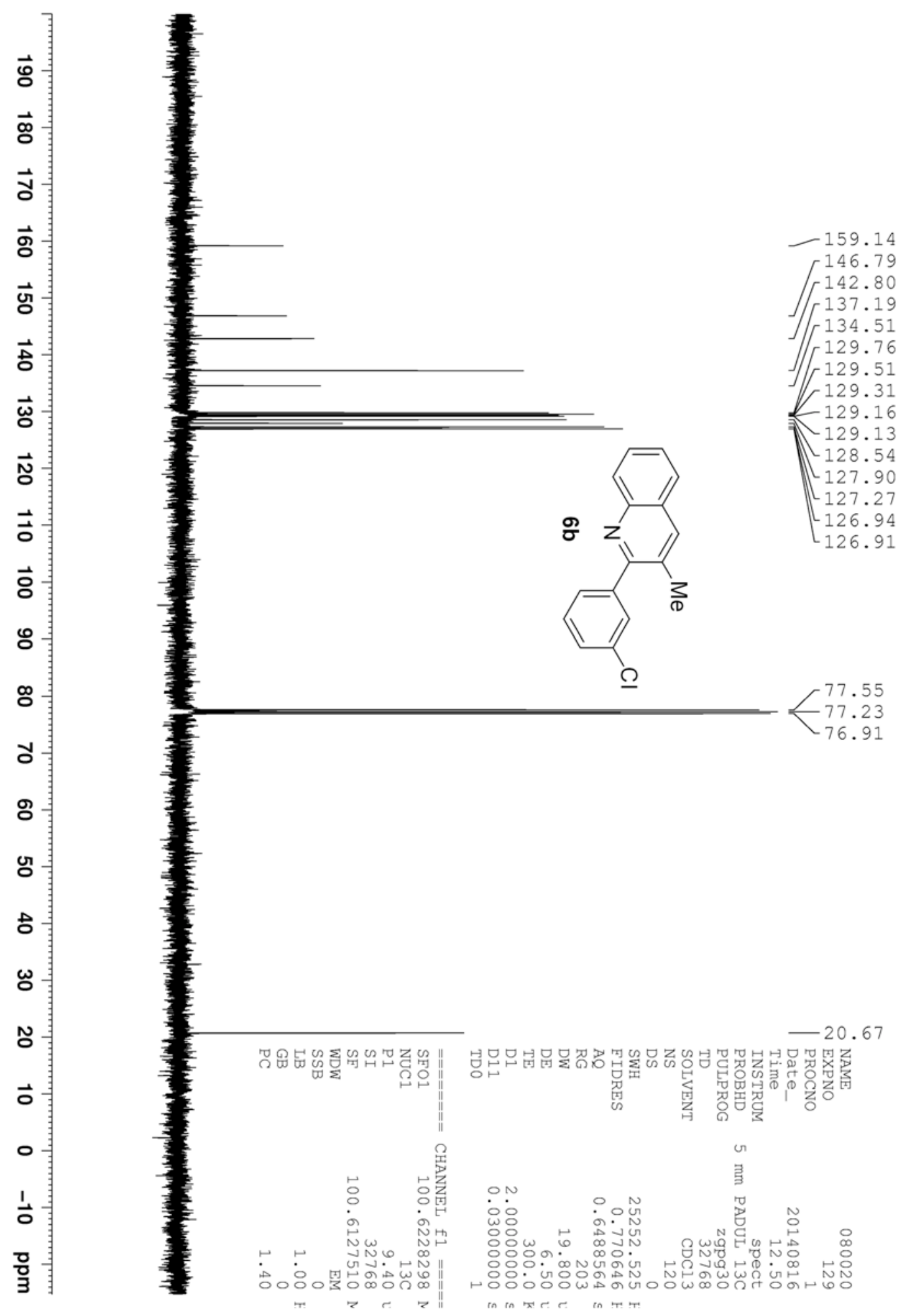




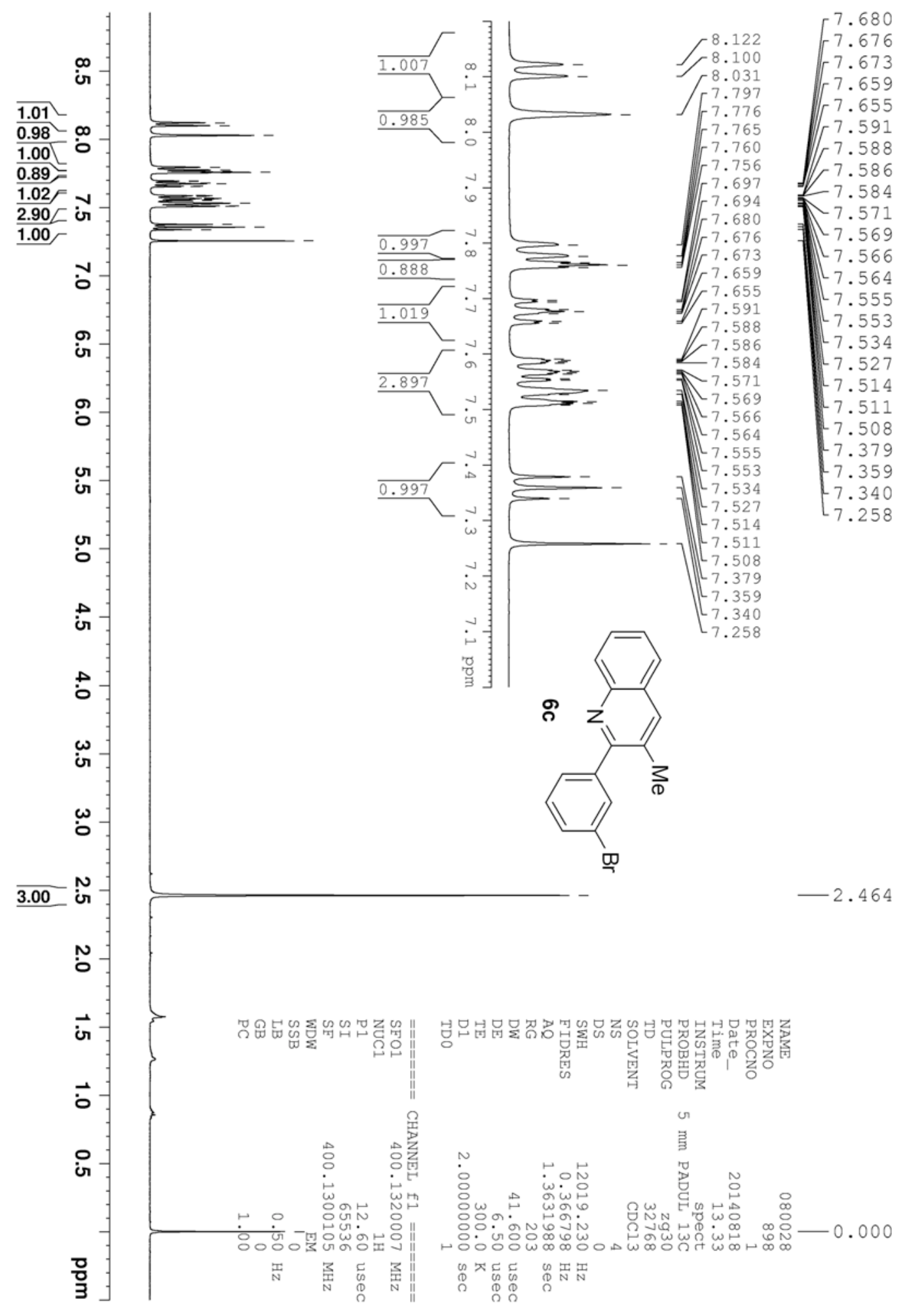




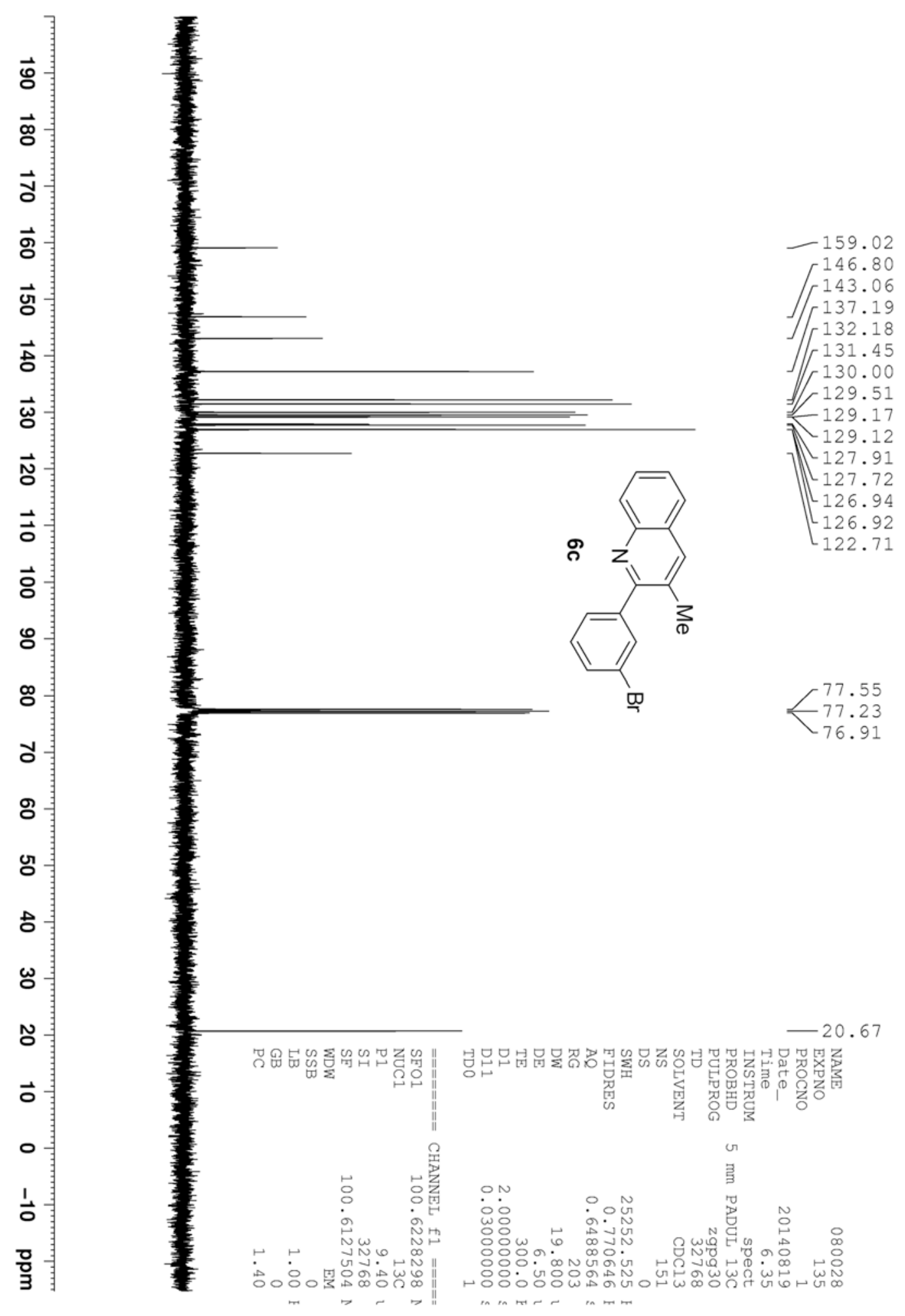




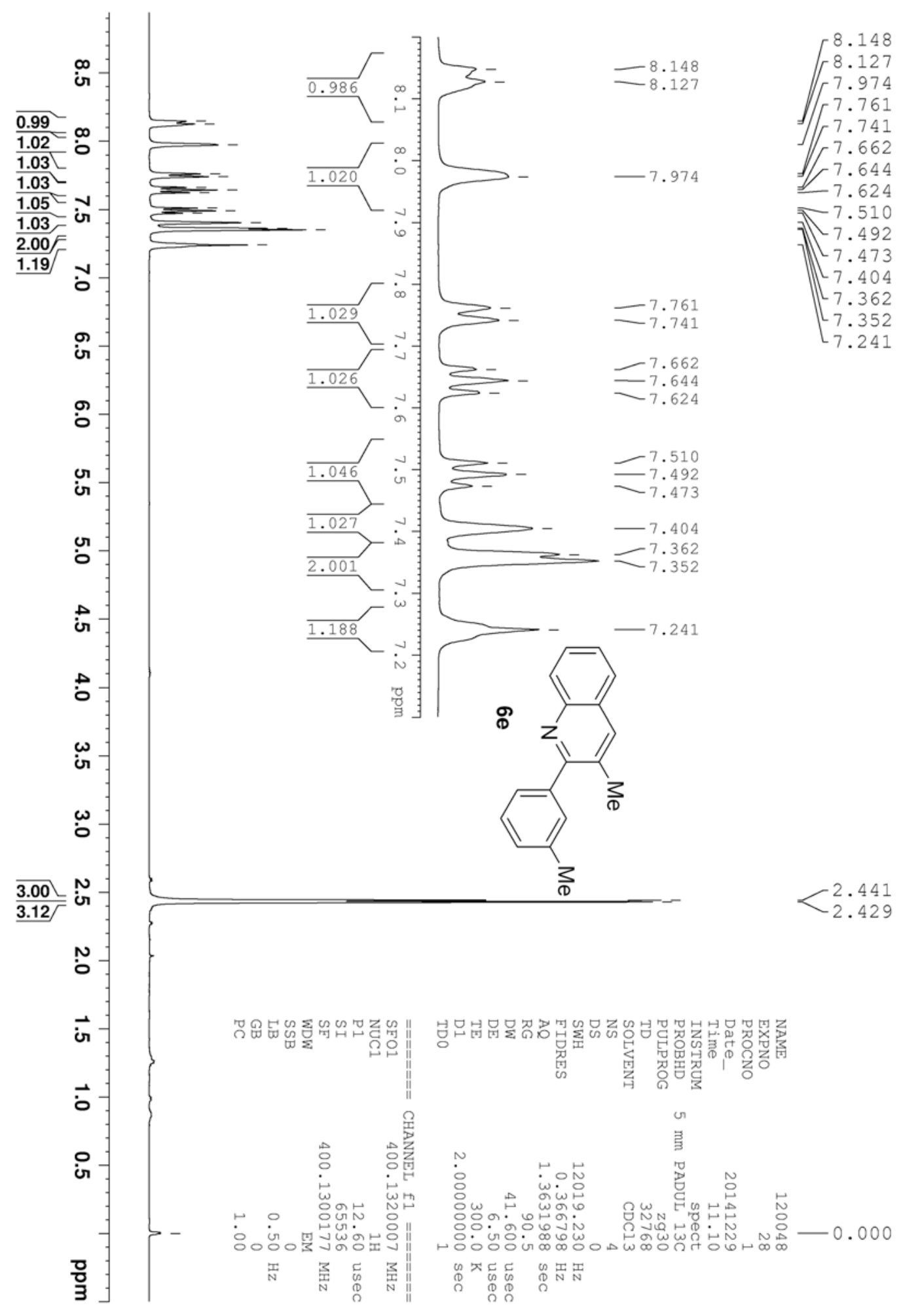




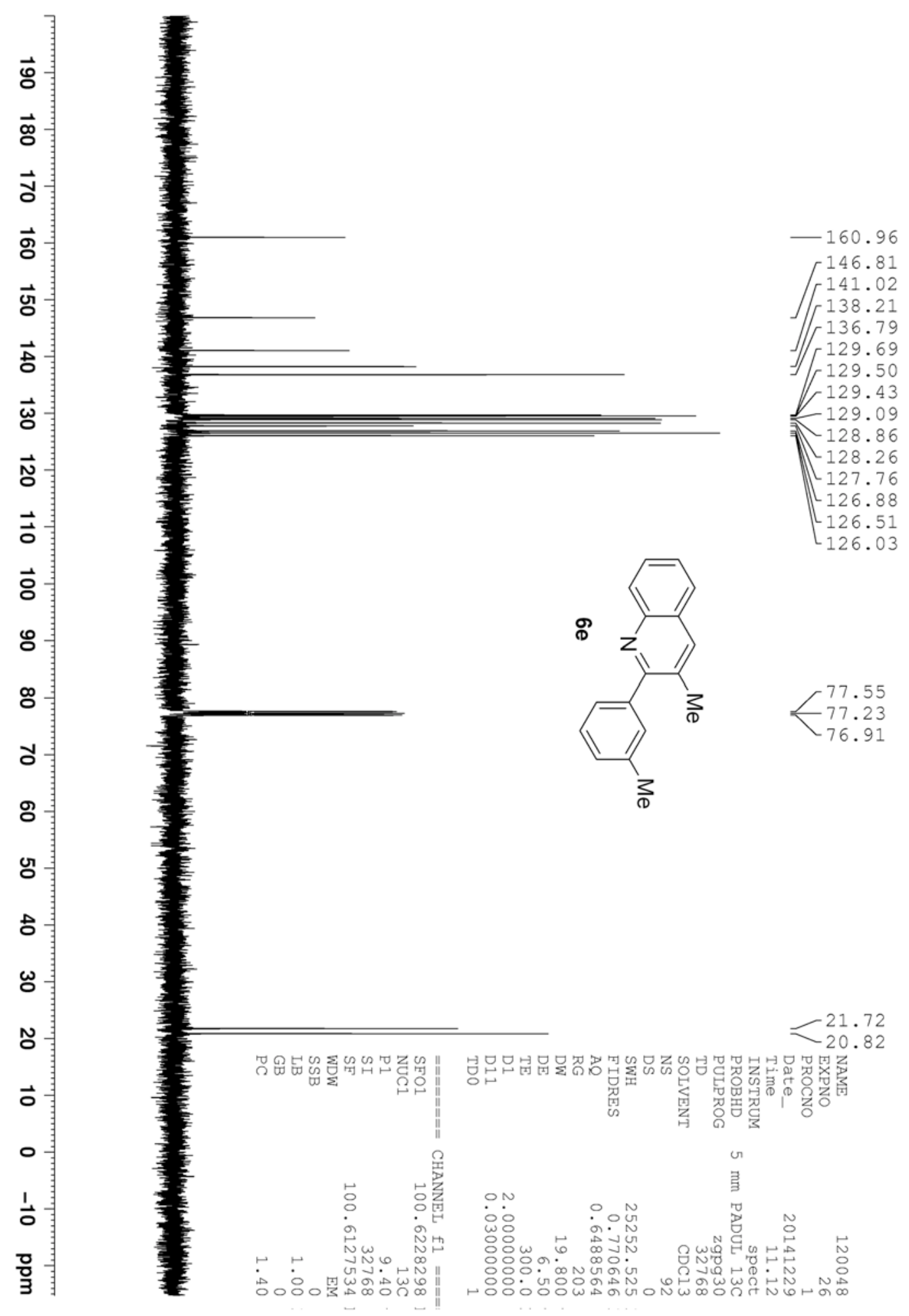




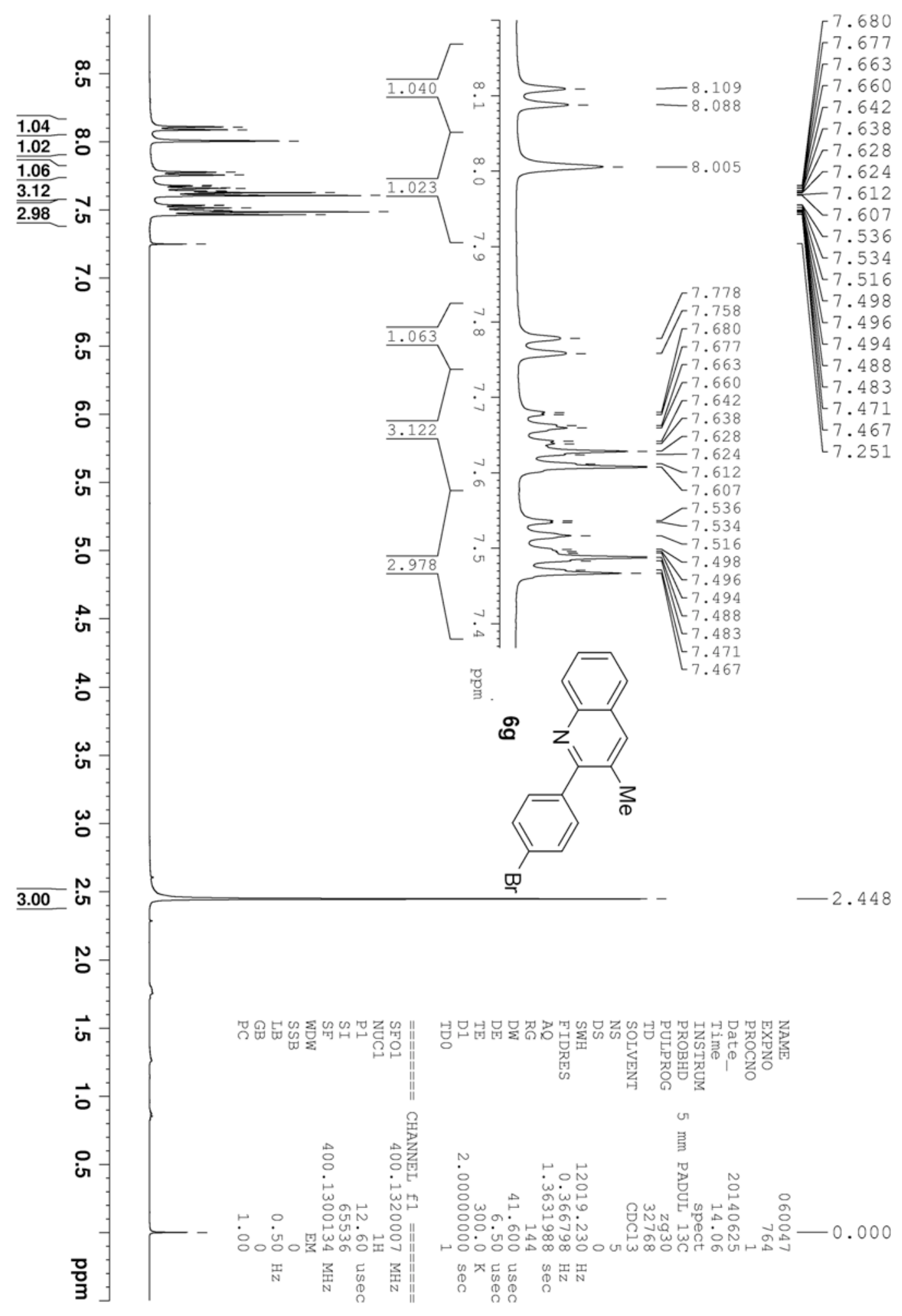




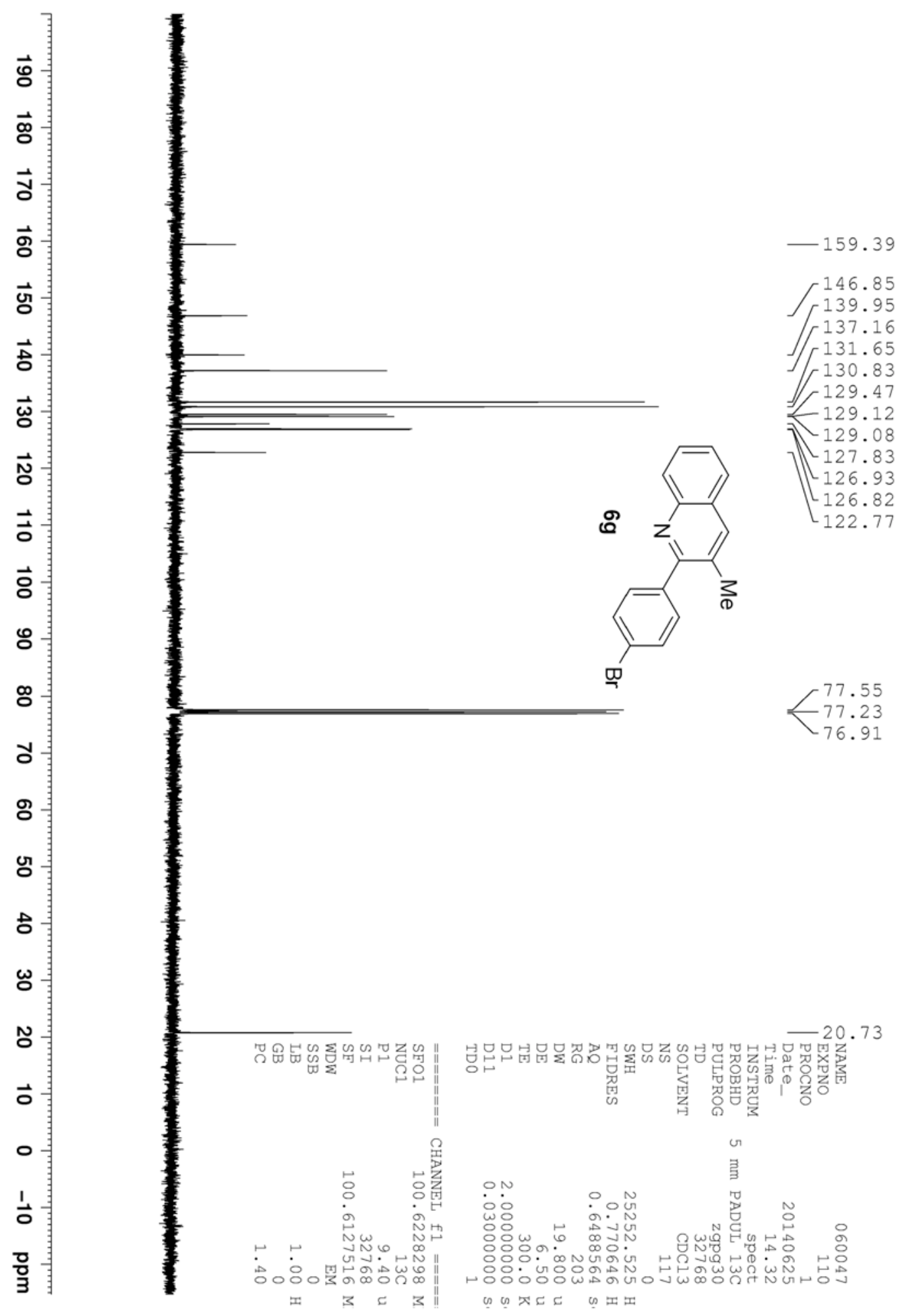




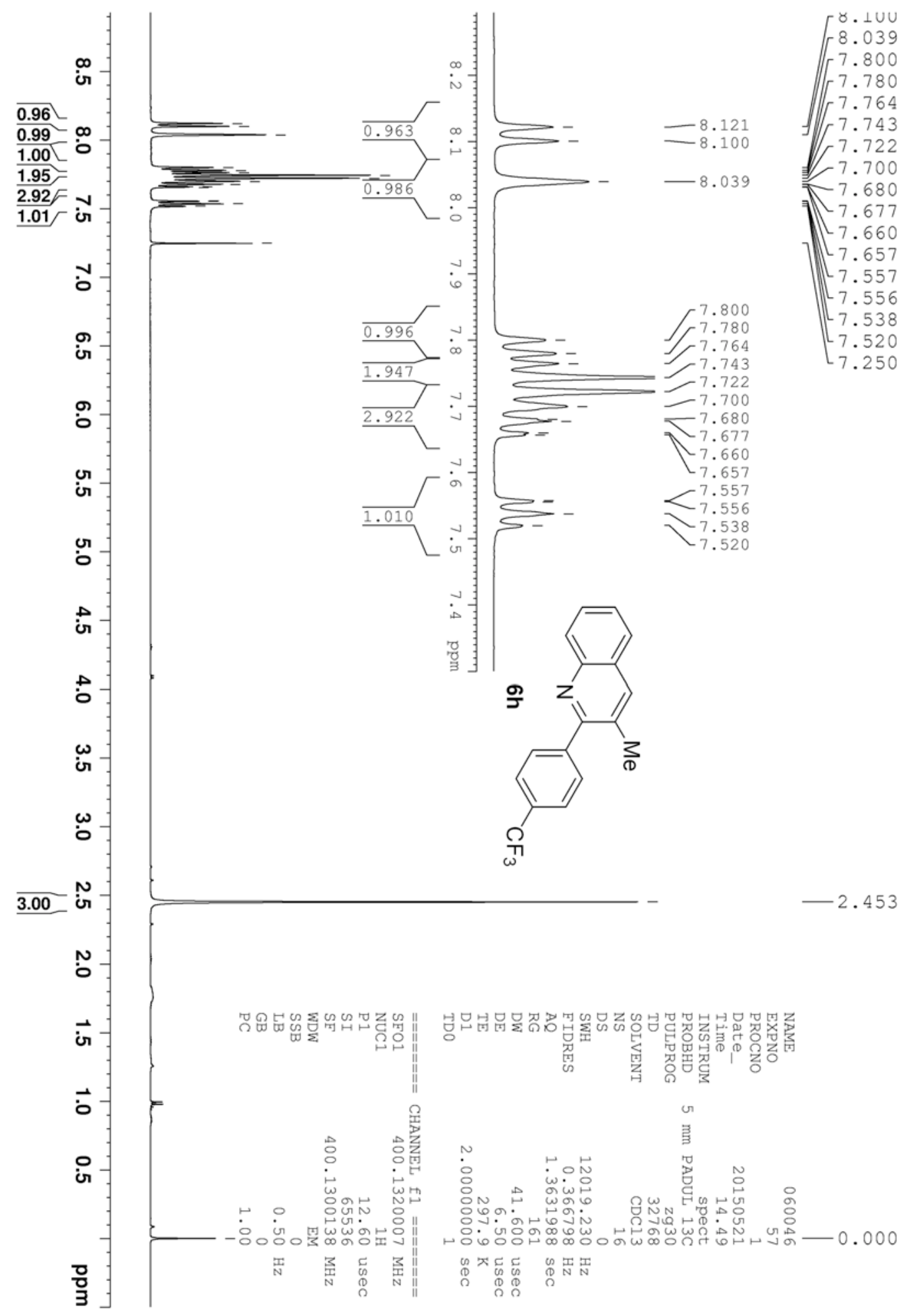




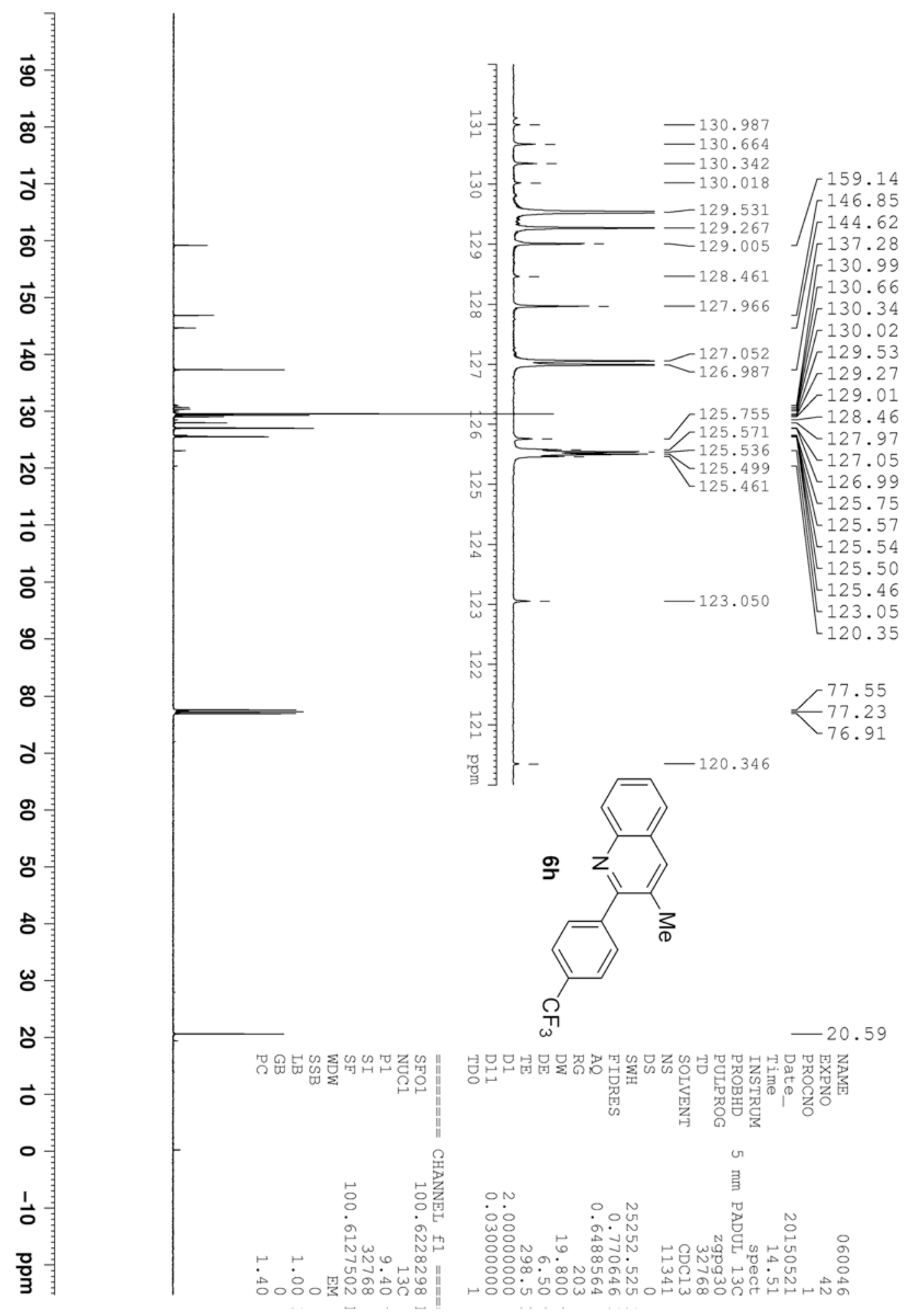




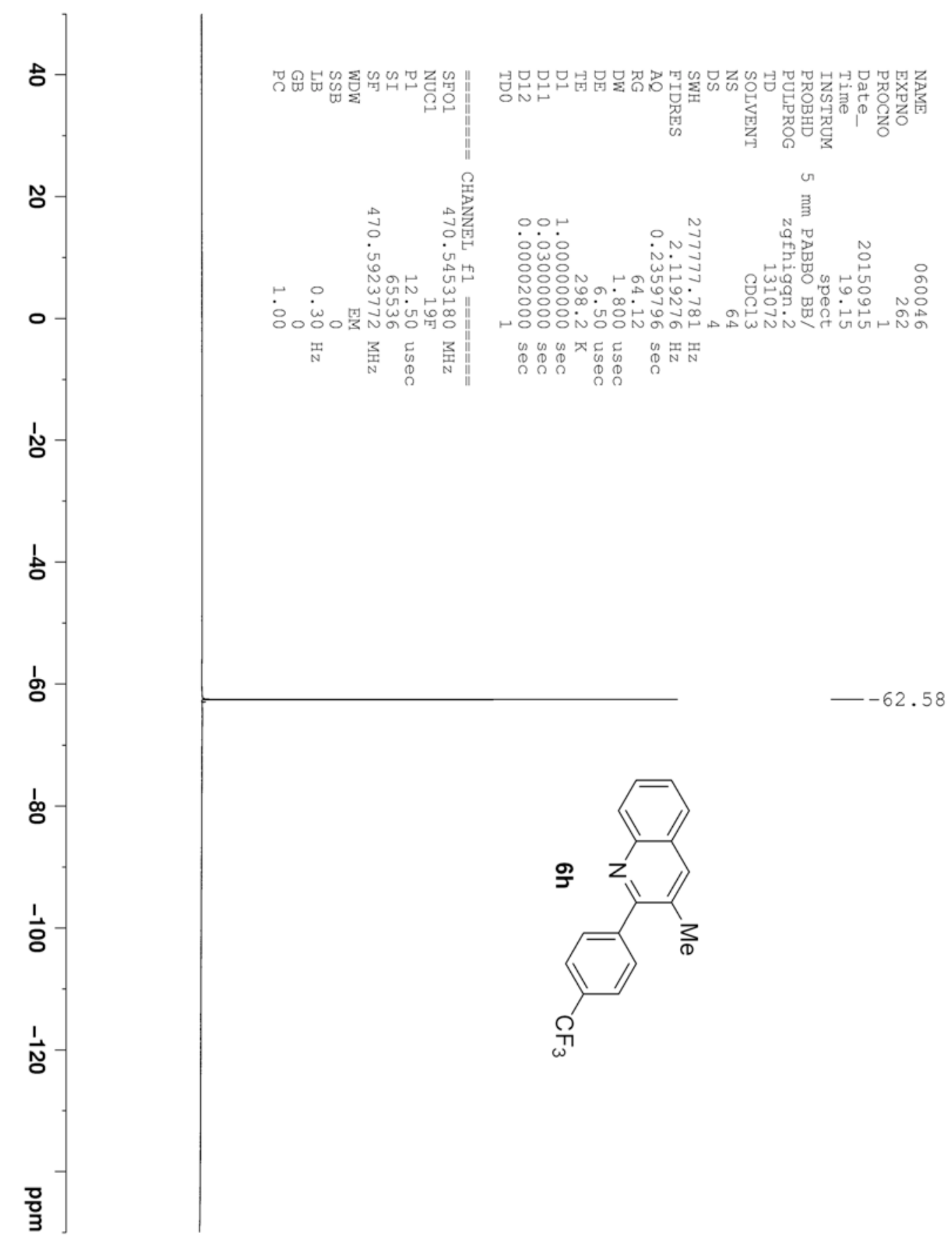




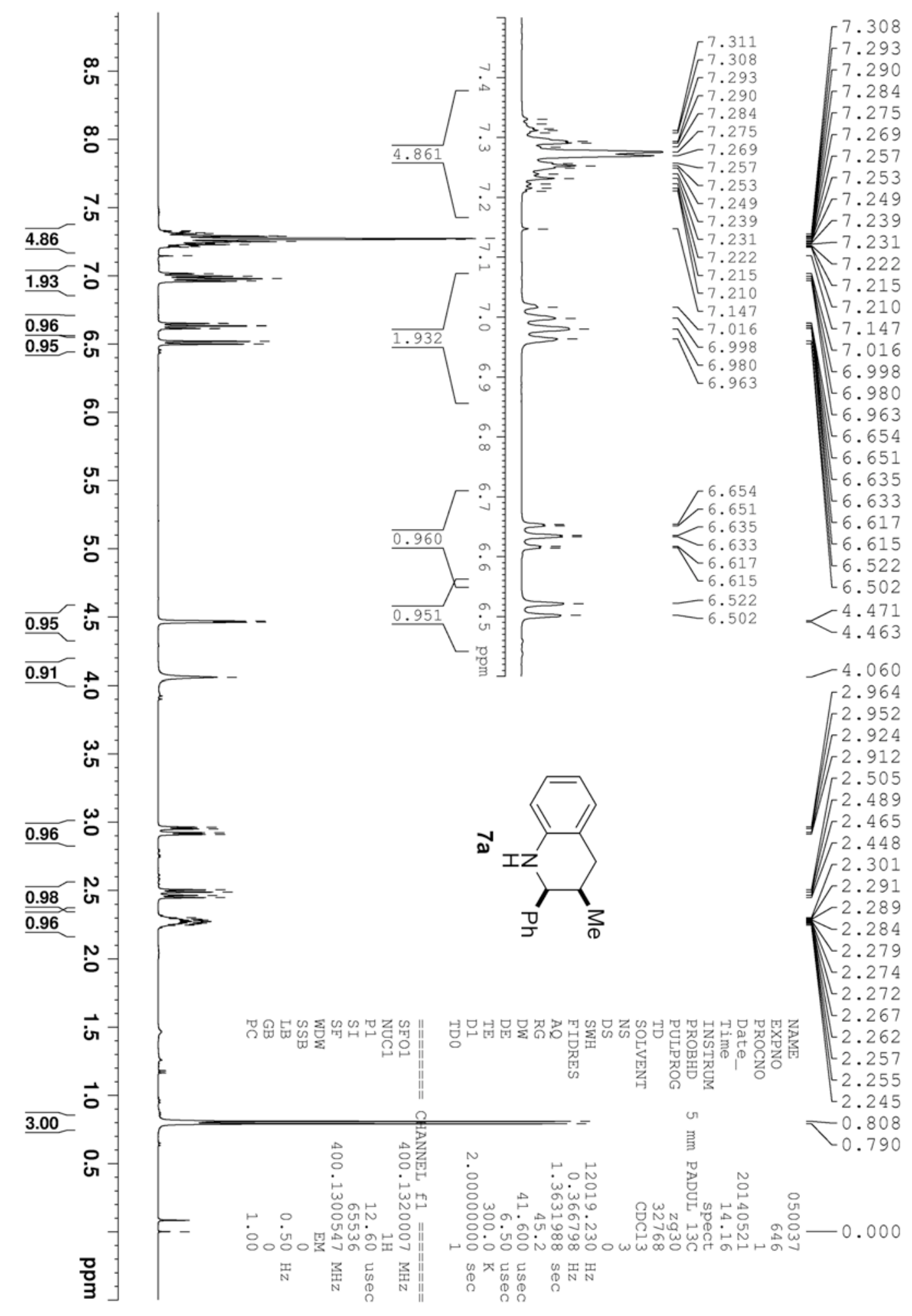




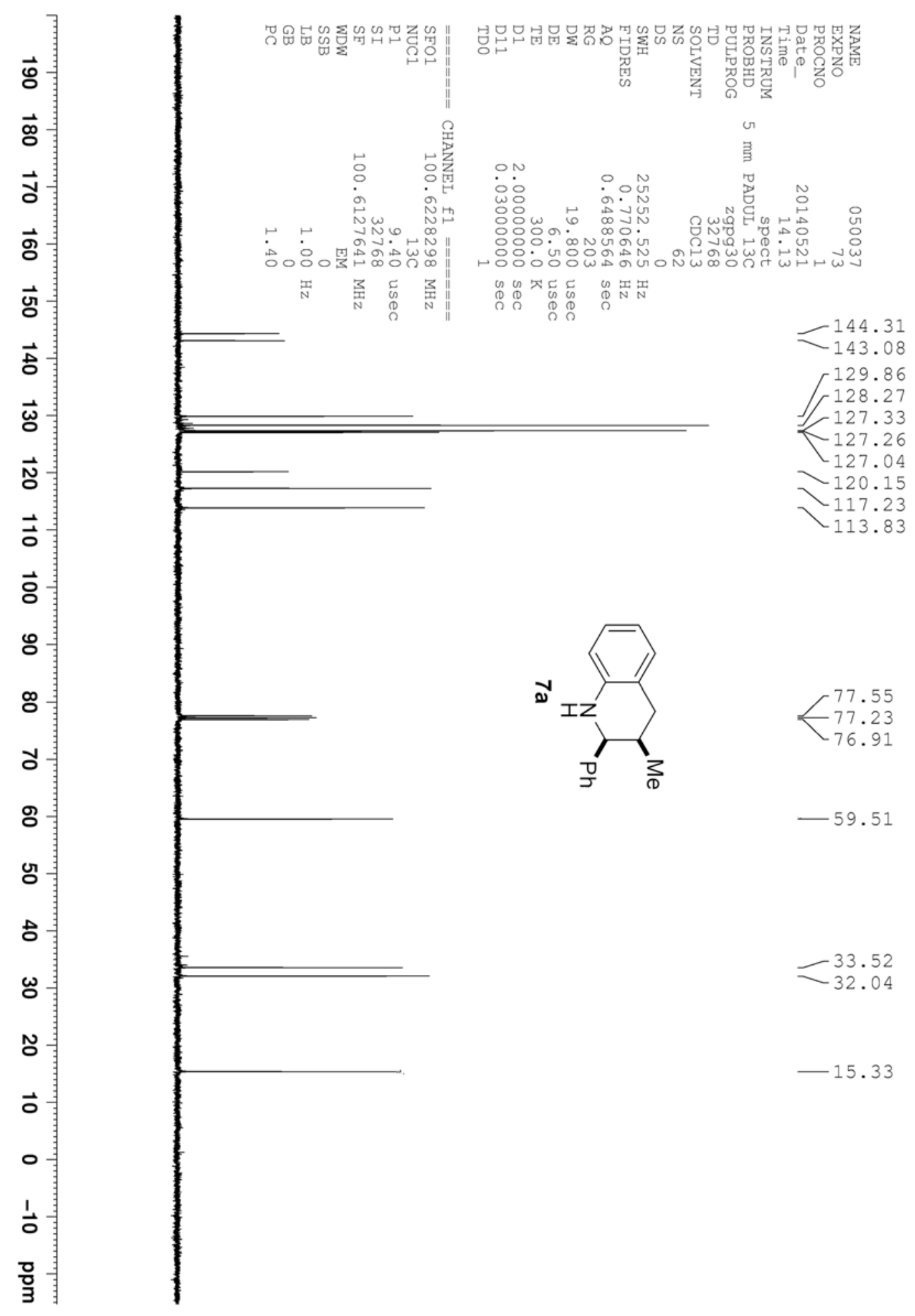




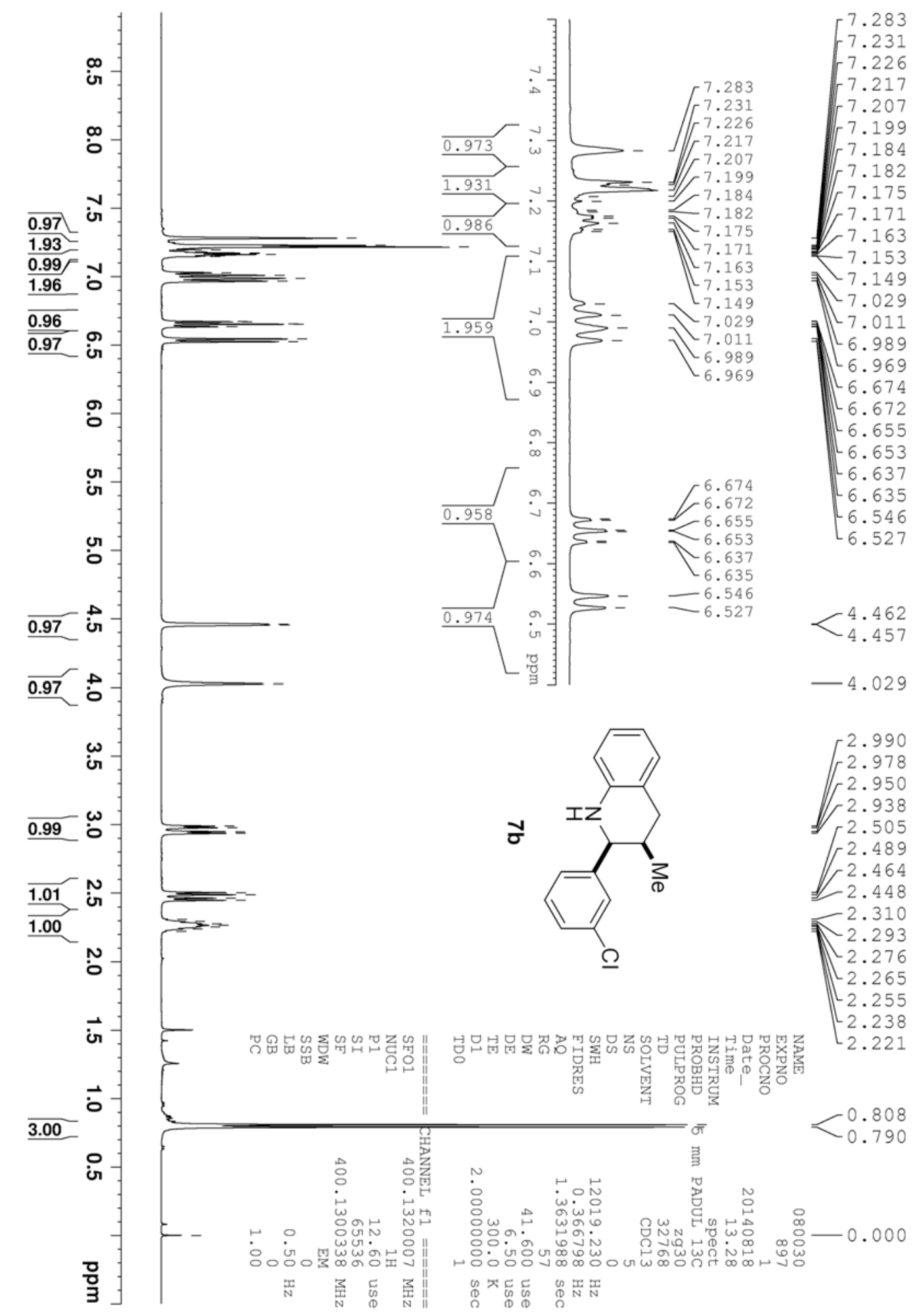




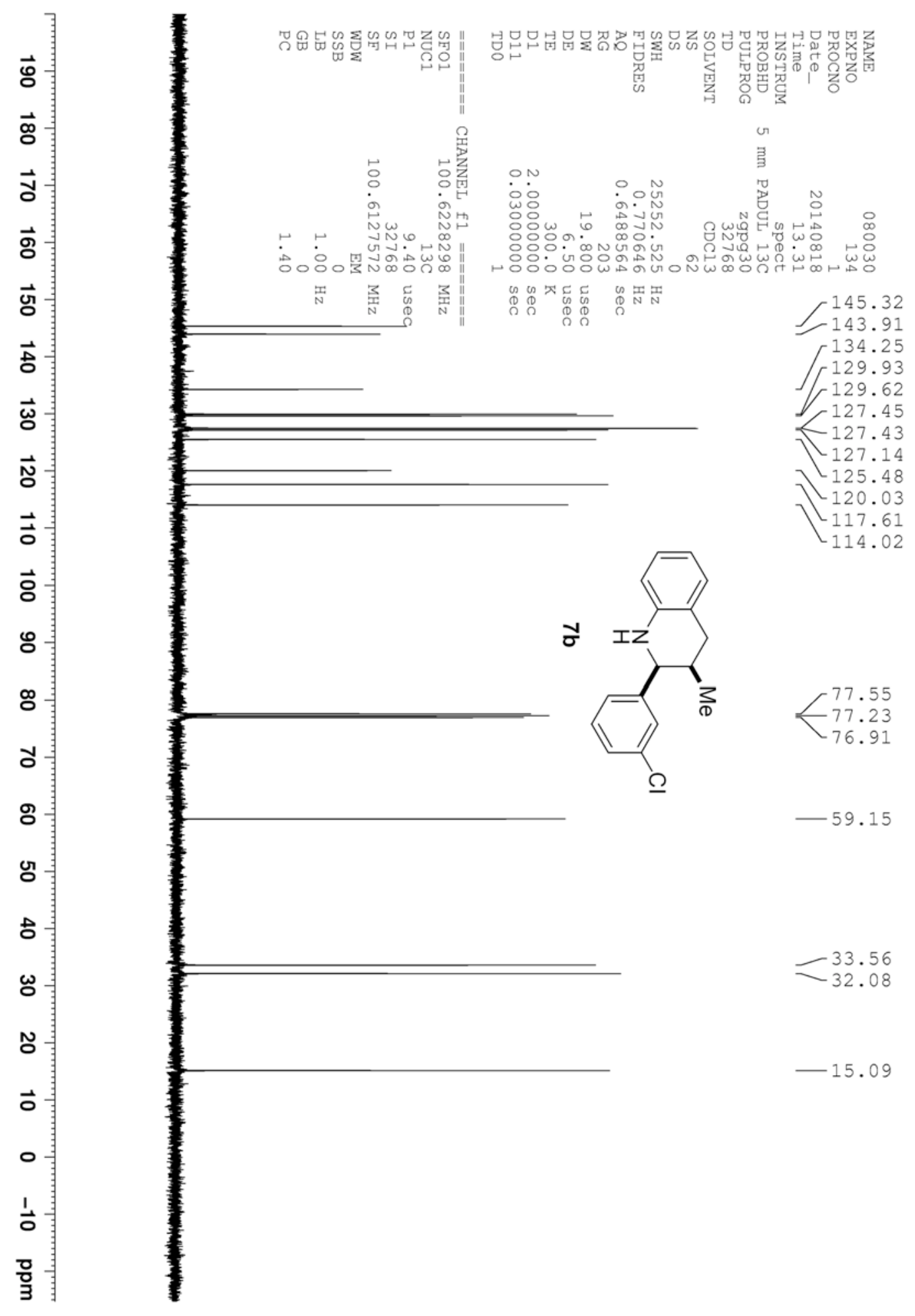




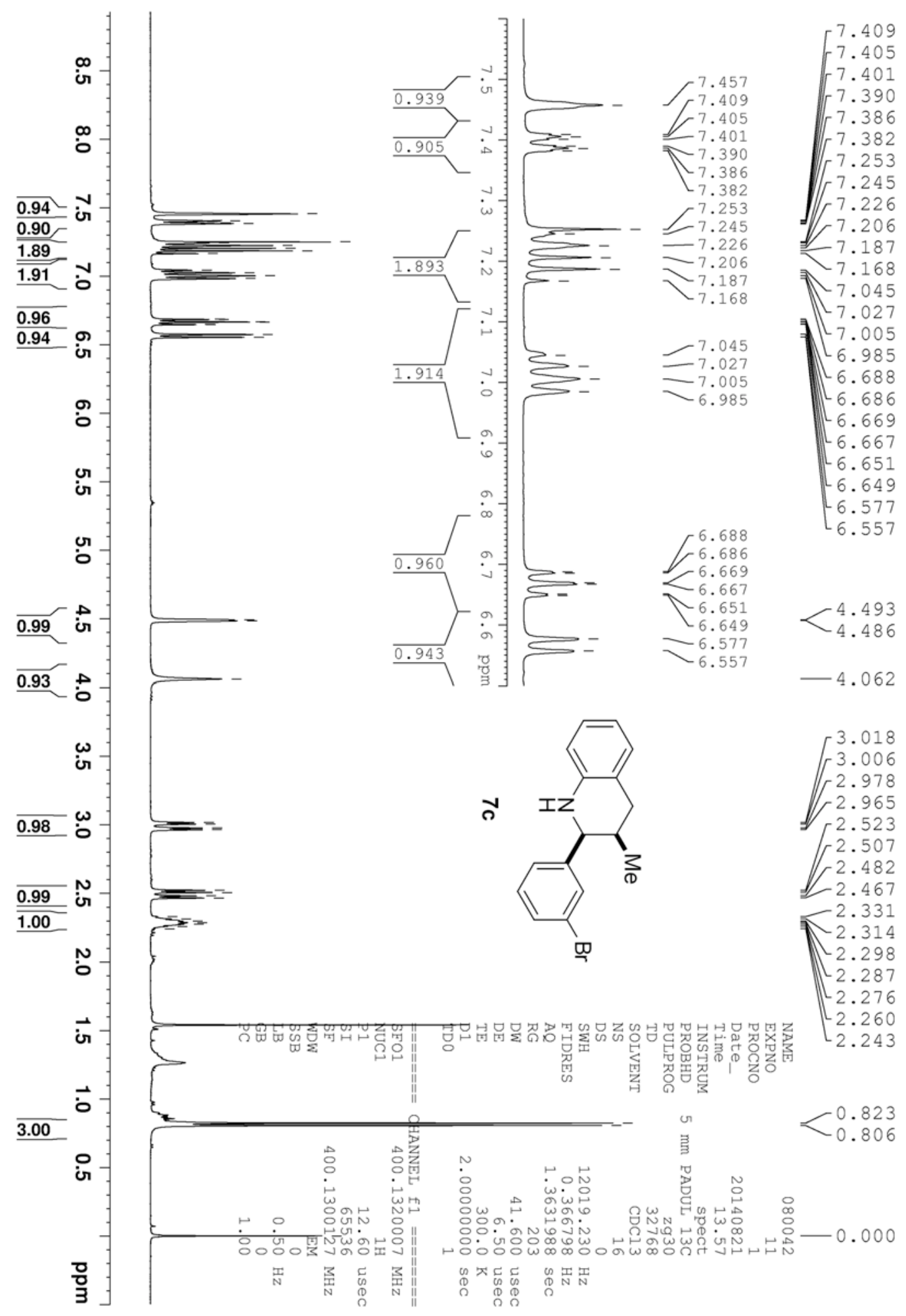




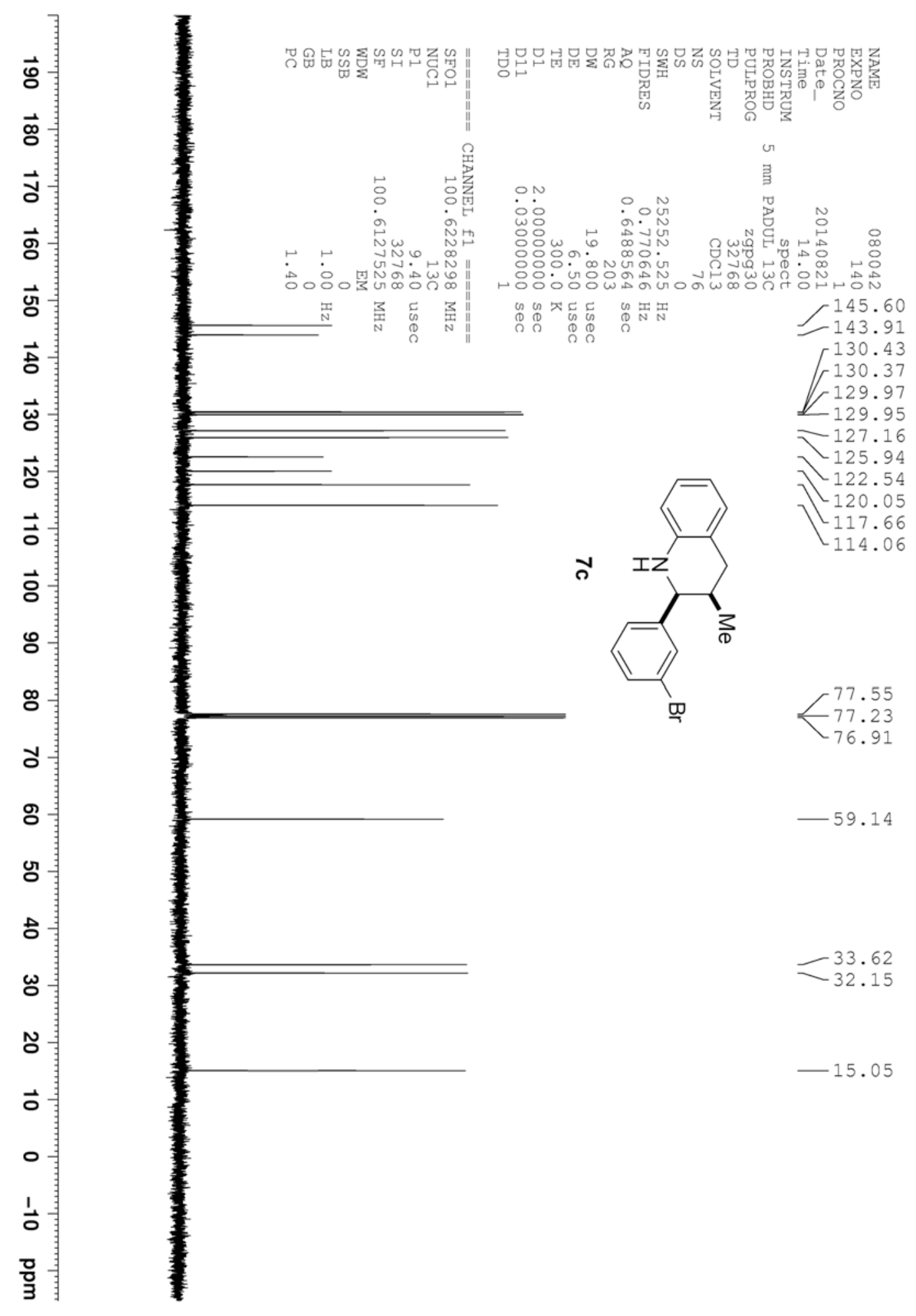




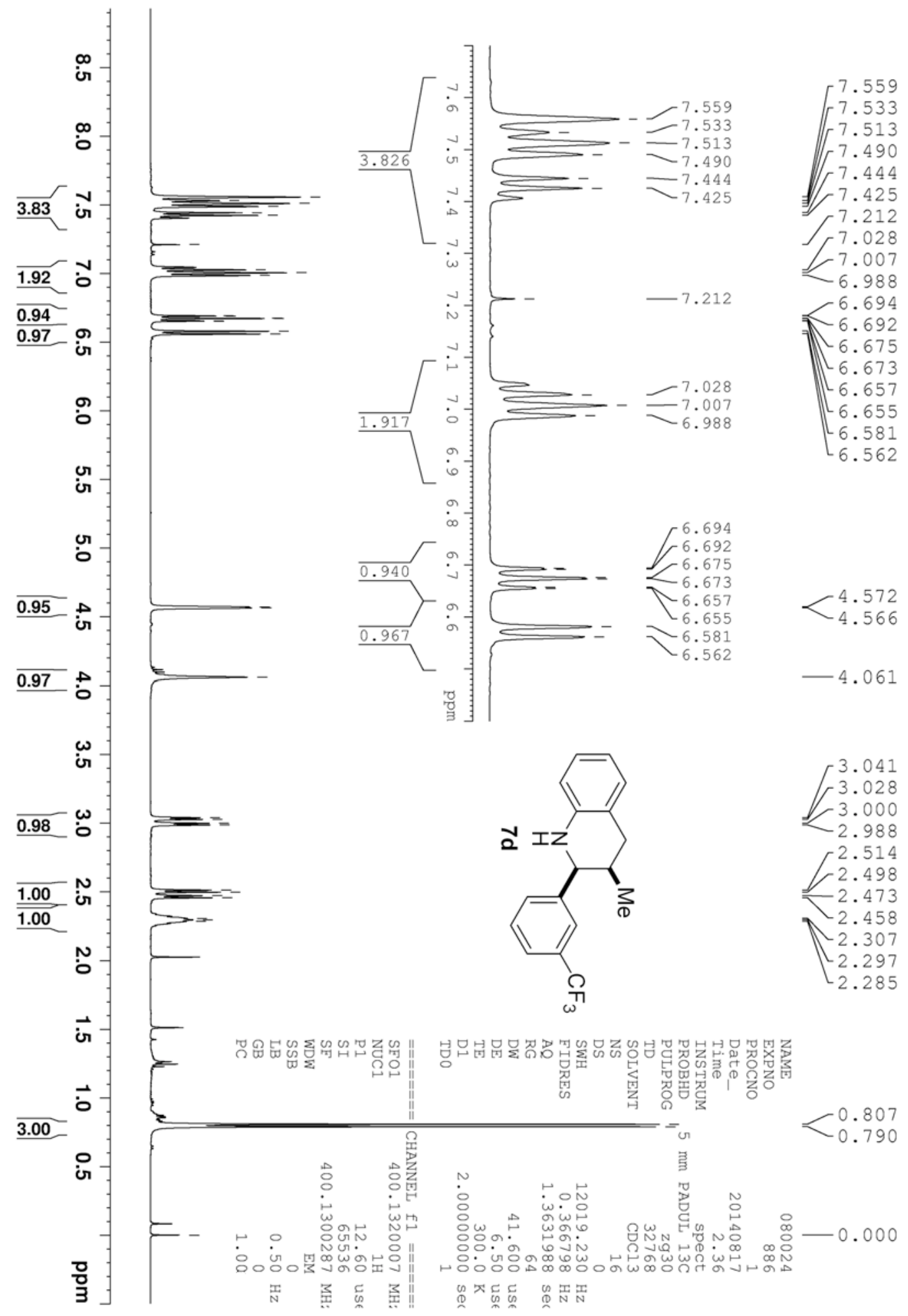




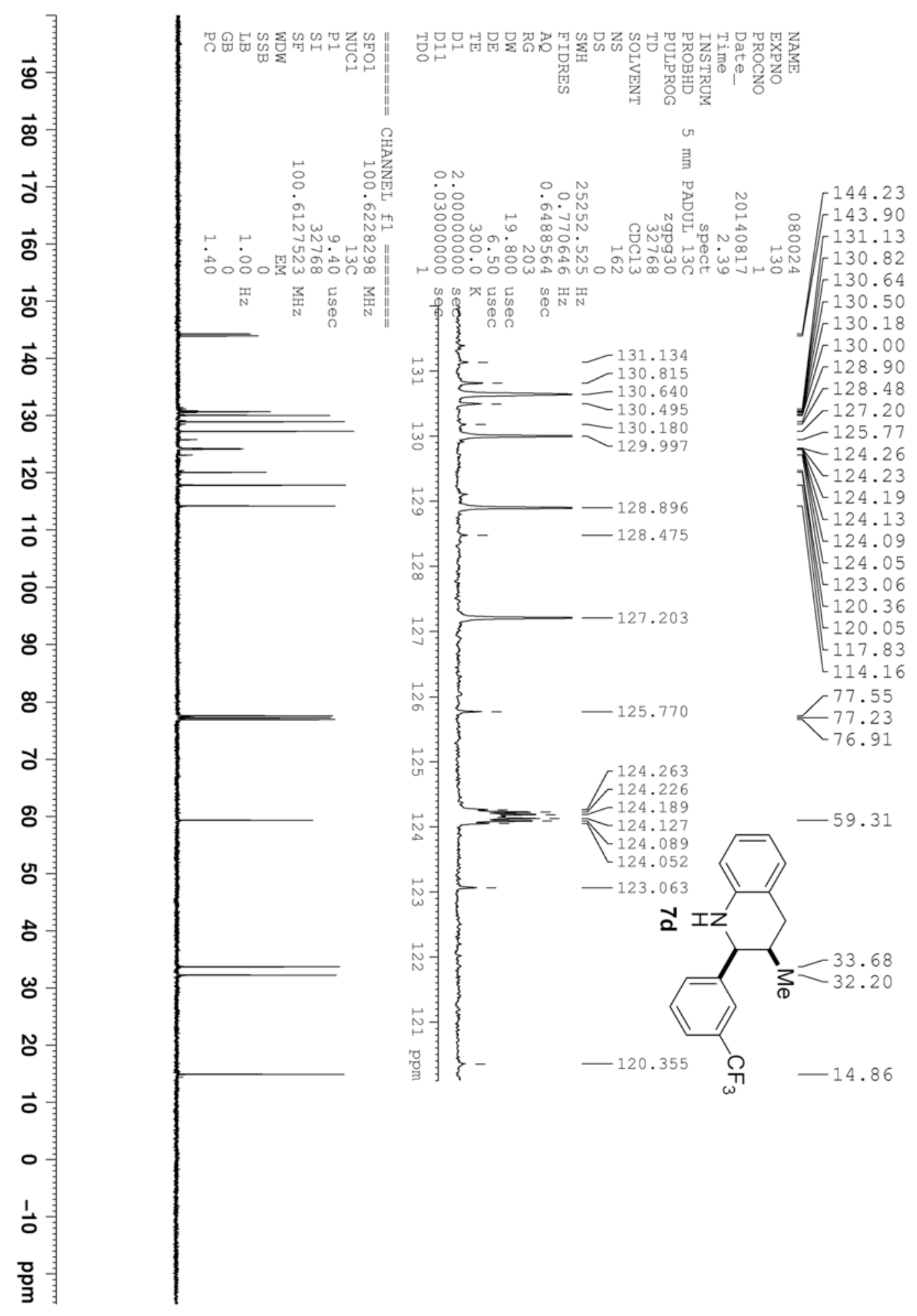




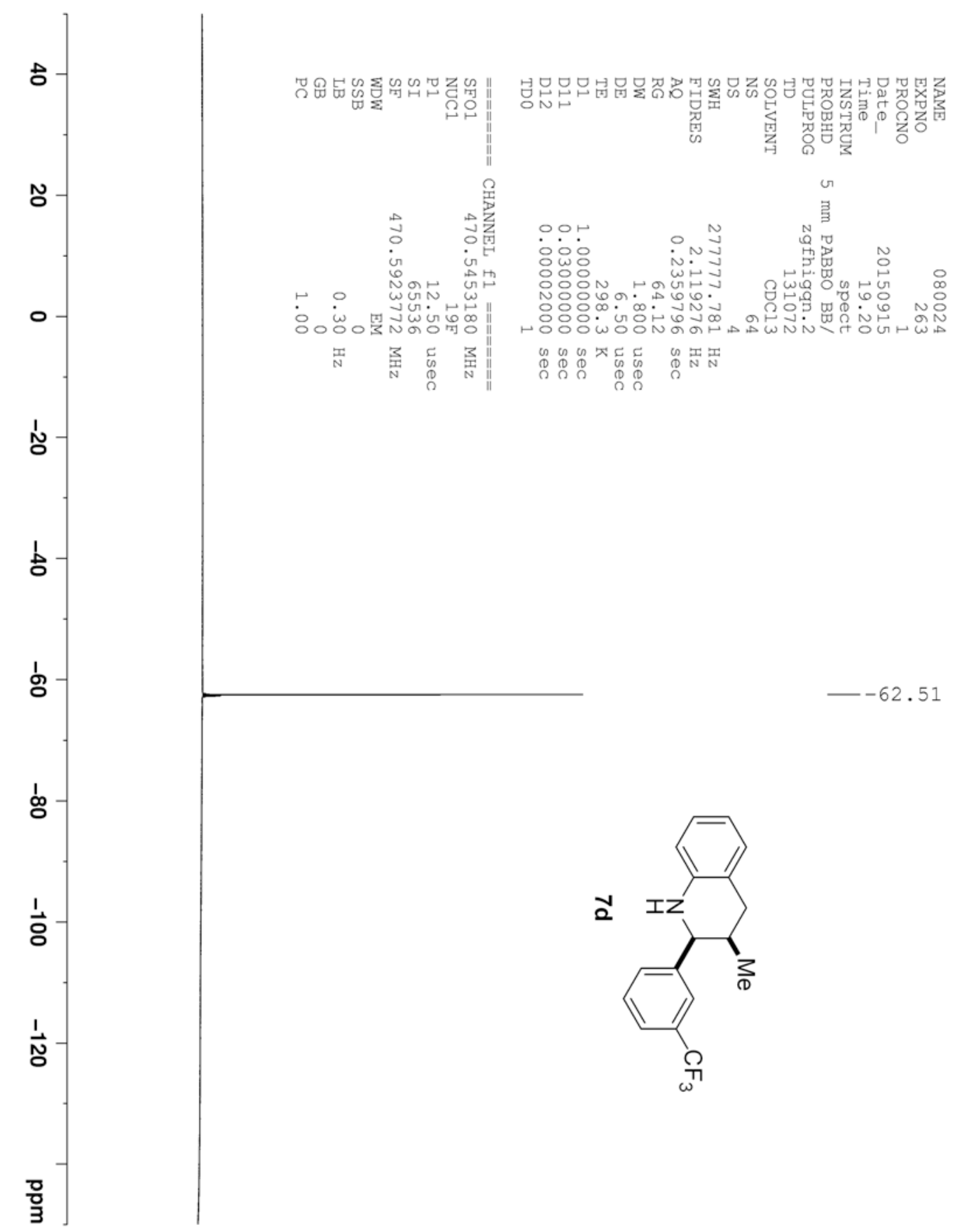




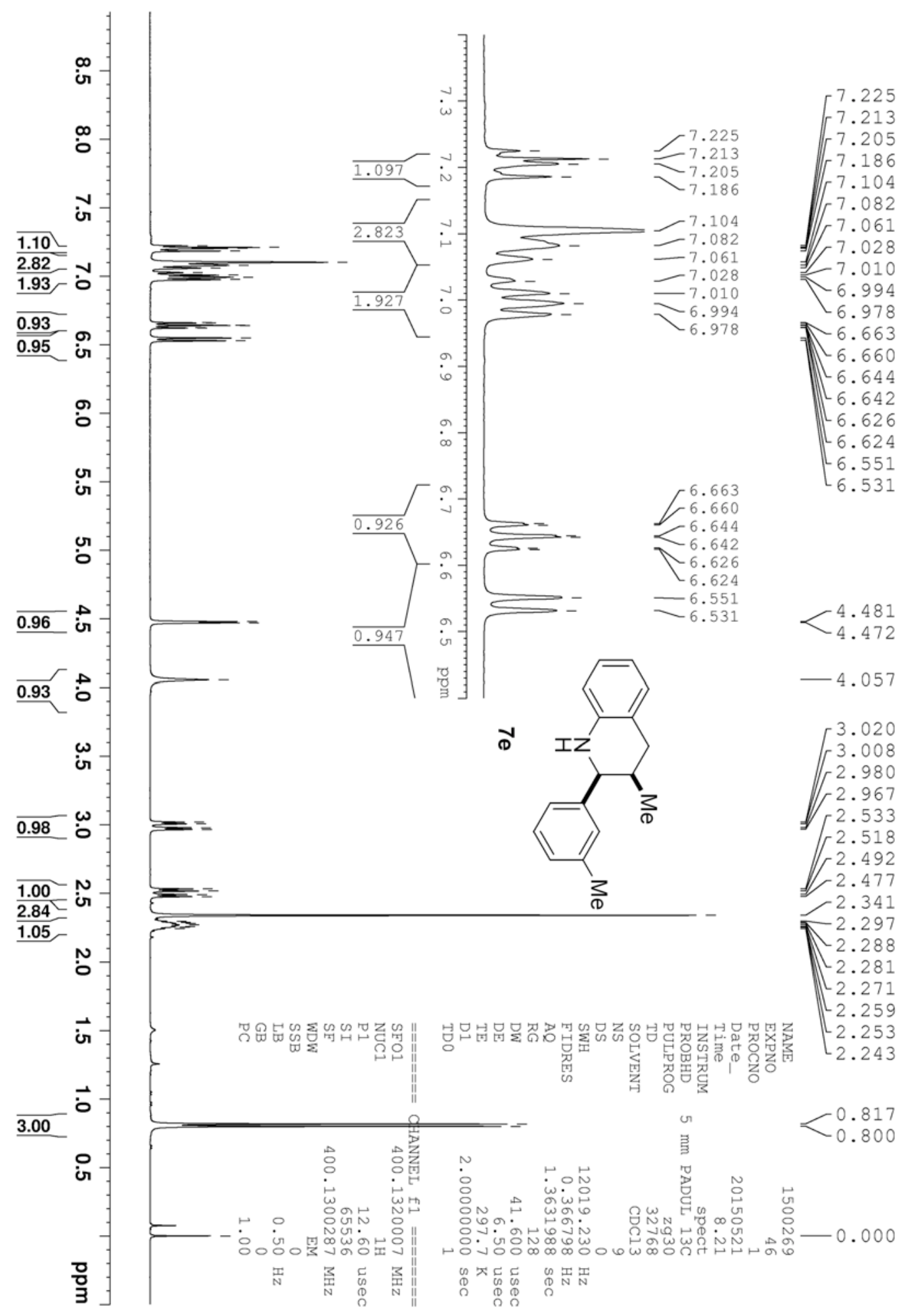




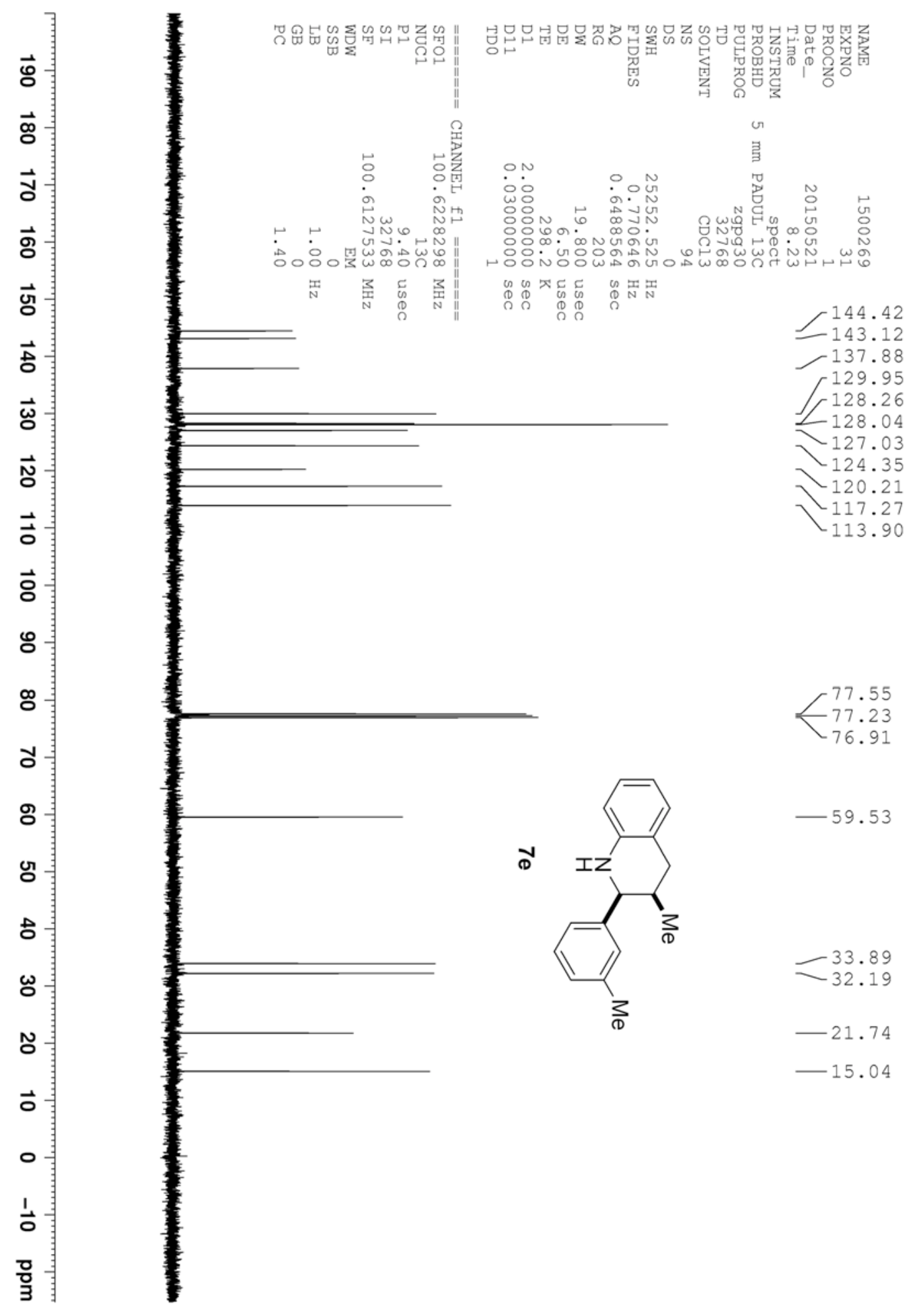




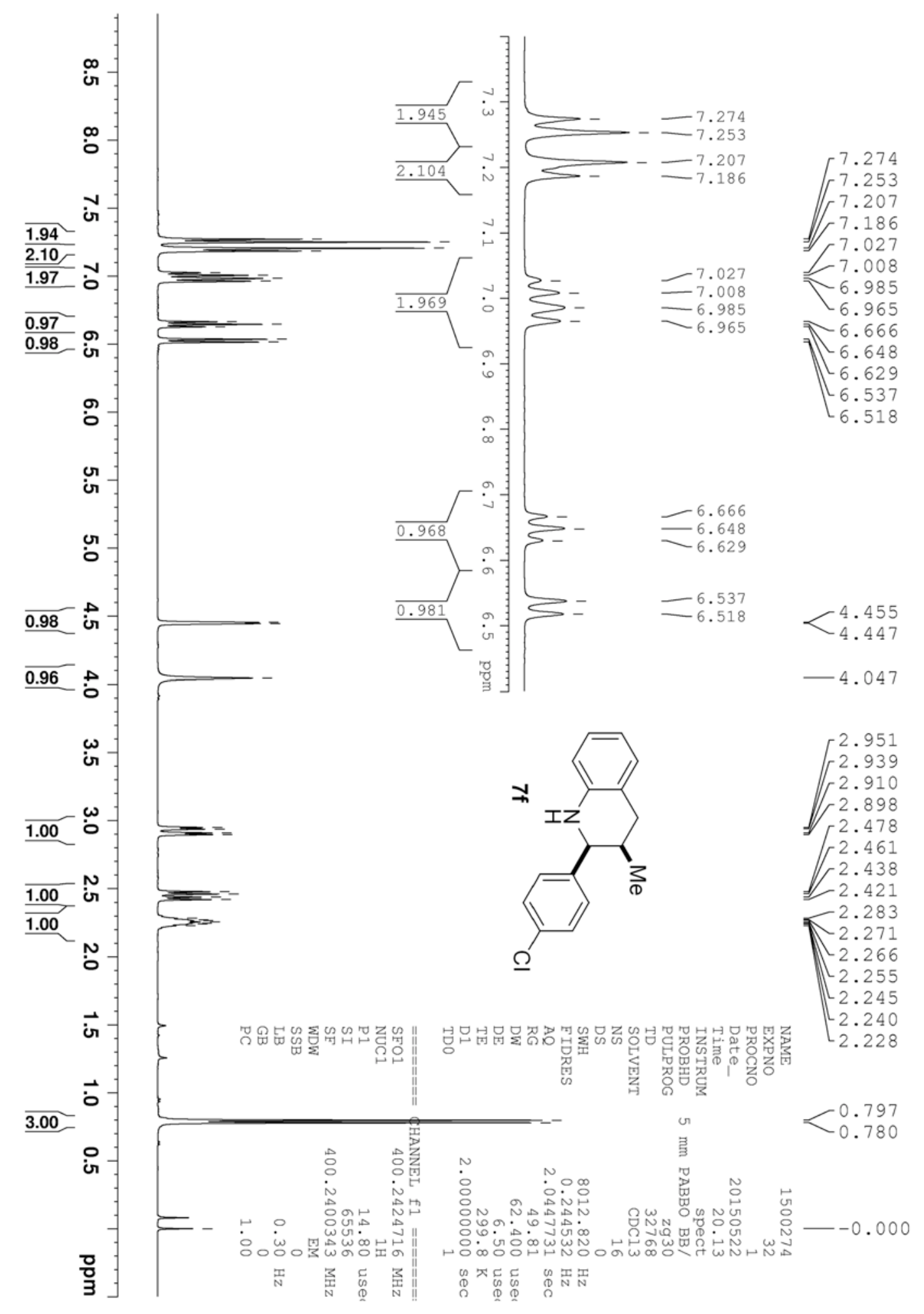




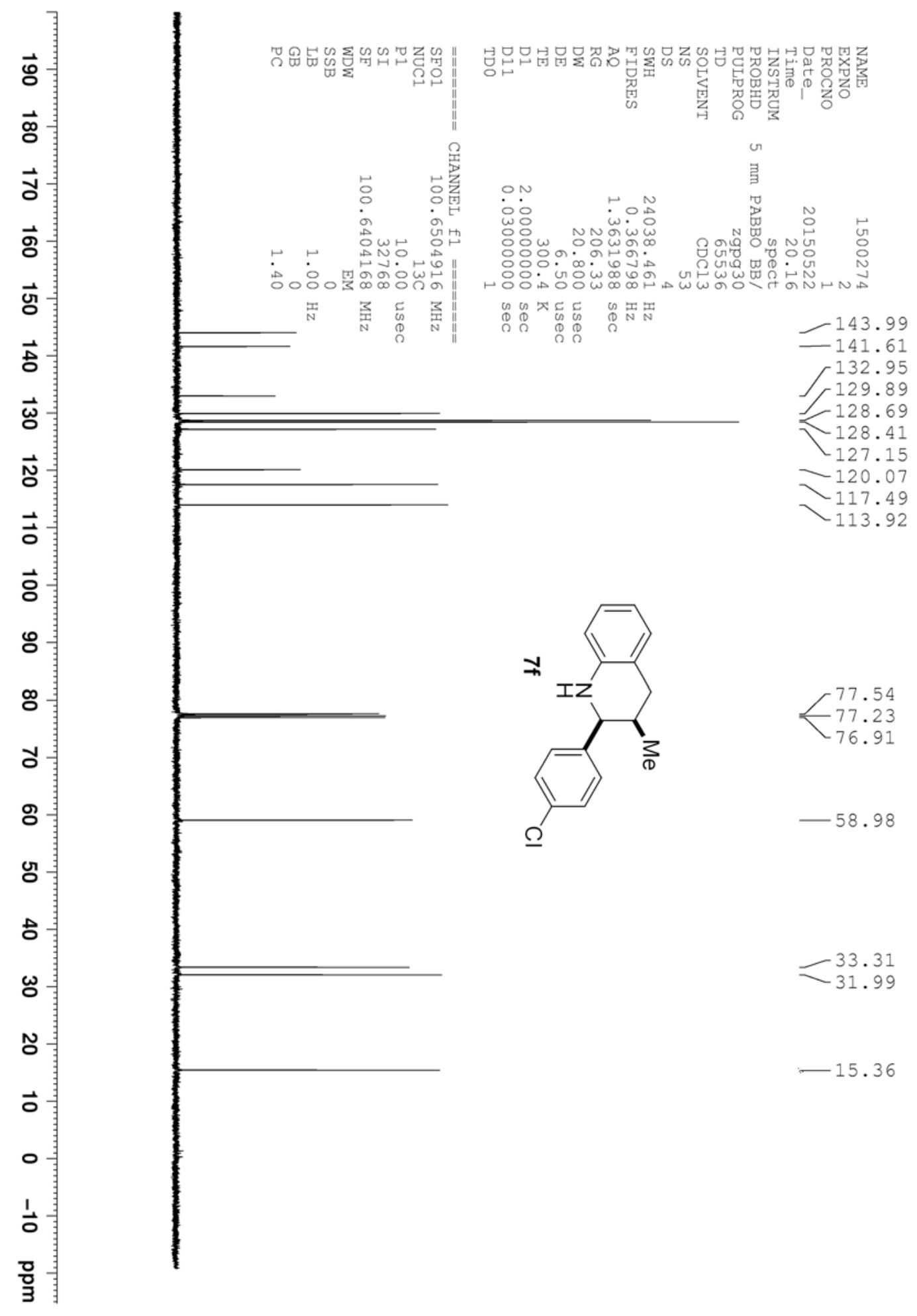




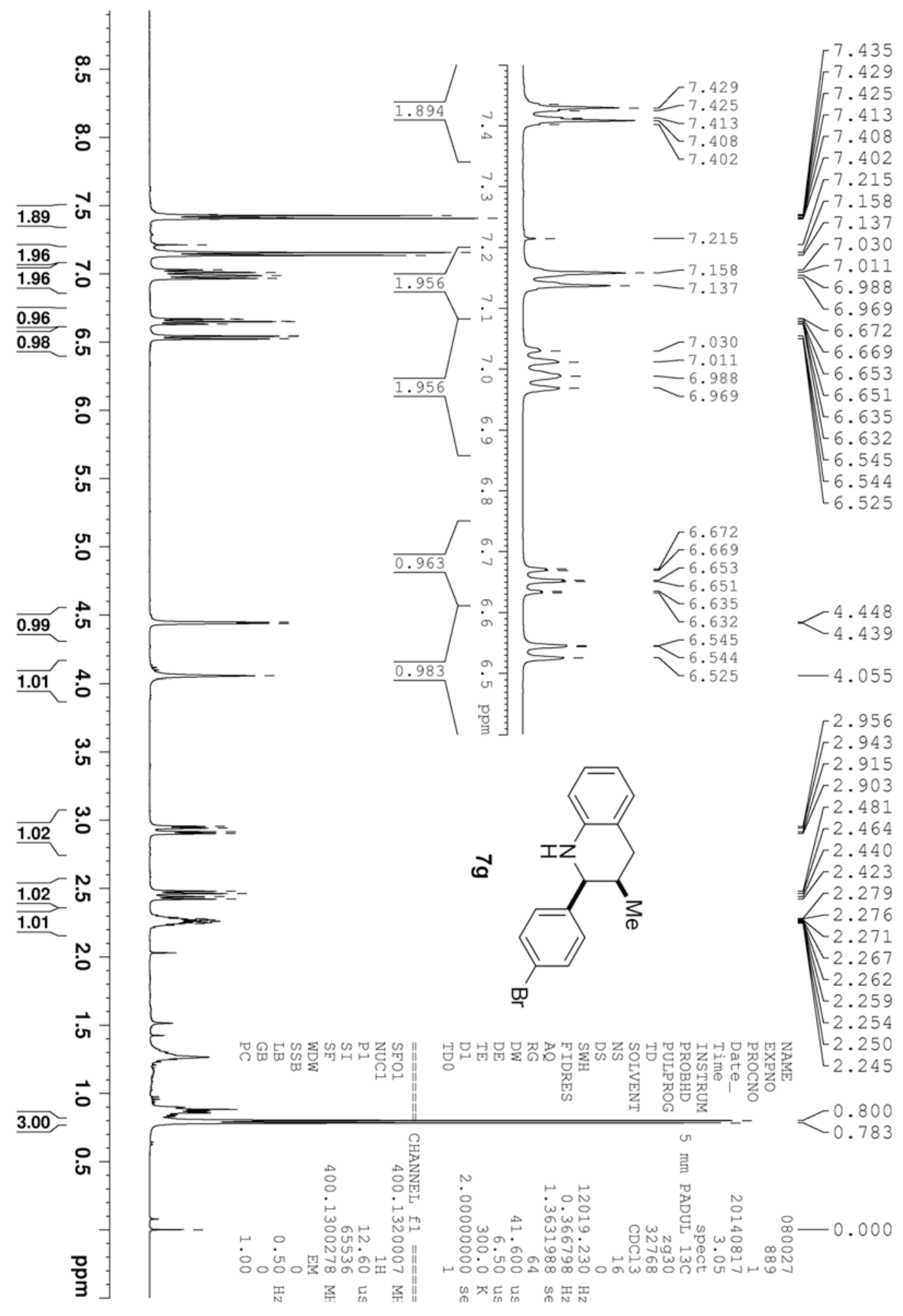




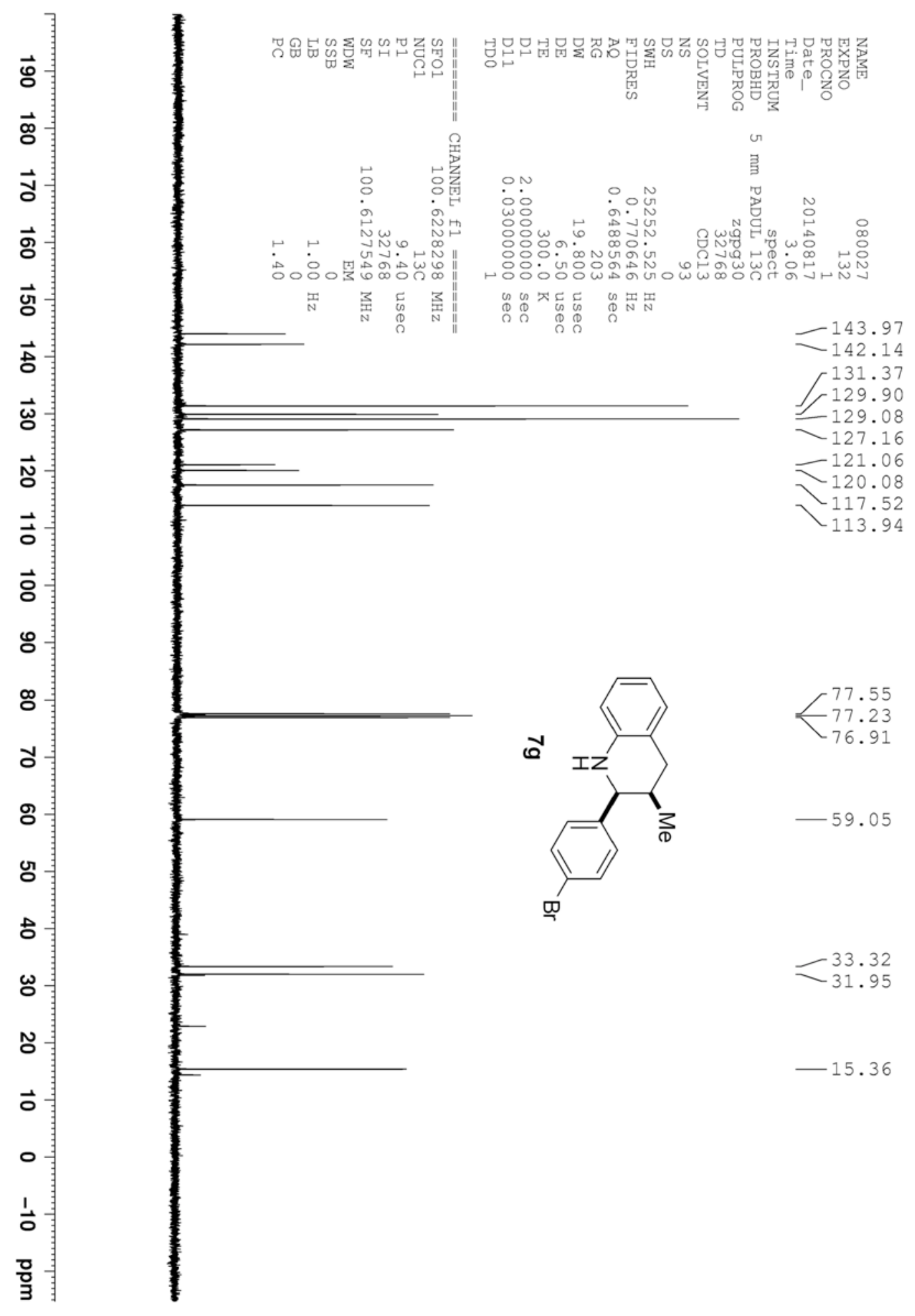




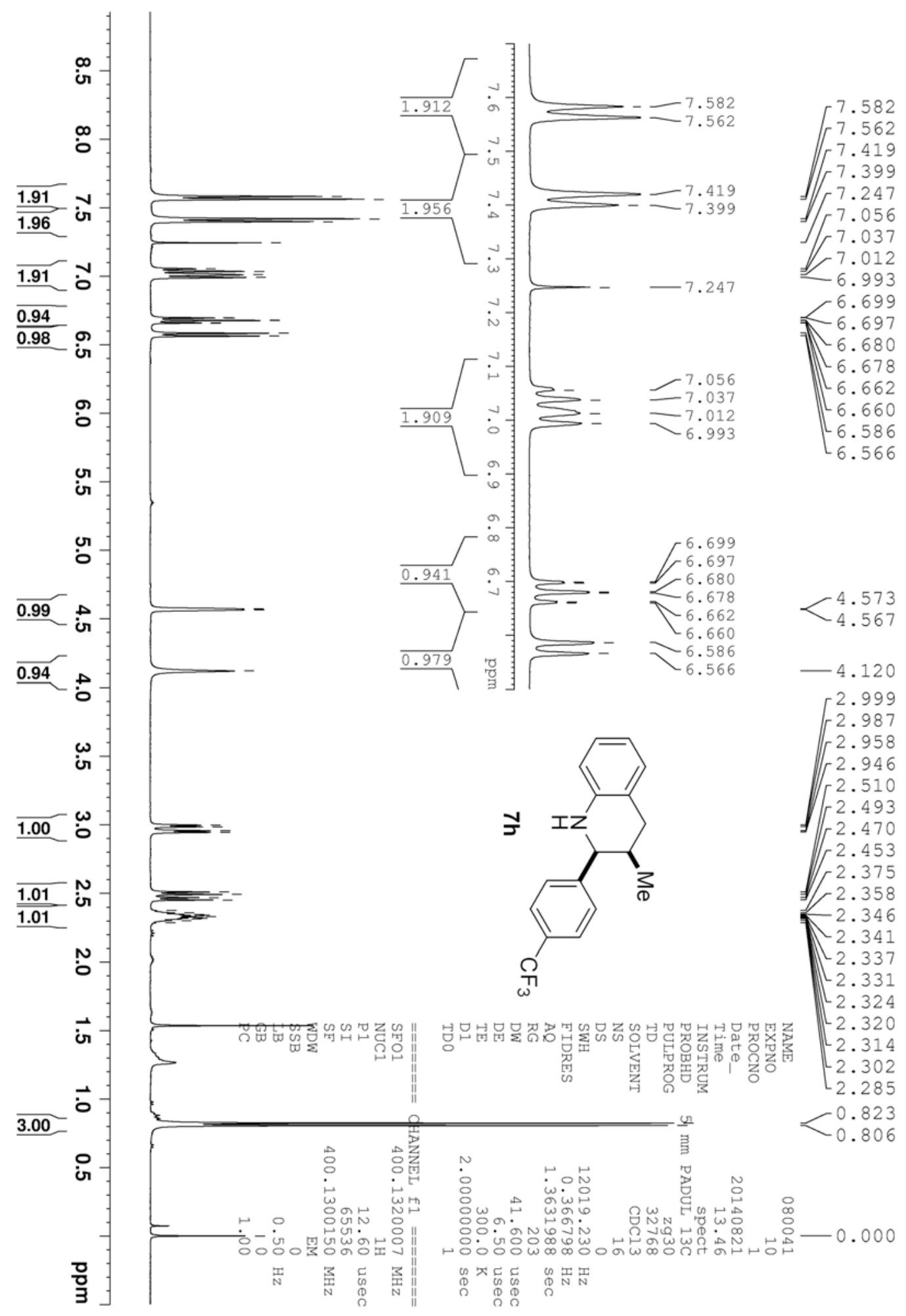




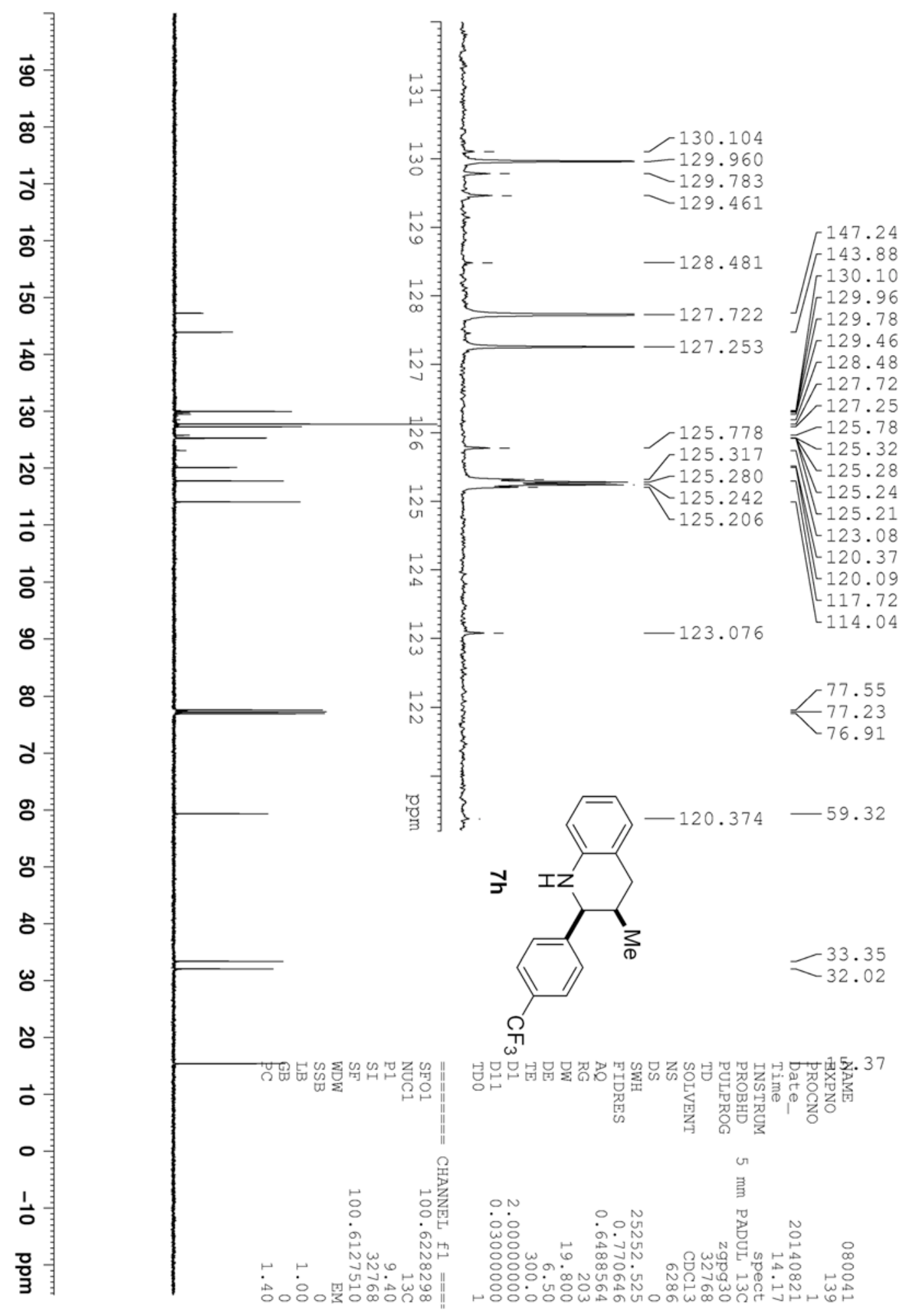




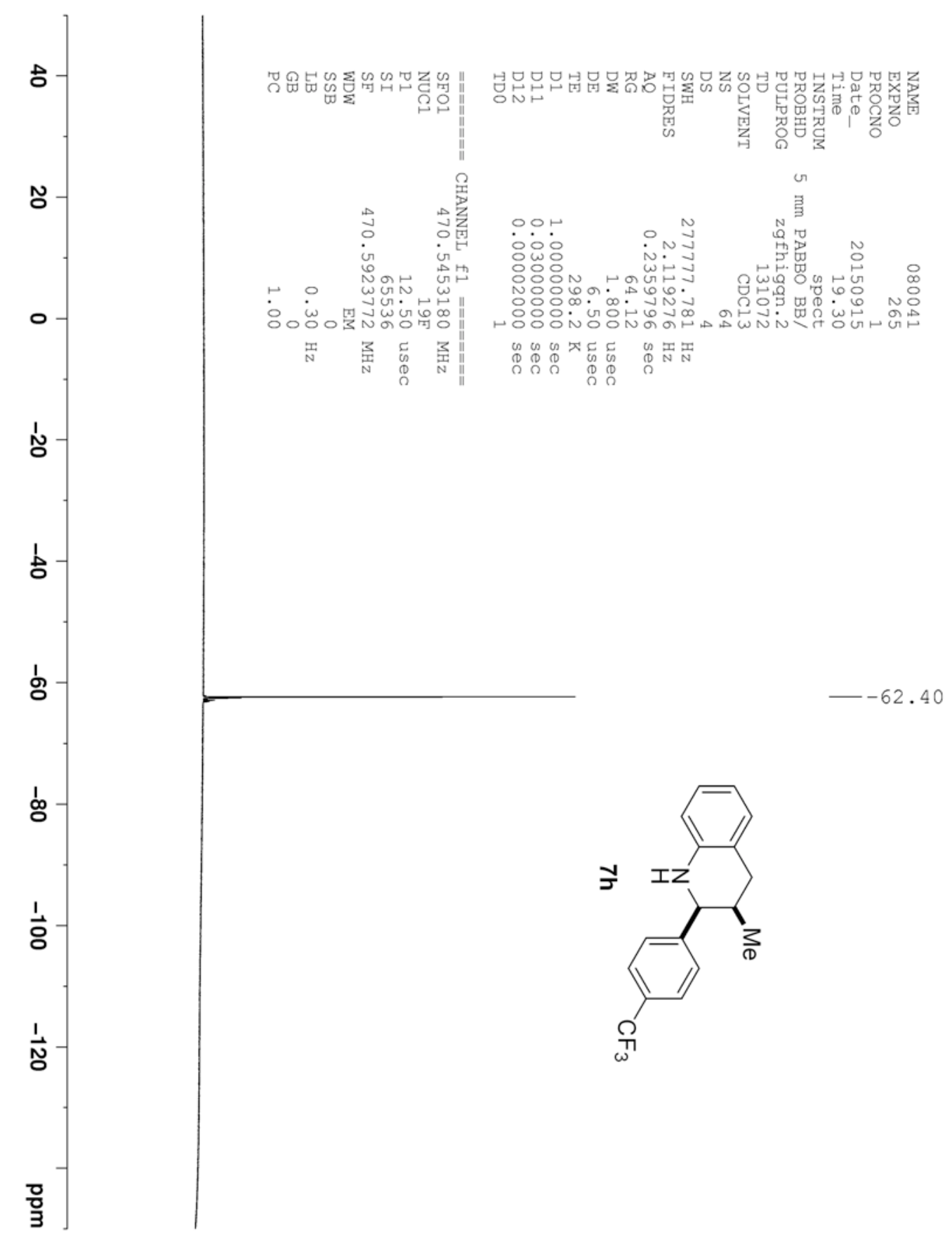




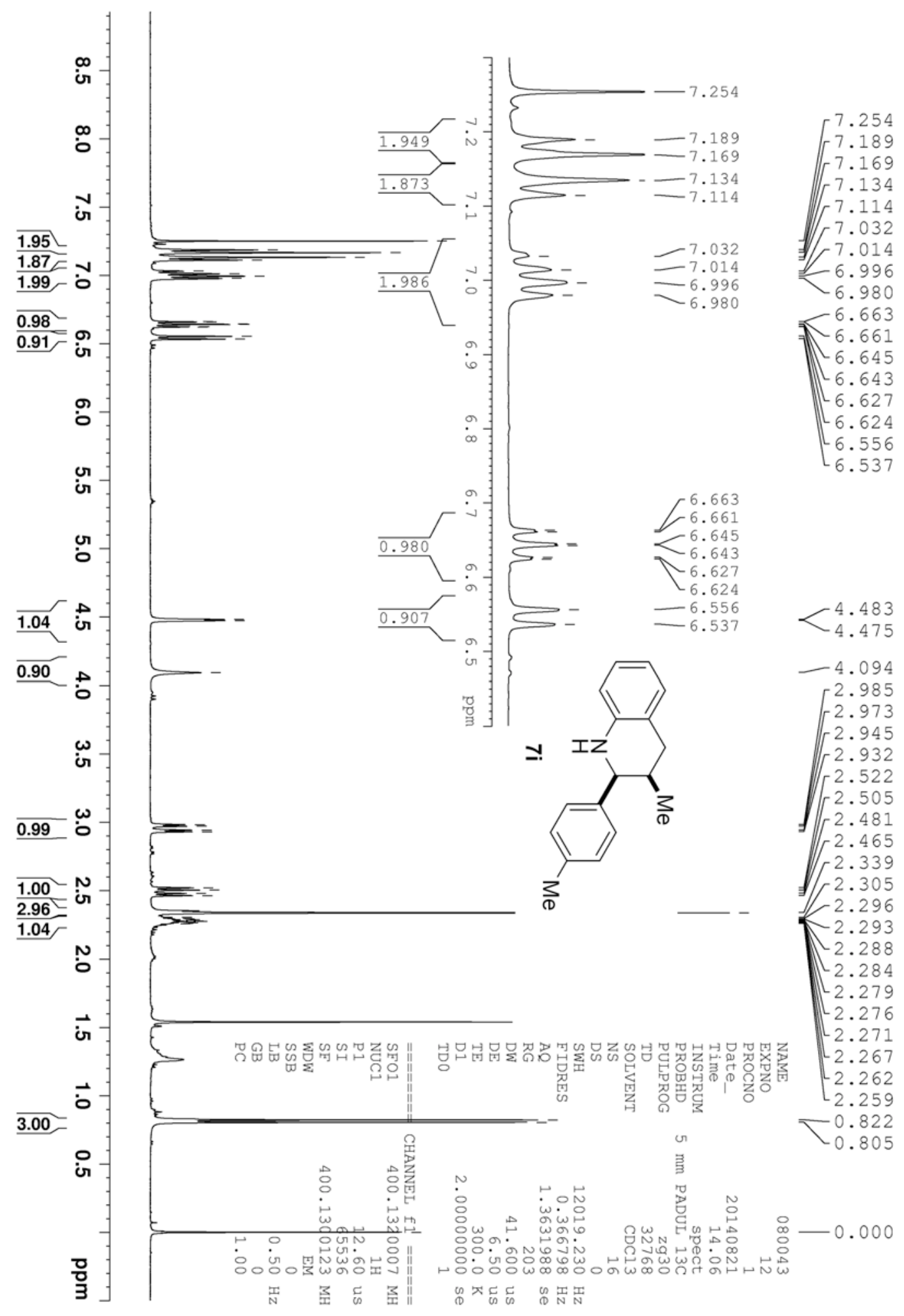




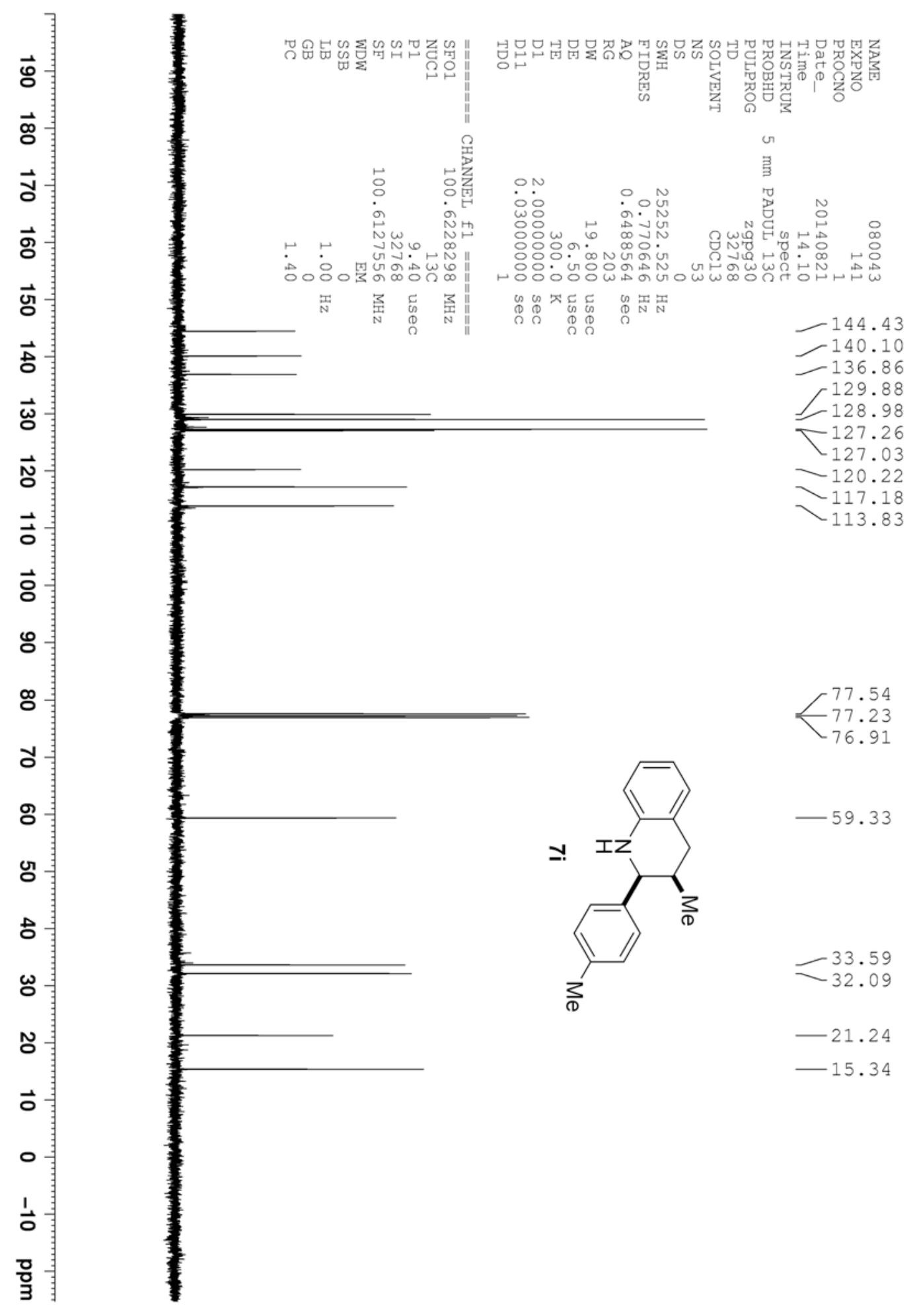




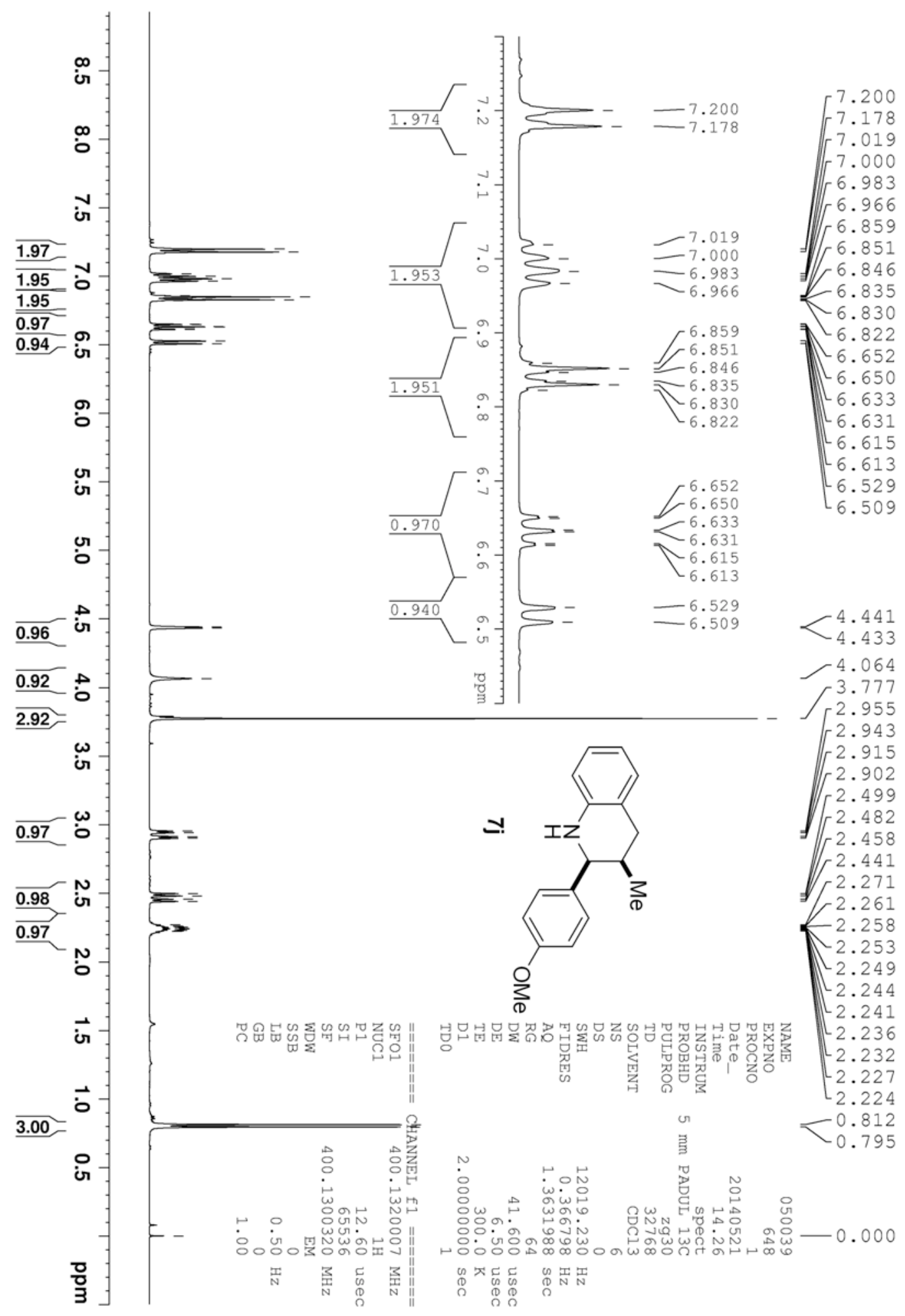




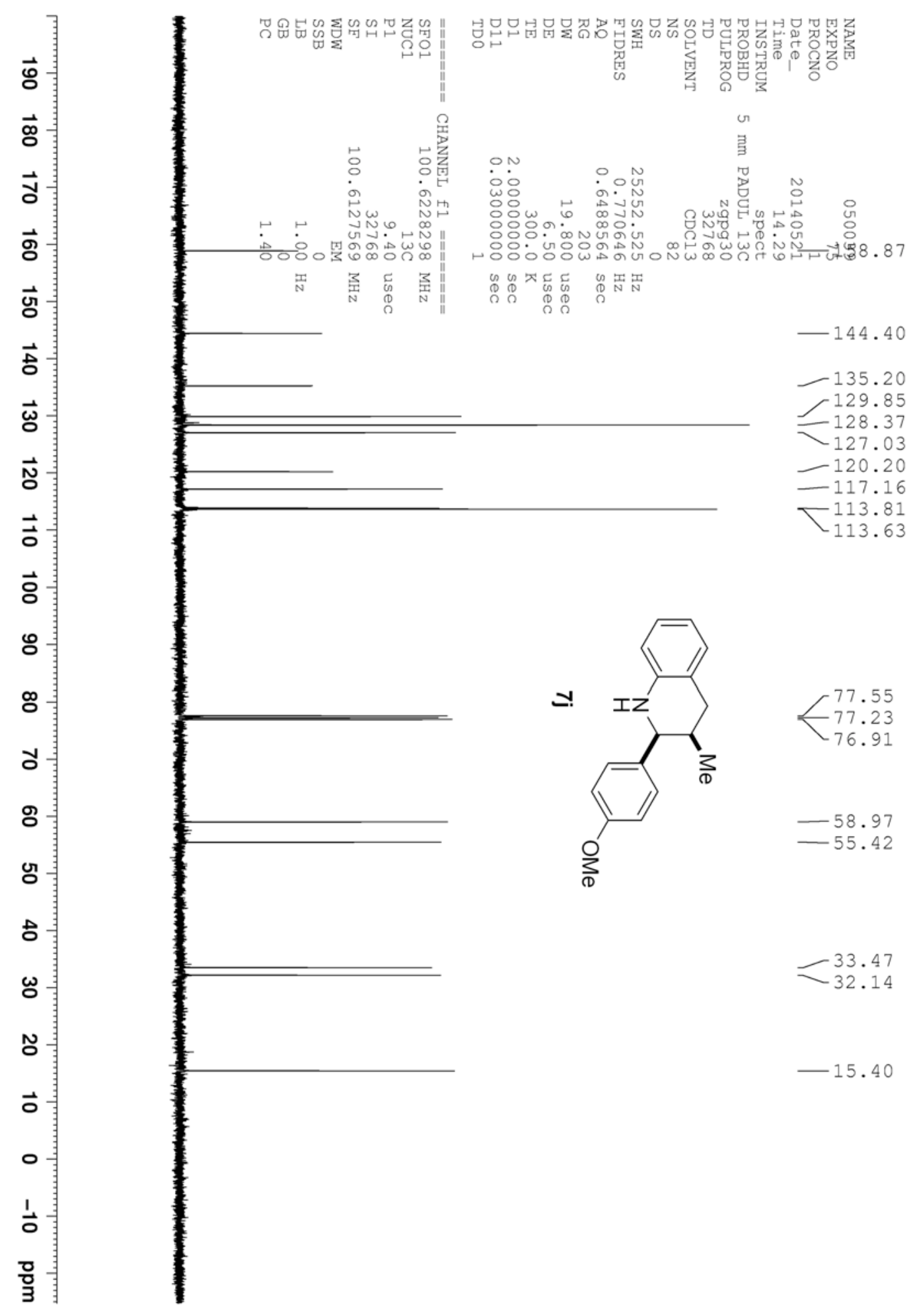




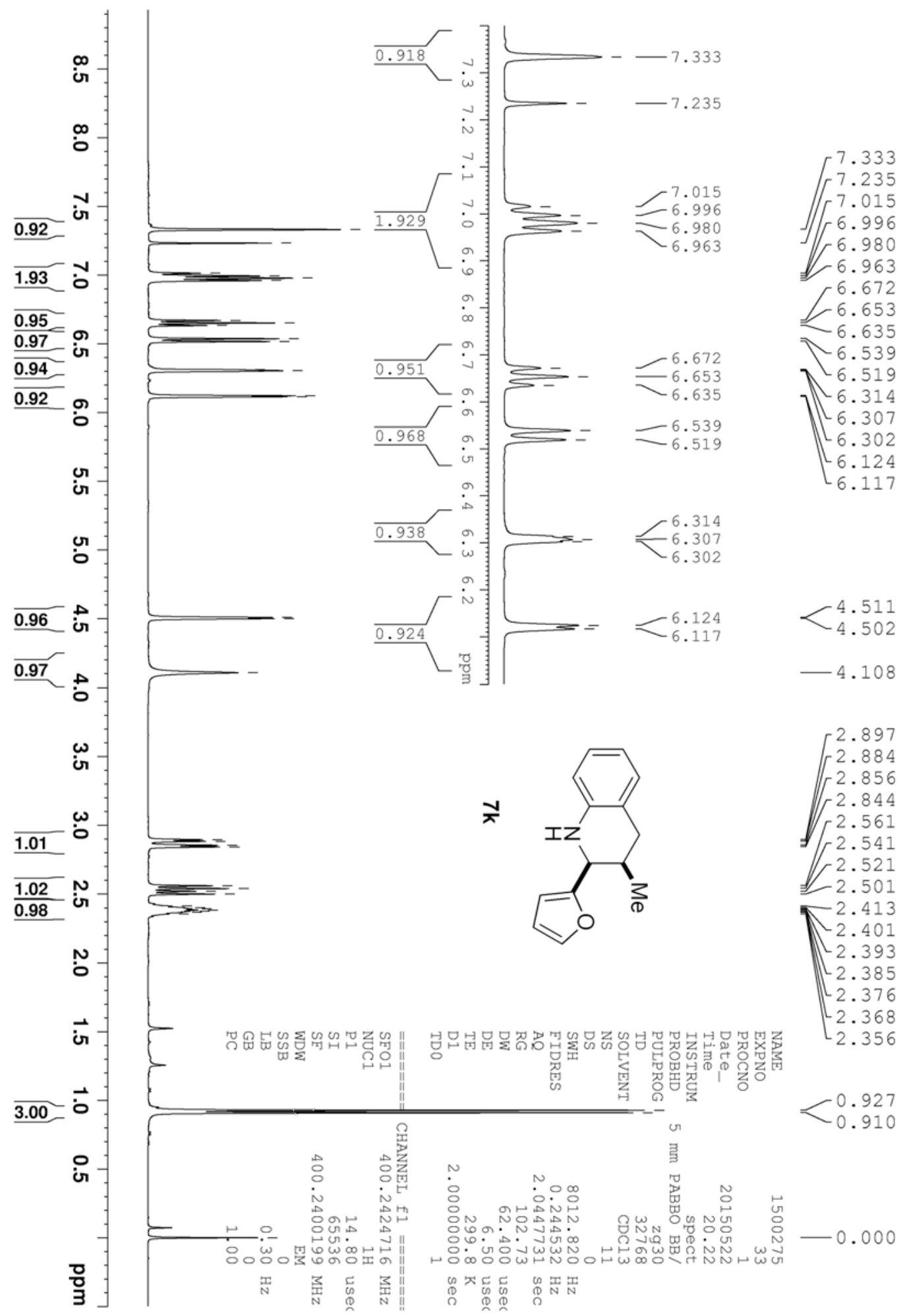




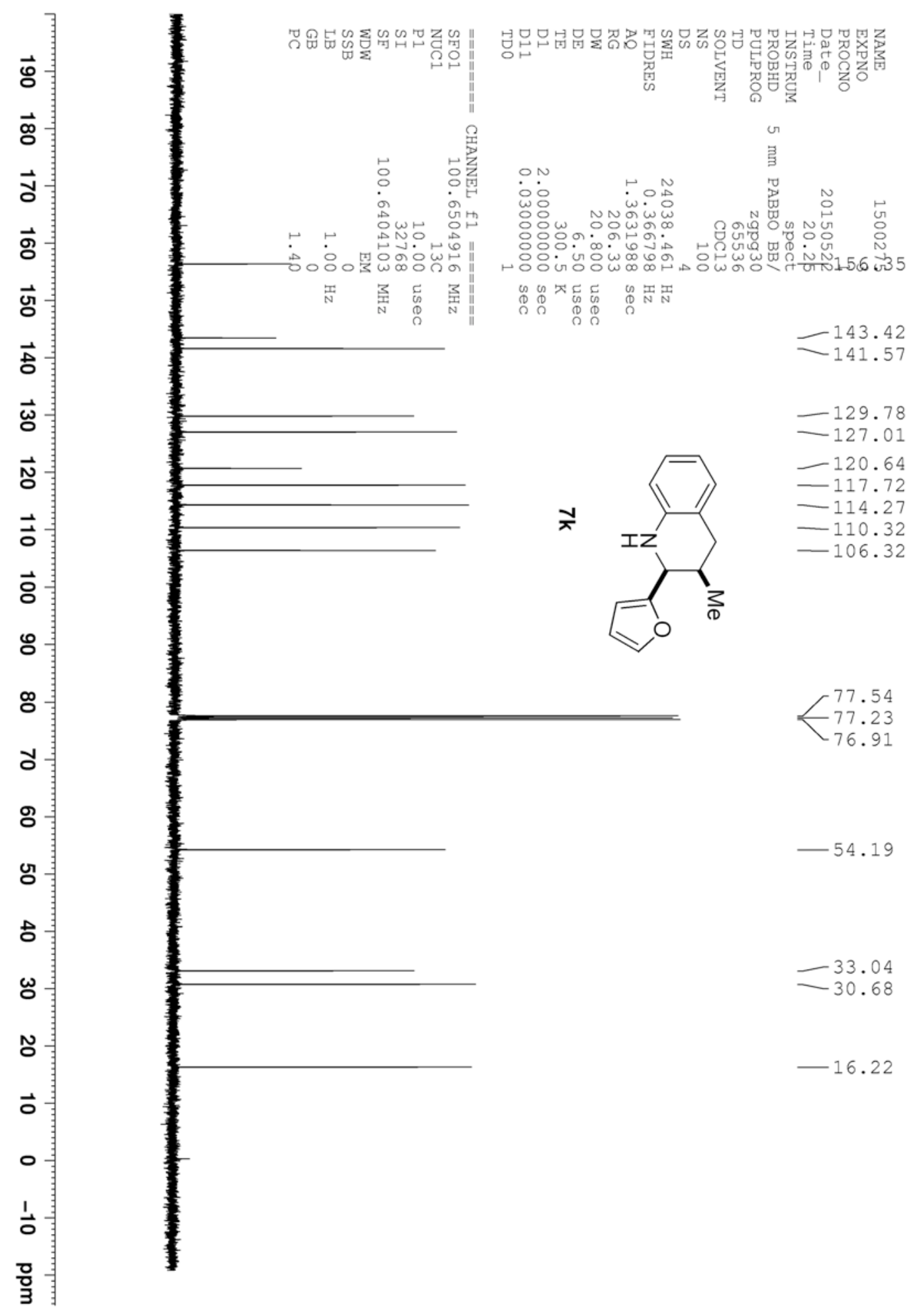




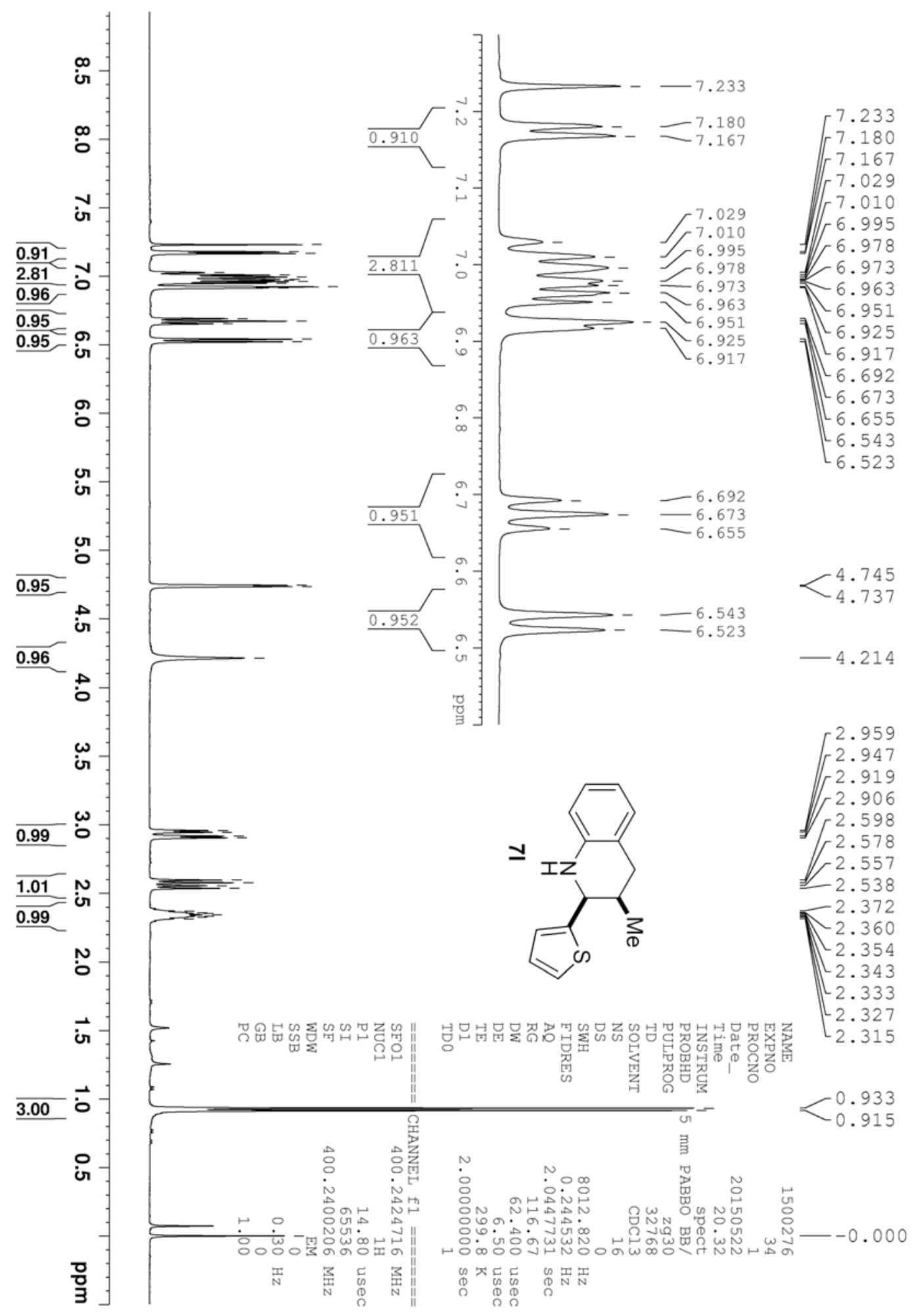




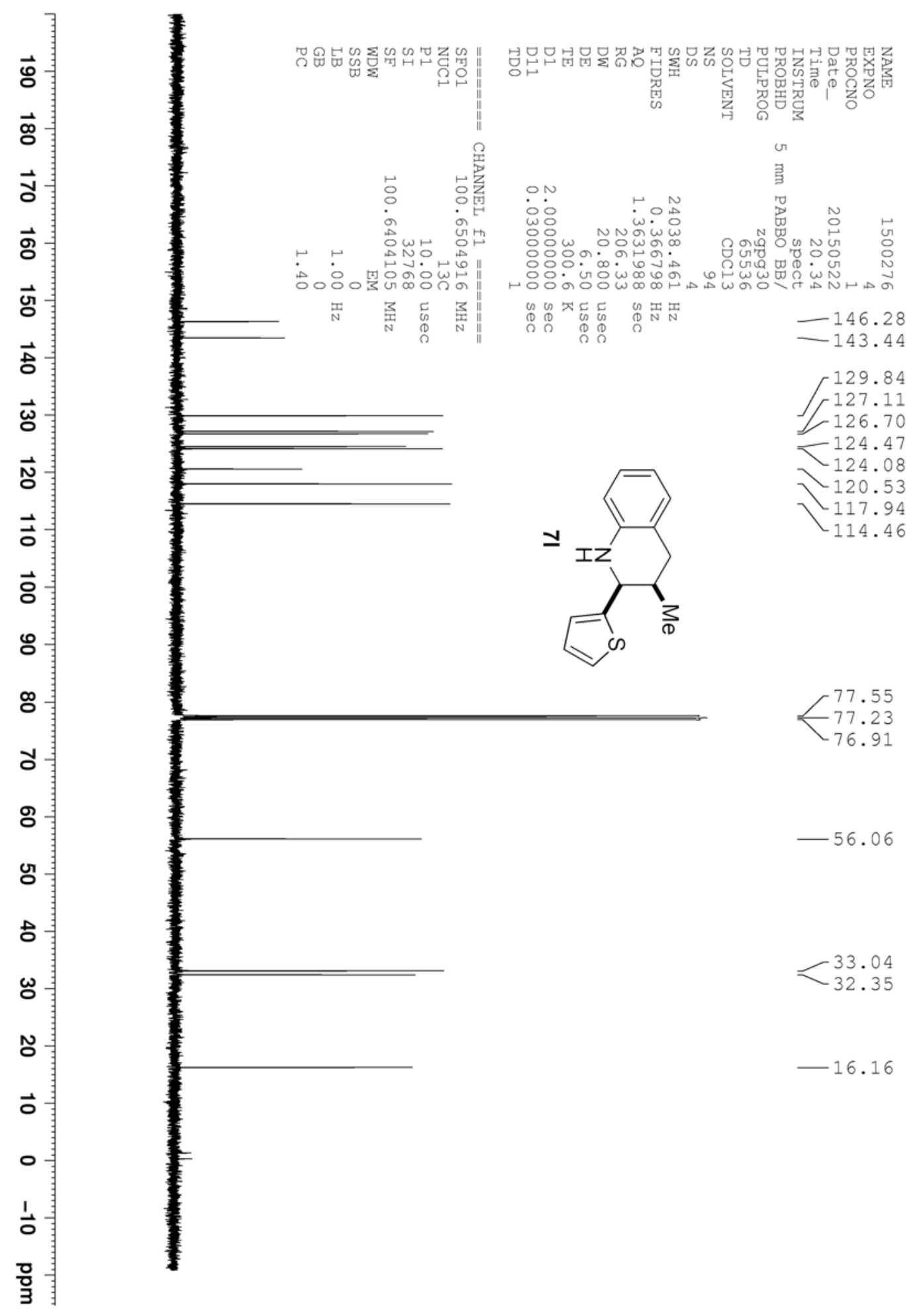




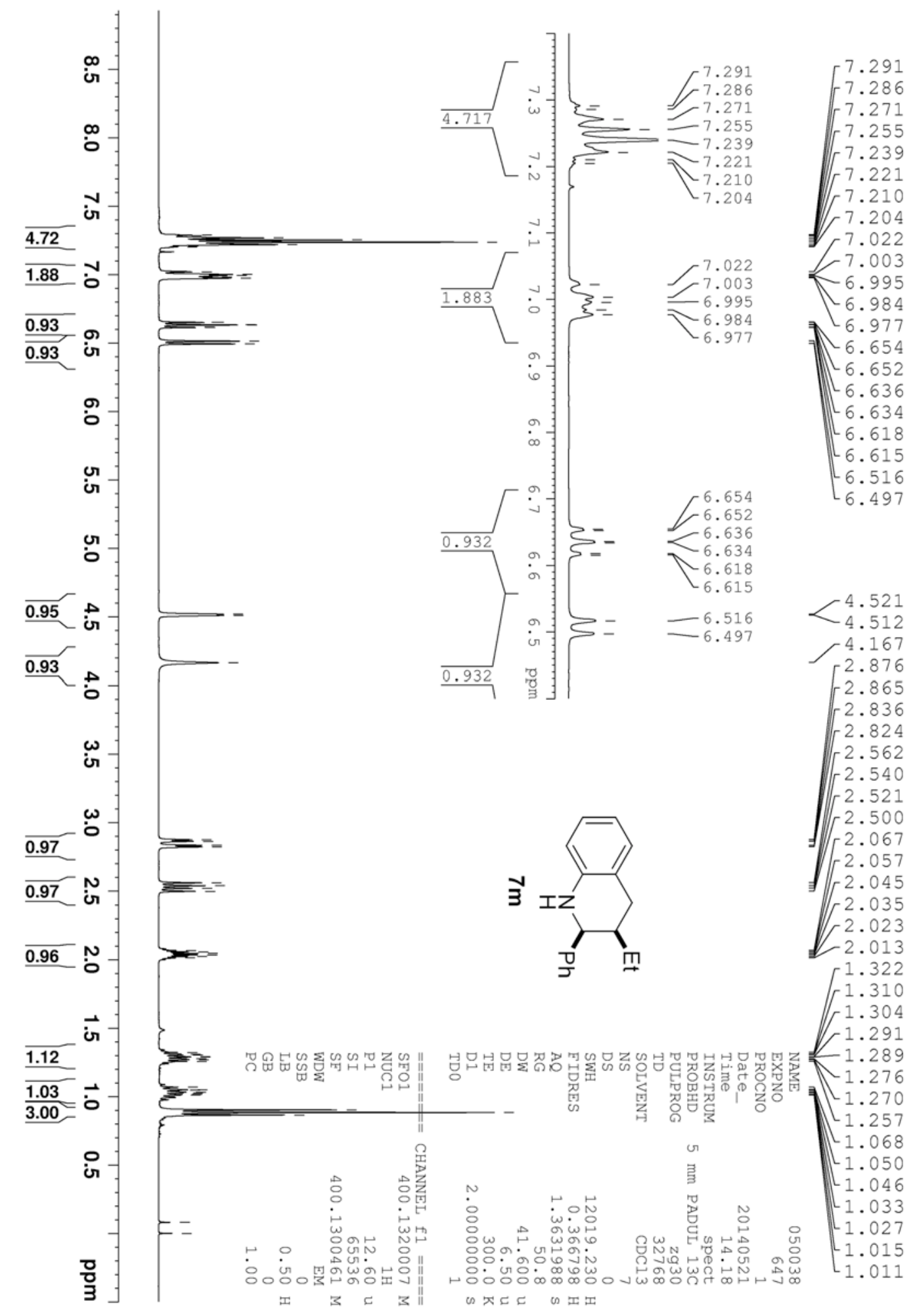




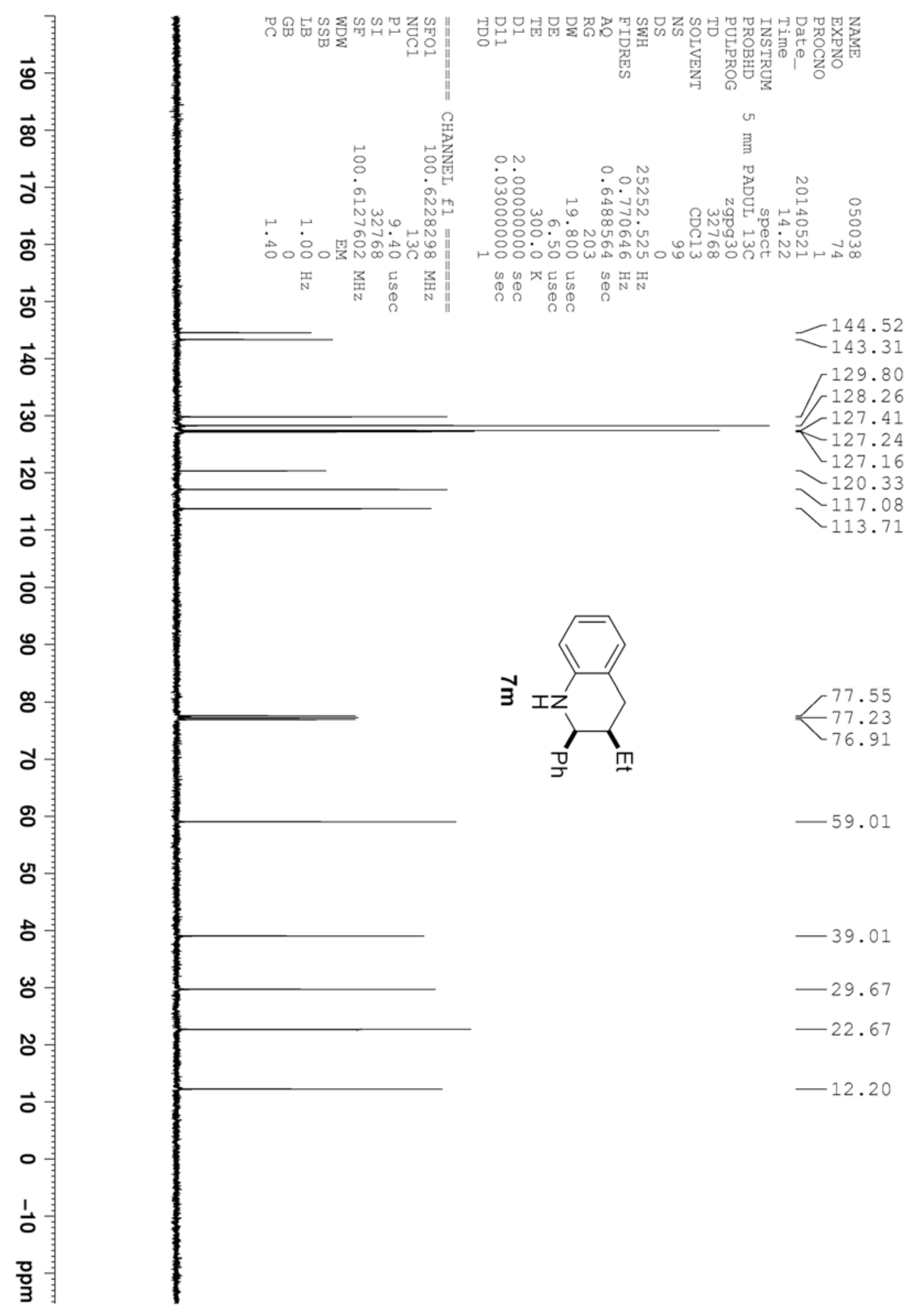




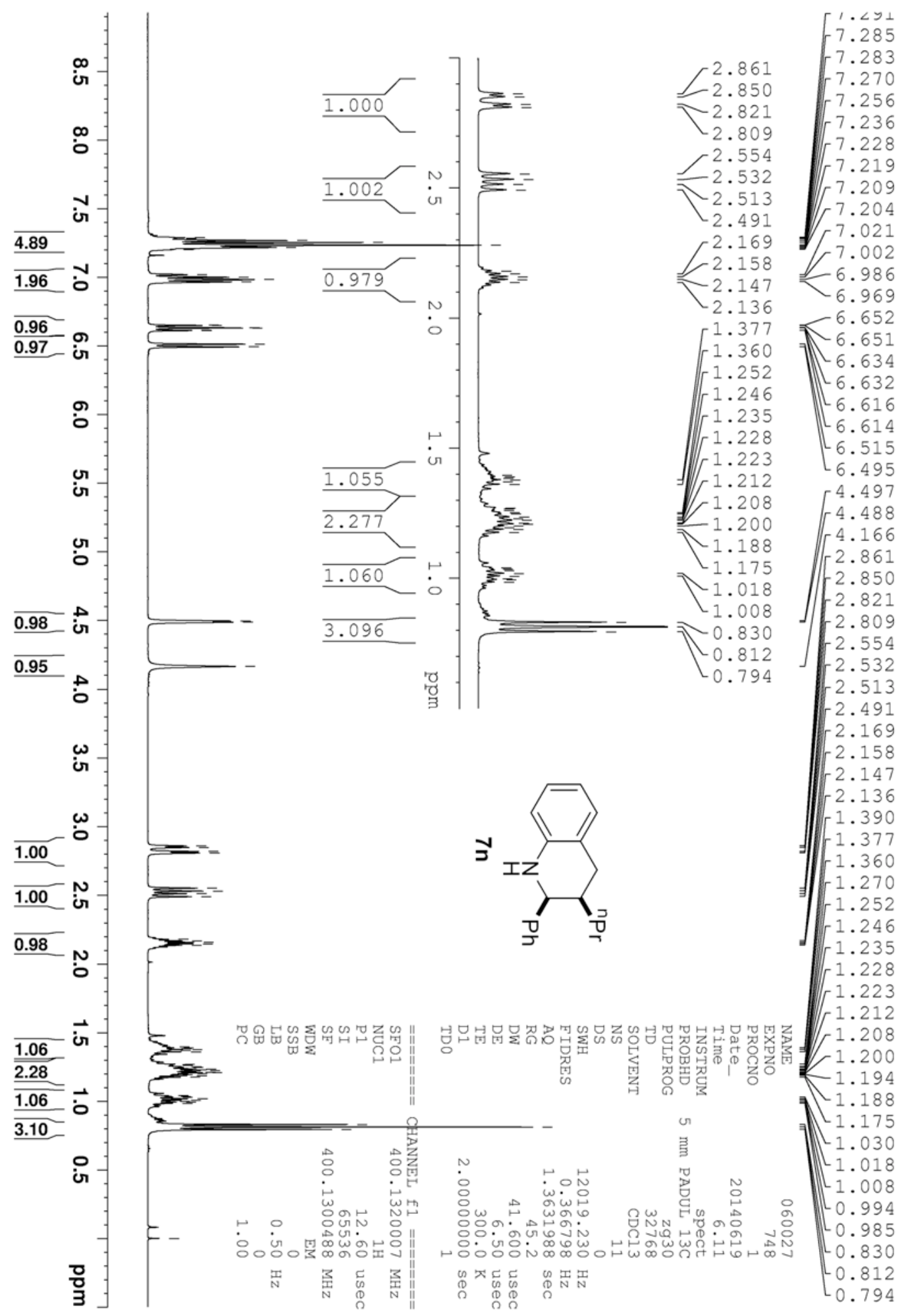




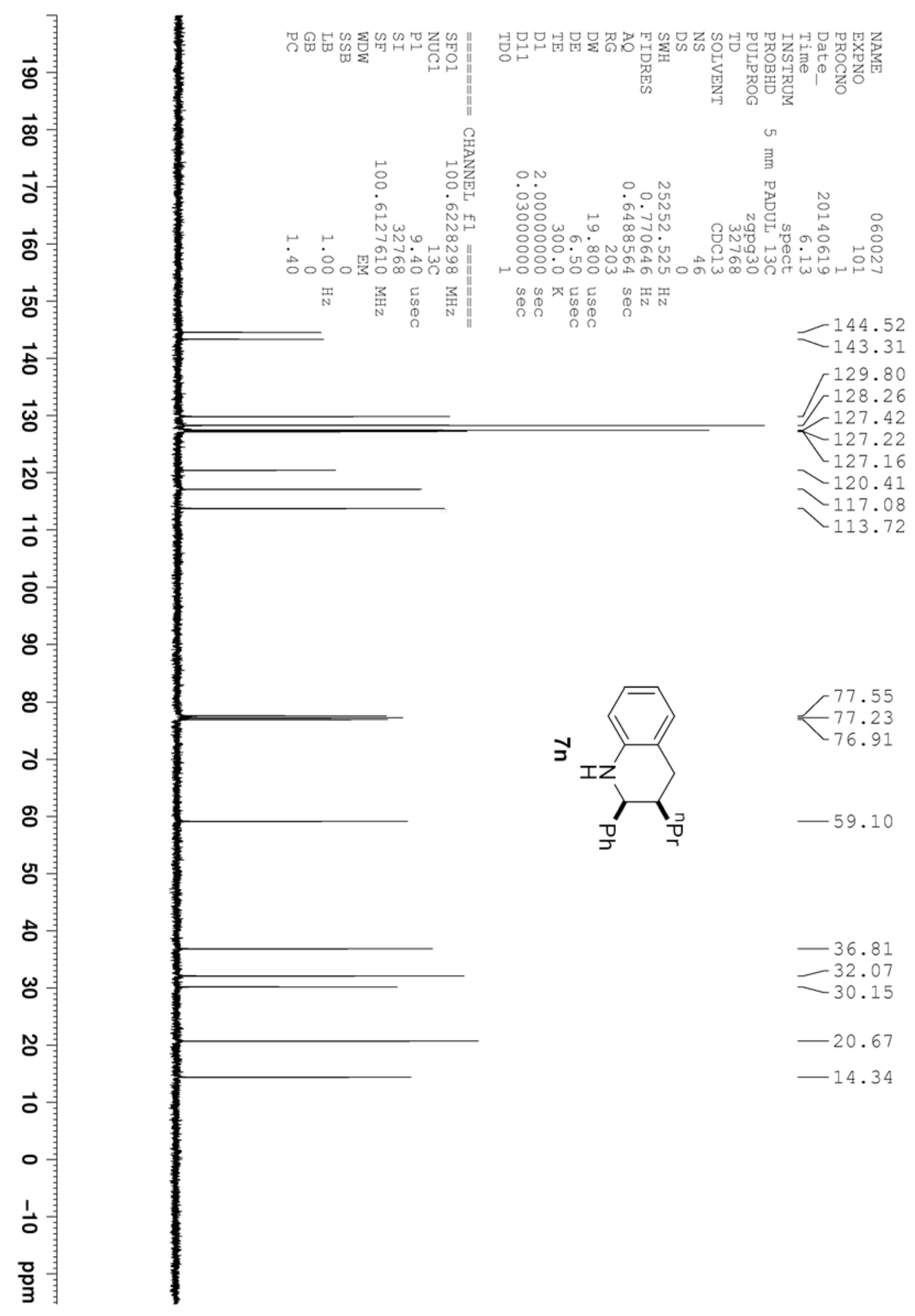




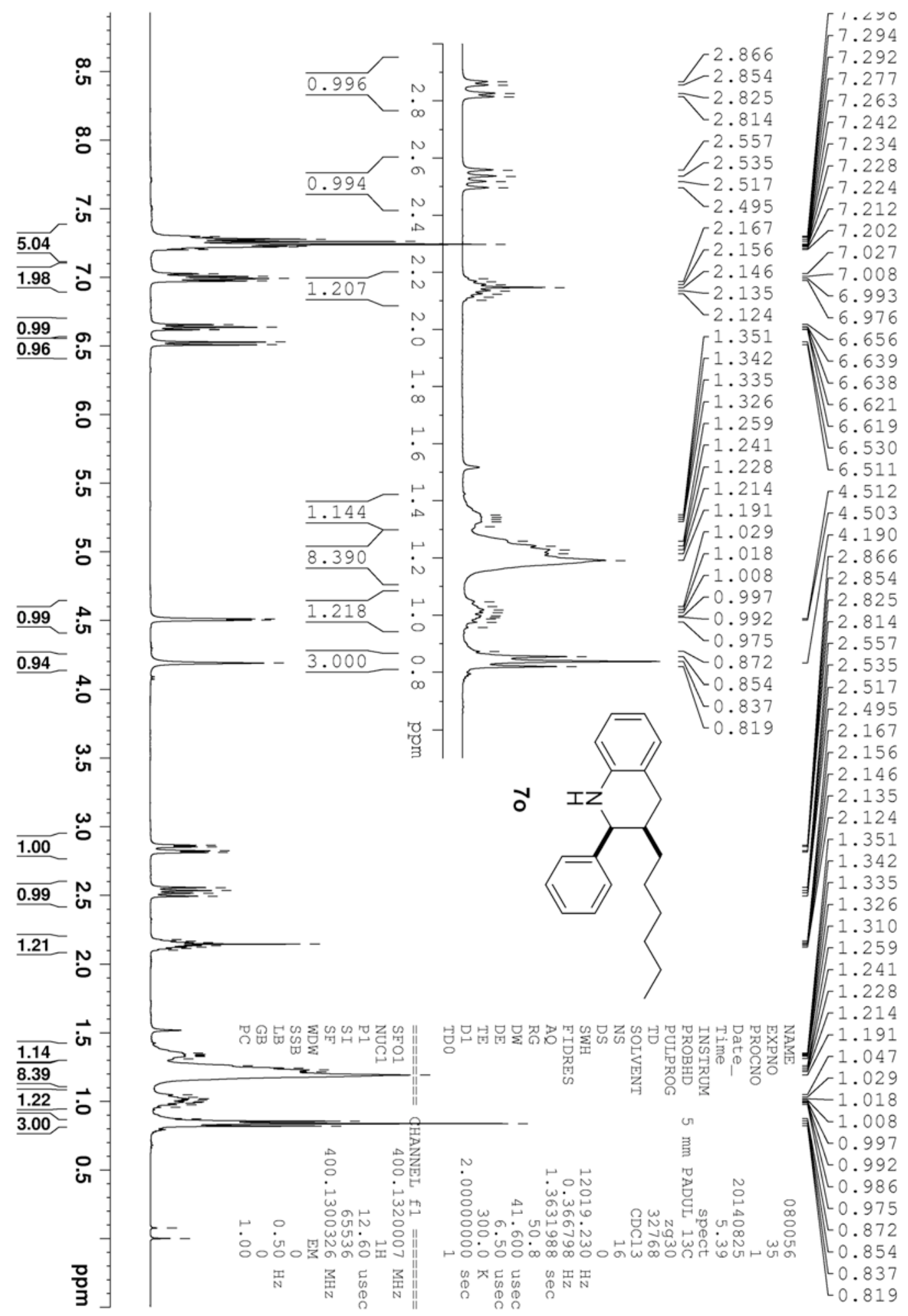




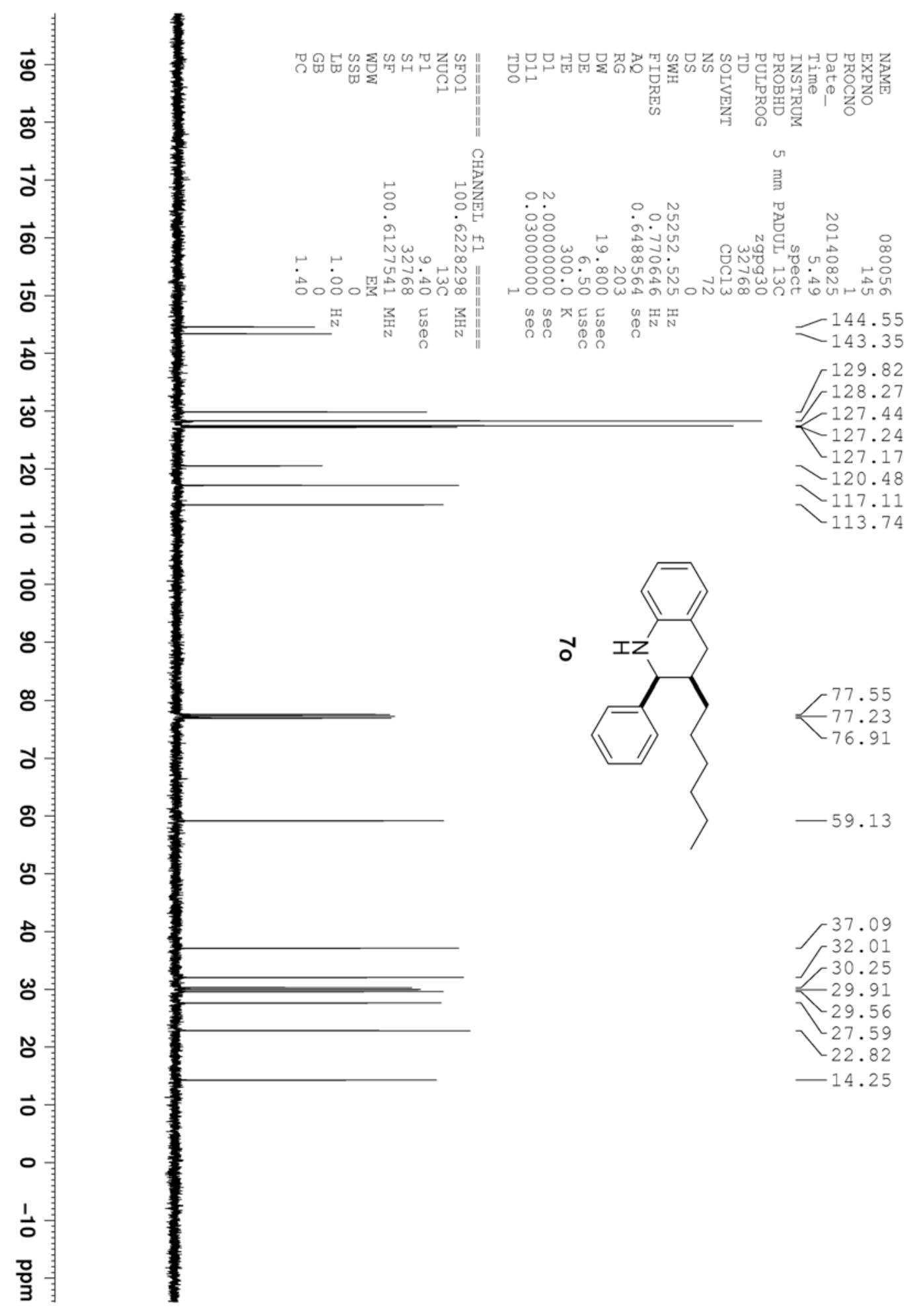

